UWThPh-1998-61

DFTT 69/98

KIAS-P98045

SFB 375-310

TUM-HEP 340/98

hep-ph/9812360

\title{
Phenomenology of Neutrino Oscillations
}

\author{
S.M. Bilenky \\ Joint Institute for Nuclear Research, Dubna, Russia, and \\ Institut für Theoretische Physik, Technische Universität München, D-85748 Garching, \\ Germany \\ C. Giunti \\ INFN, Sezione di Torino, and Dipartimento di Fisica Teorica, \\ Università di Torino, Via P. Giuria 1, I-10125 Torino, Italy, and \\ School of Physics, Korea Institute for Advanced Study, Seoul 130-012, Korea \\ W. Grimus \\ Institute for Theoretical Physics, University of Vienna, \\ Boltzmanngasse 5, A-1090 Vienna, Austria
}

\begin{abstract}
This review is focused on neutrino mixing and neutrino oscillations in the light of the recent experimental developments. After discussing possible types of neutrino mixing for Dirac and Majorana neutrinos and considering in detail the phenomenology of neutrino oscillations in vacuum and matter, we review all existing evidence and indications in favour of neutrino oscillations that have been obtained in the atmospheric, solar and LSND experiments. We present the results of the analyses of the neutrino oscillation data in the framework of mixing of three and four massive neutrinos and investigate possibilities to test the different neutrino mass and mixing schemes obtained in this way. We also discuss briefly future neutrino oscillation experiments.
\end{abstract}




\section{Contents}

\begin{tabular}{lll}
\hline 1 & Introduction & 3
\end{tabular}

2 Neutrino Mixing $\quad 5$

2.1 Dirac mass term. . . . . . . . . . . . . . . . . . . . . . 7

2.2 Dirac-Majorana mass term . . . . . . . . . . . . . . . . . . . . . 8

2.3 Majorana mass term . . . . . . . . . . . . . . . . . . . . . . . . . 12

2.4 The one-generation case . . . . . . . . . . . . . . . . . . . . . . . . . . . . . . . . . . . . . . . . . . . . .

2.5 The see-saw mechanism . . . . . . . . . . . . . . . . . . . . . . . . . . . . . . . . . . . . . . . . . . . .

2.6 Effective Lagrangians . . . . . . . . . . . . . . . . . . . . . . . . . . . . . 16

2.7 Maximal mixing . . . . . . . . . . . . . . . . . . . . . . . . 17

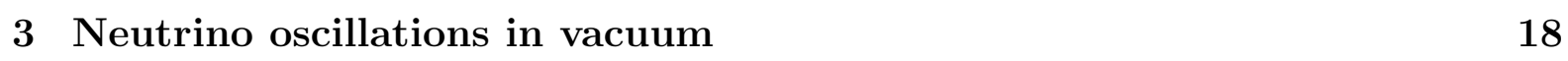

3.1 The general formalism . . . . . . . . . . . . . . . . . . . . . . . . . . . 18

3.2 Oscillations in the two-neutrino case . . . . . . . . . . . . . . . . 22

4 Neutrino oscillations and transitions in matter $\quad 26$

4.1 The effective Hamiltonian for neutrinos in matter . . . . . . . . . . . . . 26

4.2 The two-neutrino case and adiabatic transitions . . . . . . . . . . . . . . 30

4.3 The resonance . . . . . . . . . . . . . . . . . . . 33

4.4 Non-adiabatic neutrino oscillations in matter and crossing probabilities . 34

$\begin{array}{lll}5 & \text { Indications of neutrino oscillations } & 37\end{array}$

5.1 Atmospheric neutrino experiments . . . . . . . . . . . . . . . . . . 37

5.1 .1 The atmospheric neutrino flux . . . . . . . . . . . . . . . . . . . 38

5.1 .2 Experiments with atmospheric neutrinos . . . . . . . . . . . . . . 42

$5.1 .3 \quad$ The atmospheric neutrino anomaly . . . . . . . . . . . . . . . . . 44

5.1 .4 Long-baseline experiments and tests of the atmospheric neutring oscillation parameters ................ 49

5.2 Solar neutrino experiments . . . . . . . . . . . . . . . . . . . . . . . . 50

5.3 The LSND experiment . . . . . . . . . . . . . . . . . . . . . . . 63

\begin{tabular}{|lll}
6 & Analysis of neutrino oscillation data & 66
\end{tabular}

6.1 Mixing of three massive neutrinos . . . . . . . . . . . . . . . . . . . 67

6.1.1 Solar neutrinos . . . . . . . . . . . . . . . . . . . 69

6.1 .2 Atmospheric neutrinos . . . . . . . . . . . . . . . . . 70

$6.1 .3 \quad$ The mixing matrix in the case $\left|U_{e 3}\right| \ll 1$. . . . . . . . . . . . . . 72

6.1.4 Accelerator long-baseline experiments . . . . . . . . . . . . . . . . 74

6.2 Mixing of four massive neutrinos f . . . . . . . . . . . . . . . . . . 75

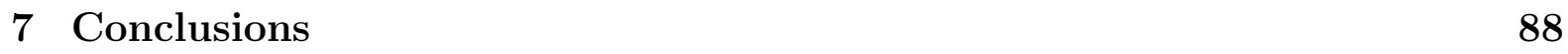

\begin{tabular}{|ll}
\hline A Properties of Majorana neutrinos and fields & 92
\end{tabular} 


\section{Introduction}

The strong evidence in favour of oscillations of atmospheric neutrinos found by the SuperKamiokande Collaboration [1, 2] opened a new era in particle physics. There is no doubt that new experiments are necessary to understand the nature of neutrino masses and mixing which are intimately connected with neutrino oscillations, but the first decisive step has been done: massive and mixed neutrinos can now be considered as real physical objects.

The problem of neutrino mass has a long history. Originally, Pauli considered the neutrino as a particle with a small but non-zero mass (smaller than the electron mass) [3] and the method for the measurement of the neutrino mass through the investigation of the $\beta$-spectrum near the end point was proposed in the first theoretical papers on $\beta$-decay of Fermi [4, [0] and Perrin [6].

The first experiments on the measurement of the neutrino mass, based on the FermiPerrin method, yielded the upper bound $m_{\nu} \lesssim 500 \mathrm{eV}$ [7] which was improved in the fifties to $m_{\nu} \lesssim 250 \mathrm{eV}$ [8]. Therefore, it became evident that the neutrino mass (if non-zero at all) is much smaller than the electron mass. This was the main reason that in 1957, after the discovery of parity violation in $\beta$-decay, the authors of the two-component theory of the neutrino (Landau [9], Lee and Yang [10], Salam [11]) assumed that the neutrino is a massless particle, the field of which is either a left-handed field $\nu_{L}$ or a right-handed field $\nu_{R}$.

In 1958, Goldhaber et al. [12 measured the helicity of the neutrino. The result of this experiment was in agreement with the two-component neutrino theory and it was established that the neutrino field is $\nu_{L}$. 1 The results of the experiment of Goldhaber et al. could not exclude, however, the possibility of a small neutrino mass. In the $\mathrm{V}-\mathrm{A}$ theory (Feynman and Gell-Mann [15], Sudarshan and Marshak [16]) the Hamiltonian of weak interactions contains the left-handed component of the neutrino field $\nu_{L}$, and also the left-handed components of all massive fields. Therefore the possibility for the neutrino to be nevertheless a massive particle became more natural [17] after the confirmation of the $\mathrm{V}-\mathrm{A}$ theory.

In 1957, B. Pontecorvo [18,19] proposed the idea that the state of neutrinos produced in weak interaction processes is a superposition of states of two Majorana neutrinos [20] with definite masses (analogous to the states $\left|K^{0}\right\rangle$ and $\left|\bar{K}^{0}\right\rangle$ which are the superposition of $\left|K_{1}\right\rangle$ and $\left|K_{2}\right\rangle$, the states of particles with definite masses and widths). In this way, B. Pontecorvo arrived at the hypothesis of neutrino oscillations (analogous to $K^{0} \leftrightarrows \bar{K}^{0}$ oscillations). At that time only one type of neutrino was known. The possibility of mixing of the two species of neutrinos $\nu_{e}$ and $\nu_{\mu}$ was considered in Ref. [21]. All possible types of neutrino oscillations for this case were investigated by Pontecorvo in 1967 [22].

Gribov and Pontecorvo proposed in 1969 [23] the first phenomenological theory of neutrino mixing and oscillations. In this theory, the two left-handed neutrino fields $\nu_{e L}$

\footnotetext{
${ }^{1}$ Notice that in the Goldhaber et al. experiment the helicity of the electron neutrino was measured. The helicity of the muon neutrino was measured in several experiments (for the references see the review of V.L. Telegdi [13]). The best accuracy in the measurement of the muon neutrino was achieved in the experiment by Grénacs et al. [14].
} 
and $\nu_{\mu L}$ are linear combinations of the left-handed components of the fields of Majorana neutrinos with definite masses and the neutrino mass term contains only the left-handed fields $\nu_{e L}$ and $\nu_{\mu L}$.

In 1976, neutrino oscillations were considered in the scheme of mixing of two Dirac neutrinos based on the analogy between quarks and leptons [24, 25] and in the same year in the general Dirac-Majorana scheme [26] (for later works see Refs. [27 30]).

The theoretical arguments in favour of non-zero neutrino masses and mixing are based on the models beyond the Standard Model (see, for example, Ref. [31]). In such models the fields of quarks, charged leptons and neutrinos are grouped in the same multiplets and the generation of the masses of quarks and charged leptons with the Higgs mechanism as a rule provides also non-zero neutrino masses.

In 1979, the see-saw mechanism for the generation of neutrino masses was proposed 32 34. This mechanism connects the smallness of neutrino masses with the possible violation of lepton number conservation at a very large energy scale.

At present the effects of neutrino masses and mixing are investigated in many different experiments. There are three types of experiments in which the effects of small neutrino masses (say, of the order of $1 \mathrm{eV}$ or smaller) and mixing can be revealed (for a review and references see Ref. [35]):

1. Neutrino oscillations experiments.

2. Experiments on the search for neutrinoless double $\beta$-decay.

3. Experiments on the measurement of the electron neutrino mass with the precise investigation of the high energy part of the $\beta$-spectrum of ${ }^{3} \mathrm{H}$.

Three indications in favour of neutrino masses and mixing have been found so far. These indications were obtained in the following experiments:

1. Solar neutrino experiments (Homestake [36 38], Kamiokande [39 41], GALLEX 42, 43, SAGE 44, 45], Super-Kamiokande 46 48]).

2. Atmospheric neutrino experiments (Super-Kamiokande [1, 2], Kamiokande [49], IMB [50], Soudan [51], MACRO [52]).

3. The accelerator LSND experiment [53,54].

Many other neutrino oscillation experiments with neutrinos from reactor and accelerators did not find any evidence for neutrino oscillations. In the experiments on the search for neutrinoless double $\beta$-decay no indications for non-zero neutrino masses were found (see Section 6.2). The present upper bound for the electron neutrino mass obtained in the Troitsk experiment [55] is $2.7 \mathrm{eV}$ (see also the Mainz experiment [56]). The upper limits on the masses of $\nu_{\mu}$ and $\nu_{\tau}$ are $170 \mathrm{keV}$ (90\% CL) and $18.2 \mathrm{MeV}$ (95\% CL), respectively [35. Neutrinos play an important role in cosmology and astrophysics and many bounds on neutrino properties can be derived in this context. For reviews see, e.g., Refs. [31, 57. 
In this review we discuss the phenomenological theory of neutrino mixing (Section 2), neutrino oscillations in vacuum (Section 3), neutrino oscillations and transitions in matter (Section 4) and experimental data and results of analyses of the data (Sections 5 and 6). We also consider the implications of the existing experimental results on neutrino oscillations for experiments in preparation. After the conclusions (Section 7) we discuss some properties of Majorana neutrinos and fields in Appendix A.

We hope that this review will be useful not only for the physicists that are working in the field but also for those who are interested in this exciting field of physics. In many cases we present not only results but also derivations of the results. For those who start to study the subject we refer to the books Refs. [31,58 61] and the reviews Refs. 62 63].

\section{Neutrino Mixing}

All the numerous data on weak interaction processes are perfectly well described by the Standard Model [74 76. The standard weak interactions are due to the coupling of quarks and leptons with the gauge $W$ and $Z$ vector bosons, described by the charged-current (CC) and neutral-current (NC) interaction Lagrangians

$$
\begin{aligned}
\mathcal{L}_{I}^{\mathrm{CC}} & =-\frac{g}{2 \sqrt{2}} j_{\rho}^{\mathrm{CC}} W^{\rho}+\text { h.c. }, \\
\mathcal{L}_{I}^{\mathrm{NC}} & =-\frac{g}{2 \cos \theta_{W}} j_{\rho}^{\mathrm{NC}} Z^{\rho} .
\end{aligned}
$$

Here $g$ is the $\mathrm{SU}(2)_{L}$ gauge coupling constant, $\theta_{W}$ is the weak angle and the charged and neutral currents $j_{\rho}^{\mathrm{CC}}$ and $j_{\rho}^{\mathrm{NC}}$ are given by the expressions

$$
\begin{aligned}
& j_{\rho}^{\mathrm{CC}}=2 \sum_{\ell=e, \mu, \tau} \overline{\nu_{\ell L}} \gamma_{\rho} \ell_{L}+\ldots \\
& j_{\rho}^{\mathrm{NC}}=\sum_{\ell=e, \mu, \tau} \overline{\nu_{\ell L}} \gamma_{\rho} \nu_{\ell L}+\ldots
\end{aligned}
$$

where the $\ell$ are the physical charged lepton fields with masses $m_{\ell}$ and we have written explicitly only the terms containing the neutrino fields. The flavour neutrinos $\nu_{e}, \nu_{\mu}, \ldots$ are determined by $\mathrm{CC}$ weak interactions: for example, the $\nu_{\mu}$ is the particle produced in the decay $\pi^{+} \rightarrow \mu^{+}+\nu_{\mu}$ and so on. The number of light flavour neutrinos is given by the invisible width of the $Z$ boson [77 in the Standard Model. It was shown in the famous LEP experiments on the measurement of the invisible width of the $Z$ boson that the number of the light flavour neutrinos is equal to 3 . The most recent experimental value of the number of neutrino flavours is $2.994 \pm 0.012$ [35], showing that are no other neutrino flavours than the well-known $\nu_{e}, \nu_{\mu}, \nu_{\tau}$.

The CC and NC interactions conserve the electron $L_{e}$, muon $L_{\mu}$ and tau $L_{\tau}$ lepton numbers, which are assigned as shown in Table 2.1.

There are no indications in favour of violation of the law of conservation of lepton numbers in weak processes and very strong bounds on the probabilities of the lepton 


\begin{tabular}{|cccc|}
\hline & $L_{e}$ & $L_{\mu}$ & $L_{\tau}$ \\
\hline$\left(\nu_{e}, e^{-}\right)$ & +1 & 0 & 0 \\
$\left(\nu_{\mu}, \mu^{-}\right)$ & 0 & +1 & 0 \\
$\left(\nu_{\tau}, \tau^{-}\right)$ & 0 & 0 & +1 \\
\hline
\end{tabular}

Table 2.1. Assignment of lepton numbers. The corresponding antiparticles have opposite lepton numbers.

number violating processes have been obtained from the experimental data. The most stringent limits (90\% CL) are (see Ref. [35]):

$$
\begin{aligned}
& \Gamma(\mu \rightarrow e \gamma) / \Gamma(\mu \rightarrow \text { all })<4.9 \times 10^{-11}, \\
& \Gamma(\mu \rightarrow 3 e) / \Gamma(\mu \rightarrow \text { all })<1.0 \times 10^{-12}, \\
& \sigma\left(\mu^{-} \mathrm{Ti} \rightarrow e^{-} \mathrm{Ti}\right) / \sigma\left(\mu^{-} \mathrm{Ti} \rightarrow \text { capture }\right)<4.3 \times 10^{-12}, \\
& \Gamma\left(K_{L} \rightarrow e \mu\right) / \Gamma\left(K_{L} \rightarrow \text { all }\right)<3.3 \times 10^{-11}, \\
& \Gamma\left(K^{+} \rightarrow \pi^{+} e^{-} \mu^{+}\right) / \Gamma\left(K^{+} \rightarrow \text { all }\right)<2.1 \times 10^{-10} .
\end{aligned}
$$

According to the neutrino mixing hypothesis [18, 19,21], the conservation of the lepton numbers is only approximate. It is violated because of non-zero neutrino masses and neutrino mixing. In the case of neutrino mixing, the left-handed flavour neutrino fields $\nu_{\ell L}$ are superpositions of the left-handed components $\nu_{k L}$ of the fields of neutrinos with definite masses $m_{k}$ :

$$
\nu_{\ell L}=\sum_{k=1}^{n} U_{\ell k} \nu_{k L} \quad(\ell=e, \mu, \tau),
$$

where $U$ is a unitary mixing matrix. The number of massive neutrino fields $n$ could be equal or more than 3 . If $n$ is larger than 3 there are sterile neutrinos that do not take part in the standard weak interactions (2.3) and (2.4).

It is well-established that quarks take part in $\mathrm{CC}$ weak interactions in mixed form with the $\mathrm{V}-\mathrm{A}$ current

$$
\sum_{q^{\prime}=u, c, t} \sum_{q=d, s, b} \bar{q}_{L}^{\prime} \gamma_{\rho} V_{q^{\prime} q} q_{L}
$$

where $V$ is the Cabibbo-Kobayashi-Maskawa mixing matrix [78, 79]. The relation (2.10) is analogous to the mixing in Eq.(2.11). However, between the mixing of neutrinos and quarks there can be a fundamental difference. In fact, quarks are four-component Dirac particles: quarks and antiquarks have opposite electric and baryonic charges. Instead, neutrinos are electrically neutral particles. If the total lepton charge

$$
L=L_{e}+L_{\mu}+L_{\tau}
$$

is conserved, neutrinos with definite masses are four-component Dirac particles like quarks (in this case a neutrino differs from an antineutrino by the opposite value of $L$ ). If the total lepton number (2.12) is not conserved, massive neutrinos are truly neutral twocomponent Majorana particles. These possibilities are realized in different models and correspond to different neutrino mass terms. 


\subsection{Dirac mass term}

A Dirac neutrino mass term can be generated by the Higgs mechanism with the standard Higgs doublet which is responsible for the generation of the masses of quarks and charged leptons. In this case the neutrino mass term is given by

$$
\mathcal{L}^{\mathrm{D}}=-\sum_{\ell, \ell^{\prime}} \overline{\nu_{\ell R}} M_{\ell \ell^{\prime}}^{\mathrm{D}} \nu_{\ell^{\prime} L}+\text { h.c. } \quad(\ell=e, \mu, \tau)
$$

and $M^{\mathrm{D}}$ is a complex $3 \times 3$ matrix.

The Dirac mass term (2.13) can be diagonalized taking into account that the complex matrix $M^{\mathrm{D}}$ can be written as

$$
M^{\mathrm{D}}=V \hat{m} U^{\dagger}
$$

where $V$ and $U$ are unitary matrices and $\hat{m}$ is a positive definite diagonal matrix, $\hat{m}_{k j}=$ $m_{k} \delta_{k j}$, with $m_{k} \geq 0$. Hence, the Dirac mass term (2.13) can be written in the diagonal form

$$
\mathcal{L}^{\mathrm{D}}=-\sum_{k=1}^{3} m_{k} \overline{\nu_{k}} \nu_{k}
$$

with

$$
\nu_{\ell L}=\sum_{k=1}^{3} U_{\ell k} \nu_{k L} \quad(\ell=e, \mu, \tau) .
$$

Therefore, in the case of the Dirac mass term (2.13) the three flavour fields $\nu_{\ell L}(\ell=$ $e, \mu, \tau)$ are linear unitary combinations of the left-handed components $\nu_{k L}$ of three fields of neutrinos with masses $m_{k}(k=1,2,3)$. On the other hand, we also have

$$
\nu_{\ell R}=\sum_{k=1}^{3} V_{\ell k} \nu_{k R} \quad(\ell=e, \mu, \tau),
$$

but these right-handed fields do not occur in the standard weak interaction Lagrangian. Therefore, right-handed singlets are sterile and are not mixed in this scheme with the active neutrinos. . $^{2}$

The field $\nu_{k}$ is a Dirac field if not only the mass term (2.13) but also the total Lagrangian is invariant under the global $\mathrm{U}(1)$ transformation

$$
\nu_{\ell} \rightarrow e^{i \varphi} \nu_{\ell}, \quad \ell \rightarrow e^{i \varphi} \ell \quad(\ell=e, \mu, \tau),
$$

\footnotetext{
${ }^{2}$ One needs to assume the existence of right-handed $\mathrm{SU}(2)_{L}$ singlet fields $\nu_{\ell R}$. If these fields do not exist, it is not possible to construct a Dirac mass term for the neutrinos, i.e., neutrinos are massless particles. This model is sometimes called Minimal Standard Model [74 76].

${ }^{3}$ Note that in this review we use four-component spinors. For the equivalent alternative of twocomponent spinors see, e.g., Refs. [30, 80].

${ }^{4}$ In the Dirac case it is possible to conceive also the existence of left-handed sterile singlet fields mixed with the active flavour neutrino fields, in contrast to the right-handed singlets which cannot be mixed with the active neutrinos because of lepton number conservation.
} 
where the phase $\varphi$ is the same for all neutrino and charged lepton fields. Then, using Noether's theorem, one can see that the invariance of the Lagrangian under the transformation (2.18) implies that the total lepton charge $L$ is conserved. Therefore, $L$ is the quantum number that distinguishes a neutrino from an antineutrino.

The Dirac mass term (2.13) allows processes like $\mu \rightarrow e+\gamma, \mu^{-} \rightarrow e^{-}+e^{+}+e^{-}$. However, the contribution of neutrino mixing to the probabilities of such processes is negligibly small 81 83.

The unitary $3 \times 3$ mixing matrix $U$ can be written in terms of 3 mixing angles and 6 phases. However, only one phase is measurable [79]. This is due to the fact that in the Standard Model the only term in the Lagrangian where the mixing matrix $U$ enters is the CC interaction Lagrangian (2.1). With neutrino mixing the lepton charged-current is given by

$$
j_{\rho}^{\mathrm{CC} \dagger}=2 \sum_{\ell=e, \mu, \tau} \overline{\ell_{L}} \gamma_{\rho} \nu_{\ell L}=2 \sum_{\ell=e, \mu, \tau} \sum_{k=1}^{3} \overline{\ell_{L}} \gamma_{\rho} U_{\ell k} \nu_{k L}
$$

Because the phases of Dirac fields are arbitrary one can eliminate from the mixing matrix $U$ five phases by a redefinition of the phases of the charged lepton and neutrino fields, with only one physical phase remaining in $U$. The presence of this phase causes the violation of $\mathrm{CP}$ invariance in the lepton sector. A convenient parameterization of the mixing matrix $U$ is the one proposed in Ref. [84]:

$$
U=\left(\begin{array}{ccc}
c_{12} c_{13} & s_{12} c_{13} & s_{13} e^{i \delta_{13}} \\
-s_{12} c_{23}-c_{12} s_{23} s_{13} e^{i \delta_{13}} & c_{12} c_{23}-s_{12} s_{23} s_{13} e^{i \delta_{13}} & s_{23} c_{13} \\
s_{12} s_{23}-c_{12} c_{23} s_{13} e^{i \delta_{13}} & -c_{12} s_{23}-s_{12} c_{23} s_{13} e^{i \delta_{13}} & c_{23} c_{13}
\end{array}\right)
$$

where $c_{i j} \equiv \cos \vartheta_{i j}$ and $s_{i j} \equiv \sin \vartheta_{i j}$ and $\delta_{13}$ is the CP-violating phase.

Since in the parameterization (2.20) of the mixing matrix the CP-violating phase $\delta_{13}$ is associated with $s_{13}$, it is clear that $\mathrm{CP}$ violation is negligible in the lepton sector if the mixing angle $\vartheta_{13}$ is small. More generally, it is possible to show that if any of the elements of the mixing matrix is zero, the CP-violating phase can be rotated away by a suitable rephasing of the charged lepton and neutrino fields. ${ }^{5}$

\subsection{Dirac-Majorana mass term}

If none of the lepton numbers is conserved and both, left-handed flavour fields $\nu_{\ell L}(\ell=$ $e, \mu, \tau)$ and sterile right-handed gauge singlet fields $\nu_{s R}\left(s=s_{1}, s_{2}, \ldots\right)$ enter into the mass term we have the so-called Dirac-Majorana mass term

$$
\mathcal{L}^{\mathrm{D}+\mathrm{M}}=\mathcal{L}_{L}^{\mathrm{M}}+\mathcal{L}^{\mathrm{D}}+\mathcal{L}_{R}^{\mathrm{M}},
$$

with

$$
\mathcal{L}^{\mathrm{D}}=-\sum_{s, \ell} \overline{\nu_{s R}} M_{s \ell}^{\mathrm{D}} \nu_{\ell L}+\text { h.c. },
$$

\footnotetext{
${ }^{5}$ This can also be seen by noticing that the Jarlskog rephasing-invariant parameter 85 87 vanishes if one of the elements of the mixing matrix is zero.
} 


$$
\begin{aligned}
\mathcal{L}_{L}^{\mathrm{M}} & =-\frac{1}{2} \sum_{\ell, \ell^{\prime}} \overline{\left(\nu_{\ell L}\right)^{c}} M_{\ell \ell^{\prime}}^{L} \nu_{\ell^{\prime} L}+\text { h.c. }, \\
\mathcal{L}_{R}^{\mathrm{M}} & =-\frac{1}{2} \sum_{s, s^{\prime}} \overline{\nu_{s R}} M_{s s^{\prime}}^{R}\left(\nu_{s^{\prime} R}\right)^{c}+\text { h.c. }
\end{aligned}
$$

Here $M^{\mathrm{D}}, M^{L}$ and $M^{R}$ are complex matrices and the indices $s, s^{\prime}$ run over $n_{R}$ values. Let us stress that the number of right-handed singlet fields could be different from the number of neutrino flavours (see [30, 88]).

The charge-conjugate fields are defined by

$$
\left(\nu_{\ell L}\right)^{c} \equiv \mathcal{C}{\overline{\nu_{\ell L}}}^{T}, \quad\left(\nu_{s R}\right)^{c} \equiv \mathcal{C}{\overline{\nu_{s R}}}^{T}
$$

where $\mathcal{C}$ is the charge conjugation matrix (see Appendix A). Notice that $\left(\nu_{\ell L}\right)^{c}$ and $\left(\nu_{s R}\right)^{c}$ are right-handed and left-handed components, respectively. Indeed, with $\mathcal{C}^{-1} \gamma_{5} \mathcal{C}=\gamma_{5}^{T}$ one finds

$$
\frac{1+\gamma_{5}}{2}\left(\nu_{\ell L}\right)^{c}=\mathcal{C}\left(\overline{\nu_{\ell L}} \frac{1+\gamma_{5}}{2}\right)^{T}=\mathcal{C}{\overline{\nu_{\ell L}}}^{T}=\left(\nu_{\ell L}\right)^{c}
$$

and the analogous reasoning holds for $\left(\nu_{s R}\right)^{c}$. Furthermore, we have

$$
\overline{\left(\nu_{\ell L}\right)^{c}}=-\nu_{\ell L}^{T} \mathcal{C}^{-1}, \quad \overline{\left(\nu_{s R}\right)^{c}}=-\nu_{s R}^{T} \mathcal{C}^{-1} .
$$

The matrices $M^{L}$ and $M^{R}$ are symmetric. This can be shown with the help of the relation $\nu_{\ell L}^{T} \mathcal{C}^{-1} \nu_{\ell^{\prime} L}=\nu_{\ell^{\prime} L}^{T} \mathcal{C}^{-1} \nu_{\ell L}$, which follows from the fact that $\mathcal{C}$ is an antisymmetric matrix and from the anticommutation property of fermion fields. An analogous relation holds for the right-handed fields. Then, using Eq.(2.27), one obtains

$$
\sum_{\ell, \ell^{\prime}} \overline{\left(\nu_{\ell L}\right)^{c}} M_{\ell \ell^{\prime}}^{L} \nu_{\ell^{\prime} L}=-\sum_{\ell, \ell^{\prime}} \nu_{\ell^{\prime} L}^{T} \mathcal{C}^{-1} M_{\ell \ell^{\prime}}^{L} \nu_{\ell L}=\sum_{\ell, \ell^{\prime}} \overline{\left(\nu_{\ell L}\right)^{c}} M_{\ell^{\prime} \ell}^{L} \nu_{\ell^{\prime} L} .
$$

Let us introduce the left-handed column vector

$$
n_{L} \equiv\left(\begin{array}{c}
\nu_{L} \\
\left(\nu_{R}\right)^{c}
\end{array}\right) \quad \text { with } \quad \nu_{L} \equiv\left(\begin{array}{c}
\nu_{e L} \\
\nu_{\mu L} \\
\nu_{\tau L}
\end{array}\right)
$$

where $\nu_{R}$ is the column vector of right-handed fields. With the relation

$$
\sum_{s, \ell} \overline{\nu_{s R}} M_{s \ell}^{\mathrm{D}} \nu_{\ell L}=-\sum_{s, \ell} \nu_{\ell L}^{T} M_{s \ell}^{\mathrm{D}}{\overline{\nu_{s R}}}^{T}=\sum_{s, \ell} \overline{\left(\nu_{\ell L}\right)^{c}}\left(M^{\mathrm{D}}\right)_{\ell s}^{T}\left(\nu_{s R}\right)^{c}
$$

the Dirac-Majorana mass term $\mathcal{L}^{\mathrm{D}+\mathrm{M}}$ can be written in the form

$$
\mathcal{L}^{\mathrm{D}+\mathrm{M}}=-\frac{1}{2} \overline{\left(n_{L}\right)^{c}} M^{\mathrm{D}+\mathrm{M}} n_{L}+\text { h.c. },
$$

with the symmetric $\left(3+n_{R}\right) \times\left(3+n_{R}\right)$ matrix

$$
M^{\mathrm{D}+\mathrm{M}} \equiv\left(\begin{array}{cc}
M^{L} & \left(M^{\mathrm{D}}\right)^{T} \\
M^{\mathrm{D}} & M^{R}
\end{array}\right)
$$


The complex symmetric matrix $M^{\mathrm{D}+\mathrm{M}}$ can be diagonalized with the help of a unitary matrix $U$ [89, 90]:

$$
M^{\mathrm{D}+\mathrm{M}}=\left(U^{\dagger}\right)^{T} \hat{m} U^{\dagger},
$$

with $\hat{m}_{k j}=m_{k} \delta_{k j}$ and $m_{k} \geq 0$. Using this relation the mass term (2.31) can be written as

$$
\mathcal{L}^{\mathrm{D}+\mathrm{M}}=-\frac{1}{2} \bar{N} \hat{m} N=-\frac{1}{2} \sum_{k=1}^{3+n_{R}} m_{k} \overline{\nu_{k}} \nu_{k},
$$

with

$$
N \equiv\left(\begin{array}{c}
\nu_{1} \\
\nu_{2} \\
\vdots
\end{array}\right)=U^{\dagger} n_{L}+\left(U^{\dagger} n_{L}\right)^{c} .
$$

The fields $\nu_{k}$ satisfy the Majorana condition

$$
\left(\nu_{k}\right)^{c}=\nu_{k} \quad\left(k=1, \ldots, 3+n_{R}\right) .
$$

Therefore, in the general case of a Dirac-Majorana mass term the fields of particles with definite masses are Majorana fields. Majorana particles are particles with spin 1/2 and all charges equal to zero (particle $=$ antiparticle). Some properties of Majorana fields are discussed in Appendix A.

We want to emphasize that it is natural that the diagonalization of the mass term (2.31) leads to fields of Majorana particles with definite masses: the mass term (2.31) for the case of a general matrix $M^{\mathrm{D}+\mathrm{M}}$ is not invariant under any global phase transformation. In other words, in the general case of the Dirac and Majorana mass term there are no conserved quantum numbers that allow to distinguish a particle from its antiparticle.

From Eq.(2.35), for the left-handed components of the neutrino fields we have the mixing relations

$$
\begin{aligned}
& \nu_{\ell L}=\sum_{k=1}^{3+n_{R}} U_{\ell k} \nu_{k L} \quad(\ell=e, \mu, \tau), \\
& \left(\nu_{s R}\right)^{c}=\sum_{k=1}^{3+n_{R}} U_{s k} \nu_{k L} .
\end{aligned}
$$

Thus, in the case of a Dirac-Majorana mass term the flavour fields $\nu_{\ell L}$ are linear unitary combinations of the left-handed components of the Majorana fields $\nu_{k}$ of neutrinos with definite masses. These components are also connected with the sterile fields $\left(\nu_{s R}\right)^{c}$ through the relation (2.38). If the masses $m_{k}$ are small, the mixing relations (2.37) and (2.38) imply that flavour neutrinos $\nu_{e}, \nu_{\mu}$ and $\nu_{\tau}$ can oscillate into sterile states that are quanta of the right-handed fields $\nu_{s R}$. We will discuss such transitions in Section 6 .

Let us stress that in order to have all three terms in the Dirac-Majorana mass term (2.31) not only a Higgs doublet but also a Higgs triplet 91 93 and a Higgs singlet are necessary. Thus, it can be generated only in the framework of models beyond the 
Standard Model. A typical example is the SO(10) model (see, for example, [31]). For a discussion of radiative corrections to the Dirac-Majorana mass term see Ref. [94.

Up to now we did not make any special assumption about the Dirac-Majorana mass term (2.31). Let us now consider the possibility that CP invariance in the lepton sector holds. In this case we have

$$
U_{\mathrm{CP}} \mathcal{L}^{\mathrm{D}+\mathrm{M}}(x) U_{\mathrm{CP}}^{-1}=\mathcal{L}^{\mathrm{D}+\mathrm{M}}\left(x_{\mathrm{P}}\right) .
$$

where $U_{C P}$ is the operator of CP conjugation, $x \equiv\left(x^{0}, \vec{x}\right)$ and $x_{\mathrm{P}} \equiv\left(x^{0},-\vec{x}\right)$. For the fields $n_{L}(x)$ we take the usual CP transformation

$$
U_{\mathrm{CP}} n_{L}(x) U_{\mathrm{CP}}^{-1}=\eta \gamma^{0} \mathcal{C}{\overline{n_{L}}}^{T}\left(x_{\mathrm{P}}\right),
$$

where $\eta$ is a diagonal matrix of phase factors. The mass term $\mathcal{L}^{\mathrm{D}+\mathrm{M}}$ can be written in the form

$$
\mathcal{L}^{\mathrm{D}+\mathrm{M}}(x)=\frac{1}{2} n_{L}^{T}(x) \mathcal{C}^{-1} M^{\mathrm{D}+\mathrm{M}} n_{L}(x)-\frac{1}{2} \overline{n_{L}}(x)\left(M^{\mathrm{D}+\mathrm{M}}\right)^{\dagger} \mathcal{C}{\overline{n_{L}}}^{T}(x) .
$$

Using Eq.(2.40) we obtain

$$
U_{\mathrm{CP}} n_{L}^{T}(x) \mathcal{C}^{-1} M^{\mathrm{D}+\mathrm{M}} n_{L}(x) U_{\mathrm{CP}}^{-1}=\overline{n_{L}}\left(x_{\mathrm{P}}\right) \eta M^{\mathrm{D}+\mathrm{M}} \eta \mathcal{C}{\overline{n_{L}}}^{T}\left(x_{\mathrm{P}}\right)
$$

Hence, the CP invariance condition (2.39) is satisfied if

$$
\eta M^{\mathrm{D}+\mathrm{M}} \eta=-\left(M^{\mathrm{D}+\mathrm{M}}\right)^{\dagger}
$$

Up to now $\eta$ was an arbitrary diagonal matrix of phase factors. If we choose $\eta=i$, in this case CP invariance in the lepton sector implies that

$$
M^{\mathrm{D}+\mathrm{M}}=\left(M^{\mathrm{D}+\mathrm{M}}\right)^{\dagger} .
$$

Taking into account that $M^{\mathrm{D}+\mathrm{M}}$ is a symmetric matrix, the condition (2.44) is equivalent to

$$
M^{\mathrm{D}+\mathrm{M}}=\left(M^{\mathrm{D}+\mathrm{M}}\right)^{*} .
$$

The real symmetric matrix $M^{\mathrm{D}+\mathrm{M}}$ can be diagonalized with the transformation

$$
M^{\mathrm{D}+\mathrm{M}}=\mathcal{O} m^{\prime} \mathcal{O}^{T}
$$

where $\mathcal{O}$ is an orthogonal matrix $\left(\mathcal{O}^{T}=\mathcal{O}^{-1}\right)$ and $m^{\prime}$ is a diagonal matrix, $m_{k j}^{\prime}=m_{k}^{\prime} \delta_{k j}$. The eigenvalues $m_{k}^{\prime}$ can be positive or negative and the neutrino masses are thus given by $m_{k}=\left|m_{k}^{\prime}\right|$. Therefore, we write the eigenvalues as

$$
m_{k}^{\prime}=m_{k} \rho_{k}
$$

where $\rho_{k}= \pm 1$ is the sign of the $k^{\text {th }}$ eigenvalue of the matrix $M^{\mathrm{D}+\mathrm{M}}$. Then the relation (2.46) can be rewritten in the form

$$
M^{\mathrm{D}+\mathrm{M}}=\left(U^{\dagger}\right)^{T} \hat{m} U^{\dagger}
$$


with

$$
U^{\dagger}=\sqrt{\rho} \mathcal{O}^{T}
$$

and $\hat{m}_{k j}=m_{k} \delta_{k j}$. Here $\rho$ is the diagonal matrix with elements $\rho_{k j}=\rho_{k} \delta_{k j}$. Therefore, if $\mathrm{CP}$ is conserved in the lepton sector, the neutrino mixing matrix has the simple structure shown in Eq.(2.49), which implies that

$$
U^{*}=U \rho
$$

The CP transformation of the Majorana field $\nu_{k}$ is given by (see Appendix A)

$$
U_{\mathrm{CP}} \nu_{k}(x) U_{\mathrm{CP}}^{-1}=\eta_{k}^{\mathrm{CP}} \gamma_{0} \nu_{k}\left(x_{\mathrm{P}}\right)
$$

where $\eta_{k}^{\mathrm{CP}}$ is the CP parity of the Majorana field $\nu_{k}$ which is $\eta_{k}^{\mathrm{CP}}= \pm i$ (see Ref. [65]). From Eq.(2.51) we have

$$
U_{\mathrm{CP}} \nu_{k L}(x) U_{\mathrm{CP}}^{-1}=\eta_{k}^{\mathrm{CP}} \gamma_{0} \nu_{k R}\left(x_{\mathrm{P}}\right) .
$$

This relation and Eqs.(2.35), (2.40) and (2.50) imply that the CP parity of the Majorana field $\nu_{k}$ is 95 97]

$$
\eta_{k}^{\mathrm{CP}}=i \rho_{k}
$$

Indeed, we have

$$
U_{\mathrm{CP}} N_{L}(x) U_{\mathrm{CP}}^{-1}=U^{\dagger} i \gamma_{0} \mathcal{C}{\overline{n_{L}}}^{T}\left(x_{\mathrm{P}}\right)=U^{\dagger} U^{*} i \gamma_{0} \mathcal{C}{\overline{N_{L}}}^{T}\left(x_{\mathrm{P}}\right)=i \rho \gamma_{0} N_{R}\left(x_{\mathrm{P}}\right)
$$

\subsection{Majorana mass term}

If only left-handed neutrino flavour fields $\nu_{\ell L}(\ell=e, \mu, \tau)$ enter into the Lagrangian we can write down the mass term [23, 98]

$$
\mathcal{L}^{\mathrm{M}}=-\frac{1}{2} \sum_{\ell, \ell^{\prime}} \overline{\left(\nu_{\ell L}\right)^{c}} M_{\ell \ell^{\prime}}^{L} \nu_{\ell^{\prime} L}
$$

where $M^{L}$ is a symmetric complex matrix (see previous section). Then we have for the mixing

$$
\nu_{\ell L}=\sum_{k=1}^{3} U_{\ell k} \nu_{k L},
$$

where $\nu_{k}$ is the field of Majorana neutrinos with mass $m_{k}$. In this case the number of massive Majorana neutrinos is equal to the number of lepton flavours (three). Note that the generation of the Majorana mass term (2.55) requires an enlargement of the scalar sector of the Minimal Standard Model [99]: with a Higgs triplet like in the Majoron models Ref. 91 93] Majorana masses are obtained at the tree level, whereas radiative generation is possible at the 1-loop level with a singly charged Higgs singlet (plus an additional Higgs doublet) [31, 100 104]) or at the 2-loop level with a doubly charged Higgs singlet (plus an additional singly charged scalar) 105. 
Since the Majorana condition (2.36) does not allow the rephasing of the neutrino fields, only three of the six phases in the $3 \times 3$ mixing matrix $U$ can be absorbed into the charged lepton fields in the charged current (2.19). Therefore, in the Majorana case the mixing matrix contains three CP-violating phases [28, 29, 106 in contrast to the single CP-violating phase of the Dirac case discussed in Section 2.1. However, the

additional CP-violating phases in the Majorana case have no effect on neutrino oscillations in vacuum [28, 29, 106] (see Section 3) as well as in matter [107].

\subsection{The one-generation case}

Let us consider the Dirac-Majorana mass term in the simplest case of one generation. We have

$$
\begin{aligned}
\mathcal{L}^{\mathrm{D}+\mathrm{M}} & =-\frac{1}{2} m_{L} \overline{\left(\nu_{L}\right)^{c}} \nu_{L}-m_{\mathrm{D}} \overline{\nu_{R}} \nu_{L}-\frac{1}{2} m_{R} \overline{\nu_{R}}\left(\nu_{R}\right)^{c}+\text { h.c. } \\
& =-\frac{1}{2} m_{R} \overline{\left(n_{L}\right)^{c}} M n_{L}+\text { h.c. }
\end{aligned}
$$

with

$$
n_{L} \equiv\left(\begin{array}{c}
\nu_{L} \\
\left(\nu_{R}\right)^{c}
\end{array}\right), \quad M \equiv\left(\begin{array}{cc}
m_{L} & m_{\mathrm{D}} \\
m_{\mathrm{D}} & m_{R}
\end{array}\right) .
$$

For simplicity we assume CP invariance in the lepton sector (see Subsection 2.2). In this case $m_{L}, m_{\mathrm{D}}$ and $m_{R}$ are real parameters. In order to diagonalize the matrix $M$, let us write it in the form

$$
M=\frac{1}{2} \operatorname{Tr} M+M^{0}=\frac{1}{2}\left(m_{L}+m_{R}\right)+M^{0},
$$

with

$$
M^{0}=\left(\begin{array}{cc}
-\frac{1}{2}\left(m_{R}-m_{L}\right) & m_{\mathrm{D}} \\
m_{\mathrm{D}} & \frac{1}{2}\left(m_{R}-m_{L}\right)
\end{array}\right) .
$$

The eigenvalues of the matrix $M^{0}$ are

$$
m_{1,2}^{0}=\mp \frac{1}{2} \sqrt{\left(m_{R}-m_{L}\right)^{2}+4 m_{\mathrm{D}}^{2}} .
$$

Furthermore, we have

$$
M^{0}=\mathcal{O} m^{0} \mathcal{O}^{T},
$$

where $m^{0}=\operatorname{diag}\left(m_{1}^{0}, m_{2}^{0}\right)$ and $\mathcal{O}$ is the orthogonal matrix

$$
\mathcal{O}=\left(\begin{array}{rr}
\cos \vartheta & \sin \vartheta \\
-\sin \vartheta & \cos \vartheta
\end{array}\right)
$$

with the mixing angle $\vartheta$ given by

$$
\cos 2 \vartheta=\frac{m_{R}-m_{L}}{\sqrt{\left(m_{R}-m_{L}\right)^{2}+4 m_{\mathrm{D}}^{2}}}, \quad \sin 2 \vartheta=\frac{2 m_{D}}{\sqrt{\left(m_{R}-m_{L}\right)^{2}+4 m_{\mathrm{D}}^{2}}} .
$$


Hence, the matrix $M$ can be written as

$$
M=\mathcal{O} m^{\prime} \mathcal{O}^{T},
$$

where $m^{\prime}=\operatorname{diag}\left(m_{1}^{\prime}, m_{2}^{\prime}\right)$ and

$$
m_{1,2}^{\prime}=\frac{1}{2}\left[\left(m_{R}+m_{L}\right) \mp \sqrt{\left(m_{R}-m_{L}\right)^{2}+4 m_{\mathrm{D}}^{2}}\right] .
$$

The eigenvalues of the matrix $M$ are real but can have positive or negative sign. Let us write them as

$$
m_{k}^{\prime}=m_{k} \rho_{k},
$$

where $m_{k}=\left|m_{k}^{\prime}\right|$ and $\rho_{k}$ is the sign of the $k^{\text {th }}$ eigenvalue of the matrix $M$. As shown in Eq.(2.53), the CP parity of the Majorana field $\nu_{k}$ with definite mass $m_{k}$ is $\eta_{k}^{\mathrm{CP}}=i \rho_{k}$. The relation (2.65) can be written in the form

$$
M=\left(U^{\dagger}\right)^{T} \hat{m} U^{\dagger},
$$

with $\hat{m}=\operatorname{diag}\left(m_{1}, m_{2}\right)$ and

$$
U^{\dagger} \equiv \sqrt{\rho} \mathcal{O}^{T},
$$

with $\rho=\operatorname{diag}\left(\rho_{1}, \rho_{2}\right)$. Using now the general formulas obtained in Section 2.2, we have the mixing relation

$$
\left(\begin{array}{c}
\nu_{L} \\
\left(\nu_{R}\right)^{c}
\end{array}\right)=U\left(\begin{array}{c}
\nu_{1 L} \\
\nu_{2 L}
\end{array}\right)
$$

with

$$
U=\left(\begin{array}{rr}
\left(\sqrt{\rho_{1}}\right)^{*} \cos \vartheta & \left(\sqrt{\rho_{2}}\right)^{*} \sin \vartheta \\
-\left(\sqrt{\rho_{1}}\right)^{*} \sin \vartheta & \left(\sqrt{\rho_{2}}\right)^{*} \cos \vartheta
\end{array}\right) .
$$

Therefore, the three parameters $m_{L}, m_{\mathrm{D}}, m_{R}$ are related with the mixing angle $\vartheta$ and the neutrino masses $m_{k}$ by the relations (2.64) and (2.66), (2.67). The signs of the eigenvalues of $M$ determine the CP parities of the massive Majorana fields $\nu_{k}$.

In the framework of $\mathrm{CP}$ conservation, the relations obtained so far are general. In the following part of this section we consider some particular cases with special physical significance.

\subsection{The see-saw mechanism}

Let us consider the Dirac-Majorana mass term (2.57) and assume [32 34 that $m_{L}=0$, $m_{\mathrm{D}} \simeq m^{f}$, where $m^{f}$ is the mass of a quark or a charged lepton of the same generation, and $m_{R} \simeq \mathcal{M} \gg m^{f}$. In this case, from the relations (2.64), (2.66) and (2.67) we have

$$
\begin{aligned}
& \vartheta \simeq \frac{m^{f}}{\mathcal{M}} \ll 1, \\
& m_{1} \simeq \frac{\left(m^{f}\right)^{2}}{\mathcal{M}} \ll m^{f}, \quad \rho_{1}=-1, \\
& m_{2} \simeq \mathcal{M}, \quad \rho_{2}=1 .
\end{aligned}
$$


These relations imply that approximately

$$
\nu_{L}=-i \nu_{1 L}, \quad\left(\nu_{R}\right)^{c}=\nu_{2 L},
$$

and the Majorana fields $\nu_{1}, \nu_{2}$ are connected with the fields $\nu_{L}$ and $\nu_{R}$ by

$$
\nu_{1}=i\left[\nu_{L}-\left(\nu_{L}\right)^{c}\right], \quad \nu_{2}=\nu_{R}+\left(\nu_{R}\right)^{c} .
$$

The mechanism which we consider here is called see-saw mechanism 32 34]. It is based on the assumption that the conservation of the total lepton number $L$ is violated by the right-handed Majorana mass term at the scale $\mathcal{M}$ that is much larger than the scale of the electroweak symmetry breaking. Several models which implement the seesaw mechanism are possible (see, for example, [31, 66, 68] and references therein) and the scale $\mathcal{M}$ depends on the model. This scale could be as low as the $\mathrm{TeV}$ scale (for example, in left-right symmetric models [31,108, 109]) or an intermediate scale, or as high as the grand unification scale $\sim 10^{15} \mathrm{GeV}$ or even the Plank scale $\sim 10^{19} \mathrm{GeV}$. The great attractiveness of the see-saw model lies in the fact that, through the relation (2.73), it gives an explanation of the smallness of neutrino masses with respect to the masses of other fundamental fermions. f

In the case of three generations the see-saw mechanism leads to a spectrum of masses of Majorana particles with three light neutrino masses $m_{k}$ and three very heavy masses $M_{k}(k=1,2,3)$ of the order of the scale of violation of the lepton numbers. This is realized if the mass matrix (2.32) has $M^{L}=0$,

$$
M^{\mathrm{D}+\mathrm{M}} \equiv\left(\begin{array}{cc}
0 & \left(M^{\mathrm{D}}\right)^{T} \\
M^{\mathrm{D}} & M^{R}
\end{array}\right),
$$

and $M^{R}$ is such that all its eigenvalues are much bigger than the elements of $M^{\mathrm{D}}$. In this case the mass matrix is block-diagonalized (up to corrections of order $\left(M^{R}\right)^{-1} M^{\mathrm{D}}$ ) by the unitary transformation

$$
W^{T} M^{\mathrm{D}+\mathrm{M}} W \simeq\left(\begin{array}{cc}
M_{\text {light }} & 0 \\
0 & M_{\text {heavy }}
\end{array}\right),
$$

with

$$
W \simeq\left(\begin{array}{cc}
1-\frac{1}{2}\left(M^{\mathrm{D}}\right)^{\dagger}\left(M^{R}\left(M^{R}\right)^{\dagger}\right)^{-1} M^{\mathrm{D}} & \left(M^{\mathrm{D}}\right)^{\dagger}\left(M^{R}\right)^{\dagger^{-1}} \\
-\left(M^{R}\right)^{-1} M^{\mathrm{D}} & 1-\frac{1}{2}\left(M^{R}\right)^{-1} M^{\mathrm{D}}\left(M^{\mathrm{D}}\right)^{\dagger}\left(M^{R}\right)^{\dagger^{-1}}
\end{array}\right) .
$$

The matrices for the light and heavy masses are given by [112, [113]

$$
M_{\text {light }} \simeq-\left(M^{\mathrm{D}}\right)^{T}\left(M^{R}\right)^{-1} M^{\mathrm{D}}, \quad M_{\text {heavy }} \simeq M^{R} .
$$

The mass eigenvalues of the light neutrinos are determined by the specific form of $M^{\mathrm{D}}$ and $M^{R}$. Note that in left-right symmetric models and in $\mathrm{SO}(10)$ models the matrix $M^{L}$ in the big matrix $M^{\mathrm{D}+\mathrm{M}}(2.32$ ) can be important (see, e.g., Ref. [114]).

Two simple possibilities are discussed in the literature (see Refs. [115, 116]):

\footnotetext{
${ }^{6}$ Note that the see-saw mechanism can also be realized for Dirac neutrinos 110, 111 .
} 
1. If $M^{R}=\mathcal{M} I$, where $I$ is the identity matrix, one obtains the quadratic see-saw,

$$
M_{\text {light }} \simeq-\frac{\left(M^{\mathrm{D}}\right)^{T} M^{\mathrm{D}}}{\mathcal{M}},
$$

and the light neutrino masses are given by

$$
m_{k}=\frac{\left(m_{k}^{f}\right)^{2}}{\mathcal{M}} \quad(k=1,2,3),
$$

where $m_{k}^{f}$ is the mass of a quark or a charged lepton of the $k^{\text {th }}$ generation. In this case the neutrino masses $m_{k}$ scale as the squares of the masses $m_{k}^{f}$ :

$$
m_{1}: m_{2}: m_{3}=\left(m_{1}^{f}\right)^{2}:\left(m_{2}^{f}\right)^{2}:\left(m_{3}^{f}\right)^{2} .
$$

2. If $M^{R}=\frac{\mathcal{M}}{\mathcal{M}_{\mathrm{D}}} M_{\mathrm{D}}$, where $\mathcal{M}_{\mathrm{D}}$ characterizes the scale of $M_{\mathrm{D}}$, one obtains the linear see-saw (see, for example, Ref. [117]),

$$
M_{\text {light }} \simeq-\frac{\mathcal{M}_{\mathrm{D}}}{\mathcal{M}} M^{\mathrm{D}},
$$

and the light neutrino masses are given by

$$
m_{k}=\frac{\mathcal{M}_{\mathrm{D}}}{\mathcal{M}} m_{k}^{f} \quad(k=1,2,3) .
$$

In this case the neutrino masses $m_{k}$ scale as the masses $m_{k}^{f}$ :

$$
m_{1}: m_{2}: m_{3}=m_{1}^{f}: m_{2}^{f}: m_{3}^{f} .
$$

Let us stress that in any case the see-saw mechanism implies the hierarchical relation

$$
m_{1} \ll m_{2} \ll m_{3}
$$

for the three light Majorana neutrino masses.

\subsection{Effective Lagrangians}

In the Standard Model without right-handed singlet neutrino fields there are no renormalizable interactions that give masses to the neutrinos after the spontaneous breaking of the $\mathrm{SU}(2)_{L} \times \mathrm{U}(1)_{Y}$ symmetry with the Higgs doublet mechanism. However, there is a general belief that the Standard Model is the low-energy manifestation of a more complete theory [118, 119] (for reviews see Refs. [31, 120]). The effect of this new theory is to induce in the Lagrangian of the Standard Model non-renormalizable interactions which preserve the $\mathrm{SU}(2)_{L} \times \mathrm{U}(1)_{Y}$ symmetry above the electroweak symmetry breaking scale, but violate the conservation of lepton and baryon numbers (see Ref. [121] and references therein). These non-renormalizable interactions are operators of dimension $d>4$ and 
must be multiplied by coupling constants that have dimension $\mathcal{M}^{4-d}$, where $\mathcal{M}$ is a mass scale characteristic of the new theory. It is clear that the dominant effects at low energies are produced by the operators with lowest dimension.

In the Standard Model the lepton number non-conserving operator with minimum dimension that can generate a neutrino mass is] [122 127]

$$
\frac{1}{\mathcal{M}} \sum_{\ell, \ell^{\prime}} \frac{g_{\ell \ell^{\prime}}}{2}\left(L_{\ell}^{T} \mathcal{C}^{-1} \sigma_{2} \vec{\sigma} L_{\ell^{\prime}}\right)\left(\phi^{T} \sigma_{2} \vec{\sigma} \phi\right)+\text { h.c. }
$$

where $g$ is a $3 \times 3$ matrix of coupling constants, $\vec{\sigma}$ are the Pauli matrices, $L_{\ell}$ are the standard leptonic doublets and $\phi$ is the standard Higgs doublet:

$$
L_{\ell} \equiv\left(\begin{array}{c}
\nu_{\ell L} \\
\ell_{L}
\end{array}\right) \quad(\ell=e, \mu, \tau), \quad \phi \equiv\left(\begin{array}{c}
\varphi^{+} \\
\varphi^{0}
\end{array}\right)
$$

The effective operator in Eq.(2.88) has dimension five and the coupling constant is proportional to $\mathcal{M}^{-1}$.

When $\mathrm{SU}(2)_{L} \times \mathrm{U}(1)_{Y}$ is broken by the vacuum expectation value $v / \sqrt{2}$ of $\varphi^{0}$, the effective interaction (2.88) generates the Majorana mass term

$$
\mathcal{L}^{\mathrm{M}}=\frac{1}{2} \frac{v^{2}}{\mathcal{M}} \sum_{\ell, \ell^{\prime}} g_{\ell \ell^{\prime}} \nu_{\ell L}^{T} \mathcal{C}^{-1} \nu_{\ell^{\prime} L}+\text { h.c. }=-\frac{1}{2} \frac{v^{2}}{\mathcal{M}} \sum_{\ell, \ell^{\prime}} \overline{\left(\nu_{\ell L}\right)^{c}} g_{\ell \ell^{\prime}} \nu_{\ell^{\prime} L}+\text { h.c. }
$$

where $v \simeq 246 \mathrm{GeV}$. In this mass term there is a suppression factor $v / \mathcal{M}$ which is responsible for the smallness of the neutrino masses [122, 123.

\subsection{Maximal mixing}

The expression (2.64) for the mixing angle $\vartheta$ implies that the mixing is maximal, i.e., $\vartheta=\pi / 4$, if $m_{R}=m_{L}$. In this case, assuming that $m_{L} \geq\left|m_{\mathrm{D}}\right|$, the Majorana neutrino masses are given by

$$
m_{1,2}=m_{L} \mp m_{\mathrm{D}} \text {. }
$$

The fields $\nu_{L}$ and $\left(\nu_{R}\right)^{c}$ are connected with the fields $\nu_{1}$ and $\nu_{2}$ by the relations

$$
\nu_{L}=\frac{1}{\sqrt{2}}\left(\nu_{1 L}+\nu_{2 L}\right), \quad\left(\nu_{R}\right)^{c}=\frac{1}{\sqrt{2}}\left(-\nu_{1 L}+\nu_{2 L}\right)
$$

The massive Majorana fields are given by

$$
\nu_{1,2}=\frac{1}{\sqrt{2}}\left\{\left[\nu_{L}+\left(\nu_{L}\right)^{c}\right] \mp\left[\nu_{R}+\left(\nu_{R}\right)^{c}\right]\right\} .
$$

\footnotetext{
${ }^{7}$ This operator can be written also as

$$
\frac{1}{\mathcal{M}} \sum_{\ell, \ell^{\prime}} g_{\ell \ell^{\prime}}\left(L_{\ell}^{T} \sigma_{2} \phi\right) \mathcal{C}^{-1}\left(\phi^{T} \sigma_{2} L_{\ell^{\prime}}\right)+\text { h.c. }
$$
}


If $m_{R}=m_{L}=0$ and $m_{\mathrm{D}}>0$, the mass term (2.57) is simply a Dirac mass term. Applying Eqs.(2.64) and (2.66), we obtain

$$
\vartheta=\frac{\pi}{4}, \quad m_{1,2}^{\prime}=\mp m_{\mathrm{D}} .
$$

Using Eq.(2.71) for the mixing matrix $U$, one can see that the fields $\nu_{L}$ and $\left(\nu_{R}\right)^{c}$ are connected to the Majorana fields $\nu_{1}$ and $\nu_{2}$ by the relations

$$
\nu_{L}=\frac{1}{\sqrt{2}}\left(-i \nu_{1 L}+\nu_{2 L}\right), \quad\left(\nu_{R}\right)^{c}=\frac{1}{\sqrt{2}}\left(i \nu_{1 L}+\nu_{2 L}\right)
$$

where $\nu_{1,2}$ are Majorana fields with the same mass $m_{1}=m_{2}=m_{\mathrm{D}}$ and with CP parities $\eta_{1}^{\mathrm{CP}}=-i$ and $\eta_{2}^{\mathrm{CP}}=+i$ (see Eq.(2.53) $)$. Eq.(2.93) implies for a Dirac field

$$
\nu=\frac{1}{\sqrt{2}}\left(-i \nu_{1}+\nu_{2}\right)
$$

Thus we arrive at the well-known result that a Dirac field can always be represented as an equal mixture of two Majorana fields with the same mass and opposite CP parities.

One can see this result also directly:

$$
\nu=-\frac{i}{\sqrt{2}}\left(i \frac{\nu-\nu^{c}}{\sqrt{2}}\right)+\frac{1}{\sqrt{2}} \frac{\nu+\nu^{c}}{\sqrt{2}}=-\frac{i}{\sqrt{2}} \nu_{1}+\frac{1}{\sqrt{2}} \nu_{2},
$$

where

$$
\nu_{1}=i \frac{\nu-\nu^{c}}{\sqrt{2}}, \quad \nu_{2}=\frac{\nu+\nu^{c}}{\sqrt{2}}
$$

are Majorana fields.

Finally, there is the possibility that $\left|m_{L}\right|,\left|m_{R}\right| \ll m_{\mathrm{D}}$ but at least one of the parameters $m_{L, R}$ is non-zero. In this case Eqs.(2.95) and (2.96) are approximately valid and $\nu_{1,2}$ are two Majorana neutrinos with opposite CP parities and almost degenerate masses given by

$$
m_{1,2} \simeq m_{\mathrm{D}} \mp \frac{1}{2}\left(m_{L}+m_{R}\right) .
$$

The field $\nu$ is called in this case a pseudo-Dirac neutrino field [62, 65, 128 130].

\section{Neutrino oscillations in vacuum}

\subsection{The general formalism}

If there is neutrino mixing, the left-handed components of the neutrino fields $\nu_{\alpha L}(\alpha=$ $\left.e, \mu, \tau, s_{1}, s_{2}, \ldots\right)$ are unitary linear combinations of the left-handed components of the $n$ (Dirac or Majorana) neutrino fields $\nu_{k}(k=1, \ldots, n)$ with masses $m_{k}$ :

$$
\nu_{\alpha L}=\sum_{k=1}^{n} U_{\alpha k} \nu_{k L}
$$


The number $n$ of massive neutrinos is 3 for the Dirac mass term discussed in Section 2.1 and for the Majorana mass term discussed in Section 2.3, in which cases there are only the three active flavour neutrinos. The number $n$ of massive neutrinos is more than three in the case of a Dirac-Majorana mass term discussed in Section 2.2 with a mixing of both, active and sterile neutrinos. In general, the number of light massive neutrinos can be more than three. We enumerate the neutrino masses in such a way that

$$
m_{1} \leq m_{2} \leq m_{3} \leq \ldots \leq m_{n}
$$

In this section we will consider in detail the phenomenon of neutrino oscillations in vacuum which is implied by the mixing relation (3.1).

If all neutrino mass differences are small, a state of a flavour neutrino $\nu_{\alpha}$ produced in a weak process (as the $\pi^{+} \rightarrow \mu^{+} \nu_{\mu}$ decay, nuclear beta-decays, etc.) with momentum $p \gg m_{k}$ is described by the coherent superposition of mass eigenstates (for a discussion of the quantum mechanical problems of neutrino oscillations see Refs. [61, 62, 131 146])

$$
\left|\nu_{\alpha}\right\rangle=\sum_{k=1}^{n} U_{\alpha k}^{*}\left|\nu_{k}\right\rangle
$$

Here $\left|\nu_{k}\right\rangle$ is the state of a neutrino with negative helicity, mass $m_{k}$ and energy

$$
E_{k}=\sqrt{p^{2}+m_{k}^{2}} \simeq p+\frac{m_{k}^{2}}{2 p} .
$$

Let us assume that at the production point and at time $t=0$ the state of a neutrino is described by Eq.(3.3). According to the Schrödinger equation the mass eigenstates $\left|\nu_{k}\right\rangle$ evolve in time with the phase factors $\exp \left(-i E_{k} t\right)$ and at the time $t$ at the detection point we have

$$
\left|\nu_{\alpha}\right\rangle_{t}=\sum_{k=1}^{n} U_{\alpha k}^{*} e^{-i E_{k} t}\left|\nu_{k}\right\rangle
$$

Neutrinos are detected by observing weak interaction processes. Expanding the state (3.5) in the basis of flavour neutrino states $\left|\nu_{\beta}\right\rangle$, we obtain

$$
\left|\nu_{\alpha}\right\rangle_{t}=\sum_{\beta} \mathcal{A}_{\nu_{\alpha} \rightarrow \nu_{\beta}}(t)\left|\nu_{\beta}\right\rangle
$$

where

$$
\mathcal{A}_{\nu_{\alpha} \rightarrow \nu_{\beta}}(t)=\sum_{k=1}^{n} U_{\beta k} e^{-i E_{k} t} U_{\alpha k}^{*}
$$

is the amplitude of $\nu_{\alpha} \rightarrow \nu_{\beta}$ transitions at the time $t$ at a distance $L \simeq t$. Consequently, the probability of this transition is given by

$$
P_{\nu_{\alpha} \rightarrow \nu_{\beta}}=\left|\mathcal{A}_{\nu_{\alpha} \rightarrow \nu_{\beta}}(t)\right|^{2}=\left|\sum_{k=1}^{n} U_{\beta k} e^{-i E_{k} t} U_{\alpha k}^{*}\right|^{2}
$$


This formula has a very simple interpretation. $U_{\alpha k}^{*}$ is the amplitude to find the neutrino mass eigenstate $\left|\nu_{k}\right\rangle$ with energy $E_{k}$ in the state of the flavour neutrino $\left|\nu_{\alpha}\right\rangle$, the factor $\exp \left(-i E_{k} t\right)$ gives the time evolution of the mass eigenstate and, finally, the term $U_{\beta k}$ gives the amplitude to find the flavour neutrino state $\left|\nu_{\beta}\right\rangle$ in the mass eigenstate $\left|\nu_{k}\right\rangle$. We want to remark that weak interaction processes responsible for neutrino production and detection involve active neutrinos. Therefore, strictly speaking, in the derivation of the Eqs. 3.7) and (3.8) the indices $\alpha$ and $\beta$ take only active flavour indices. However, transitions into sterile neutrinos can be revealed through neutral current neutrino experiments (disappearance of active neutrinos). In this sense, the formulas (3.7) and (3.8) have a meaning also for transitions between active and sterile states.

Notice that in order to have a non-negligible active-sterile transition probability the sterile fields must have a mixing with the light neutrino mass eigenfields the number of which must be more than three. Such a possibility is phenomenologically given by the Dirac-Majorana mass term (2.21), but it is not realized in the simple see-saw scheme discussed in Section 2.5, where the scale of the right-handed Majorana mass term is large. However, the see-saw scenario can be modified by additional assumptions to include light sterile neutrinos ("singular see-saw" [147], "universal see-saw [148, 149]).

At this point a remark concerning the unitarity of the mixing matrix $U$ is at order. If some of the mass eigenstates are so heavy that they are not produced in the standard weak processes then these mass eigenstates will not occur in the flavour state $\left|\nu_{\alpha}\right\rangle(3.3)$. Let us assume that the first $n^{\prime}$ mass eigenstates are light $\left(n^{\prime}<n\right)$. Consequently, only that part of $U$ plays a role in neutrino oscillations where $k \leq n^{\prime}$. In the following we will always assume that in the situation described here we can confine ourselves to an $n^{\prime} \times n^{\prime}$ submatrix of $U$ which is unitary to a good approximation (see, e.g., Ref. [150]). This is realized in the see-saw mechanism with a sufficiently large right-handed scale. In the further discussion we will drop the distinction between $n$ and $n^{\prime}$.

From the relation (3.1) it follows that the state describing a flavour antineutrino $\bar{\nu}_{\alpha}$ is given by

$$
\left|\bar{\nu}_{\alpha}\right\rangle=\sum_{k=1}^{n} U_{\alpha k}\left|\bar{\nu}_{k}\right\rangle .
$$

Thus the amplitude of $\bar{\nu}_{\alpha} \rightarrow \bar{\nu}_{\beta}$ transitions is given by

$$
\mathcal{A}_{\bar{\nu}_{\alpha} \rightarrow \bar{\nu}_{\beta}}(t)=\sum_{k=1}^{n} U_{\beta k}^{*} e^{-i E_{k} t} U_{\alpha k} .
$$

Notice that the amplitude for antineutrino transitions differs from the corresponding amplitude (3.7) for neutrinos only by the exchange $U \rightarrow U^{*}$.

Using the unitarity relation

$$
\sum_{k=1}^{n} U_{\beta k} U_{\alpha k}^{*}=\delta_{\alpha \beta}
$$




\begin{tabular}{|cccc|}
\hline Experiment & $L(\mathrm{~m})$ & $E(\mathrm{MeV})$ & $\Delta m^{2}\left(\mathrm{eV}^{2}\right)$ \\
\hline Reactor SBL & $10^{2}$ & 1 & $10^{-2}$ \\
Reactor LBL & $10^{3}$ & 1 & $10^{-3}$ \\
Accelerator SBL & $10^{3}$ & $10^{3}$ & 1 \\
Accelerator LBL & $10^{6}$ & $10^{3}$ & $10^{-3}$ \\
Atmospheric & $10^{7}$ & $10^{3}$ & $10^{-4}$ \\
Solar & $10^{11}$ & 1 & $10^{-11}$ \\
\hline
\end{tabular}

Table 3.1. Order of magnitude estimates of the values of $\Delta m^{2}$ which can be probed in reactor shortbaseline (SBL) and long-baseline (LBL), accelerator SBL and LBL, atmospheric and solar neutrino oscillation experiments. Note, however, that the energies and distances of the various types of experiments can vary in a wide range and only some representative values are given in this table.

the probability (3.8) can be written as

$$
P_{\nu_{\alpha} \rightarrow \nu_{\beta}}=\left|\delta_{\alpha \beta}+\sum_{k=2}^{n} U_{\beta k} U_{\alpha k}^{*}\left[\exp \left(-i \frac{\Delta m_{k 1}^{2} L}{2 E}\right)-1\right]\right|^{2}
$$

where $\Delta m_{k j}^{2} \equiv m_{k}^{2}-m_{j}^{2}$ and the ultrarelativistic approximation (3.4) has been used. Thus the probability of $\nu_{\alpha} \rightarrow \nu_{\beta}$ transitions depends on the elements of the mixing matrix, on $n-1$ independent mass-squared differences and on the parameter $L / E$, whose range is determined by the experimental setup.

If there is no mixing $(U=I)$ or/and $\Delta m_{k 1}^{2} L / E \ll 1$ for all $k=2, \ldots, n$, there are no transitions $\left(P_{\nu_{\alpha} \rightarrow \nu_{\beta}}=\delta_{\alpha \beta}\right)$. Neutrino transitions can be observed only if neutrino mixing takes place and at least one $\Delta m^{2}$ satisfies the condition

$$
\Delta m^{2} \gtrsim \frac{E}{L}
$$

In this inequality, $\Delta m^{2}$ is the neutrino mass-squared in $\mathrm{eV}^{2}, L$ is the distance between neutrino source and detector in $\mathrm{m}(\mathrm{km})$ and $E$ is neutrino energy in $\mathrm{MeV}(\mathrm{GeV})$. Thus, the larger the value of the parameter $L / E$, the smaller are values of $\Delta m^{2}$ which can be probed in an experiment. The inequality (3.13) allows to estimate (for large mixing angles) the sensitivity to the parameter $\Delta m^{2}$ of different types of neutrino oscillation experiments. These estimates are presented in Table 3.1. Let us stress that this table gives only a very rough idea of the sensitivity to $\Delta m^{2}$. For example, in the LSND short-baseline (SBL) experiment antineutrino energies are between 20 and $60 \mathrm{MeV}$, the distance is approximately $30 \mathrm{~m}$ and the minimal value of $\Delta m^{2}$ probed in this experiment is $4 \times 10^{-2} \mathrm{eV}^{2}$.

\footnotetext{
${ }^{8}$ We use the symbol $\Delta m^{2}$ for a generic mass-squared difference. Hence $\Delta m^{2}$ can be any of the $\Delta m_{k j}^{2}$ 's.
} 
The probability (3.8) and the corresponding one for antineutrinos are invariant under the phase transformation

$$
U_{\alpha k} \rightarrow e^{-i \varphi_{\alpha}} U_{\alpha k} e^{i \psi_{k}}
$$

Therefore, it is clear that the probabilities of $\nu_{\alpha} \rightarrow \nu_{\beta}$ and $\bar{\nu}_{\alpha} \rightarrow \bar{\nu}_{\beta}$ transitions do not depend [28, 29, 106] on the Majorana CP-violating phases discussed at the end of Section 2.3 and it is not possible to distinguish the Dirac and Majorana cases by the observation of neutrino oscillations.

Comparing the expressions (3.7) and (3.10) for the transition amplitudes of neutrinos and antineutrinos we see that $\mathcal{A}_{\nu_{\alpha} \rightarrow \nu_{\beta}}(t)=\mathcal{A}_{\bar{\nu}_{\beta} \rightarrow \bar{\nu}_{\alpha}}(t)$. Therefore, for the transition probabilities we have

$$
P_{\nu_{\alpha} \rightarrow \nu_{\beta}}=P_{\bar{\nu}_{\beta} \rightarrow \bar{\nu}_{\alpha}} .
$$

This relation is a consequence of CPT invariance inherent in any local field theory [151]. From the equality (3.15) it follows that the neutrino and antineutrino survival probabilities are equal:

$$
P_{\nu_{\alpha} \rightarrow \nu_{\alpha}}=P_{\bar{\nu}_{\alpha} \rightarrow \bar{\nu}_{\alpha}} .
$$

On the other hand, the transition probabilities of neutrinos and antineutrinos in general are different for $\alpha \neq \beta$. They are equal only if there is CP invariance in the lepton sector. In fact, in the case of massive Dirac neutrinos the phases of the neutrino fields and of the charged lepton fields can be chosen in such a way that the mixing matrix $U$ is real. In the case of Majorana neutrinos, CP invariance implies that (see Eq.(2.50))

$$
U_{\alpha k}^{*}=U_{\alpha k} \rho_{k},
$$

with $\rho_{k}=-i \eta_{k}^{\mathrm{CP}}= \pm 1$, where $\eta_{k}^{\mathrm{CP}}$ is the CP parity of the Majorana neutrinos with mass $m_{k}$ (see Eq.(2.53)). Thus, in the Dirac as well as in the Majorana case, CP invariance implies that

$$
P_{\nu_{\alpha} \rightarrow \nu_{\beta}}=P_{\bar{\nu}_{\alpha} \rightarrow \bar{\nu}_{\beta}} .
$$

\subsection{Oscillations in the two-neutrino case}

The results of neutrino oscillation experiments are usually analysed under the simplest assumption of oscillations between two neutrino types. In this case, for the transition probability (3.12) we get

$$
P_{\nu_{\alpha} \rightarrow \nu_{\beta}}=\left|\delta_{\alpha \beta}+U_{\beta 2} U_{\alpha 2}^{*}\left[\exp \left(-i \frac{\Delta m^{2} L}{2 E}\right)-1\right]\right|^{2},
$$

where $\Delta m^{2}=m_{2}^{2}-m_{1}^{2}$ and $\alpha, \beta$ are $e, \mu$, or $\mu, \tau$, etc... Thus in the simplest case of transitions between two neutrino types the probability is determined only by the elements of $U$ which connect flavour neutrinos with $\nu_{2}$ (or $\nu_{1}$ ). It is obvious that phases drop out in the expression (3.19). This is an illustration of Eq.(3.14) and of the fact that no information about $\mathrm{CP}$ violation can be obtained in the case of transitions between only two neutrino types. If we put $U_{\alpha 2}=\sin \vartheta$ then we have $U_{\beta 2}=\cos \vartheta$ and the transition 
and survival probabilities are given by the following standard expressions valid for both, neutrinos and antineutrinos:

$$
\begin{aligned}
& P_{\substack{\nu_{\alpha} \rightarrow \nu_{\beta} \\
\nu^{-}}}=\frac{1}{2} \sin ^{2} 2 \vartheta\left(1-\cos \frac{\Delta m^{2} L}{2 E}\right) \quad(\alpha \neq \beta) \\
& P_{(-) \rightarrow(-)}=P_{\substack{(-))(-) \\
\nu_{\alpha} \rightarrow \nu_{\alpha}}}=1-P_{\substack{(-) \\
\nu_{\alpha} \rightarrow \nu_{\beta}}} .
\end{aligned}
$$

The transition probability (3.20) can be written in the form

$$
\underset{\substack{(-) \\ \nu_{\alpha} \rightarrow \nu_{\beta}}}{P_{-}}=\frac{1}{2} \sin ^{2} 2 \vartheta\left(1-\cos 2.53 \frac{\Delta m^{2} L}{E}\right) \quad(\alpha \neq \beta) .
$$

where $L$ is the source - detector distance expressed in $\mathrm{m}(\mathrm{km}), E$ is the neutrino energy in $\mathrm{MeV}(\mathrm{GeV})$ and $\Delta m^{2}$ is the neutrino mass-squared difference in $\mathrm{eV}^{2}$. Thus, the transition probability is a periodic function of $L / E$. This phenomenon is called neutrino oscillations. The amplitude of oscillations is $\sin ^{2} 2 \vartheta$ and the oscillation length is given by

$$
L^{\mathrm{osc}}=\frac{4 \pi E}{\Delta m^{2}} \simeq 2.48 \frac{E(\mathrm{MeV})}{\Delta m^{2}\left(\mathrm{eV}^{2}\right)} \mathrm{m} .
$$

The condition (3.13) can be rewritten in the form

$$
L^{\mathrm{osc}} \lesssim L
$$

Therefore, neutrino oscillations can be observed if the oscillation length is not much larger than the source - detector distance $L$.

The oscillatory behaviour of the transition probability (3.20) with $\sin ^{2} 2 \vartheta=1$ is shown in Fig. 3.1, where we have plotted it as a function of $\Delta m^{2} L / 4 \pi E=L / L^{\text {osc }}$. The grey line represents the transition probability $(3.20)$, whereas, in order to demonstrate the effect of energy averaging, the black line represents the transition probability (3.20) averaged over a Gaussian energy distribution with mean value $E$ and standard deviation $\sigma=E / 10$. The averaged probability is the measurable quantity in neutrino oscillation experiments. One can see that the averaging over the energy spectrum practically reduces the probability to the constant $1-\sin ^{2} 2 \vartheta / 2$ for $L \gg L^{\text {osc }}$.

The expressions (3.20) and (3.21) are usually employed in analyses of the data of neutrino oscillation experiments. In many SBL experiments with neutrinos from reactors and accelerators, no indication in favour of neutrino oscillations was found. The data of these experiments give an upper bound for the transition probability which implies an excluded region in the space of the parameters $\Delta m^{2}$ and $\sin ^{2} 2 \vartheta$. A typical exclusion plot is presented in Fig. 3.2 152]. This plot shows the exclusion curves in the $\nu_{\mu} \rightarrow \nu_{\tau}$ channel obtained in the CDHS [153], FNAL E531 [154, CHARM II [155], CCFR [156], CHORUS [157] and NOMAD [158] experiments. The excluded region lies on the right of the curves.

The two most stringent exclusion curves in Fig. 3.2 have been obtained in the CHORUS [157] and NOMAD [158] experiments, which are operating at CERN using the 


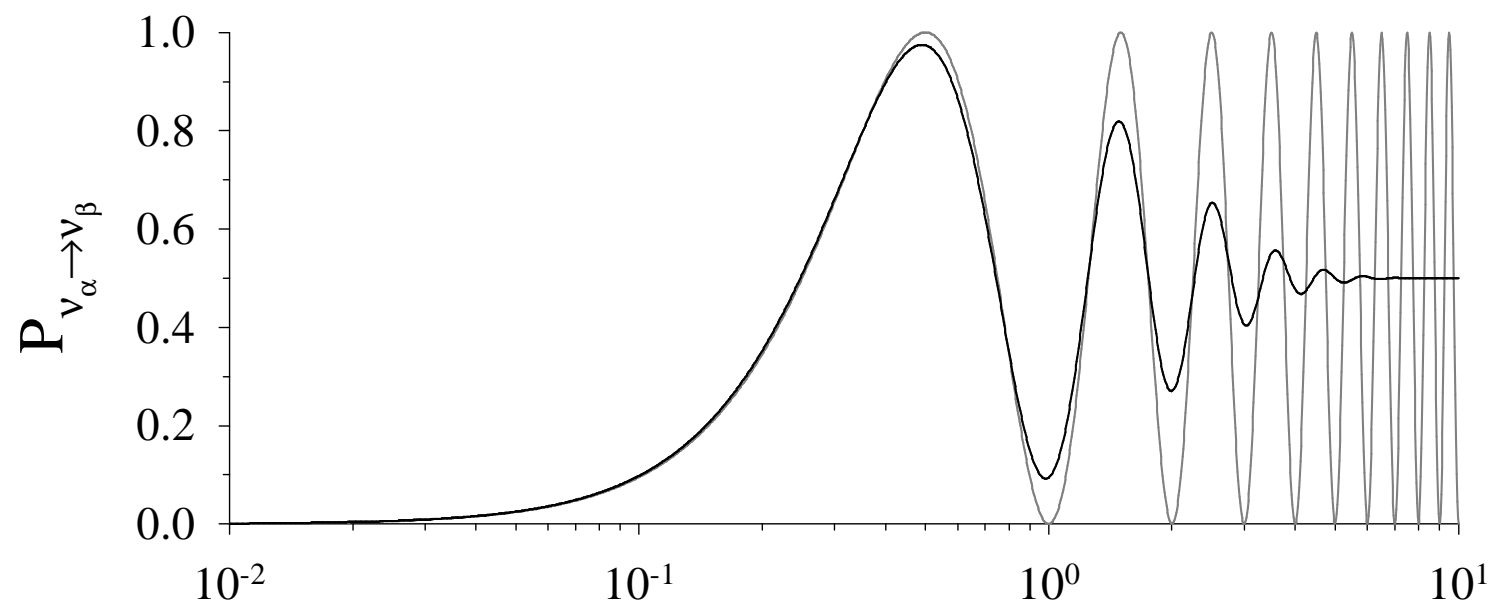

$\Delta m^{2} L / 4 \pi E$

Figure 3.1. Transition probability for $\sin ^{2} 2 \vartheta=1$ as a function of $\Delta m^{2} L / 4 \pi E=L / L^{\text {osc }}$, where $L^{\text {osc }}$ is the oscillation length. The grey line represents the transition probability (3.20) and the black line represents the same transition probability averaged over a Gaussian energy spectrum with mean value $E$ and standard deviation $\sigma=E / 10$.

neutrino beam from the SPS (with an average energy of about $30 \mathrm{GeV}$ ). $800 \mathrm{~kg}$ of emulsions are used in the CHORUS experiment as target. The production and decay of $\tau$ 's in the emulsion is searched for. In the NOMAD experiment a magnetic detector is used and the production of $\tau$ 's is identified with kinematical criteria.

Figure 3.3 shows the exclusion curves obtained in the CHOOZ [159], Gösgen [160, Krasnoyarsk [161] and Bugey [162] reactor $\bar{\nu}_{e} \rightarrow \bar{\nu}_{e}$ experiments. The region allowed by the results of the Kamiokande atmospheric neutrino experiment [49] (see Section 5.1) is also depicted in this figure. The Gösgen, Krasnoyarsk and Bugey experiments are SBL reactor experiments, whereas the recent $\mathrm{CHOOZ}$ experiment is the first LBL reactor experiment. In this experiment the detector ( 5 tons of liquid scintillator loaded with $\mathrm{Gd}$ ) is at the distance of about $1 \mathrm{~km}$ from the CHOOZ power station, which has two water reactors with a total thermal power of $8.5 \mathrm{GW}$. The antineutrinos are detected through the observation of the reaction

$$
\bar{\nu}_{e}+p \rightarrow e^{+}+n
$$

(the photons from annihilation of the positron and the delayed photons from the capture of neutron by $\mathrm{Gd}$ are detected). No indications in favour of neutrino oscillations were found in the CHOOZ experiment. The ratio $R$ of the numbers of measured antineutrino events and of expected antineutrino events in the case of absence of neutrino oscillations in the CHOOZ detector is 159

$$
R=0.98 \pm 0.04 \pm 0.04 .
$$

Since the average value of $L / E$ in the CHOOZ experiment is approximately $300(\langle E\rangle \simeq$ $3 \mathrm{MeV}, L \simeq 1000 \mathrm{~m}$ ), in this experiment it is possible to probe the value of the relevant neutrino mass-squared difference down to $10^{-3} \mathrm{eV}^{2}$. 


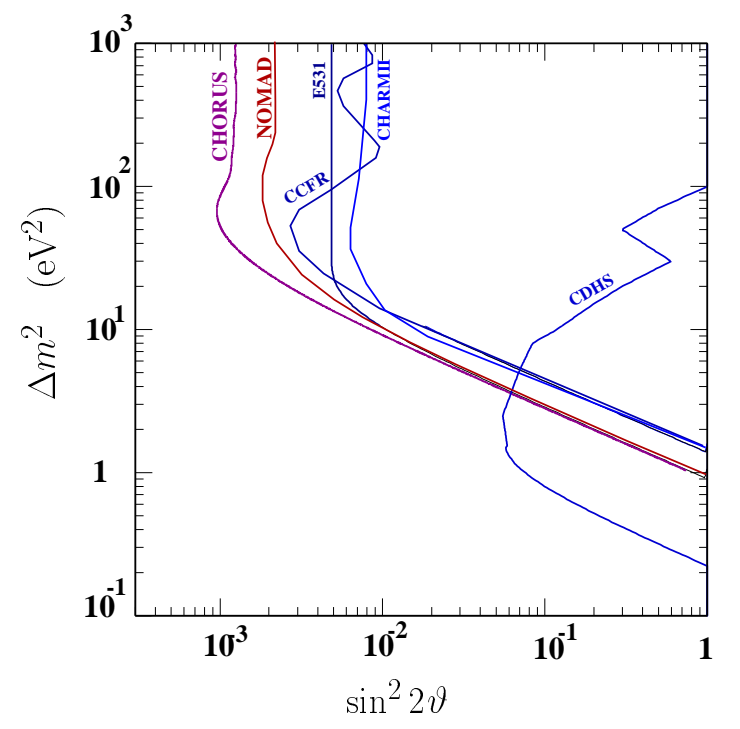

Figure 3.2. Exclusion curves (90\% CL) in the $\nu_{\mu} \rightarrow \nu_{\tau}$ channel obtained in the CDHS 153, FNAL E531 154], CHARM II 155], CCFR 156], CHORUS [157] and NOMAD [158] experiments.

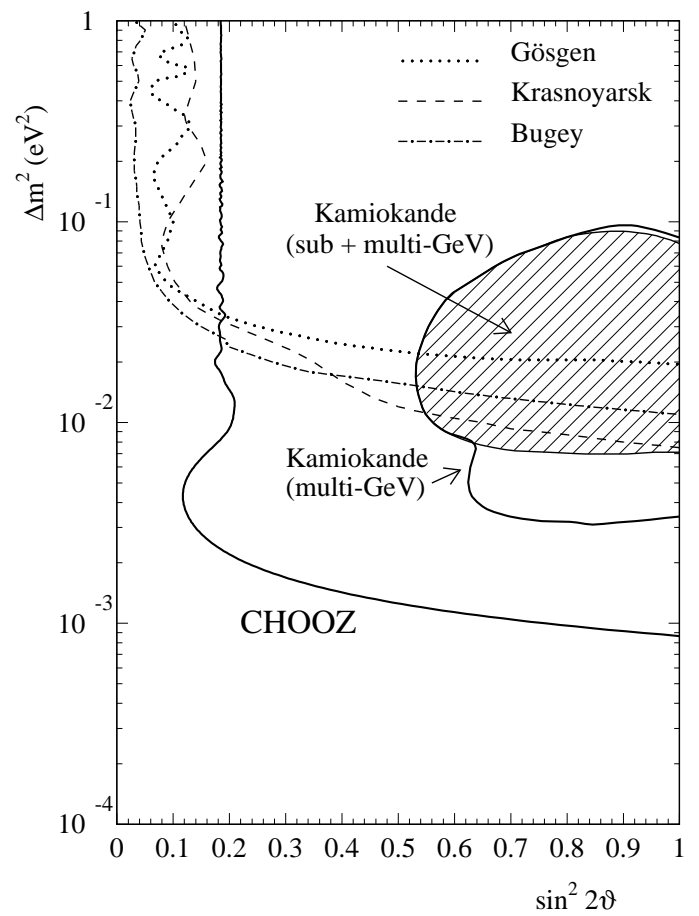

Figure 3.3. Exclusion curves (90\% CL) in the $\bar{\nu}_{e} \rightarrow \bar{\nu}_{e}$ channel obtained in the CHOOZ 159], Gösgen 160], Krasnoyarsk 161 and Bugey 1162 experiments. The shadowed region is allowed by the results of the Kamiokande atmospheric neutrino experiment 49].

With the help of the expression (3.22), it is possible to understand qualitatively the general features of exclusion curves. In the region of large $\Delta m^{2}$ such that the oscillation length is much smaller than the source - detector distance $L$, the cosine in the expression (3.22) oscillates very rapidly as a function of the neutrino energy $E$. Since in practice all neutrino beams have an energy spectrum and the neutrino sources and detectors are extended in space, only the average transition probability

$$
\left\langle\underset{\substack{(-))(-) \\ \nu_{\alpha} \rightarrow \nu_{\beta}}}{ }\right\rangle=\frac{1}{2} \sin ^{2} 2 \vartheta
$$

can be determined in the region of large $\Delta m^{2}$. The average probability is independent from $\Delta m^{2}$ and, therefore, from an experimental upper bound $\left.\underset{\substack{\nu_{\alpha} \rightarrow \nu_{\beta} \\ P_{(-)}}}{ }\right\rangle_{0}$ on $\left.\underset{\substack{(-))(-) \\ \nu_{\alpha} \rightarrow \nu_{\beta}}}{ }\right\rangle$ one obtains the vertical-line part of the exclusion curve.

At

$$
\Delta m^{2} \simeq \frac{\pi}{2} \frac{\langle E\rangle}{1.27\langle L\rangle},
$$

where $\langle E\rangle$ is the average energy and $\langle L\rangle$ is the average distance, the parameter $\sin ^{2} 2 \vartheta$ has the minimal value

$$
\left(\sin ^{2} 2 \vartheta\right)_{\min }=\left\langle\underset{\nu_{\alpha} \rightarrow \nu_{\beta}}{\left(P_{-)}\right)}\right\rangle_{0}
$$


on the boundary curve. Note that we are using here and in the rest of the section the same units as in Eq.(3.22).

Typically, the upper bound $\left\langle P_{(-))(-)}\right\rangle_{\nu_{\alpha} \rightarrow \nu_{\beta}}$ is much less than one. Then, in the region where $\sin ^{2} 2 \vartheta$ is large the expression (3.22) for the transition probability can be approximated by

$$
P_{\substack{(-) \\ \nu_{\alpha} \rightarrow \nu_{\beta}}} \simeq \sin ^{2} 2 \vartheta\left(1.27 \frac{\Delta m^{2} L}{E}\right)^{2}
$$

and in this region the boundary curve in the exclusion plot is given by

$$
\Delta m^{2} \simeq \frac{\sqrt{\left\langle P_{(-)(-)}\right\rangle_{0}}}{1.27 \sqrt{\sin ^{2} 2 \vartheta\left\langle L^{2}\right\rangle\left\langle E^{-2}\right\rangle}} .
$$

Therefore, this part of the exclusion curve is a straight line in the $\log \sin ^{2} 2 \vartheta-\log \Delta m^{2}$ plot as can be seen from Fig. 3.2. From Eq.(3.31) it follows that the minimal value of the parameter $\Delta m^{2}$ that can be probed by an experiment is

$$
\Delta m^{2} \simeq \frac{\sqrt{\left\langle P_{(-))(-)}\right\rangle_{0}}}{1.27 \sqrt{\left\langle L^{2}\right\rangle\left\langle E^{-2}\right\rangle}}
$$

It corresponds to $\sin ^{2} 2 \vartheta=1$.

\section{Neutrino oscillations and transitions in matter}

\subsection{The effective Hamiltonian for neutrinos in matter}

It has been pointed out by Wolfenstein 163 and by Mikheyev and Smirnov 164 that the neutrino oscillation pattern in vacuum can get significantly modified by the passage of neutrinos through matter because of the effect of coherent forward scattering. This effect can be described by an effective Hamiltonian. Starting with neutrino oscillations in vacuum, one can easily check that the transition probability (3.12) can be obtained by considering the evolution of the state vector $\psi$ of the neutrino types with the "Schrödinger equation"

$$
i \frac{\mathrm{d} \psi(x)}{\mathrm{d} x}=H_{\nu}^{\mathrm{vac}} \psi(x)=\frac{1}{2 E} U \hat{m}^{2} U^{\dagger} \psi(x),
$$

where $H_{\nu}^{\text {vac }}$ is the effective Hamiltonian for neutrino oscillations in vacuum with $U$ being the mixing matrix, $E$ the neutrino energy and $\hat{m}$ the diagonal neutrino mass matrix. The presence of matter will give a correction to Eq.(4.1). Note that the variable in Eq.(4.1) is not time but space and the components of $\psi(x)$ are given by the amplitudes denoted by $a_{\alpha}(x)$ for the neutrino types $\alpha=e, \mu, \tau, s$. The corresponding effective Hamiltonian for antineutrinos $H_{\bar{\nu}}^{\mathrm{vac}}$ is obtained from $H_{\nu}^{\mathrm{vac}}$ by the exchange $U \rightarrow U^{*}$. After the seminal work of Wolfenstein about neutrino oscillations in matter (see also Ref. [165]) and the discovery of the importance of $H_{\nu}^{\text {mat }}$, the analogue of $H_{\nu}^{\mathrm{vac}}$ in matter, for the solar neutrino 
problem [164] many rederivations of the effective matter Hamiltonian [166, 167] have been presented using a coupled system of Dirac equations [168, 169], field theory [170] or studying coherent forward scattering in more detail [171].

We want to sketch a derivation using the Dirac equation and following Ref. [169. The starting point in most derivations is the expectation value of the currents for isotropic non-relativistic matter given by [163, 164, 172]

$$
\left\langle\bar{f}_{L} \gamma_{\mu} f_{L}\right\rangle_{\mathrm{matter}}=\frac{1}{2} N_{f} \delta_{\mu 0},
$$

where $N_{f}$ is the number density of the particles represented by the field $f$. Note that the $\gamma_{5}$ term does not contribute for non-aligned spins [173]. Eq.(4.2) is a good approximation even for electrons in the core of the sun with a temperature $T \simeq 16 \times 10^{6} \mathrm{~K}$ [174, 175] and thus an average velocity of the electrons of around $10 \%$ of the velocity of light and corrections of order $k T / m_{e} \simeq 2.6 \times 10^{-3}$. Starting from the weak interaction Lagrangians (2.1) and (2.2) one gets for low-energy neutrino interactions of flavour $\ell$ with the background matter

$$
-\mathcal{L}_{\nu_{\ell}}^{\text {mat }}=\frac{G_{F}}{\sqrt{2}} \nu_{\ell}^{\dagger}\left(1-\gamma_{5}\right) \nu_{\ell} \sum_{f} N_{f}\left(\delta_{\ell f}+T_{3 f_{L}}-2 \sin ^{2} \theta_{W} Q_{f}\right),
$$

where $G_{F}$ is the Fermi coupling constant, $\theta_{W}$ the weak mixing angle, $T_{3 f_{L}}$ the eigenvalue of the field $f_{L}$ of the third component of the weak isospin $\left(T_{3 f_{R}}=0\right.$ in the Standard Model $)$ and $Q_{f}$ is the charge of $f$. In the matter Lagrangian (4.3) the charged current interaction is represented by the Kronecker symbol $\delta_{\ell f}$ saying that for neutrinos of flavour $\ell$ the charged current only contributes when background matter containing charged leptons of the same flavour is present. Concentrating now on realistic matter with electrons, protons and neutrons which is electrically neutral, i.e., $N_{e}=N_{p}$, and taking into account that we have $T_{3 e_{L}}=-T_{3 p_{L}}=T_{3 n_{L}}=-1 / 2$ and $Q_{e}=-Q_{p}=-1, Q_{n}=0$ for electrons, protons and neutrons, respectively,f] we get an effective Hamiltonian

$$
H_{\nu}^{D}=-i \vec{\alpha} \cdot \vec{\nabla}+\beta\left(M P_{L}+M^{\dagger} P_{R}\right)+\sqrt{2} G_{F} \operatorname{diag}\left(N_{e}-\frac{1}{2} N_{n},-\frac{1}{2} N_{n},-\frac{1}{2} N_{n}\right) P_{L}
$$

acting on the vector $\Psi(t, x)$ of the flavour neutrino wave functions, where we have defined $P_{L, R}=\left(\mathbf{1} \mp \gamma_{5}\right) / 2, \alpha_{j}=\gamma^{0} \gamma^{j}$ and $\beta=\gamma^{0} . M$ is the non-diagonal mass matrix. The last term in Eq.4.4) is called the matter potential term. Several remarks concerning the Hamiltonian (4.4), which is the point of departure for deriving the effective matter Hamiltonian in Refs. [168, 169], are at order:

1. $H_{\nu}^{D}$ has spinor and flavour indices, it leads thus to a system of Dirac equations coupled via the mass term.

2. The neutral current contributions of electrons and protons cancel for realistic matter because of opposite $T_{3 f_{L}}$ quantum numbers and electric charges (see discussion above before Eq.(4.4)).

\footnotetext{
${ }^{9}$ For protons and neutrons this is ensured by CVC (conserved vector current) for the $T_{3}$ part and conservation of the electromagnetic current.
} 
3. The Hamiltonian (4.4) is valid for Dirac neutrinos. For antineutrinos, $M$ is replaced by $M^{T}$ and the matter potential term of $H_{\bar{\nu}}^{D}$ has the opposite sign and the projector $P_{R}$ instead of $P_{L}$. This is a consequence of proceeding along the same lines as before but using the charge-conjugate fields to describe antineutrinos $\left(\bar{\nu}_{\ell} \gamma_{\rho} P_{L} \nu_{\ell}=-\overline{\nu_{\ell}^{c}} \gamma_{\rho} P_{R} \nu_{\ell}^{c}\right)$.

4. In the Majorana case, in order to obtain the Hamiltonian $H_{\nu}^{M}$ the antineutrino matter potential has to be added to $H_{\nu}^{D}$ and the neutrino field vector $\Psi$ is subject to the Majorana condition $\Psi=\mathcal{C} \gamma_{0}^{T} \Psi^{*}$. This condition is compatible with the time evolution governed by $H_{\nu}^{M}$ 169].

5. Sterile neutrinos can easily be incorporated into $H_{(-)}^{D}$ and $H_{\nu}^{M}$ by simply adding the entry 0 to the matter potential term. Note that the probability of active - active transitions does not depend on the neutron density $N_{n}$, which is, however, important for active - sterile transitions (see Table 1.1).

The essence of deriving an effective Hamiltonian for neutrino oscillations in matter is to get rid of the spinor indices in $H_{\nu}^{D}$ and to obtain an equation involving only the indices $\alpha$ for the different neutrino types as in the vacuum case (4.1). To this end, let us now assume that the neutrino propagation proceeds along the $x^{3} \equiv x$ axis, that the neutrino momentum $p>0$ corresponding to propagation in vacuum is much larger than the matter potentials and that the neutrinos are ultrarelativistic. Thus we consider a one-dimensional problem from now on. Defining a wave function $\phi$ via

$$
\Psi(t, x)=e^{i p x} \phi(x, t) \quad \text { with } \quad i \frac{\partial \phi}{\partial t}=\left(p \alpha_{3}+H_{\nu}^{D}\right) \phi,
$$

this wave function changes little over distances of the order of the de Broglie wave length of the neutrino. With respect to $\alpha_{3}$ we can decompose the Hamiltonian (4.4) into $H_{\nu}^{D}=$ $H_{\text {even }}+H_{\text {odd }}$ which are the parts commuting or anticommuting with $\alpha_{3} . H_{\text {odd }}$ consists solely of the mass term and $H_{\text {even }}$ contains the rest of $H_{\nu}^{D}$. With $p$ being a large parameter we can perform a Foldy - Wouthuysen transformation where we truncate the series at $1 / p$ leading to the Hamiltonian [169, 176]

$$
\begin{aligned}
H_{\mathrm{FW}, \nu}^{D} & =p \alpha_{3}+H_{\mathrm{even}}+\frac{1}{2 p} \alpha_{3} H_{\mathrm{odd}}^{2} \\
& =\alpha_{3}\left(p-i \frac{\partial}{\partial x}\right)+\sqrt{2} G_{F} \operatorname{diag}\left(N_{e}-\frac{1}{2} N_{n},-\frac{1}{2} N_{n},-\frac{1}{2} N_{n}\right) P_{L} \\
& +\frac{1}{2 p} \alpha_{3}\left(M^{\dagger} M P_{L}+M M^{\dagger} P_{R}\right),
\end{aligned}
$$

such that all terms in $H_{\mathrm{FW}, \nu}^{D}$ commute with $\alpha_{3}$ and, therefore, all matrices in $H_{\mathrm{FW}, \nu}^{D}$ with spinor indices can be diagonalized at the same time in order to separate positive and negative energy states. Denoting this unitary diagonalization matrix by $U_{0}$ we can achieve $U_{0} \alpha_{3} U_{0}^{\dagger}=\operatorname{diag}(1,1,-1,-1), U_{0} \frac{\mathbf{1}-\gamma_{5}}{2} U_{0}^{\dagger}=\operatorname{diag}(0,1,1,0)$ and $U_{0} \frac{\mathbf{1}+\gamma_{5}}{2} U_{0}^{\dagger}=\operatorname{diag}(1,0,0,1)$. Note that there are no transitions between left and right-handed states through $H_{\mathrm{FW}, \nu}^{D}$. 
For neutrinos with magnetic moments (electric dipole moments) the method described above would also yield the appropriate left-right transitions [169].

Going back to the Foldy - Wouthuysen transform of the wave function $\Psi$ instead of $\phi$ removes the $p$ from the Hamiltonian (4.6). The final step in deriving the effective matter Hamiltonian consists of considering stationary states and splitting off the plane wave part by

$$
\Psi_{\mathrm{FW}}(t, x)=e^{-i E(t-x)} \psi(x) .
$$

Then the wave function $\psi(x)$, taken for positive energies and left-handed neutrinos, fulfills the first order differential equation (4.1) with $H_{\nu}^{\mathrm{vac}}$ replaced by [163]

$$
H_{\nu}^{\mathrm{mat}}=\frac{1}{2 E}\left(M^{\dagger} M+2 \sqrt{2} E G_{F} \operatorname{diag}\left(N_{e}-\frac{1}{2} N_{n},-\frac{1}{2} N_{n},-\frac{1}{2} N_{n}\right)\right),
$$

correct up to order $1 / E$. This effective Hamiltonian is valid for left-handed Dirac neutrinos or Majorana neutrinos, whereas in the case of right-handed (Dirac) antineutrinos or right-handed Majorana neutrinos we have

$$
H_{\bar{\nu}}^{\text {mat }}=\frac{1}{2 E}\left(M^{T} M^{*}-2 \sqrt{2} E G_{F} \operatorname{diag}\left(N_{e}-\frac{1}{2} N_{n},-\frac{1}{2} N_{n},-\frac{1}{2} N_{n}\right)\right),
$$

because for (Dirac) antineutrinos one has to replace $M$ by $M^{T}$ in the Hamiltonian (4.6) and for Majorana neutrinos $M=M^{T}$ holds (see also points 3 and 1 after Eq.(4.4)). With

$$
M=U_{R} \hat{m} U_{L}^{\dagger} \quad \text { and } \quad U_{L} \equiv U
$$

only the left-handed mixing matrix $U$ enters and connection between Eqs.(4.8) and (4.1) is made. Thus in the final results (4.8) and (4.9) there is no distinction between the Dirac and Majorana case for ultrarelativistic neutrinos. This is an illustration of the fact that with neutrino oscillation experiments the Dirac or Majorana nature cannot be distinguished 98, 177.

The Hamiltonians (4.8) and (4.9) have been used to investigate neutrino oscillations in the sun, in the earth and in supernovae. In the following we will only be concerned with the first two subjects. Application limits of the neutrino evolution equations in matter have been discussed in Ref. 178. Elastic and inelastic neutrino scattering introduces quantum damping into the evolution equations which is proportional to the neutrino interaction rate [179]. In the sun and the earth this effect is negligible, in particular, for low energies, whereas in the early universe it is of crucial importance 179. Density fluctuations have been found to influence considerably neutrino propagation in the sun [180], however, more realistic considerations with helioseismic waves as density fluctuations show no observable effect on the solar neutrino problem discussed in terms of neutrino oscillations [181. Solar neutrinos are also influenced by their passage through the earth [182. 


\begin{tabular}{|c|cccc|}
\hline & $\nu_{e} \rightarrow \nu_{\mu, \tau}$ & $\nu_{e} \rightarrow \nu_{s}$ & $\nu_{\mu} \rightarrow \nu_{\tau}$ & $\nu_{\mu, \tau} \rightarrow \nu_{s}$ \\
\hline \hline$\frac{A}{2 \sqrt{2} E G_{F}}$ & $N_{e}$ & $N_{e}-\frac{1}{2} N_{n}$ & 0 & $-\frac{1}{2} N_{n}$ \\
\hline
\end{tabular}

Table 4.1. The list of matter densities relevant for two-neutrino oscillations (for the definition of $A$ see Eq.(4.12).

\subsection{The two-neutrino case and adiabatic transitions}

Let us now concentrate on left-handed neutrinos and specify the effective Hamiltonian (4.8) to two neutrino types. Thus for two-neutrino oscillations in matter with the definitions

$$
N\left(\nu_{\alpha}\right) \equiv \delta_{\alpha e} N_{e}-\frac{1}{2} N_{n} \quad(\alpha=e, \mu, \tau), \quad N\left(\nu_{s}\right) \equiv 0
$$

and

$$
A \equiv 2 \sqrt{2} G_{F} E\left(N\left(\nu_{\alpha}\right)-N\left(\nu_{\beta}\right)\right)
$$

the differential equation

$$
\begin{aligned}
i \frac{\mathrm{d}}{\mathrm{d} x}\left(\begin{array}{c}
a_{\alpha} \\
a_{\beta}
\end{array}\right)= & H_{\nu}^{\mathrm{mat}}\left(\begin{array}{c}
a_{\alpha} \\
a_{\beta}
\end{array}\right) \\
= & \frac{1}{4 E}\left\{\left[m_{1}^{2}+m_{2}^{2}+2 \sqrt{2} G_{F}\left(N\left(\nu_{\alpha}\right)+N\left(\nu_{\beta}\right)\right)\right]\left(\begin{array}{ll}
1 & 0 \\
0 & 1
\end{array}\right)\right. \\
& \left.+\left(\begin{array}{cc}
A-\Delta m^{2} \cos 2 \vartheta & \Delta m^{2} \sin 2 \vartheta \\
\Delta m^{2} \sin 2 \vartheta & -A+\Delta m^{2} \cos 2 \vartheta
\end{array}\right)\right\}\left(\begin{array}{l}
a_{\alpha} \\
a_{\beta}
\end{array}\right)
\end{aligned}
$$

has to be studied [163, 164, 172, 178, 183, 184, where $a_{\alpha}, a_{\beta}$ are the amplitudes for the neutrino types $\alpha, \beta(\alpha, \beta=e, \mu, \tau, s)$, respectively. The evolution equation for antineutrinos is obtained from Eq.(4.13) by $A \rightarrow-A$. In the two-neutrino case there is one $\Delta m^{2}=m_{2}^{2}-m_{1}^{2}$ and the mixing matrix

$$
U=\left(\begin{array}{rr}
\cos \vartheta & \sin \vartheta \\
-\sin \vartheta & \cos \vartheta
\end{array}\right)
$$

is real without loss of generality. The list of all possible matter densities which determine $A$ and occur in the different oscillation channels is given in Table 4.1. Evidently, $\nu_{\mu} \leftrightarrow \nu_{\tau}$ oscillations proceed as in vacuum because $A=0$ and the term proportional to the unit matrix in Eq.(4.13) has no effect on transitions. As mentioned before, in the rest of this section we will have in mind that Eq.(4.13) is applied to neutrino propagation in the sun and the earth.

Let us first define the eigenfunctions of the effective Hamiltonian as

$$
H_{\nu}^{\text {mat }} \psi_{m j}=E_{j} \psi_{m j} \quad \text { with } \quad \psi_{m 1}=\left(\begin{array}{r}
\cos \vartheta_{m} \\
-\sin \vartheta_{m}
\end{array}\right), \psi_{m 2}=\left(\begin{array}{c}
\sin \vartheta_{m} \\
\cos \vartheta_{m}
\end{array}\right)
$$


where the matter angle $\vartheta_{m}$ and the energy eigenvalues $E_{j}$ are functions of $x$. In the limit of vanishing matter density $\vartheta_{m}$ is identical with the vacuum angle $\vartheta$ (4.14). The eigenvalues $E_{j}$ and the matter angle are given by

$$
\begin{aligned}
E_{1,2}=\frac{1}{4 E}\left\{m_{1}^{2}+\right. & m_{2}^{2}+2 \sqrt{2} G_{F}\left(N\left(\nu_{\alpha}\right)+N\left(\nu_{\beta}\right)\right) \\
& \left.\mp \sqrt{\left(A-\Delta m^{2} \cos 2 \vartheta\right)^{2}+\left(\Delta m^{2} \sin 2 \vartheta\right)^{2}}\right\}
\end{aligned}
$$

and

$$
\tan 2 \vartheta_{m}=\frac{\tan 2 \vartheta}{1-\frac{A}{\Delta m^{2} \cos 2 \vartheta}}
$$

respectively. The general solution of the differential equation (4.13) can be represented as

$$
\psi(x)=\sum_{j=1}^{2} a_{j}(x) \psi_{m j}(x) \exp \left(-i \int_{x_{0}}^{x} \mathrm{~d} x^{\prime} E_{j}\left(x^{\prime}\right)\right)
$$

with the coefficients $a_{1,2}$ fulfilling

$$
\frac{\mathrm{d}}{\mathrm{d} x}\left(\begin{array}{c}
a_{1} \\
a_{2}
\end{array}\right)=\left(\begin{array}{cc}
0 & -\frac{\mathrm{d} \vartheta_{m}}{\mathrm{~d} x} \exp \left(i \int_{x_{0}}^{x} \mathrm{~d} x^{\prime} \Delta E\left(x^{\prime}\right)\right) \\
\frac{\mathrm{d} \vartheta_{m}}{\mathrm{~d} x} \exp \left(-i \int_{x_{0}}^{x} \mathrm{~d} x^{\prime} \Delta E\left(x^{\prime}\right)\right) & 0
\end{array}\right)\left(\begin{array}{l}
a_{1} \\
a_{2}
\end{array}\right)
$$

and $\Delta E \equiv E_{2}-E_{1}$.

The adiabatic solution is defined as an approximate solution where $\dot{a}_{j} \simeq 0(j=1,2) . \mathrm{T}$ Postponing the discussion of the question under which condition adiabaticity is fulfilled, we consider temporarily the case of an arbitrary number $n$ of neutrino flavours or types and define the mixing matrix in matter $U_{m}(x)$ via

$$
U_{m}(x)^{\dagger} H_{\nu}^{\mathrm{mat}} U_{m}(x)=\operatorname{diag}\left(E_{1}(x), \ldots, E_{n}(x)\right) \quad \text { with } \quad U_{m}(x)=\left(\psi_{m 1}, \ldots, \psi_{m n}\right),
$$

where the eigenvectors $\psi_{m j}$ generalize Eq.(4.15). One readily obtains the generalization of the vacuum oscillation amplitude Eq.(3.12) for neutrino oscillations in matter if the evolution of the neutrino wave function is adiabatic:

$$
\mathcal{A}_{\nu_{\alpha} \rightarrow \nu_{\beta}}^{\text {adiab }}=\sum_{j} U_{m}\left(x_{1}\right)_{\beta j} \exp \left(-i\left\{\delta_{j}+\int_{x_{0}}^{x_{1}} \mathrm{~d} x^{\prime} E_{j}\left(x^{\prime}\right)\right\}\right) U_{m}^{*}\left(x_{0}\right)_{\alpha j}
$$

In this formula neutrino production and detection happen at $x_{0}$ and $x_{1}$, respectively. The adiabatic phases $\delta_{j}$, which are defined by

$$
\delta_{j} \equiv-i \int_{x_{0}}^{x_{1}} \mathrm{~d} x^{\prime} \psi_{m j}\left(x^{\prime}\right)^{\dagger} \dot{\psi}_{m j}\left(x^{\prime}\right)
$$

\footnotetext{
${ }^{10}$ We use the dot above a symbol as abbreviation for $\frac{\mathrm{d}}{\mathrm{d} x}$.
} 
are necessary for the correct evolution of $\psi(x)$ in the adiabatic limit. Usually, they can be absorbed into the eigenvectors $\psi_{m j}$ [185], except for special matter densities, where these phases acquire a topological meaning, together with the presence of CP violation [186]. Note that for real vectors $\psi_{m j}$ one has $\psi_{m j}^{\dagger} \dot{\psi}_{m j}=0$ and therefore $\delta_{j}=0$. This is so in the two-dimensional case Eq.(4.15).

Evaluating Eq.4.21) for two neutrino types and assuming that an averaging over neutrino energies takes place such that

$$
\left\langle\exp \left(-i \int_{x_{0}}^{x_{1}} \mathrm{~d} x^{\prime} \Delta E\left(x^{\prime}\right)\right)\right\rangle_{\mathrm{av}}=0
$$

it is easy to show that the averaged two-neutrino survival probability can be written in the adiabatic case as

$$
\bar{P}_{\nu_{\alpha} \rightarrow \nu_{\alpha}}=\frac{1}{2}\left(1+\cos 2 \vartheta_{m}\left(x_{0}\right) \cos 2 \vartheta_{m}\left(x_{1}\right)\right) .
$$

Let us now derive a condition for adiabaticity in the two-neutrino case. The evolution of the neutrino state in matter is adiabatic if the right-hand side of Eq.(4.19) can be neglected. A formal solution of Eq.(4.19) is given by

$$
\left(\begin{array}{l}
a_{1}(x) \\
a_{2}(x)
\end{array}\right)=\sum_{k=0}^{\infty} \int_{x_{0}}^{x} \mathrm{~d} x^{\prime} J\left(x^{\prime}\right) \int_{x_{0}}^{x^{\prime}} \mathrm{d} x^{\prime \prime} J\left(x^{\prime \prime}\right) \cdots \int_{x_{0}}^{x^{(k-1)}} \mathrm{d} x^{(k)} J\left(x^{(k)}\right)\left(\begin{array}{l}
a_{1}\left(x_{0}\right) \\
a_{2}\left(x_{0}\right)
\end{array}\right),
$$

where $J$ denotes the matrix on the right-hand side of Eq.4.19). Defining the variable

$$
\alpha \equiv \frac{1}{2} \int_{x_{0}}^{x} \mathrm{~d} x^{\prime} \Delta E\left(x^{\prime}\right)
$$

and the adiabaticity parameter (as a function of $x$ ) [184, 187 189]

$$
\gamma(x) \equiv \frac{\Delta E}{2\left|\dot{\vartheta}_{m}\right|}
$$

one can determine an upper bound to the typical integral which occurs in all the terms of the sum (4.25) on the right end. In an interval $\left[y_{0}, y_{1}\right]$ where $\gamma$ is monotonous there is a value $y_{c}$ such that [190]

$$
\pm \int_{y_{0}}^{y_{1}} d y \dot{\vartheta}_{m} \cos 2 \alpha=\int_{\alpha\left(y_{0}\right)}^{\alpha\left(y_{1}\right)} d \alpha \frac{1}{\gamma} \cos 2 \alpha=\frac{1}{\gamma\left(y_{0}\right)} \int_{y_{0}}^{y_{c}} d \alpha \cos 2 \alpha+\frac{1}{\gamma\left(y_{1}\right)} \int_{y_{c}}^{y_{1}} d \alpha \cos 2 \alpha
$$

according to the second mean value theorem of integral calculus $\left(y_{0}<y_{c}<y_{1}\right)$. This consideration allows to put the bound

$$
\left|\int_{y_{0}}^{y_{1}} d y \dot{\vartheta}_{m} e^{2 i \alpha}\right| \leq \frac{2 \sqrt{2}}{\gamma_{\min }} \quad \text { with } \quad \gamma_{\min } \equiv \min _{y \in\left[y_{0}, y_{1}\right]} \gamma(y) .
$$

\footnotetext{
${ }^{11}$ The scalar product in Eq.(4.22) is imaginary for arbitrary vectors $\psi_{m j}(x)$ normalized to one $\forall x$, therefore, for real vectors it must be zero.
} 
Having found this bound, in the rest of the integrals in the terms in Eq.(4.25) one can simply take the absolute values of the integrands and one is left with integrations of the type $\mathrm{d} x\left|\dot{\vartheta}_{m}\right|=\left|\mathrm{d} \vartheta_{m}\right|$. With the refinement that the interval $\left[x_{0}, x\right]$ possibly has to be divided into several parts labelled by $a$ such that in each part interval $\gamma(y)$ is monotonous we can define

$$
\bar{\gamma}^{-1} \equiv \sum_{a} \gamma_{\min , a}^{-1}
$$

and obtain the exact bound

$$
\left|a_{j}(x)-a_{j}\left(x_{0}\right)\right| \leq\left.\frac{2 \sqrt{2}}{\bar{\gamma}}\left(\begin{array}{cc}
\cosh \Delta \Theta & \sinh \Delta \Theta \\
\sinh \Delta \Theta & \cosh \Delta \Theta
\end{array}\right)\left(\begin{array}{l}
\left|a_{1}\left(x_{0}\right)\right| \\
\left|a_{2}\left(x_{0}\right)\right|
\end{array}\right)\right|_{j},
$$

where the symbol $\left.\right|_{j}$ denotes the $j$-th element of the vector on the right-hand side of this inequality and $\Delta \Theta$ has a contribution $\left|\Delta \vartheta_{m}\right|$ for every interval where $\dot{\vartheta}_{m}$ has a definite sign. $\left(\Delta \vartheta_{m}\right.$ is the difference between the angle $\vartheta_{m}$ in the initial and the final point of such an interval.) Thus with the exact bound (4.31) we have found the following sufficient condition:

$$
\bar{\gamma} \gg 1 \Rightarrow \text { adiabatic evolution . }
$$

It is fulfilled if on the scale of the oscillation length in matter given by $1 / \Delta E$ the matter angle $\vartheta_{m}$ changes very little, i.e., $\left|\dot{\vartheta}_{m}\right| \ll \Delta E$. Eq. (4.31) allows to get an upper bound on the crossing or jumping probability from $\psi_{m 1}$ to $\psi_{m 2}$. Assuming the initial conditions $a_{1}\left(x_{0}\right)=1$ and $a_{2}\left(x_{0}\right)=0$ we get

$$
\left|a_{2}(x)\right| \leq \frac{2 \sqrt{2}}{\bar{\gamma}} \sinh \Delta \Theta
$$

which illustrates once more the importance of $\bar{\gamma}$ for adiabaticity.

\subsection{The resonance}

In the context of the solar neutrino problem the discovery that a resonance in the passage of $\nu_{e}$ through the sun is possible [164] gave a major boost to the investigation of the propagation of neutrinos in matter. The possibility of a resonance is most easily understood in the adiabatic approximation by looking at Eqs.4.17) and (4.24). For different neutrino masses we can always label them in such a way that $\Delta m^{2}>0$. If on the way from the creation point $x_{0}$ in matter of $\nu_{e}$ to a point $x_{1}$ in vacuum the neutrino passes through a point $x_{\text {res }}$ where the resonance condition

$$
A\left(x_{\mathrm{res}}\right)=\Delta m^{2} \cos 2 \vartheta
$$

is fulfilled then $\vartheta_{m}\left(x_{1}\right) \equiv \vartheta$ has to be between $0^{\circ}$ and $45^{\circ}$ (for $\nu_{e}$ the quantity $A$ is positive!) whereas according to Eq. (4.17) the matter angle $\vartheta_{m}\left(x_{0}\right)$ is found between $45^{\circ}$ and $90^{\circ} .12$ Consequently, the product of cosines in the $\nu_{e}$ survival probability (4.24) is

\footnotetext{
${ }^{12}$ Without loss of generality we consider $0^{\circ}<\vartheta<90^{\circ}$.
} 
negative and the probability to find a $\nu_{e}$ after the passage through the sun is less than $1 / 2$. The interesting phenomenon is that this can happen even for relatively small mixing angles, provided the matter potential at the production point is large enough such that there is a point $x_{\text {res }}$ where the condition (4.34) is fulfilled. Indeed, for $\vartheta$ close to $0^{\circ}$, one can even have $\vartheta_{m}\left(x_{1}\right)$ close to $90^{\circ}$ and thus $\bar{P}_{\nu_{e} \rightarrow \nu_{e}} \simeq 0$ (4.24). It has been shown that the resonance is also effective in a certain area in the $\Delta m^{2}-\sin ^{2} 2 \vartheta$ plane where the evolution of the neutrino state is non-adiabatic. For antineutrinos a resonance is possible if $45^{\circ}<\vartheta<90^{\circ}$.

If there is a resonance, then for reasonable matter densities the adiabaticity parameter (4.27) is smallest at the resonance and thus adiabaticity is most likely violated there. Therefore, considering $\gamma$ at the resonance, from Eq. (4.17) one easily computes $\left.\dot{\vartheta}_{m}\right|_{\text {res }}=$ $\dot{A}_{\text {res }} /\left(2 \sin 2 \vartheta \Delta m^{2}\right)$. A suitable measure of adiabaticity in the case of a resonance is thus given by 187,188

$$
\left.\gamma_{\mathrm{res}} \equiv \frac{\Delta E}{2\left|\dot{\vartheta}_{m}\right|}\right|_{\mathrm{res}}=\frac{\Delta m^{2} \sin ^{2} 2 \vartheta}{2 E \cos 2 \vartheta(|\dot{A}| / A)_{\mathrm{res}}} .
$$

In the solar interior the electron density is maximal in the center with $\left.N_{e}\right|_{\max } \simeq$ $100 \times N_{\text {avo }}$ where $N_{\text {avo }}=6.022 \times 10^{23} \mathrm{~cm}^{-3}$ and $\left.\left.N_{n}\right|_{\max } \simeq \frac{1}{2} N_{e}\right|_{\max }$ [174]. This leads to

$$
\left.2 \sqrt{2} G_{F} E N_{e}\right|_{\max } \simeq 1.5 \times 10^{-5}\left(\frac{E}{1 \mathrm{MeV}}\right) \mathrm{eV}^{2} .
$$

Considering the resonance condition (4.34) one can read off from this equation for which $\Delta m^{2}$ a resonance is possible. Whereas the density variation of $N_{e}$ and $N_{n}$ in the sun is smooth, in the earth due to its layered structure (lithosphere - mantle - core) the densities can be approximated as step functions [191, 192]. Furthermore, approximately $N_{n} \simeq N_{e}$ is valid in all layers. In the lithosphere, which is relevant for LBL neutrino oscillation experiments, the average mass density $\rho$ is around $3 \mathrm{~g} / \mathrm{cm}^{3}$. It increases to around $13 \mathrm{~g} / \mathrm{cm}^{3}$ in the core. Thus we can conveniently write

$$
2 \sqrt{2} G_{F} E N_{e} \simeq 2.3 \times 10^{-4} \mathrm{eV}^{2}\left(\frac{\rho}{3 \mathrm{~g} \mathrm{~cm}^{-3}}\right)\left(\frac{E}{1 \mathrm{GeV}}\right) .
$$

\subsection{Non-adiabatic neutrino oscillations in matter and crossing probabilities}

The analogue of the adiabatic formula (4.21) in terms of probabilities is given by

$$
P_{\nu_{\alpha} \rightarrow \nu_{\beta}}=\left|\sum_{j, k} U_{m}\left(x_{1}\right)_{\beta j} B\left(x_{1}, x_{0}\right)_{j k} U_{m}^{*}\left(x_{0}\right)_{\alpha k}\right|^{2}
$$

with

$$
\begin{aligned}
B\left(x_{1}, x_{0}\right) & =U_{m}^{\dagger}\left(x_{1}\right) P \exp \left\{-i \int_{x_{0}}^{x_{1}} \mathrm{~d} x H_{\nu}^{\mathrm{mat}}(x)\right\} U_{m}\left(x_{0}\right) \\
& =P \exp \left\{-i \int_{x_{0}}^{x_{1}} \mathrm{~d} x\left(\hat{E}-i U_{m}^{\dagger}(x) \dot{U}_{m}(x)\right)\right\}
\end{aligned}
$$


where $\hat{E}$ is the diagonal matrix of energy eigenvalues present in Eq. (4.20) (see Ref. [183]). $B\left(x_{1}, x_{0}\right)$ is a unitary $n \times n$ matrix which is diagonal in the adiabatic limit corresponding to $-i U_{m}^{\dagger}(x) \dot{U}_{m}(x) \simeq \operatorname{diag}\left(\delta_{1}, \ldots, \delta_{n}\right)$ (see Eq.(4.22) $)$.

Confining ourselves now to two neutrino types and having in mind neutrino production in matter and detection in vacuum we use the notation $\vartheta_{m}\left(x_{0}\right) \equiv \vartheta_{m}^{0}$ and $\vartheta_{m}\left(x_{1}\right)=$ $\vartheta$. With the crucial assumption that averaging over neutrino energies and the neutrino production region all terms other than probabilities can be dropped we get

$$
\left\langle B_{j k} B_{j^{\prime} k^{\prime}}^{*}\right\rangle_{\mathrm{av}}=\delta_{j j^{\prime}} \delta_{k k^{\prime}}\left|B_{j k}\right|^{2} \text { and }\left|B_{12}\right|^{2}=\left|B_{21}\right|^{2}=P_{c},\left|B_{11}\right|^{2}=\left|B_{22}\right|^{2}=1-P_{c},
$$

where $P_{c}$ is the crossing probability from $\psi_{m 1}$ to $\psi_{m 2}$ and in the second part of Eq. (4.40) unitarity has been used. Such averaging procedures have been shown to be effective in the context of solar neutrinos, e.g., in Refs. [193, 194]. Inserting the relations (4.40) into Eq.(4.38) specialized to the survival probability of $\nu_{e}$ one obtains after some algebra with trigonometric functions the Parke formula

$$
\bar{P}_{\nu_{e} \rightarrow \nu_{e}}=\frac{1}{2}+\left(\frac{1}{2}-P_{c}\right) \cos 2 \vartheta \cos 2 \vartheta_{m}^{0},
$$

which is the generalization of Eq.(4.24) [183, 189. This formula was first derived in the context of an exact solution of Eq.(4.13) for matter densities linear in $x$ [188, 195] (see also Refs. 187, 196]).

The probability $P_{c}$ can be estimated with the Landau - Zener method [196, 197. Following the derivation of Landau, the idea is to make an analytic continuation of the matter densities and therefore of the effective matter Hamiltonian (4.8) into the complex plane by $x \rightarrow z$ where $z$ is a complex variable. Then also the neutrino state $\psi(4.18)$ is analytic. Considering the energy eigenvalues $E_{1,2}(x)$ (4.16) as functions of $z$ we find two branching points $z_{0}$ and $z_{0}^{*}\left(\operatorname{Im} z_{0}>0\right)$ defined by the equation

$$
E_{1}\left(z_{0}\right)=E_{2}\left(z_{0}\right) \quad \Leftrightarrow A\left(z_{0}\right)=\Delta m^{2} e^{ \pm 2 i \vartheta}
$$

We consider $E_{1}(z)$ and $\psi_{m 1}(z)$ for definiteness and follow its evolution along a curve $C(z)$ which starts at a value $z$ in the vicinity of the real axis and goes along a negatively oriented loop around $z_{0}$ such that we end at the same point $z$ but now located on the second sheet.13 Along this path $E_{1}(z)$ changes into $E_{2}(z)$ 197 and $\psi_{m 1}(z)$ into $\psi_{m 2}(z)$. [4 The crucial observation is that, though $E_{j}(z)$ and $\psi_{m j}(z)(j=1,2)$ are two-valued analytic objects, the analytic continuation $\psi(z)$ of the solution (4.18) of the differential equation (4.13) is single-valued [198]. From this fact it can easily be shown with Eq.(4.18) that [198]

$$
\tilde{a}_{1}(z)=\exp \left(i \int_{C(z)} E_{1}(z) d z\right) a_{2}(z),
$$

\footnotetext{
${ }^{13}$ It is convenient to imagine cuts from $z_{0}$ to $z_{0}+i \infty$ and $z_{0}^{*}$ to $z_{0}^{*}-i \infty$ in the complex plane. Crossing these cuts, one moves from one sheet to the other.

${ }^{14}$ In general this change is accompanied by a factor of geometrical origin. It has no effect in our case for the jumping probability 198.
} 
where $\tilde{a}_{1}(z)$ is the analytic continuation of $a_{1}(x)$ in the vicinity of the real axis and then along $C(z)$. The Landau - Zener crossing probability is derived from the following consideration. Starting on the real axis at $x_{-} \ll x_{\text {res }}$, where the evolution of $\psi$ is adiabatic and where the boundary conditions $a_{1}\left(x_{-}\right)=1, a_{2}\left(x_{-}\right)=0$ hold, and going along a path in the complex plane which passes above $z_{0}$ and returning to the real axis at a point $x_{+} \gg x_{\text {res }}$ on the second sheet this path can be chosen such that the evolution in the complex variable $z$ is adiabatic because we have passed the resonance in safe distance and we have thus $\left|\tilde{a}_{1}\left(x_{+}\right)\right| \simeq 1$. On the other hand, we can go from $x_{+}$on the first sheet to the point $x_{+}$on the second sheet via $C\left(x_{+}\right)$and with Eq.4.43) we thus obtain

$$
P_{c}=\left|a_{2}\left(x_{+}\right)\right|^{2}=\left|\exp \left(-i \int_{C\left(x_{+}\right)} E_{1}(z) d z\right)\right|^{2},
$$

neglecting deviations of $\left|\tilde{a}_{1}\left(x_{+}\right)\right|$from 1 . From this formula we can read off that integrations along the real axis do not contribute to $P_{c}$. Because of analyticity, the path $C\left(x_{+}\right)$ can be deformed such that it goes along the real axis from $x_{+}$to $x_{\text {res }}$ from where it leads to $z_{0}$, circles around the branching point with infinitely small radius and then goes back to $x_{\text {res }}$ and $x_{+}$on the second sheet. In this way, we obtain from Eq.(4.44) the final result for the Landau - Zener crossing probability

$$
\ln P_{c}=-\frac{1}{E} \operatorname{Im} \int_{x_{\mathrm{res}}}^{z_{0}} d z\left[\left(A-\Delta m^{2} \cos 2 \vartheta\right)^{2}+\left(\Delta m^{2} \sin 2 \vartheta\right)^{2}\right]^{1 / 2} .
$$

To get this formula we have used the explicit form of the energies of the adiabatic states (4.16).

With the variable transformation $A=A(z)$ and $d z=d A / \frac{d A}{d z}$ it is easy to evaluate Eq.(4.45) for a linear density [172, 187 189] and is also possible for an exponential density [199. These calculations can be summarized by [183, 189]

$$
P_{c}=\exp \left(-\frac{\pi}{2} \gamma_{\mathrm{res}} F\right)
$$

with $F=1$ for the linear and $F=1-\tan ^{2} \vartheta$ for the exponential case. For an exponentially varying matter density the dependence of $P_{c}$ on $\vartheta$ is particularly simple because $\gamma_{\text {res }} F=$ $4 \delta \sin ^{2} \vartheta$ with $\delta \equiv \gamma_{\text {res }} \cos 2 \vartheta / \sin ^{2} 2 \vartheta$ which is independent of the vacuum mixing angle. For a more general discussion of the crossing probability (4.45) see Ref. [200].

Exact solutions of the differential equation (4.13) for neutrino oscillations in matter exist not only for the linear case [187, 188, 195, 196] in terms of Weber functions but also for the exponentially varying matter density in terms of Whittaker functions [193, 201]. This case is of particular importance for solar neutrinos because it approximates the real density variation in the sun. Further exact solutions are known for $A$ varying with $\tanh x$ [202] and $1 / x$ 189]. In Refs. [183, 189] a list of the factors $F$ for all these cases is given, calculated with the Landau - Zener formula (4.45).

The survival probability (4.41) with $P_{c}$ in the Landau - Zener approximation does not reproduce well the exact survival probability in the extremely non-adiabatic region 193, 195] (see also Refs. [172, 183, 189]). In these references the example of an extremely 
dense medium $\left(A \rightarrow \infty\right.$ and thus $\left.\vartheta_{m}^{0}=\pi / 2\right)$ with a sharp boundary to the vacuum is given. In this case the neutrino does not oscillate in the medium and therefore $\bar{P}_{\nu_{e} \rightarrow \nu_{e}}=$ $1-\frac{1}{2} \sin ^{2} 2 \vartheta$ stems purely from vacuum oscillations. This has to be compared with $P_{c}=1$, because of the jump in density one gets $\gamma_{\text {res }}=0$, inserted into Eq.4.41). Obviously, the resulting expression $\bar{P}_{\nu_{e} \rightarrow \nu_{e}}=\cos ^{2} \vartheta$ does not agree with the previous one.

A remedy of this deficiency was found in the framework of the exact solution for an exponentially varying matter density leading to the following modification [193 of the Landau - Zener crossing probability $P_{c}$ (4.45):

$$
P_{c}=\frac{\exp \left(-\frac{\pi}{2} \gamma_{\mathrm{res}} F\right)-\exp \left(-\frac{\pi}{2} \gamma_{\mathrm{res}} \frac{F}{\sin ^{2} \vartheta}\right)}{1-\exp \left(-\frac{\pi}{2} \gamma_{\mathrm{res}} \frac{F}{\sin ^{2} \vartheta}\right)}
$$

Numerical calculations for solar neutrinos with an exponential electron density using this formula agree very well with numerical integrations of the differential equation (4.13), typically the agreement is in the percent range for relevant mixing parameters 203]. It has been conjectured in Ref. 189 that the form (4.47) of $P_{c}$ holds for a wide class of matter density profiles. In any case, in the limit $\gamma_{\text {res }} \rightarrow 0$ one gets $P_{c} \rightarrow \cos ^{2} \vartheta$ which, when inserted into formula (4.41), correctly describes the survival probability in the above example of an extremely non-adiabatic evolution.

Concluding this section, we consider again an arbitrary number of neutrino flavours or types and envisage the interesting case where one of the eigenfunctions $(4.20)$ of $H_{\nu}^{\text {mat }}$ labelled by the index $\ell_{0}$ has an adiabatic evolution whereas the other part of $\psi$ has an arbitrary evolution. We assume exact adiabaticity for $\psi_{m \ell_{0}}$ which amounts to $B_{j \ell_{0}}=$ $B_{\ell_{0} k}=0$ for $j, k \neq \ell_{0}$. This allows to write

$$
P_{\nu_{\alpha} \rightarrow \nu_{\beta}}=\left(1-\left|U_{m}\left(x_{1}\right)_{\beta \ell_{0}}\right|^{2}\right)\left(1-\left|U_{m}\left(x_{0}\right)_{\alpha \ell_{0}}\right|^{2}\right) P_{\nu_{\alpha} \rightarrow \nu_{\beta}}^{\left(\ell_{0}\right)}+\left|U_{m}\left(x_{1}\right)_{\beta \ell_{0}}\right|^{2}\left|U_{m}\left(x_{0}\right)_{\alpha \ell_{0}}\right|^{2},
$$

where we have defined

$$
\begin{aligned}
P_{\nu_{\alpha} \rightarrow \nu_{\beta}}^{\left(\ell_{0}\right)} & =\left[\left(1-\left|U_{m}\left(x_{1}\right)_{\beta \ell_{0}}\right|^{2}\right)\left(1-\left|U_{m}\left(x_{0}\right)_{\alpha \ell_{0}}\right|^{2}\right)\right]^{-1}\left|\sum_{j, k \neq \ell_{0}} U_{m}\left(x_{1}\right)_{\beta j} B\left(x_{1}, x_{0}\right)_{j k} U_{m}^{*}\left(x_{0}\right)_{\alpha k}\right|^{2} \\
& \leq 1 .
\end{aligned}
$$

That $P_{\nu_{\alpha} \rightarrow \nu_{\beta}}^{\left(\ell_{0}\right)}$ is smaller than 1 follows from the Cauchy - Schwarz inequality and from the above assumption for $B$ because after dropping the row and the column labelled by $\ell_{0}$ in $B$ the remaining matrix is again unitary. Eq.4.49) and similar cases are useful if neutrino masses differing by orders of magnitude occur.

\section{Indications of neutrino oscillations}

\subsection{Atmospheric neutrino experiments}




\subsubsection{The atmospheric neutrino flux}

In 1912 it was discovered by V.F. Hess [204] in a manned balloon flight that the intensity of the ionizing radiation in the atmosphere as a function of the altitude did not conform with the idea that this ionization was caused by radioactive elements in the surface of the earth but rather pointed to an extraterrestrial origin. In the following decades, before the advent of accelerator physics, this radiation, which was called first "ultraradiation" and later baptized "cosmic rays" by R.A. Millikan, proved to be one of the most fruitful means for doing particle physics experiments. At the end of the first half of the 20th century such experiments had lead to the discovery of the positron, the pion and the muon and also the first particles with strangeness were found with cosmic rays [205]. Eventually, in the beginning of the fifties, proton beams from accelerators replaced the cosmic proton flux as an experimental tool. However, after many years where accelerator physics was dominating in particle physics, at the end of the 20th century cosmic rays play again a major role through atmospheric neutrinos which allow to use the whole globe as a neutrino physics laboratory and to probe neutrino mass-squared differences down to a few $10^{-4} \mathrm{eV}^{2}$. In this way convincing evidence for the existence of neutrino oscillations and thus for non-zero neutrino masses has been obtained [2].

In a simplified picture, the production of atmospheric neutrinos [206 218] proceeds in three steps [219]. In the first step the primary cosmic rays [219, 220] hit the nuclei in the atmosphere, thereby producing charged pions and kaons, either directly or via intermediate particles. In the second step, the decay of these particles gives rise to part of the atmospheric $\nu_{\mu}$ and $\bar{\nu}_{\mu}$ neutrino fluxes:

$$
\pi^{+} \rightarrow \mu^{+}+\nu_{\mu}, \pi^{-} \rightarrow \mu^{-}+\bar{\nu}_{\mu} \quad \text { and } \quad K^{+} \rightarrow \mu^{+}+\nu_{\mu}, K^{-} \rightarrow \mu^{-}+\bar{\nu}_{\mu} .
$$

In the third step, the $\nu_{e}$ and $\bar{\nu}_{e}$ fluxes and further $\nu_{\mu}$ and $\bar{\nu}_{\mu}$ fluxes are produced by

$$
\mu^{+} \rightarrow e^{+}+\nu_{e}+\bar{\nu}_{\mu} \quad \text { and } \quad \mu^{-} \rightarrow e^{-}+\bar{\nu}_{e}+\nu_{\mu}
$$

There is also a contribution to the neutrino fluxes from the decays

$$
K_{L} \rightarrow \pi^{+}+\ell^{-}+\bar{\nu}_{\ell} \text { and } K^{+} \rightarrow \pi^{0}+\ell^{+}+\nu_{\ell}
$$

with $\ell=e, \mu$ and the charge conjugate processes 215, 219,221] which does not conform with the simple 3-step picture. At low energies the most important process in Eq.(5.1) is the pion decay. The contribution of $K_{\ell 3}$ decays (5.3) to the neutrino fluxes is small [222, 223]. Let us now describe some useful details of the production of the atmospheric neutrino flux and its properties.

Cosmic rays: Galactic cosmic rays enter the solar system as an isotropic flux of particles. There is convincing evidence that the bulk of the radiation with energies less than $10^{6} \mathrm{GeV}$ comes from our galaxy. Though there is no conclusive proof of the origin of cosmic rays yet, plausible mechanisms range from material ejected by supernovae to interstellar medium accelerated in supernova shock waves 224]. Solar cosmic rays are 
emitted irregularly by major solar flares on the sun. Apart from electrons, primary cosmic rays consist of protons and bare nuclei (mostly He). Roughly speaking, the chemical composition of the galactic cosmic ray particles is given approximately by $90 \% \mathrm{H}, 9 \% \mathrm{He}$ and less than $1 \%$ heavier nuclei. However, the chemical composition varies with energy. At energies of around $100 \mathrm{MeV}$ per nucleon the particle number ratio $\mathrm{H} / \mathrm{He}$ is less than 5, it increases to 10 at $1 \mathrm{GeV}$ and is around 30 at $100 \mathrm{GeV}$ 225, 226]. The absolute flux of cosmic protons is not very large: it is of the order of $1000 / \mathrm{m}^{2} \times \sec \times$ sr for energies of a few $\mathrm{GeV}$ and above the atmosphere. As a function of the energy the proton flux above a few $\mathrm{GeV}$ is well described by the power law $E^{-2.7}$ until $10^{6} \mathrm{GeV}$ [225. Above an energy of $100 \mathrm{GeV}$ per nucleon the cosmic ray fluxes are less precisely known [225, 226].

Solar modulation: The solar cosmic rays (solar wind) are important in so far as they weaken and modulate the flux of galactic cosmic ray particles with the solar activity. The stronger the solar wind the more difficult it is for the low energy galactic cosmic rays to enter the solar sphere of influence. This modulation is noticeable for kinetic energies of around $10 \mathrm{GeV}$ per nucleon or less [215,224]. This variation of the primary cosmic ray flux with the approximate 11-year cycle of solar activity can be parameterized as a function of the neutron monitor at Mt. Washington [215]. It induces a corresponding modulation of the low energy atmospheric neutrino flux but its influence is small. The effect of the solar wind on cosmic ray particles depends on the rigidity of the nuclei which is defined by momentum/charge. It is strongest for cosmic ray particles with small rigidity. For neutrino energies $E_{\nu} \sim 2 \mathrm{GeV}$ it is practically negligible, for energies around $1 \mathrm{GeV}$ only appreciable at high geomagnetic latitudes because the geomagnetic cut-off admits cosmic ray particles with smaller rigidity there.

Geomagnetic cut-off: The geomagnetic field prevents primary cosmic particles with low rigidity from entering the atmosphere [215,218,227,228]. In the atmospheric neutrino flux calculations it is assumed that the momenta of the neutrinos have the same direction as the primary cosmic ray particles responsible for their production 215, 217]. This is a good approximation for $E_{\nu}>200 \mathrm{MeV}$ as shown in Ref. [229]. In a first approximation, for a given direction, there is a cut-off in rigidity for the primary cosmic ray particles below which such a particle cannot reach the top of the atmosphere in a vertical altitude of around $20 \mathrm{~km}$. The value of the geomagnetic cut-off can be obtained by a computer simulation with the help of the "backtracking technique". In this technique, to establish if a particle with a given charge and momentum coming from a certain direction can reach a point on top of the atmosphere, one integrates the equations of motion for a particle with opposite charge and reflected momentum starting from its final position. If the backtracked particle reaches infinity it is assumed that the trajectory is allowed. As infinity one can take a distance of around 10 times the radius of the earth where the geomagnetic field has decreased to the level of the interstellar magnetic field with a strength $\sim 3 \times 10^{-8}$ Tesla [215]. More refined calculations replace the rigidity cut-off by a probability distribution 218, 227, 228]. In the rigidity cut-off approximation one can give for every experimental location a contour map depicting curves with constant cut-off in 
a plot with the coordinate axes representing azimuth and zenith angles of the primary cosmic ray particle direction. For Kamioka such a plot is found in Ref. [215]. At the geomagnetic latitude of $90^{\circ}$ the geomagnetic cut-off disappears, therefore experiments situated at high geomagnetic latitudes have a higher flux of low energy atmospheric neutrinos. The magnetic field of the earth is not exactly of dipole form but also has a considerable multipole contribution which affects the local rigidity cut-off maps and thus the atmospheric neutrino flux in particular for down-going neutrinos [215].

Hadronic interactions: After entering the atmosphere the cosmic ray particles collide with the nuclei in the air. Though the fractions of other particles than protons in the cosmic rays are small, their importance for the atmospheric neutrino flux gets enhanced because the predecessors of the neutrinos, pions and kaons, are created through the hadronic interactions of the cosmic rays with the air nuclei. These processes depend rather on the number of nucleons than on the number of nuclei. The ratio of the charged kaon to the charged pion flux depends sensitively on the description of hadronic interactions. In this context the quantities

$$
Z_{p \mathcal{M}} \equiv \int_{0}^{1} \mathrm{~d} x x^{\gamma} \frac{\mathrm{d} N_{p \mathcal{M}}}{\mathrm{d} x},
$$

where $\mathcal{M}=\pi, K$ and $\gamma \simeq 1.7$ comes from the power law of the primary cosmic protons, $x$ is the fraction of the proton momentum carried by the meson and $\mathrm{d} N / \mathrm{d} x$ is the distribution of the charged mesons produced by collisions of protons with nuclei in the atmosphere, are important indicators for the fraction of the muon neutrino flux originating from the $\mathcal{M}$ meson flux. The ratio $Z_{p K^{ \pm}} / Z_{p \pi^{ \pm}}$ranges from 0.10 to 0.15 in different calculations [217]. As a consequence, at neutrino energies below $100 \mathrm{GeV}$ the pions dominate as neutrino sources [216, 221].

Characteristics of the atmospheric neutrino fluxes: Some of the characteristic properties of the atmospheric neutrino fluxes are simple consequences of the production mechanisms (5.1) and (5.2), whereas others follow from a Monte Carlo or analytic calculation 221] of the neutrino fluxes. As mentioned above, the flux calculations are one-dimensional and energy losses in the air, in particular, for the muons, have to be taken into account 219].

a. Approximately, the $\stackrel{(-)}{\nu}$ fluxes have a power law energy spectrum $E_{\nu}^{-3}$ for $1 \lesssim E_{\nu} \lesssim 10^{3}$ $\mathrm{GeV}$ whereas the $\stackrel{(-)}{\nu}$ fluxes decrease like $E_{\nu}^{-3.5}$ [215].

b. Fixing a neutrino energy $E_{\nu}$, the largest contribution to the flux of this energy originates from cosmic ray particles with energy $E_{\text {cr }} \sim 10 \times E_{\nu}$ [215,216].

c. Denoting neutrino fluxes by $(\nu)$ then it follows immediately from Eqs.(5.1) and (5.2) that

$$
\frac{\left(\nu_{\mu}\right)+\left(\bar{\nu}_{\mu}\right)}{\left(\nu_{e}\right)+\left(\bar{\nu}_{e}\right)} \simeq 2 \text {. }
$$


However, it can easily be estimated that, at neutrino energies larger than $1 \mathrm{GeV}$, muons from the reactions (5.1) start to reach the surface of the earth before they decay. Therefore the neutrino fluxes from muon decay decrease, in particular, the $\stackrel{(-)}{\nu_{e}}$ flux, and the ratio (5.5) begins to rise [215, 219].

d. For the same reason muons cease to be the dominant source of $\left(\nu_{e}\right)+\left(\bar{\nu}_{e}\right)$ above $E_{\nu} \simeq 100 \mathrm{GeV}$ and the reactions (5.3) take over [219, 221].

e. Pions cease to be the dominant source of the $\stackrel{(-)}{\nu}$ flux for neutrino energies above 100 $\mathrm{GeV}$ [221]. The reason is given by the different masses and lifetimes of the parent mesons $\mathcal{M}=\pi, K$ in the decays $\mathcal{M} \rightarrow \mu \nu$. For relativistic parent mesons and for a given energy $E_{\nu}$, the lower bound on the energy of the parent meson in such a decay is given by $E_{\mathcal{M}} \geq E_{\nu} /\left(1-m_{\mu}^{2} / m_{\mathcal{M}}^{2}\right)$. Thus, neutrinos with energy $E_{\nu}$ from the decay of the heavier parent meson need in average a lower parent meson energy where the corresponding meson flux is higher. This together with the shorter kaon lifetime leads to the prevailing of kaons as the source of muon neutrinos at sufficiently high neutrino energies [215, 216, 221, 230].

f. The ratio $\left(\bar{\nu}_{e}\right) /\left(\nu_{e}\right)$ is smaller than 1 because among the primary cosmic ray nucleons the protons by far dominate which leads to an enhancement of positively charged pions over negative ones in the hadronic interactions and, consequently, [r $^{-1}\left(\pi^{+}\right) /\left(\pi^{-}\right) \simeq$ $\left(\mu^{+}\right) /\left(\mu^{-}\right)>1$ [215,221].

g. On the other hand, for $E_{\nu} \lesssim 1 \mathrm{GeV}$, where the muons have time to decay in the atmosphere, one has $\left(\bar{\nu}_{\mu}\right) /\left(\nu_{\mu}\right) \simeq 1$ because the muons supply the antineutrinos (neutrinos) to the neutrinos (antineutrinos) from pions and kaons. However, for neutrino energies above a few $\mathrm{GeV}$, when part of the muons reaches the surface of the earth before they decay, this ratio becomes smaller than one because the $\pi^{+}$are more numerous than the $\pi^{-}$(see above).

h. For $E_{\nu} \gtrsim 5 \mathrm{GeV}$ the geomagnetic effects become negligible.

The largest error in the atmospheric neutrino flux calculations arises from an overall uncertainty of primary cosmic ray measurements of the order of $\pm 15 \%$ [215,217]. Therefore, it is mandatory to use quantities which are ratios such that this uncertainty cancels or, in the case of a fit to the data, to include the overall normalization of the primary cosmic ray flux in the set of parameters to be determined by the fit. Among the flux calculations of the different groups the major source of differences comes from the treatment of pion production in collisions of protons with light nuclei [217, 231]. Thus, the calculated absolute fluxes have uncertainties of the order of $\pm 20 \%$ and even larger uncertainties at neutrino energies below $1 \mathrm{GeV}$, whereas flux ratios differ in general only by a few percent (see the comparison between different flux calculations in Refs. [217,231]).

\footnotetext{
${ }^{15}$ In general, we denote particle fluxes by putting the particle symbol within parentheses.
} 
The angular dependence of the atmospheric neutrino flux: The direction of the atmospheric neutrino flux at the location of an experiment is described by the zenith angle $\theta$ and the azimuth angle $\phi$. Neutrinos going vertically downward have $\theta=0$ whereas those coming vertically upward through the earth have $\theta=\pi$. The azimuth angle indicates the direction of the flux in the horizontal plane. There are two natural causes for an angular dependence [218] of the atmospheric neutrino flux:

a. The development of cosmic ray showers in the atmosphere depends on the density of the air. At zenith angles $\theta \sim \pi / 2$, where along the line of flight the increase in density is less steep, pion and kaon decay is enhanced compared to vertical directions. It is easily checked that the situation is symmetric with respect to $\theta \rightarrow \pi-\theta$. Therefore, the generation of pions and kaons in the atmosphere caused by primary cosmic rays induces a dependence of the atmospheric neutrino flux on $|\cos \theta|$. In other words, this mechanism does not introduce an up-down asymmetry in the flux.

b. The geomagnetic field, which acts on the primary cosmic ray flux, is the cause for a $\phi$ and $\theta$ dependence of the atmospheric neutrino flux. Because of the positive charge of the primary cosmic rays the neutrino flux is highest (lowest) for directions coming from the west (east). The nature of the geomagnetic effects also generates an up-down asymmetry for low energy neutrinos [215,218].

A third cause for a dependence on the zenith angle is possibly given by neutrino oscillations which arises because varying $\theta$ from 0 to $\pi$ the neutrino path length varies from around $10 \mathrm{~km}$ 232 to around $13000 \mathrm{~km}$. Clearly, this dependence is not up-down symmetric. If it is disentangled from the up-down asymmetry caused by the geomagnetic effects, e.g., by using the high energy component of the neutrino flux, it provides us with valuable information on neutrino masses and mixing.

\subsubsection{Experiments with atmospheric neutrinos}

Early efforts for detecting atmospheric neutrinos (see Ref. [233] for a summary) concentrated on neutrino-induced upward muons, i.e., muons with zenith angles $90^{\circ} \leq \theta \leq 180^{\circ}$, or horizontal muons, i.e., muons with a zenith angle around $90^{\circ}$, using the process

$$
\stackrel{(-)}{\nu})+N \rightarrow \mu^{ \pm}+X
$$

where $N$ is a nucleon in the rock surrounding the detector located underground [234, 235]. This method was proposed to distinguish muons generated by atmospheric neutrinos from muons originating in the meson decays (5.1) in the atmosphere. There were two experiments, the Kolar Gold Field experiment in India 236] and an experiment in South Africa [237], reporting the first evidence for atmospheric muon neutrinos. Since these experiments could not distinguish up and down directions they used horizontal muons crossing the detector which lay, due to the great depth of the experimental sites, in the large zenith angle intervals $60^{\circ} \leq \theta \leq 120^{\circ}$ and $45^{\circ} \leq \theta \leq 135^{\circ}$ for the Indian and the South African experiment, respectively. These early experiments were accompanied already by atmospheric neutrino flux calculations [230, 238 240]. 
Recent detectors [241] are divided into two classes: water Cherenkov detectors where the neutrino target is a large volume of water surveyed by a huge array of photomultiplier tubes sitting on the surface of the volume (the Kamiokande 449, 242, 243], SuperKamiokande [2] and IMB [50, 244, 245] collaborations) and iron plate calorimeters where neutrino-induced charged particles ionize the gas between the plates and the particle paths are reconstructed electronically (the Fréjus [246], NUSEX [247] and Soudan-2 [51] collaborations). In contrast to the early detectors the recent detectors are sensitive to the direction of tracks and can thus distinguish between up and down through-going tracks. However, they cannot measure the charge of the leptons $\ell^{ \pm}$and thus cannot distinguish between $\nu_{\ell}$ and $\bar{\nu}_{\ell}$. Therefore, at low energies they are approximately sensitive the flux combination $\left(\nu_{\ell}\right)+\frac{1}{3}\left(\bar{\nu}_{\ell}\right)(\ell=e, \mu)$ because at low energies quasi-elastic scattering is predominant and the ratio of quasi-elastic antineutrino to neutrino cross sections is approximately $1 / 3$ 217,233,248. In atmospheric neutrino physics it is important to distinguish $e$-like and $\mu$-like events which is accomplished by distinguishing between showers or diffuse Cherenkov rings $\left(e^{+}, e^{-}, \gamma\right)$ and tracks or sharp Cherenkov rings $\left(\mu^{+}, \mu^{-}\right.$and charged pions, kaons, protons etc.). The separation between $e$-like and $\mu$-like events is very good, e.g., for Super-Kamiokande its efficiency is estimated to be $98 \%$ or better [2].

For the deep underground detectors two different event classes are defined [241]. Events in which the neutrino interacts with the material inside the detector and where all particles from the neutrino interaction deposit their energies inside the detector are called contained events. The second class refers to events where muon neutrinos interact with the material surrounding the detector via charged current interactions such that the high energy muons enter the detector [249]. In this way one distinguishes through-going muons and stopping muons. Recent measurements of the upward muon flux were performed by the Baksan [250], Kamiokande [251,252], IMB [50], Fréjus [246], MACRO [52] and Super-Kamiokande [1] collaborations.

Super-Kamiokande - and before also Kamiokande - has an inner detector surrounded by an outer detector. This allows to further subdivided the contained events into fully contained events (FC) with all energy of an event deposited in the inner detector and partially contained events (PC) which have exiting tracks detected also in the outer detector. In Kamiokande and Super-Kamiokande, FC events are separated into those having a visible energy $E_{\text {vis }}<1.33 \mathrm{GeV}$, the sub-GeV events, and those with $E_{\text {vis }}>1.33$ $\mathrm{GeV}$, the multi-GeV events. Among the contained events in Cherenkov detectors the single-ring events are well understood, they are predominantly produced by quasi-elastic scattering of electron and muon neutrinos. In Kamiokande and Super-Kamiokande for the analyses of FC events only single-ring events are used with the additional criteria $p_{e} \geq 100 \mathrm{MeV}$ and $p_{\mu} \geq 200 \mathrm{MeV}$ for electron and muon momenta, respectively, in the case of sub-GeV events. To quote the numbers of Super-Kamiokande [2], a Monte Carlo simulation has shown that $88 \%(96 \%)$ of the sub-GeV e-like ( $\mu$-like) events are charged current interactions whereas for multi-GeV events the number is $84 \%$ (99\%). The remainder is given by neutral current events. The PC events were estimated to be $98 \% \stackrel{(-)}{\nu}$-induced events for single and multi-ring configurations and, therefore, all PC events are used for the analyses. 
For the analyses of atmospheric neutrino data it is very important to have a good understanding of the neutrino interactions in the detector. The deep-inelastic scattering (DIS) formulas are only valid for sufficiently high momentum transfer $Q^{2}$ from the leptons to the hadrons. For neutrinos with a few $\mathrm{GeV}$ interacting with the detector material, the lowest multiplicity exclusive channels represent an important fraction of the cross section. Therefore the following decomposition of the neutrino cross section [253 has been proposed:

$$
\sigma_{\nu}^{\mathrm{CC}}=\sigma_{\mathrm{QEL}}+\sigma_{1 \pi}+\sigma_{\mathrm{DIS}},
$$

where QEL indicates the quasi-elastic $\nu_{\ell}+n \rightarrow \ell^{-}+p$ and $\bar{\nu}_{\ell}+p \rightarrow \ell^{+}+n$ cross sections and $1 \pi$ single pion production. In the case of the latter it is assumed that $\Delta(1232)$ dominates. To avoid double counting in $\sigma_{1 \pi}+\sigma_{\text {DIS }}$, the maximal mass of the pion nucleon system in the final state is taken to be $W_{c}=1.4 \mathrm{GeV}$ for single pion production whereas the kinematical region for DIS is bounded by $W \geq W_{c}$, where $W$ is the mass of the hadronic final state.

\subsubsection{The atmospheric neutrino anomaly}

The first observable to be measured in recent atmospheric neutrino experiments was the ratio of $\mu$-like to $e$-like events denoted by $(\mu / e)_{\text {data. }}$. As discussed earlier, in flux ratios the large uncertainty in the overall normalization of the primary cosmic ray flux cancels and there is also some cancellation of errors in the theoretical calculation. However, the above ratio is only a limited reflection of the corresponding ratio of atmospheric neutrino fluxes because of detector efficiencies and event selection criteria. Thus for the expected ratio one has to fold the theoretical flux calculations with the cross sections of the neutrino interactions in the detector and the detection efficiencies and apply the event selection criteria. Quoting the result of the Monte Carlo calculation of Super-Kamiokande as an example, this collaboration obtains $(\mu / e)_{\mathrm{MC}}=1.50$ and 2.83 for the sub-GeV ratio of FC events and the ratio considering multi-GeV FC and PC events, respectively [2], using the neutrino flux calculations of Ref. [215]. Note that these numbers significantly deviate from the naive expectation 2 . Therefore, the actual physically relevant quantity is given by the double ratio

$$
R=\frac{(\mu / e)_{\mathrm{data}}}{(\mu / e)_{\mathrm{MC}}} .
$$

The first indication that this ratio is smaller than 1 was reported more than ten years ago [254]. In the meantime the most impressive measurements of $R$ are represented by the Kamiokande and Super-Kamiokande results:[ద]

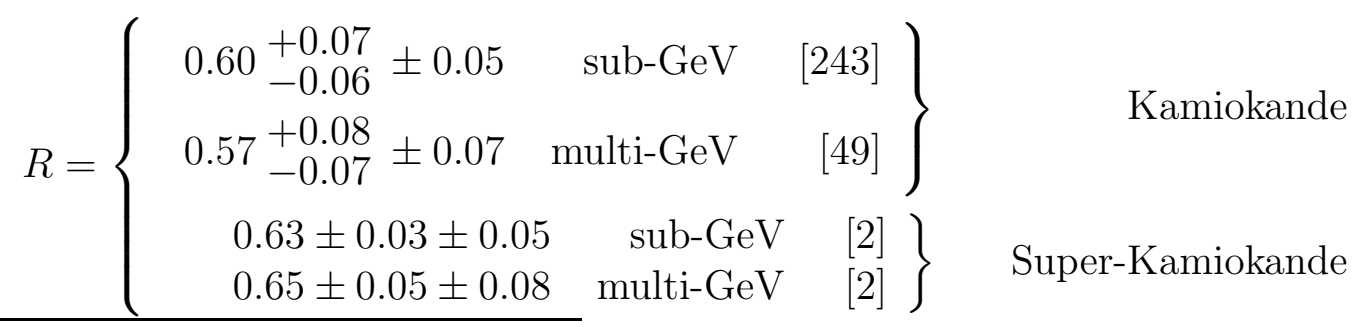

\footnotetext{
${ }^{16}$ For recent results on atmospheric neutrinos see Note added.
} 
The PC events have been added to the multi-GeV data. The results $R=0.54 \pm 0.05 \pm$ 0.11 of the IMB Collaboration [50, 255] and $R=0.61 \pm 0.15 \pm 0.05$ of Soudan-2 256] are in agreement with those of the experiments in Kamioka. However, the early iron calorimeter experiments found values of $R$ compatible with 1 , namely $R=1.00 \pm 0.15 \pm$ 0.08 (Fréjus Coll. 246) and $R=0.96_{-0.28}^{+0.32}$ (NUSEX Coll. 247]). Apart from the latter two experiments, all others hint at a reduction of $R$ compared to the expectation $R=1$.

Kamiokande and, in particular, Super-Kamiokande have enough statistics to study the zenith angle dependence of the measured $\stackrel{(-)}{\nu}$ and $\stackrel{(-)}{\nu}$ fluxes. To this end, the $\cos \theta$ interval $[-1,1]$ is divided into five bins of length 0.4 . Kamiokande 49 has observed a zenith angle variation of $R$ for the $\mathrm{FC}$ multi-GeV $+\mathrm{PC}$ events with indications that the zenith angle variation rather comes from $\mu$-like events than from the $e$-like events. All this is amply confirmed by Super-Kamiokande with much more statistics and with a significant zenith angle variation in the $\mu$-like events for both, sub-GeV and multi-GeV.

It is important to study the zenith angle variation independent of the double ratio $R$ in order to disentangle the zenith angle dependencies of the electron and muon neutrino fluxes. To this end, in addition to the oscillation parameters, also the normalization of primary cosmic ray flux has to be fitted. Super-Kamiokande has performed a statistical analysis of the data under the assumption of $\stackrel{(-)}{\nu} \rightarrow \stackrel{(-)}{\nu}$ oscillations. With $e$-like and $\mu$ like events in five $\cos \theta$ bins and seven momentum bins there are altogether 70 data points and three quantities to be determined: the mixing angle $\vartheta$, the neutrino masssquared difference $\Delta m^{2}$ and the overall neutrino flux normalization. The best fit gives $\sin ^{2} 2 \vartheta=1$ and $\Delta m^{2}=2.2 \times 10^{-3} \mathrm{eV}^{2}$ with $\chi_{\text {min }}^{2}=65.2$ for 67 DOF. The regions in the $\sin ^{2} 2 \vartheta-\Delta m^{2}$ plane allowed at $68 \%, 90 \%$ and $99 \%$ confidence level in the case of $\stackrel{(-)}{\nu_{\mu}} \rightarrow \stackrel{(-)}{\nu_{\tau}}$ oscillations are shown in Fig. 5.1 [2]. At 90\% CL the mass-squared difference lies in the interval $5 \times 10^{-4} \mathrm{eV}^{2}<\Delta m^{2}<6 \times 10^{-3} \mathrm{eV}^{2}$. For the simulation of neutrino oscillations the profiles for the neutrino production heights of Ref. 232 were used. An analogous procedure with the hypothesis of $\stackrel{(-)}{\nu} \leftrightarrow \stackrel{(-)}{\nu})_{e}$ oscillations, taking into account matter effects in the earth, gives a poor fit with $\chi_{\min }^{2}=87.8$ for $67 \mathrm{DOF}$.

It is interesting to note that, as shown in Fig. 5.1, a fit for Kamiokande with $\stackrel{(-)}{\nu_{\mu}} \rightarrow \stackrel{(-)}{\nu_{\tau}}$ oscillations gives $5 \times 10^{-3} \mathrm{eV}^{2}<\Delta m^{2}<3 \times 10^{-2} \mathrm{eV}^{2}$ with $90 \%$ CL 49 for the multi-GeV data whereas the sub-GeV data show no indication for a zenith angle dependence of the number of events. However, Kamiokande has a lower statistics than Super-Kamiokande. In Refs. 257 261 analyses of all available experiments were performed using the three possible oscillation hypotheses $\stackrel{(-)}{\nu} \leftrightarrow \stackrel{(-)}{\nu} \nu_{e, \tau, s}$ with similar results as described above and showing in addition that also the hypothesis of oscillations into sterile neutrinos gives a satisfactory fit. For analyses with three neutrinos and including all neutrino oscillation experiments see 261 266]. In Ref. [245] (IMB) no zenith angle variation of $R$ was seen though with rather small statistics.

In the Super-Kamiokande experiment a significant up-down asymmetry for the $\mu$-like events was found. The measured value of the asymmetry $(U-D) /(U+D)$ as a function of momentum for $e$-like and $\mu$-like events is shown in Fig. 5.2 [2]. Here $U$ is the number 


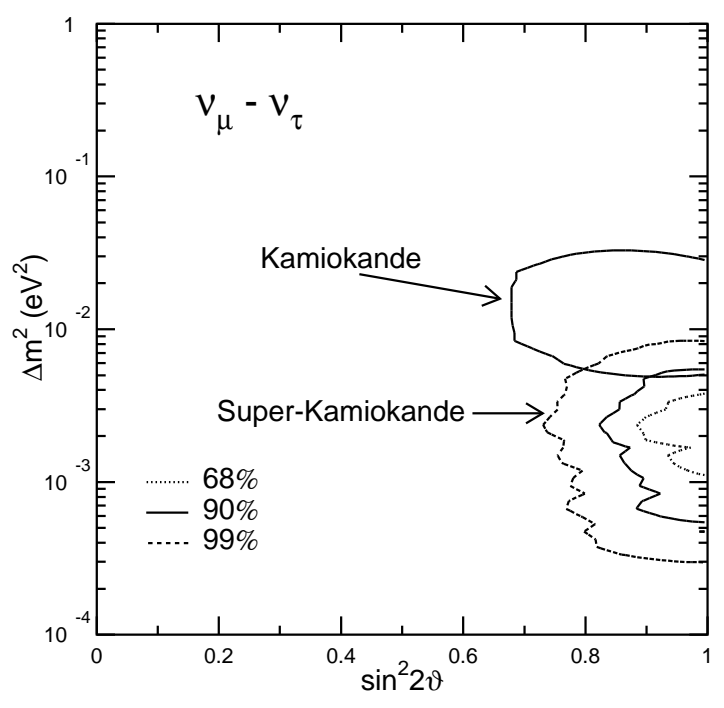

Figure 5.1. The $68 \%, 90 \%$ and $99 \%$ confidence regions in the $\sin ^{2} 2 \vartheta-\Delta m^{2}$ plane for $\nu_{\mu} \rightarrow \nu_{\tau}$ oscillations obtained from 33.0 kton year of SuperKamiokande data [2]. The $90 \%$ confidence region obtained by the Kamiokande experiment is also shown.

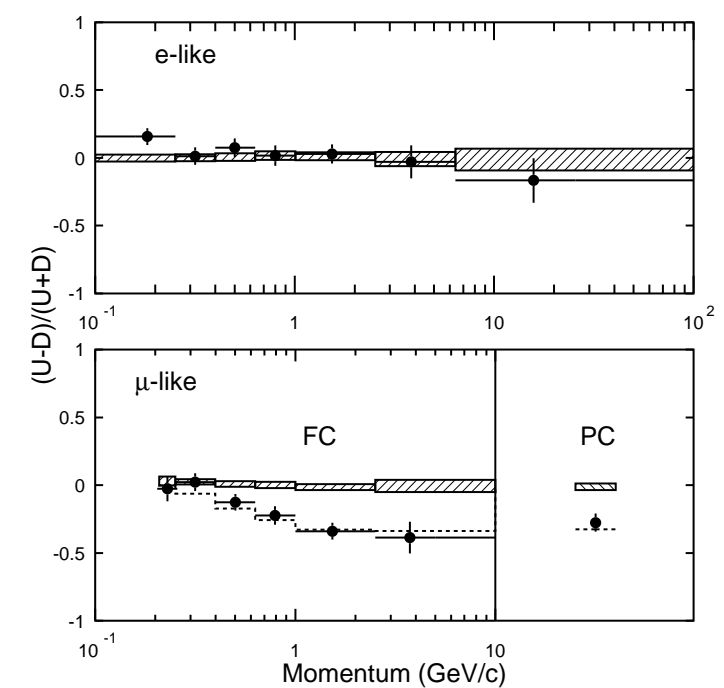

Figure 5.2. Up-down asymmetry measured in the Super-Kamiokande atmospheric neutrino experiment as a function of the momentum of $e$-like and $\mu$-like events [2]. The hatched region shows the theoretical expectation without neutrino oscillations, with statistical and systematic errors added in quadrature. The dashed line for $\mu$ like events is the theoretical expectation in the case of two-generation $\nu_{\mu} \rightarrow \nu_{\tau}$ oscillations with $\Delta m^{2}=2.2 \times 10^{-3} \mathrm{eV}^{2}$ and $\sin ^{2} 2 \vartheta=1.0$.

of upward-going events with zenith angles in the range $-1<\cos \theta<-0.2$ and $D$ the number of downward-going events with $0.2<\cos \theta<1$. The value of the asymmetry for $\mathrm{FC}$ and $\mathrm{PC}$ multi-GeV $\mu$-like events is [2]

$$
A_{\mu} \equiv\left(\frac{U-D}{U+D}\right)_{\mu}=-0.296 \pm 0.048 \pm 0.01
$$

The geomagnetic effect which is one of the causes of an up-down asymmetry contributes less than \pm 0.01 for multi-GeV events [2]. For the $\mathrm{PC}$ events with a mean neutrino energy of $15 \mathrm{GeV}$ [2] this effect is even less important. Note that for multi-GeV events the average angle between the incoming neutrino direction and the charged lepton seen in the single-ring events is of the order of $20^{\circ}$ or less. Since $\cos \theta= \pm 0.2$ corresponds to $90^{\circ} \pm 11.5^{\circ}$, the asymmetry $A$ of the $\mu$-like events is in effect an up-down asymmetry of the $\stackrel{(-)}{\nu}_{\mu}$ flux. The result $(5.10)$ constitutes the best indication in favour of neutrino oscillations found so far with $A_{\mu}$ being 6 standard deviations away from 0 . This is corroborated by the plots of $A_{e, \mu}(p)$ as a function of the lepton momentum $p$ in the case of the single-ring events shown in Fig. 5.2 [2]. For $e$-like events, $A_{e}(p)$ is a flat function consistent with no $\stackrel{(-)}{\nu} \leftrightarrow \stackrel{(-)}{\nu_{\mu}}$ oscillations. The asymmetry for $e$-like events analogous to Eq.(5.10) is compatible with zero: $A_{e}=-0.036 \pm 0.067 \pm 0.02$. For the $\mu$-like events, $A_{\mu}(p)$ starts with zero and decreases, thus indicating that at small energies both up and down-going neutrinos oscillate with averaged $\stackrel{(-)}{\nu} \rightarrow \stackrel{(-)}{\nu}$ probabilities $1 / 2$ whereas at muon 
energies in the GeV range the probability of $\stackrel{(-)}{\nu} \rightarrow \stackrel{(-)}{\nu}$ survival for the down-going neutrinos approaches 1 . This behaviour is in agreement with the $L / E_{\nu}$ dependence of the oscillation probabilities. It is interesting to note that the experimental values of the asymmetries $A_{e, \mu}$ can be used to discriminate between different oscillation scenarios 267 270].

The importance of the results of the atmospheric neutrino oscillation experiments requires further scrutiny to test the interpretation in terms of neutrino oscillations. It has been proposed for Super-Kamiokande to use ratios of charged current events (CC) to neutral current (NC) events [271,272] in the spirit of the SNO experiment 273] in the context of solar neutrinos. The basic idea is that in Super-Kamiokande NC events could be seen through

$$
\stackrel{(-)}{\nu_{\ell}}+N \rightarrow \stackrel{(-)}{\nu}{ }_{\ell}+\pi^{0}+N \quad \text { with } \quad N=p, n \quad \ell=e, \mu, \tau
$$

whereas CC reactions are likely to produce a single charged pion via

$$
\stackrel{(-)}{\nu})+N \rightarrow \ell^{\mp}+\pi^{ \pm}+N \quad \text { with } \quad \ell=e, \mu .
$$

To produce a test with the reactions (5.11) and (5.12) it is necessary to discuss their experimental signatures and the contaminations of these signature with other processes. Neutral pions are selected by taking events with two diffuse rings from the two decay photons with an invariant mass between 90 and $180 \mathrm{MeV}$ and momenta smaller $400 \mathrm{MeV}$ in order to separate the two rings. Such events were already observed in Kamiokande [274. Because of the selection criteria for $\pi^{0}$ 's, single pion production is important only for neutrino energies of order $1 \mathrm{GeV}$, therefore it involves the sub-GeV events and no tau lepton will produced in the CC reaction. Defining $N_{\pi^{0}}$ via this procedure then the two-ring ratios 272

$$
\mathcal{R}_{e} \equiv \frac{N_{D S}}{N_{\pi^{0}}} \quad \text { and } \quad \mathcal{R}_{\mu} \equiv \frac{N_{S S}}{N_{\pi^{0}}}
$$

act as measures of the $\mathrm{CC}$ to $\mathrm{NC}$ event ratios because in the ideal situation one can make the identification $N_{\pi^{0}}=N_{\pi^{0}}^{N C}, N_{D S}=N_{e^{\mp} \pi^{ \pm}}^{C C}$ and $N_{S S}=N_{\mu^{\mp} \pi^{ \pm}}^{C C}$ since electrons produce diffuse and muons and charged pions sharp rings. This picture is blurred [272, 275] by detector efficiencies (e.g., it is 0.77 for the identification of neutral pions via two diffuse rings), misidentifications (like misidentifying a $\pi^{0}$ whose two photons cannot be resolved with an electron) and contaminations (most notably, NC events with $\pi^{+} \pi^{-}$in the final state contribute to $N_{S S}$, electronic CC events with a $\pi^{0}$ whose photons cannot be resolved add to $N_{\pi^{0}}$ and muonic CC events with the same $\pi^{0}$ configuration add to $\left.N_{D S}\right)$. Taking all this into account [272, 275], one has nevertheless reason the expect that with increasing statistics in Super-Kamiokande the two-ring events can be used to obtain information on distinguishing between oscillation of the muon neutrino into tau or sterile neutrinos and to discriminate between different regions of the parameter space of neutrino mixing [272, 275, 276]. Furthermore, it was suggested to use the asymmetry $A_{N} \equiv\left(U_{\pi^{0}}-D_{\pi^{0}}\right) /\left(U_{\pi^{0}}+D_{\pi^{0}}\right)$ for up and down-going $\pi^{0}$ 's coming from the NC reaction (5.11) as an observable to distinguish muon neutrino oscillations into active neutrinos from those into sterile neutrinos [277]. One has to assume in this case that up and down 
$\pi^{0}$ 's originate from up and down neutrinos, respectively, with high probability. This is not so obvious, however, since for the identification of the $\pi^{0}$ the events should rather have low energy as explained above.

The further tests discussed here concern the stopping and through-going muon events where special efforts have been made to calculate the fluxes [249, 253, 278]. Whereas the FC events have neutrino energies of around $1 \mathrm{GeV}$ the stopping muon events correspond to a mean neutrino energy of $10 \mathrm{GeV}$ and the through-going muons to $100 \mathrm{GeV}$ [231,249]. Thus we are now discussing a different range of energy compared to the discussion above (with the exception of the PC sample).

Obviously, also the zenith angle distribution of upward stopping or through-going muons can be used to test the neutrino oscillation hypothesis 279. Among other experiments (see above) upward through-going muons have been studied by Kamiokande 252] and upward muons by MACRO [52]. Kamiokande has 372 such events above an energy threshold of $1.6 \mathrm{GeV}$. Fitting the data to the $\left.\stackrel{(-)}{\nu_{\mu}} \rightarrow \stackrel{(-)}{\nu}\right)_{\tau}$ oscillation hypothesis yields a best fit with $\Delta m^{2}=3.2 \times 10^{-3} \mathrm{eV}^{2}$ agreeing rather well with the Super-Kamiokande result. The analysis of the MACRO Coll. based on 479 events gives a similar result for $\Delta m^{2}$, however, the zenith angle distribution does not fit very well with the oscillation hypothesis into tau neutrinos. An attempt has been made to explain the zenith angle distribution of the MACRO experiment with $\stackrel{(-)}{\nu_{\mu}} \rightarrow \stackrel{(-)}{\nu} \nu_{s}$ oscillations where matter effects in the earth play a crucial role 280.

In the earth, the density profile can approximately be represented by constant densities in the mantle and the core, respectively (see end of Section 4.3). Such a profile can lead to an enhancement of neutrino transitions due to the matter effect $\square$ in the earth if atmospheric neutrinos cross the core ${ }^{\text {to }}$ such that the phase picked up by a neutrino wave function traversing the mantle for the first time and the phase acquired by traversing the core are each approximately equal to $\pi$. Such an effect for atmospheric neutrinos was recently considered in detail in Refs. [280,282 285]. It has also been proposed to exploit this effect to discriminate between $\stackrel{(-)}{\nu} \underset{\mu}{\rightarrow} \stackrel{(-)}{\nu}$ (no matter effects) and $\stackrel{(-)}{\nu} \rightarrow \stackrel{(-)}{\nu}$ transitions of atmospheric neutrinos [280] and to explain an excess of $e$-like events [286] possibly seen in the Super-Kamiokande experiment [2].

It was suggested in Ref. 244 to use the observable

$$
r=\frac{N_{\text {stop }}}{N_{\text {thru }}}
$$

where $N_{\text {stop }}$ and $N_{\text {thru }}$ are the numbers of stopping and though-going muons, respectively, as an indicator for neutrino oscillations because this ratio is reduced for neutrino oscillations with respect to the no-oscillation hypothesis [253, 287]. Also the ratios of "horizontal" to "vertical" muon events for stopping and through-going muons defined by

$$
\left.S_{\text {stop,thru }} \equiv \frac{N_{\text {hor }}}{N_{\text {vert }}}\right|_{\text {stop,thru }},
$$

\footnotetext{
${ }^{17}$ Note that for the case of a periodical matter density the effect of enhancement was considered in Refs. [281].

${ }^{18}$ Neutrinos with $\cos \theta \leq-0.837$
} 
with

$$
N_{\text {hor }}=\int_{\cos \theta_{c}}^{0} d \cos \theta \frac{d N}{d \cos \theta} \quad \text { and } \quad N_{\text {vert }}=\int_{-1}^{\cos \theta_{c}} d \cos \theta \frac{d N}{d \cos \theta}
$$

are useful observables as shown in Ref. 287]. In this work a study was made for SuperKamiokande, taking $\cos \theta_{c}=-0.5$ as the boundary between "horizontal" and "vertical", showing that the prospects are good for confirming atmospheric neutrino oscillations found with the FC single-ring and PC events by using stopping and through-going muons and the observables $r$ (5.14), $S_{\text {stop }}$ and $S_{\text {thru }}$ (5.15). However, for a precise determination of $\Delta m^{2}$ these variables are not suitable.

\subsubsection{Long-baseline experiments and tests of the atmospheric neutrino os- cillation parameters}

The explanation of the atmospheric neutrino anomaly in terms of neutrino oscillations can be checked with long-baseline neutrino oscillation experiments. The first long-baseline reactor experiment $\mathrm{CHOOZ} \mathrm{[159]} \mathrm{(see} \mathrm{Section} 3.2$ and Fig. 3.3) has already excluded atmospheric $\nu_{\mu} \leftrightarrows \nu_{e}$ oscillations with a large mixing angle for $\Delta m_{\mathrm{atm}}^{2} \gtrsim 10^{-3} \mathrm{eV}^{2}$. Two other long-baseline reactor experiments are under construction: Palo Verde 288, 289] and Kam-Land [290, 291]. The Palo Verde experiment has a setup and a sensitivity similar to the CHOOZ experiment, whereas the Kam-Land experiment, which is the result of the conversion of the old Kamiokande detector to a liquid scintillator detector, will detect $\bar{\nu}_{e}$ 's produced by Japanese reactors $150-200 \mathrm{~km}$ away and will be sensitive to $\Delta m^{2} \gtrsim 10^{-5} \mathrm{eV}^{2}$ and a large mixing angle. The Borexino experiment (see the end of the Section 5.2 and [291,292]) will allow to perform a similar measurement.

Accelerator long-baseline experiments will study the oscillation channels $\nu_{\mu} \rightarrow \nu_{e, \mu, \tau}$. The K2K 271 experiment, with a baseline of about $235 \mathrm{~km}$ from KEK to SuperKamiokande and a neutrino beam with $1.4 \mathrm{GeV}$ average energy, will be sensitive to $\nu_{\mu}$ disappearance and $\nu_{\mu} \rightarrow \nu_{e}$ transitions with $\Delta m^{2} \gtrsim 2 \times 10^{-3} \mathrm{eV}^{2}$. A near 1 kton waterCherenkov detector will be placed at a distance of about $1 \mathrm{~km}$ from the beam dump and will allow to measure the initial flux and energy spectrum of $\nu_{\mu}$ 's. This experiment is under construction and is planned to begin taking data in the year 1999 .

Also the MINOS [293] experiment is under construction. This experiment will have a near detector at Fermilab and a baseline of about $730 \mathrm{~km}$ from Fermilab to the Soudan mine, where the far detector will be placed. The neutrino beam will be produced by protons from the new Main Injector at Fermilab and will have an average energy of about $10 \mathrm{GeV}$. The far detector is an 8 kton sampling calorimeter made of magnetized iron and scintillators. This experiment will be sensitive to $\nu_{\mu}$ disappearance and $\nu_{\mu} \rightarrow \nu_{e}$, $\nu_{\mu} \rightarrow \nu_{\tau}, \nu_{\mu} \rightarrow \nu_{s}$ transitions, with the possibility to distinguish the different channels, for $\Delta m^{2} \gtrsim 10^{-3} \mathrm{eV}^{2}$ (the possibility to extend the sensitivity to $\Delta m^{2} \gtrsim 5 \times 10^{-5} \mathrm{eV}^{2}$ lowering the neutrino energy is under study). In particular $\nu_{\mu} \rightarrow \nu_{s}$ transitions can be revealed through the measurement of a deficit in the $\mathrm{NC} / \mathrm{CC}$ ratio. The MINOS experiment is scheduled to start data-taking around the year 2003.

The ICARUS experiment 294] in Gran Sasso, constituted of a 0.6 kton liquid argon detector is scheduled to start in the year 2000. In the future three new modules with a 
total mass of 2.4 kton will be installed. This detector will be sensitive to atmospheric and solar neutrinos and will allow to reveal long-baseline $\nu_{\mu} \rightarrow \nu_{\tau}$ oscillations using a neutrino beam produced at CERN about $730 \mathrm{~km}$ away. Since the average energy of the neutrino beam is rather high, about $25 \mathrm{GeV}$, in order to allow the detection of $\nu_{\tau}$ through the $\mathrm{CC}$ production of a $\tau$, this experiment will be sensitive to $\Delta m^{2} \gtrsim 10^{-3} \mathrm{eV}^{2}$. Four other detectors for future LBL CERN-Gran Sasso experiments, OPERA [295], NOE 296], AQUA-RICH [297] and NICE [298], have been proposed and are under consideration (see Ref. 299]), together with the possibility of a new atmospheric neutrino detector consisting of a large-mass and high-density tracking calorimeter [300].

In Ref. [301] a comparison is made between the possibilities using atmospheric neutrinos and LBL neutrino experiments for the determination of the oscillation parameters. The feasibility to distinguish between atmospheric $\nu_{\mu} \rightarrow \nu_{\tau}$ and $\nu_{\mu} \rightarrow \nu_{s}$ oscillations using a combination of the results of future atmospheric, LBL and SBL experiments is discussed in Ref. 302.

\subsection{Solar neutrino experiments}

The earliest indication in favour of neutrino oscillations was obtained about 30 years ago in the radiochemical solar neutrino experiment by $\mathrm{R}$. Davies et al. [36]. The flux of solar electron neutrinos measured in this experiment was significantly less than the predicted one. This phenomenon was called solar neutrino problem. The existence of this problem was confirmed in all five solar neutrino experiments (Homestake 36 38], Kamiokande [39 41, GALLEX [42,43], SAGE [44, 45] and Super-Kamiokande 46 48]) which measure a flux of electron neutrinos significantly smaller than the one predicted by the Standard Solar Model (SSM) 60, 174, 175, 303 310. The solar neutrino problem (see, for example, [60, 311 [316]) arose in the Homestake experiment by the low counting rate showing that the flux of the high-energy ${ }^{8} \mathrm{~B}$ neutrinos $\left(E_{8_{\mathrm{B}}} \lesssim 15 \mathrm{MeV}\right)$ and of the mediumenergy ${ }^{7} \mathrm{Be}$ neutrinos $\left(E_{7 \mathrm{Be}}=0.862 \mathrm{MeV}\right)$ is suppressed by a factor of about $1 / 3$ with respect to the SSM prediction. In 1988 the solar neutrino problem was confirmed by the results of the real-time water-Cherenkov Kamiokande experiment [39] which measured a flux of ${ }^{8} \mathrm{~B}$ neutrinos of about half of the SSM flux. The measurements of the Kamiokande experiment proved that the observed neutrinos arrive at the detector from the direction of the sun. In 1992 the radiochemical GALLEX [42] and SAGE experiments [45] succeeded in measuring the neutrino flux with a low energy threshold $E_{\mathrm{th}}=233 \mathrm{keV}$, which allowed to detect low-energy $p p$ neutrinos produced by the fundamental reaction of the $p p$ cycle. Also these experiments measured a neutrino flux of about half of the one predicted by the SSM. Finally, the Super-Kamiokande experiment has recently confirmed 46 48 with high statistics the suppression of the ${ }^{8} \mathrm{~B}$ neutrino flux with respect to the SSM one by a factor of about $1 / 2$.

The energy of the sun is produced in the reactions of the thermonuclear $p p$ and CNO cycles shown in Figs. 5.3 and 5.4 (see, e.g., Ref. [60]). The overall result of both cycles is the transition

$$
4 p+2 e^{-} \rightarrow{ }^{4} \mathrm{He}+2 \nu_{e}+Q
$$




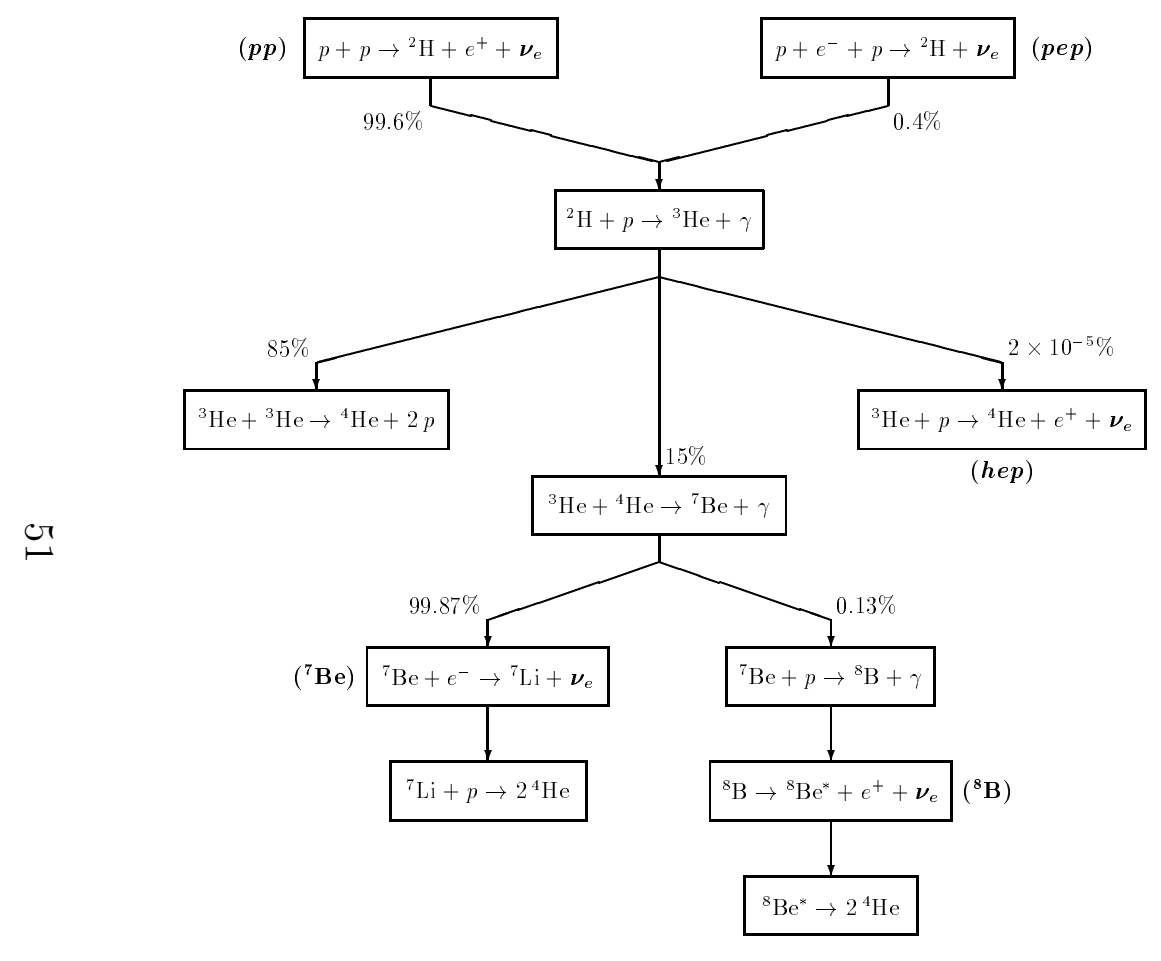

Figure 5.3. The $p p$ cycle.

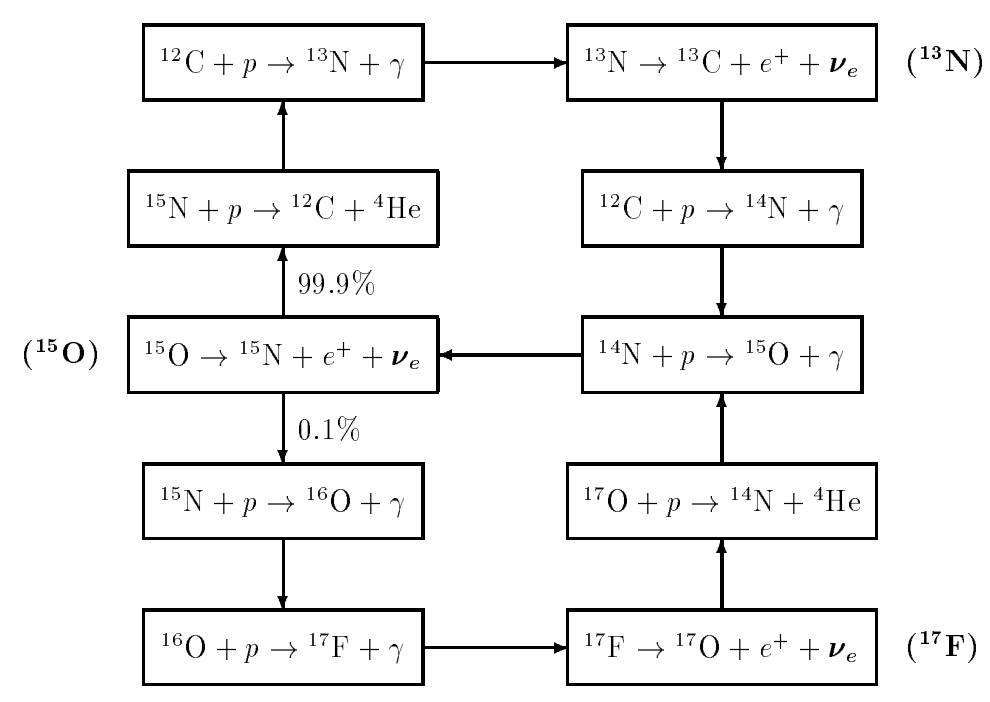

Figure 5.4. The CNO cycle. 


\begin{tabular}{cccc} 
Source $r$ & Reaction & $\begin{array}{c}\text { Average Neutrino } \\
\text { Energy }\langle E\rangle_{r}(\mathrm{MeV})\end{array}$ & $\begin{array}{c}\text { Maximum Neutrino } \\
\text { Energy }(\mathrm{MeV})\end{array}$ \\
\hline \hline$p p$ & $p+p \rightarrow d+e^{+}+\nu_{e}$ & 0.2668 & $0.423 \pm 0.03$ \\
\hline$p e p$ & $p+e^{-}+p \rightarrow d+\nu_{e}$ & 1.445 & 1.445 \\
\hline${ }^{7} \mathrm{Be}$ & $e^{-}+{ }^{7} \mathrm{Be} \rightarrow{ }^{7} \mathrm{Li}+\nu_{e}$ & 0.3855 & 0.3855 \\
\hline${ }^{8} \mathrm{~B}$ & ${ }^{8} \mathrm{~B} \rightarrow{ }^{8} \mathrm{Be}^{*}+e^{+}+\nu_{e}$ & $6.735 \pm 0.036$ & 0.8631 \\
\hline$h e p$ & ${ }^{3} \mathrm{He}+p \rightarrow{ }^{4} \mathrm{He}+e^{+}+\nu_{e}$ & 9.628 & 18.778 \\
\hline${ }^{13} \mathrm{~N}$ & ${ }^{13} \mathrm{~N} \rightarrow{ }^{13} \mathrm{C}+e^{+}+\nu_{e}$ & 0.7063 & $1.1982 \pm 0.0003$ \\
\hline${ }^{15} \mathrm{O}$ & ${ }^{15} \mathrm{O} \rightarrow{ }^{15} \mathrm{~N}+e^{+}+\nu_{e}$ & 0.9964 & $1.7317 \pm 0.0005$ \\
\hline${ }^{17} \mathrm{~F}$ & ${ }^{17} \mathrm{~F} \rightarrow{ }^{17} \mathrm{O}+e^{+}+\nu_{e}$ & 0.9977 & $1.7364 \pm 0.0003$ \\
\hline \hline
\end{tabular}

Table 5.1. Sources of solar neutrinos 317 319.

where $Q=4 m_{p}+2 m_{e}-m_{4} \mathrm{He}=26.73 \mathrm{MeV}$ is the energy release. Hence, the production of energy in the sun is accompanied by the emission of electron neutrinos. The main part of the solar energy is radiated through photons and a small part (about 2\%) is emitted through neutrinos. The sources of solar neutrinos are listed in Table 5.1. The pp, pep, ${ }^{7} \mathrm{Be},{ }^{8} \mathrm{~B}$ and hep reactions belong to the $p p$ cycle (see Fig. 5.3), whereas the ${ }^{13} \mathrm{~N},{ }^{15} \mathrm{O}$ and ${ }^{17} \mathrm{~F}$ reactions belong to the CNO cycle (see Fig. 5.4), which produces only about $2 \%$ of the solar energy. The average and maximum neutrino energies listed in Table 5.1 are taken from Refs. 317, 318. The neutrino fluxes and predictions for the neutrino capture rates in the chlorine Homestake experiment and in the gallium GALLEX and SAGE experiments given by the Bahcall-Pinsonneault 1998 (BP98) [304] SSM are listed in Table 5.2.

As it is seen from the Tables 5.1 and 5.2, the major part of solar neutrinos are low energy neutrinos coming from the $p p$ reaction. Monoenergetic neutrinos with intermediate energy are produced in the capture of electrons by ${ }^{7} \mathrm{Be}$ and in the pep reaction. High energy neutrinos are produced in the decay of ${ }^{8} \mathrm{~B}$ (the flux of hep neutrinos is so small that its contribution to the event rates of solar neutrino experiments is negligible). The flux of ${ }^{8} \mathrm{~B}$ neutrinos is much smaller than the fluxes of $p p,{ }^{7} \mathrm{Be}$ and $p e p$ neutrinos. However, as we will see later, these neutrinos give the major contribution to the event rates of experiments with a high energy detection threshold. The $\mathrm{CNO}{ }^{13} \mathrm{~N},{ }^{15} \mathrm{O},{ }^{17} \mathrm{~F}$ reactions are sources of intermediate energy neutrinos with a spectrum that extends up to about 1.7 MeV. Their contribution to the event rates of solar neutrino experiment is small but not negligible.

\footnotetext{
${ }^{19}$ Here $m_{p}=938.272 \mathrm{MeV}$ is the proton mass, $m_{e}=0.511 \mathrm{MeV}$ is the electron mass and $m_{4} \mathrm{He}=$ $2 m_{p}+2 m_{n}-E_{4 \mathrm{He}}^{B}$ is the mass of the ${ }^{4} \mathrm{He}$-nucleus, where $m_{n}=939.566 \mathrm{MeV}$ is the neutron mass and $E_{4 \mathrm{He}}^{B}=28.296 \mathrm{MeV}$ is the binding energy of the ${ }^{4} \mathrm{He}-$ nucleus.
} 


\begin{tabular}{cccccc} 
Source $r$ & $\begin{array}{c}\text { Flux } \Phi_{r} \\
\left(\mathrm{~cm}^{-2} \mathrm{~s}^{-1}\right)\end{array}$ & $\begin{array}{c}\left\langle\sigma_{\mathrm{Cl}}\right\rangle_{r} \\
\left(10^{-44} \mathrm{~cm}^{2}\right)\end{array}$ & $\begin{array}{c}S_{\mathrm{Cl}}^{(r)} \\
(\mathrm{SNU})\end{array}$ & $\begin{array}{c}\left\langle\sigma_{\mathrm{Ga}}\right\rangle_{r} \\
\left(10^{-44} \mathrm{~cm}^{2}\right)\end{array}$ & $\begin{array}{c}S_{\mathrm{Ga}}^{(r)} \\
(\mathrm{SNU})\end{array}$ \\
\hline \hline$p p$ & $(5.94 \pm 0.06) \times 10^{10}$ & - & - & $0.117 \pm 0.003$ & $69.6 \pm 0.7$ \\
\hline$p e p$ & $(1.39 \pm 0.01) \times 10^{8}$ & 0.16 & 0.2 & $2.04_{-0.14}^{+0.35}$ & 2.8 \\
\hline${ }^{7} \mathrm{Be}$ & $(4.80 \pm 0.43) \times 10^{9}$ & 0.024 & $1.15 \pm 0.1$ & $0.717_{-0.0 .021}^{+0.050}$ & $34.4 \pm 3.1$ \\
\hline${ }^{8} \mathrm{~B}$ & $\left(5.15_{-0.72}^{+0.98}\right) \times 10^{6}$ & $114 \pm 11$ & $5.9_{-0.8}^{+1.1}$ & $240_{-36}^{+77}$ & $12.4_{-1.7}^{+2.4}$ \\
\hline$h e p$ & $2.10 \times 10^{3}$ & 390 & 0.0 & $714_{-114}^{+228}$ & 0.0 \\
\hline${ }^{13} \mathrm{~N}$ & $\left(6.05_{-0.77}^{+1.15}\right) \times 10^{8}$ & 0.017 & 0.1 & $0.604_{-0.018}^{+0.036}$ & $3.7_{-0.5}^{+0.7}$ \\
\hline${ }^{15} \mathrm{O}$ & $\left(5.32_{-0.80}^{+1.17}\right) \times 10^{8}$ & $0.068 \pm 0.001$ & $0.4 \pm 0.1$ & $1.137_{-0.057}^{+0.136}$ & $6.0{ }_{-0.9}^{+1.3}$ \\
\hline${ }^{17} \mathrm{~F}$ & $\left(6.33_{-0.70}^{+0.76}\right) \times 10^{6}$ & 0.069 & 0.0 & $1.139_{-0.057}^{+0.137}$ & 0.1 \\
\hline Total & & $7.7_{-1.0}^{+1.2}$ & & $129_{-6}^{+8}$ \\
\hline \hline
\end{tabular}

Table 5.2. Standard Solar Model [304] neutrino fluxes, average neutrino cross sections [60,317, 318] and SSM predictions for the neutrino capture rates [304] in the chlorine $(\mathrm{Cl})$ Homestake experiment and in the gallium (Ga) GALLEX and SAGE experiments.

The neutrino flux coming from each source as a function of the neutrino energy $E$ can be written as

$$
\phi_{r}(E)=\Phi_{r} X_{r}(E) \quad\left(r=p p, p e p,{ }^{7} \mathrm{Be},{ }^{8} \mathrm{~B}, h e p,{ }^{13} \mathrm{~N},{ }^{15} \mathrm{O},{ }^{17} \mathrm{~F}\right),
$$

where $\Phi_{r}$ is the total flux and $X_{r}(E)$ is the energy spectrum $\left(\int \mathrm{d} E X_{r}(E)=1\right)$. The energy spectrum $X_{r}(E)$ for each source $r$ is known with negligible uncertainties [317, 320] because it is determined by the weak interactions and it is practically independent from solar physics. On the other hand, the total flux $\Phi_{r}$ of each source $r$ must be calculated with a solar model and the resulting uncertainties represent one of the main problem for the interpretation of the experimental results. However, there are some relations that allow to extract model-independent information on the neutrino fluxes from the experimental data. The main one is the luminosity constraint, which is based on the assumption that the sun is in a stable state (the energy is produced in the central region of the sun and for its electromagnetic part it takes more than $10^{4}$ years to reach the surface, whereas neutrinos escape the sun in about two seconds). Let us consider a solar neutrino with energy $E$. The luminous energy released together with this neutrino is $Q / 2-E$. Multiplying this quantity with the total flux of neutrinos $\sum_{r} \phi_{r}(E)$ and 
integrating over the neutrino energy one obtains the luminosity constraint ${ }^{20}$

$$
\sum_{r}\left(\frac{Q}{2}-\langle E\rangle_{r}\right) \Phi_{r}=\frac{\mathcal{L}_{\odot}}{4 \pi R^{2}},
$$

where $\langle E\rangle_{r}=\int E X_{r}(E) \mathrm{d} E$ is the average energy of the neutrinos from the source $r$ (see Table 5.1], $\mathcal{L}_{\odot}=2.40 \times 10^{39} \mathrm{MeV} \mathrm{s}^{-1}$ [35] is the luminosity of the sun, $R=1.496 \times 10^{13} \mathrm{~cm}$ [35] is the sun - earth distance. The luminosity constraint can be rewritten in the compact form

$$
\sum_{r} Q_{r} \Phi_{r}=K_{\odot}
$$

where $Q_{r} \equiv Q / 2-\langle E\rangle_{r}$ and $K_{\odot} \equiv \mathcal{L}_{\odot} / 4 \pi R^{2}=8.54 \times 10^{11} \mathrm{MeV} \mathrm{cm}^{-2} \mathrm{~s}^{-1}$ is the solar constant. Let us emphasize that the luminosity relation is valid under the assumption that solar $\nu_{e}$ 's on their way to the earth do not transform into other states. Neglecting the small fraction of energy carried away by neutrinos, the luminosity constraint gives the approximate value of the total solar neutrino flux $\Phi=\sum_{r} \Phi_{r}$ :

$$
\Phi \simeq \frac{2 K_{\odot}}{Q}=6.4 \times 10^{10} \mathrm{~cm}^{-2} \mathrm{~s}^{-1} .
$$

Since the ${ }^{3} \mathrm{He}$ nuclei necessary for the formation of ${ }^{7} \mathrm{Be}$ and ${ }^{8} \mathrm{~B}$ are created by the $p p$ or pep reactions, there is another model-independent constraint for the solar neutrino fluxes of the $p p$ cycle (see [321]):

$$
\phi_{{ }^{\mathrm{Be}}}+\phi_{8_{\mathrm{B}}} \leq \phi_{p p}+\phi_{p e p} .
$$

Let us now consider the experimental data. The results of five solar neutrino experiments are available at present and are listed in Table 5.3.

Homestake 36 38], GALLEX [42, 43] and SAGE [44, 45] are radiochemical experiments. In the pioneering chlorine Homestake experiment of R. Davis et. al., which started in 1967, the detector is a tank with a volume of $6 \times 10^{5}$ liters filled with $\mathrm{C}_{2} \mathrm{Cl}_{4}$. Radioactive atoms of ${ }^{37} \mathrm{Ar}$ are produced by solar electron neutrinos through the reaction 324, 325]

$$
\nu_{e}+{ }^{37} \mathrm{Cl} \rightarrow e^{-}+{ }^{37} \mathrm{Ar},
$$

which has an energy threshold $E_{\mathrm{th}}=0.81 \mathrm{MeV}$. The radioactive ${ }^{37} \mathrm{Ar}$ atoms that are created during the time of exposition of each run (about two months) are extracted from the detector by purging it with ${ }^{4} \mathrm{He}$ and counted in small proportional counters which detect the Auger electron produced in the electron-capture of the ${ }^{37} \mathrm{Ar}$ nuclei. About 0.5 atoms of ${ }^{37} \mathrm{Ar}$ are produced every day by solar neutrinos and about 16 atoms are extracted in each run (this number is smaller than 30 because of the ${ }^{37} \mathrm{Ar}$ lifetime of about 35 days and because of the extraction efficiency of about 90\%). Since the energy threshold is

\footnotetext{
${ }^{20}$ The corrections to this relation due to the generation of gravitational energy 174 and due to the fact that the abundance of ${ }^{3} \mathrm{He}$ nuclei is out of equilibrium in the outer regions of the solar core 321. are estimated to be less than $1 \%$ and thus negligible at the present level of accuracy.
} 


\begin{tabular}{cccc} 
Experiment & Result & Theory & $\frac{\text { Result }}{\text { Theory }}$ \\
\hline \hline Homestake [38] & $\begin{array}{c}2.56 \pm 0.16 \pm 0.16 \\
(2.56 \pm 0.23)\end{array}$ & $7.7_{-1.0}^{+1.2}$ & $0.33_{-0.05}^{+0.06}$ \\
\hline GALLEX [322] & $\begin{array}{c}77.5 \pm 6.2_{-4.7}^{+4.3} \\
(78 \pm 8)\end{array}$ & $129_{-6}^{+8}$ & $0.60 \pm 0.07$ \\
\hline SAGE [323] & $\begin{array}{c}66.6_{-7.1-4.0}^{+6.8} \\
(67 \pm 8)\end{array}$ & $129_{-6}^{+8}$ & $0.52 \pm 0.07$ \\
\hline Kamiokande [41] & $\begin{array}{c}2.80 \pm 0.19 \pm 0.33 \\
(2.80 \pm 0.38)\end{array}$ & $5.15_{-0.7}^{+1.0}$ & $0.54 \pm 0.07$ \\
\hline Super-Kamiokande [48] & $\begin{array}{c}2.44 \pm 0.05_{-0.07}^{+0.09} \\
\left(2.44_{-0.09}^{+0.10}\right)\end{array}$ & $5.15_{-0.7}^{+1.0}$ & $0.47_{-0.09}^{+0.07}$ \\
\hline \hline
\end{tabular}

Table 5.3. The results of solar neutrino experiments confronted with the corresponding theoretical predictions [304]. The results of the Homestake, GALLEX and SAGE experiments are expressed in terms of event rates in SNU units ( $1 \mathrm{SNU} \equiv 10^{-36}$ eventsatom $^{-1} \mathrm{~s}^{-1}$ ), whereas the results of the Kamiokande and Super-Kamiokande experiments are expressed in terms of the ${ }^{8} \mathrm{~B}$ neutrino flux in units of $10^{6} \mathrm{~cm}^{-2} \mathrm{~s}^{-1}$. The first experimental error is statistical and the second is systematic. The experimental values in parenthesis have the statistical and systematic errors added in quadrature.

above the end-point of the $p p$ neutrino spectrum and the cross section of the detecting process (5.23) grows with the neutrino energy, the main contribution to the counting rate in the Homestake experiment comes from ${ }^{8} \mathrm{~B}$ and ${ }^{7} \mathrm{Be}$ neutrinos. According to the BP98 SSM [304] the event rate in the Homestake experiment should be $27.7_{-1.0}^{+1.2} \mathrm{SNU}$, of which $5.9 \mathrm{SNU}$ come from ${ }^{8} \mathrm{~B}$ neutrinos, $1.15 \mathrm{SNU}$ are produced by ${ }^{7} \mathrm{Be}$ neutrinos and $0.7 \mathrm{SNU}$ are due to pep and CNO neutrinos (see Table 5.2). The Homestake event rate presented in Table 5.3 is the event rate averaged over 108 runs [38].

In the radiochemical gallium experiments GALLEX and SAGE electron neutrinos from the sun are detected through the observation of radioactive ${ }^{71} \mathrm{Ge}$ that is produced in the process

$$
\nu_{e}+{ }^{71} \mathrm{Ga} \rightarrow e^{-}+{ }^{71} \mathrm{Ge} .
$$

The GALLEX detector is a tank containing 30.3 tons of ${ }^{71} \mathrm{Ga}$ (100 tons of a water solution of gallium chloride), whereas the SAGE experiment uses about 57 tons of ${ }^{71} \mathrm{Ga}$ in metallic form. The event rate measured in the GALLEX experiment is $0.699 \pm 0.069$ events per day. (Since the ${ }^{71} \mathrm{Ge}$ lifetime is about 16.5 days, only around 7 atoms of ${ }^{71} \mathrm{Ge}$ are extracted in each 3-weeks run.)

Since the threshold of the process (5.24) is $E_{\mathrm{th}}^{71} \mathrm{Ga}=0.233 \mathrm{MeV}$, neutrinos from all sources are detected in gallium experiments. According to the SSM, the contributions to the total predicted event rate from $p p,{ }^{7} \mathrm{Be}$ and ${ }^{8} \mathrm{~B}$ neutrinos are [304 $54 \%, 27 \%$ and $10 \%$, respectively.

As can be seen from Table 5.3, both gallium detectors measure an event rate that is

\footnotetext{
${ }^{21}$ One Solar Neutrino Unit (SNU) is defined as $10^{-36}$ events atom ${ }^{-1} \mathrm{~s}^{-1}$.
} 
about one half of the SSM prediction. The weighted average of the GALLEX and SAGE results yields the event rate

$$
S_{\mathrm{Ga}}^{\exp }=72.5 \pm 6 \mathrm{SNU} \text {. }
$$

Taking into account the experimental and theoretical errors, this rate differs from the SSM prediction by about seven standard deviations!

Both the GALLEX and SAGE detectors have been calibrated using an intense ${ }^{51} \mathrm{Cr}$ neutrino source. The ratio of observed and expected events is $0.93 \pm 0.08$ for GALLEX [326] and $0.95 \pm 0.12$ for SAGE [45]. These results demonstrate the absence of unexpected systematic errors at the $10 \%$ level in both experiments. In addition, the GALLEX Collaboration calibrated the detector by introducing a known number of radioactive ${ }^{71} \mathrm{As}$ atoms in the target solution [326, 327]. The atoms of ${ }^{71} \mathrm{Ge}$ resulting from ${ }^{71} \mathrm{As}$ decay have been extracted in the usual way, and the As tests prove, at the $1 \%$ level, the reliability of the technique [328] (the number of ${ }^{71} \mathrm{Ge}$ atoms produced in these tests is of the order of $10^{5}$, whereas the number produced in the ${ }^{51} \mathrm{Cr}$ tests is of the order of ten per day). As emphasized by the GALLEX Collaboration [326, 327], these results rule out the presence of unexpected radiochemical effects that could explain the deficit of solar $\nu_{e}$ 's measured by the gallium experiments.

It is necessary to emphasize that the gallium experiments are not only very important for the assessment of the solar neutrino problem but also for the theory of thermonuclear energy production in the sun: They have provided the first observation of low-energy solar neutrinos produced in the pp reaction that is the basic reaction of the pp cycle and, therefore, the first direct experimental confirmation of the theory of thermonuclear origin of solar energy production.

In the Kamiokande [39 41] and Super-Kamiokande 46 48 experiments waterCherenkov detectors are used for the detection of solar neutrinos through the observation of the Cherenkov light emitted by the recoil electrons in the elastic-scattering process

$$
\nu+e^{-} \rightarrow \nu+e^{-}
$$

Since the direction of the recoil electron is peaked in the direction of the incoming neutrino, water-Cherenkov detectors measure the direction of the neutrino flux and the results of Kamiokande and Super-Kamiokande have proven that there is a flux of high-energy neutrinos coming from the sun with an intensity about half of that predicted by the SSM.

The energy threshold of water-Cherenkov detectors is given by the threshold for the detection of the recoil electron in the reaction (5.26). It is higher than in other solar neutrino experiments because of the large background at low energies: $E_{\mathrm{th}}^{\mathrm{Kam}} \simeq 7 \mathrm{MeV}$ in the Kamiokande experiment and $E_{\text {th }}^{\mathrm{SK}} \simeq 6.5 \mathrm{MeV}$ in the Super-Kamiokande experiment. Therefore, only ${ }^{8} \mathrm{~B}$ neutrinos can be detected in water-Cherenkov experiments. If nothing happens to electron neutrinos during their trip from the core of the sun to the earth (i.e., no neutrino oscillations or other transitions), the results of the Kamiokande and SuperKamiokande experiments provide a measurement of the total flux $\Phi_{{ }_{8} \mathrm{~B}}$ of ${ }^{8} \mathrm{~B}$ neutrinos. Therefore, the results of the Kamiokande and Super-Kamiokande experiments are usually presented in terms of the measured flux of ${ }^{8} \mathrm{~B}$ neutrinos. In the Super-Kamiokande 
experiment it was found

$$
\Phi_{8 \mathrm{~B}}^{\mathrm{SK}}=\left(2.44_{-0.09}^{+0.10}\right) \times 10^{6} \mathrm{~cm}^{-2} \mathrm{~s}^{-1},
$$

which is about one half of the flux predicted by the SSM (see Table 5.3). ${ }^{2}$

From the experimental results and the most updated theoretical predictions 304 listed in Table 5.3 one can see that the observed event rates in all solar neutrino experiments are significantly smaller than the predicted rates (see also [329] where the comparison with other theoretical predictions is discussed). This discrepancy constitutes the solar neutrino problem. The SSM is robust and it has been recently tested in a convincing way by comparing its predicted value for the sound speed in the interior of the sun with precise helioseismological measurements (see 304, 315, 330 333]). However, it is clear that a model-independent proof of the existence of the solar neutrino problem would be more convincing. Such a model-independent approach has been discussed in several papers [308, 312, 321, 329, 334 343] using the model-independent luminosity constraint (5.20) and the fact that the energy spectra $X_{r}(E)$ Eq.(5.18) of the various neutrino sources are practically independent from solar physics [317, 320].

From the luminosity constraint it is possible to obtain a model-independent lower bound on the gallium event rate. Indeed, since $p p$ neutrinos have the smallest average energy and the $\nu_{e}{ }^{-71} \mathrm{Ga}$ cross section $\sigma_{\mathrm{Ga}}(E)$ increases with the neutrino energy $E$, we have

$$
S_{\mathrm{Ga}}=\int \sigma_{\mathrm{Ga}}(E) \sum_{r} \phi_{r}(E) \mathrm{d} E=\sum_{r}\left\langle\sigma_{\mathrm{Ga}}\right\rangle_{r} \Phi_{r} \geq\left\langle\sigma_{\mathrm{Ga}}\right\rangle_{p p} \Phi,
$$

where $\left\langle\sigma_{\mathrm{Ga}}\right\rangle_{r}=\int \sigma_{\mathrm{Ga}}(E) X_{r}(E) \mathrm{d} E$ is the average cross section of neutrinos from the source $r$ (see Table 5.2). Since the luminosity constraint (5.20) implies that

$$
Q_{p p} \Phi \geq K_{\odot}
$$

we obtain

$$
S_{\mathrm{Ga}} \geq \frac{\left\langle\sigma_{\mathrm{Ga}}\right\rangle_{p p} K_{\odot}}{Q_{p p}}=76 \pm 2 \mathrm{SNU}
$$

where we used the value of $\left\langle\sigma_{\mathrm{Ga}}\right\rangle_{p p}=11.7 \times 10^{-46} \mathrm{~cm}^{2}$ given in Table 5.2 and $Q_{p p}=$ 13.1 MeV.

The lower bound (5.30) is just compatible with the combined result (5.25) of the gallium experiments. This means that the results of the GALLEX and SAGE experiments can be explained if practically only $p p$ neutrinos are emitted by the sun. This possibility is incompatible with any solar model constrained by the helioseismological data [304, 315].

More stringent model-independent conclusions on the existence of the solar neutrino problem can be obtained by comparing the results of different solar neutrino experiments. If the survival probability of solar $\nu_{e}$ 's is equal to one, the result of the Super-Kamiokande experiment gives the value (5.27) for the flux of ${ }^{8} \mathrm{~B}$ neutrinos, whose contributions to the

\footnotetext{
${ }^{22}$ For recent Super-Kamiokande results on solar neutrinos see Note added.
} 
event rates of the chlorine and gallium experiments are

$$
\begin{aligned}
& S_{\mathrm{Cl}}^{8 \mathrm{~B}, \mathrm{SK}}=\left\langle\sigma_{\mathrm{Cl}}\right\rangle_{{ }_{8} \mathrm{~B}} \Phi_{8_{\mathrm{B}}}^{\mathrm{SK}}=2.78 \pm 0.27 \mathrm{SNU}, \\
& S_{\mathrm{Ga}}^{\mathrm{B}_{\mathrm{B}, \mathrm{SK}}}=\left\langle\sigma_{\mathrm{Ga}}\right\rangle_{{ }_{8} \mathrm{~B}} \Phi_{{ }_{8} \mathrm{~B}}^{\mathrm{SK}}=5.9_{-0.9}^{+1.9} \mathrm{SNU} .
\end{aligned}
$$

Subtracting the contribution (5.31) of ${ }^{8} \mathrm{~B} \nu_{e}$ 's from the event rate measured in the Homestake experiment (see Table 5.3), one obtains

$$
S_{\mathrm{Cl}}^{\exp }-S_{\mathrm{Cl}}^{8 \mathrm{~B}, \mathrm{SK}}=-0.22 \pm 0.35 .
$$

Since the quantity $S_{\mathrm{Cl}}-S_{\mathrm{Cl}}^{8 \mathrm{~B}}$ represents the contribution to the chlorine event rate due to pep, ${ }^{7} \mathrm{Be}$, hep and $\mathrm{CNO}$ neutrinos, it must be positive (or zero) but the result in Eq.(5.33) is only marginally compatible with a positive value (the probability is less than $26 \%$ ). Furthermore, the result Eq.(5.33) shows that the fluxes of intermediate energy neutrinos (pep, ${ }^{7} \mathrm{Be}$ and $\mathrm{CNO}$ neutrinos) are strongly suppressed with respect to the SSM prediction (see Table 5.2). This is incompatible with any solar model constrained by the helioseismological data [304, 315] and with the fact that, since both the reactions that produce ${ }^{7} \mathrm{Be}$ and ${ }^{8} \mathrm{~B}$ neutrinos originate from ${ }^{7} \mathrm{Be}$ nuclei (see Fig. 5.3), it is very difficult to have a suppression of the ${ }^{7} \mathrm{Be}$ neutrino flux (with respect to the SSM value) that is stronger than the suppression of the ${ }^{8} \mathrm{~B}$ neutrino flux. ${ }^{3}$

Let us consider now the gallium experiments. Subtracting the contribution of ${ }^{8} \mathrm{~B}$ neutrinos from the luminosity constraint (5.20), we have

$$
K_{\odot}-Q_{8_{\mathrm{B}}} \Phi_{8_{\mathrm{B}}}=\sum_{r \neq^{8} \mathrm{~B}} Q_{r} \Phi_{r} \leq Q_{p p} \sum_{r \neq^{8} \mathrm{~B}} \Phi_{r} .
$$

Hence, following the same reasoning as in Eq.(5.28) we obtain

$$
S_{\mathrm{Ga}}^{\min }=S_{\mathrm{Ga}}^{8_{\mathrm{B}, \mathrm{SK}}}+\left\langle\sigma_{\mathrm{Ga}}\right\rangle_{p p} \frac{K_{\odot}-Q_{8_{\mathrm{B}}} \Phi_{8_{\mathrm{B}}}^{\mathrm{SK}}}{Q_{p p}}=82_{-2}^{+3} \mathrm{SNU},
$$

and the contribution of pep, ${ }^{7} \mathrm{Be}$, hep and $\mathrm{CNO}$ neutrinos to the gallium event rate cannot be bigger than

$$
S_{\mathrm{Ga}}^{\exp }-S_{\mathrm{Ga}}^{\min }=-9.5 \pm 6 .
$$

This result is compatible with a positive value with a probability smaller than $6 \%$. Again, the result (5.36) implies that the fluxes of intermediate energy neutrinos (pep, ${ }^{7} \mathrm{Be}$ and CNO neutrinos) are strongly suppressed with respect to the SSM prediction, in contradiction with any solar model constrained by the helioseismological data and with the moderate suppression of the ${ }^{8} \mathrm{~B}$ neutrino flux.

Further model-independent methods for proving the existence of the solar neutrino problem on the basis of the data of solar neutrino experiments are nicely discussed in

\footnotetext{
${ }^{23}$ Non-standard solar models with low central temperature yield a suppression of the ${ }^{8} \mathrm{~B}$ neutrino flux that is stronger than that of the ${ }^{7} \mathrm{Be}$ neutrino flux (see [312] and references therein).
} 


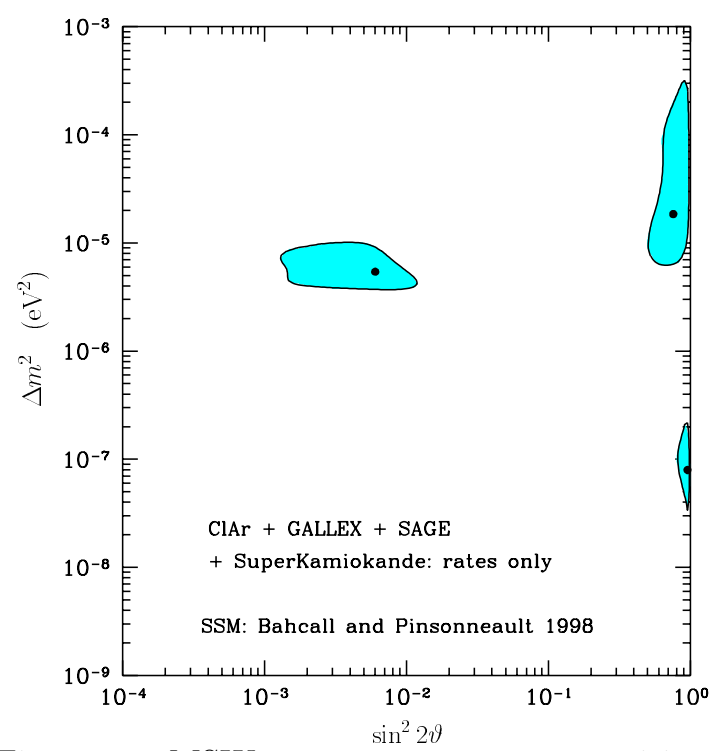

Figure 5.5. MSW $\nu_{e} \rightarrow \nu_{\mu}$ or $\nu_{e} \rightarrow \nu_{\tau}$ transitions: result of the fit of the experimental event rates listed in Table 5.3. The shadowed regions are allowed at 99\% CL 329]. The dots indicate the best-fit points in each allowed region.

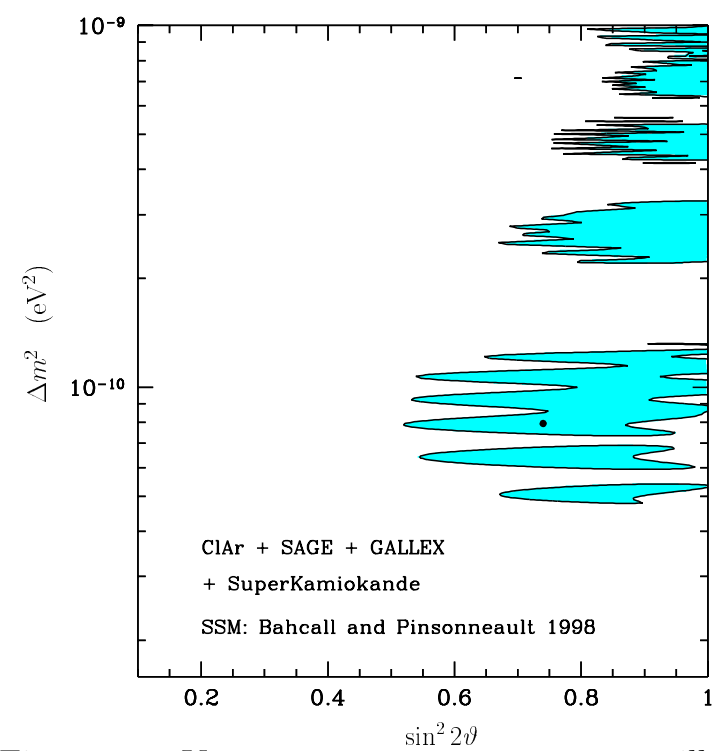

Figure 5.6. Vacuum $\nu_{e} \rightarrow \sin ^{2} 2 \vartheta$ or $\nu_{e} \rightarrow \nu_{\tau}$ oscillations: result of the fit of the experimental event rates listed in Table 5.3. The shadowed regions are allowed at $99 \% \mathrm{CL} 329$. The dot indicates the best-fit point.

Ref. [312]. In the following we will assume that there is a solar neutrino problem that is caused by neutrino oscillations.

The solar neutrino data have been analysed in many papers under the assumption of two-neutrino mixing (see [329, 342, 344] and references therein) and in a few papers under the assumption of three-neutrino mixing (see 345 348] and references therein). Here we will briefly review the results of the most updated two-generation analysis [329] of all the solar neutrino data, including those obtained in the first 504 days of operation of the Super-Kamiokande experiment (see Table 5.3).

The deficit of solar $\nu_{e}$ 's can be explained in terms of two-generation neutrino mixing either through vacuum oscillations or through MSW resonant transitions in matter [163, 164. Furthermore, the two mixed neutrinos can be the electron neutrino and another active $\left(\nu_{\mu}\right.$ or $\left.\nu_{\tau}\right)$ neutrino or the electron neutrino and a sterile neutrino. There are two differences between transitions of solar $\nu_{e}$ 's into active and sterile neutrinos:

1. The probability of MSW transitions is different in the two cases, because sterile neutrinos do not have the weak-interaction potential due to elastic forward scattering that is present for active neutrinos.

2. The active neutrinos $\left(\nu_{\mu}\right.$ 's or $\nu_{\tau}$ 's) that are produced by the oscillation mechanism (either in vacuum or in matter) contribute to the event rates measured in the Kamiokande and Super-Kamiokande water-Cherenkov experiments (notice, however, that the cross section of $\nu_{\mu}-e^{-}$and $\nu_{\tau}-e^{-}$scattering is about six times smaller than the $\nu_{e}-e^{-}$ cross section).

The formulas for the survival probability of solar $\nu_{e}$ 's in the case of vacuum oscillations 
and MSW transitions are given in Eq. 3.21) (with $\alpha=e$ ) and in Eq.4.41) together with Eq.(4.47) 193, respectively. These formulas depend on the two mixing parameters $\Delta m^{2}$ and $\sin ^{2} 2 \vartheta$. The allowed regions in the $\sin ^{2} 2 \vartheta-\Delta m^{2}$ plane, obtained in Ref. [329] from the fit of the measured event rates listed in Table 5.3 by using the BP98 SSM [304] and the analytic formulas in Ref. [193] for the MSW case, are shown in Figs. 5.5 5.6.

Figure 5.5 329] shows the three allowed regions in the case of MSW $\nu_{e} \rightarrow \nu_{\mu}$ or $\nu_{e} \rightarrow \nu_{\tau}$ transitions. They are the small mixing angle (SMA-active) region at

$$
\Delta m^{2} \simeq 5 \times 10^{-6} \mathrm{eV}^{2}, \quad \sin ^{2} 2 \vartheta \simeq 6 \times 10^{-3} \quad \text { (SMA-active) },
$$

and the large mixing angle (LMA) region at

$$
\Delta m^{2} \simeq 2 \times 10^{-5} \mathrm{eV}^{2}, \quad \sin ^{2} 2 \vartheta \simeq 0.76 \quad \text { (LMA) }
$$

The best fit, with a confidence level of $19 \%$, is obtained in the SMA region, whereas the LMA region has a confidence level of $4 \%$. In Ref. [329] there is also the so-called low mass (LOW) region at $\Delta m^{2} \simeq 8 \times 10^{-8} \mathrm{eV}^{2}$ and $\sin ^{2} 2 \vartheta \simeq 0.96$, however, the LOW region is only marginally acceptable, with a confidence level of $0.7 \%$.

The allowed regions in the $\sin ^{2} 2 \vartheta-\Delta m^{2}$ plane in the case of $\nu_{e} \rightarrow \nu_{\mu}$ or $\nu_{e} \rightarrow \nu_{\tau}$ vacuum oscillations are shown in Fig. 5.5 [329]. These regions extend over large ranges of $\sin ^{2} 2 \vartheta$ and $\Delta m^{2}$ around the best fit values

$$
\Delta m^{2} \simeq 8 \times 10^{-11} \mathrm{eV}^{2}, \quad \sin ^{2} 2 \vartheta \simeq 0.75 \quad \text { (Vac. Osc.) }
$$

The confidence level of the fit is $3.8 \%$. There is no allowed region in the case of $\nu_{e} \rightarrow \nu_{s}$ vacuum oscillations (the fit has a confidence level of 0.05\%) [329, 349].

As shown in Fig. 5.7 [329], only the small mixing angle (SMA-sterile) region at

$$
\Delta m^{2} \simeq 4 \times 10^{-6} \mathrm{eV}^{2}, \quad \sin ^{2} 2 \vartheta \simeq 7 \times 10^{-3} \quad \text { (SMA-sterile) },
$$

is allowed in the case of MSW $\nu_{e} \rightarrow \nu_{s}$ transitions, with a confidence level of $19 \%$ (the large mixing angle and low mass solutions have a confidence level of $0.001 \%$ and $0.003 \%$, respectively) 329, 350.

The authors of Ref. [329] performed also global fits of the solar neutrino data including the energy spectrum and zenith-angle distribution of the recoil electrons measured in the Super-Kamiokande experiment [48]. As noted in Ref. [329], since these data are still preliminary, the results of this analysis are less robust than those obtained fitting only the global rates. However, it is interesting to note that in the case of MSW $\nu_{e} \rightarrow \nu_{\mu}$ or $\nu_{e} \rightarrow \nu_{\tau}$ transitions only the SMA-active region remains allowed, as shown in Fig. 5.8 [329], with a confidence level of $7 \%$.

During the night, solar neutrinos pass through the earth and the matter effect can cause a regeneration of $\nu_{e}$ 's (see Ref. [316] and references therein) and, therefore, a zenith angle dependence of the solar neutrino flux. The size of this effect depends on the values of $\Delta m^{2}$ and $\sin ^{2} 2 \vartheta$ and is sizable only for large values of the mixing angle. The 


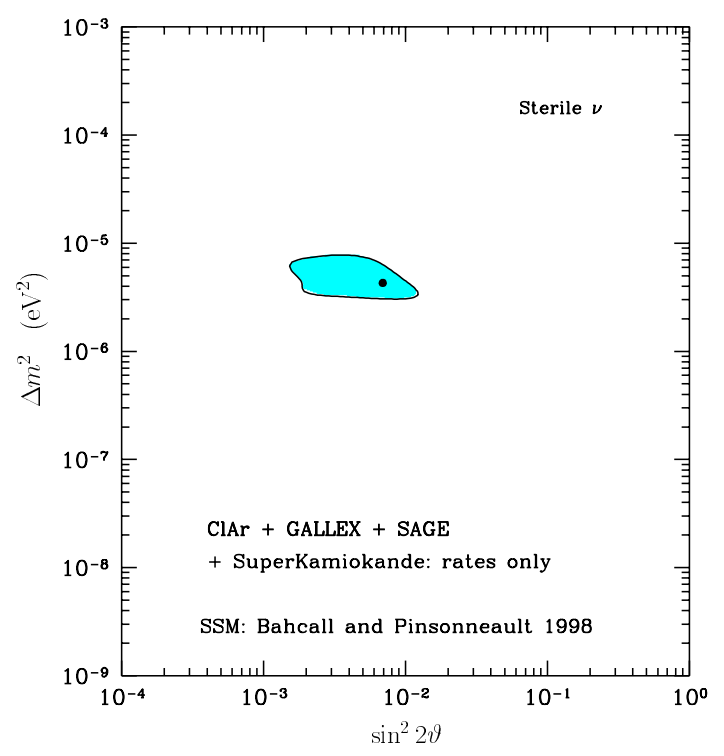

Figure 5.7. MSW $\nu_{e} \rightarrow \nu_{s}$ transitions: result of the fit of the experimental event rates listed in Table 5.3. The shadowed region is allowed at $99 \%$ CL [329]. The dot indicates the best-fit point.

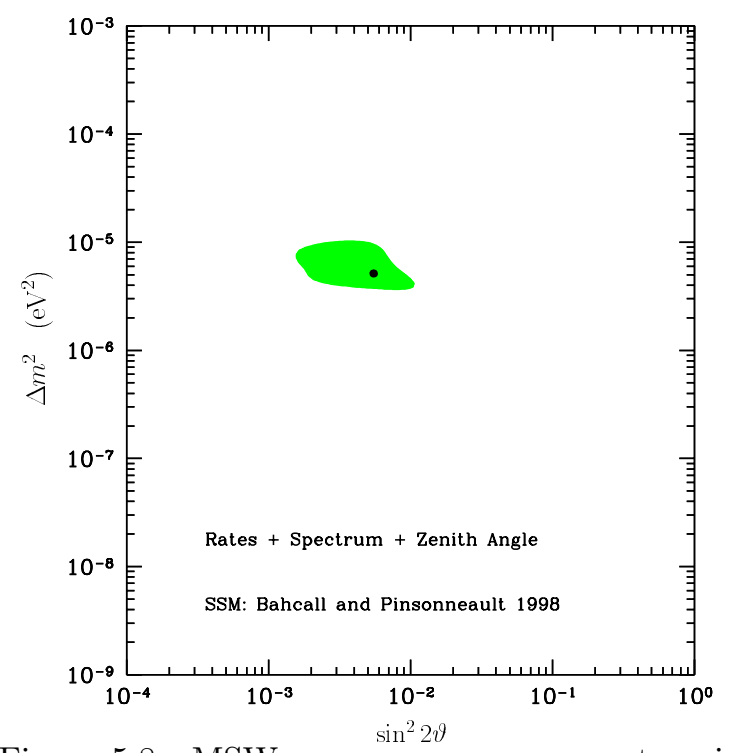

Figure 5.8. MSW $\nu_{e} \rightarrow \nu_{\mu}$ or $\nu_{e} \rightarrow \nu_{\tau}$ transitions: result of the global fit of the experimental event rates listed in Table 5.3 and of the energy spectrum and zenith-angle distribution measured in the Super-Kamiokande experiment [48]. The shadowed region is allowed at 99\% CL [329. The dot indicates the best-fit point.

preliminary value of the day-night asymmetry of solar neutrino events measured in the Super-Kamiokande experiment is 48]

$$
\frac{D-N}{D+N}=-0.023 \pm 0.020 \pm 0.014
$$

compatible with zero. Hence this experimental result tends to exclude solutions of the solar neutrino problem with a large mixing angle.

As was shown in Refs. [283, 351] the step-like profile of the matter density of the earth could lead to an enhancement of neutrino transitions not only for atmospheric neutrinos (see Section 5.1.3) but also for solar neutrinos.

In conclusion of this section, we would like to emphasize that the results of solar neutrino experiments provide a rather strong indication on favour of neutrino mixing and several experiments are under construction [273, 322, 352] or in project (see [353, 354] and references therein). In particular, the measurement of the electron neutrino spectrum at SNO may allow to obtain model-independent information on the neutrino oscillation probability and on the flux $\Phi_{8_{\mathrm{B}}}$ of ${ }^{8} \mathrm{~B}$ neutrinos [355 359], the measurement of the flux of ${ }^{7} \mathrm{Be}$ neutrinos on the earth in the Borexino experiment and the results of SuperKamiokande, SNO, Borexino and GNO will allow to distinguish the different possible solutions of the solar neutrino problem (see Refs. [350, 360 363] and references therein).

The Sudbury Neutrino Observatory (SNO) 273, 364] is located 6800 feet below ground in the Creighton mine, near Sudbury in Ontario (Canada). In the SNO experiment a Cherenkov detector with 1 kton of heavy water $\left(\mathrm{D}_{2} \mathrm{O}\right)$ contained in an acrylic vessel of 
$12 \mathrm{~m}$ diameter will be used. The Cherenkov light is detected with a geodesic array of $10^{4}$ photomultiplier tubes surrounding the heavy water vessel. The heavy water detector is immersed in normal water in order to reduce the background. Solar neutrinos will be observed in real-time through the charged-current (CC), neutral-current (NC) and elastic scattering (ES) reactions

$$
\begin{array}{ccc}
\nu_{e}+d \rightarrow e^{-}+p+p & & (\mathrm{CC}), \\
\nu_{\ell}+d \rightarrow \nu_{\ell}+p+n & , & \ell=e, \mu, \tau,
\end{array}
$$

Since the energy threshold for the observation of the recoil electrons in the CC and ES processes is about $5 \mathrm{MeV}$ and the neutrino energy threshold for the $\mathrm{NC}$ reaction is 2.2 $\mathrm{MeV}$, only ${ }^{8} \mathrm{~B}$ neutrinos can be observed. The event rates predicted by the SSM are around 23 per day for the CC reaction, 7 per day for the NC reaction and 3 per day for the ES reaction. From the hit pattern of the photomultipliers, the direction and energy of the neutrino in the $\mathrm{CC}$ reaction can be reconstructed, allowing a direct measurement of the spectrum of the solar electron neutrino flux on the earth. The observation of a distortion of this spectrum with respect to the one calculated without neutrino oscillations will represent a model-independent proof of the occurrence of neutrino oscillations. The NC reaction will allow to detect all active neutrinos with the same cross section (because of the $e-\mu-\tau$ universality of weak interactions), whereas the cross sections of $\nu_{\mu}$ and $\nu_{\tau}$ scattering in the ES reaction is about six times smaller than that of $\nu_{e}$. The measurement of the neutral current reaction will allow to determine the total flux of active neutrinos from the sun [355, 359], which coincides with the produced flux if there are only activeactive neutrino oscillations, which can be revealed trough a measurement of a deficit in the $\mathrm{NC} / \mathrm{CC}$ event rate. On the other hand, if the $\mathrm{NC} / \mathrm{CC}$ event rate will not show any deficit and a distortion of the electron neutrino spectrum will be observed, it will mean that there are transitions of solar $\nu_{e}$ 's into sterile states 356 (of course, complicated scenarios with transitions solar $\nu_{e}$ 's into active and sterile states are not excluded and must be taken into account). The SNO experiment is expected to release the first results in the year 1999.

The Borexino experiment 352, 365] is designed to detect low-energy solar neutrinos in real-time through the observation of the elastic scattering process (5.44) with an energy threshold for the recoil electron of $250 \mathrm{keV}$. It will use 300 tons of liquid scintillator in an unsegmented detector with 2000 photomultiplier tubes. The event rate predicted by the SSM for a fiducial volume of about 100 tons is about 50 events per day, mostly generated by the $0.86 \mathrm{MeV}$ monoenergetic line of ${ }^{7} \mathrm{Be}$ solar neutrinos. Since this line gives a characteristic spectral signature in the ES process, the flux on the earth of ${ }^{7} \mathrm{Be}$ solar neutrinos will be determined and it will be possible to check if it is suppressed with respect to the one predicted by the SSM as suggested by the results of current experiments. The neutrino oscillation solutions predict a measurable flux: $\sim 10$ events per day for the SMA MSW solution, $\sim 30$ events per day for the LMA MSW solution and $\sim 35$ events per day for the vacuum oscillation solution (see [360]). The Borexino experiment is under construction in the Laboratori Nazionali del Gran Sasso in Italy and 
is scheduled to start data taking around the year 2001.

\subsection{The LSND experiment}

The Liquid Scintillator Neutrino Detector (LSND) experiment [53, 54, 366 371] at the Los Alamos Meson Physics Facility (LAMPF) is a SBL neutrino oscillation experiment with a baseline of about 30 meters. It utilizes the LAMPF proton beam with a kinetic energy of $800 \mathrm{MeV}$ which produces pions by hitting a $30 \mathrm{~cm}$ long water target located about $1 \mathrm{~m}$ upstream from a copper beam stop. Most of the $\pi^{+}$'s are stopped in the target and decay into muons which come to rest and decay in the target as well. The decay at rest of the positively charged muons allows to investigate $\bar{\nu}_{\mu} \rightarrow \bar{\nu}_{e}$ oscillations [53, 366, 367, 370, 371 . A small fraction of the positively charged pions $(\sim 3.4 \%)$ decays in flight in the $1 \mathrm{~m}$ long space between the target and the beam stop and is used for the study of $\nu_{\mu} \rightarrow \nu_{e}$ oscillations [54, 369 371] (only $\sim 0.001 \%$ of the $\mu^{+}$'s decay in flight and produce a small contamination of $\nu_{e}$ 's in the $\nu_{\mu}$ beam). The detector is an approximately cylindrical tank $8.3 \mathrm{~m}$ long by $5.7 \mathrm{~m}$ in diameter, filled with a scintillator medium consisting mainly of mineral oil $\left(\mathrm{CH}_{2}\right)$. In the following we review the results obtained by the LSND Collaboration from the analyses of the data from $\mu^{+}$decay at rest and $\pi^{+}$decay in flight.

$\mu^{+}$decay at rest $53,366,367,370,371$. In this case one has the decay chain

$$
\begin{aligned}
\pi^{+} \rightarrow & \mu^{+}+\nu_{\mu} \\
& \downarrow \\
& e^{+}+\nu_{e}+\bar{\nu}_{\mu} .
\end{aligned}
$$

Note that in these decays no $\bar{\nu}_{e}$ appears, which makes the study of $\bar{\nu}_{\mu} \rightarrow \bar{\nu}_{e}$ transitions possible. In $\mu^{+}$decay at rest the upper bound on the $\bar{\nu}_{\mu}$ energy is $m_{\mu} / 2=52.8 \mathrm{MeV}$ ( $m_{\mu}$ is the muon mass) and the energy spectrum of the $\bar{\nu}_{\mu}$ flux is very well known, being determined by the kinematics of muon decay. The electron antineutrinos are detected by

$$
\bar{\nu}_{e}+p \rightarrow e^{+}+n
$$

The $\bar{\nu}_{e}$ signature is a coincidence between $e^{+}$and a delayed $\gamma$ from the capture $n+p \rightarrow$ $d+\gamma(2.2 \mathrm{MeV})$.

There is a background of $\bar{\nu}_{e}$ from the decay chain symmetrical to Eq. (5.45) starting with $\pi^{-}$. This $\nu_{e}$ flux is suppressed relative to the $\bar{\nu}_{\mu}$ flux from the $\pi^{+}$chain by a factor of $7.8 \times 10^{-4}$ for three reasons: $\pi^{-}$production in the beam stop is a factor of 8 smaller than $\pi^{+}$production, only $5 \%$ of the $\pi^{-}$decay in flight (all stopped $\pi^{-}$are absorbed) and of the resulting $\mu^{-}$only $12 \%$ decay before they are captured by nuclei. In the latter case they give rise to $\nu_{\mu}$ but not to $\bar{\nu}_{e}$. The LSND apparatus does not distinguish between $e^{+}$ and $e^{-}$. Therefore, the reaction $\nu_{e}+{ }^{12} C \rightarrow e^{-}+n+{ }^{11} N$ gives a signal like a $\bar{\nu}_{e}$ but can only occur for $E_{e}<20 \mathrm{MeV}$.

LSND has seen an excess of $e^{+}$events with energies between 20 and $60 \mathrm{MeV}$ of $51.0_{-19.5}^{+20.2} \pm 8.0$ corresponding to a $\bar{\nu}_{\mu} \rightarrow \bar{\nu}_{e}$ transition probability of $(3.1 \pm 1.2 \pm 0.5) \times 10^{-3}$ [53]. In Refs. [370, 371] preliminary results can be found for the data taken from 1993 to 


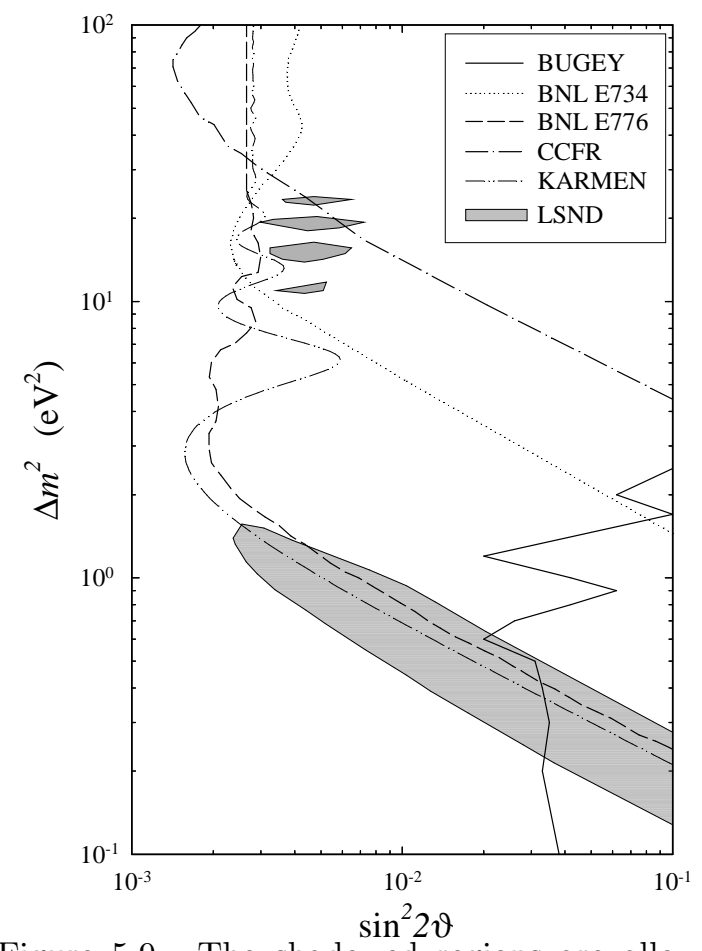

Figure 5.9. The shadowed regions are allowed at $90 \%$ CL by the $\mu^{+}$decay at rest analysis of the LSND data [370, 371]. Also shown are the exclusion curves of the Bugey 162], BNL E734 372], BNL E776 373, CCFR 374 and KARMEN (Bayesian analysis) 375 experiments.

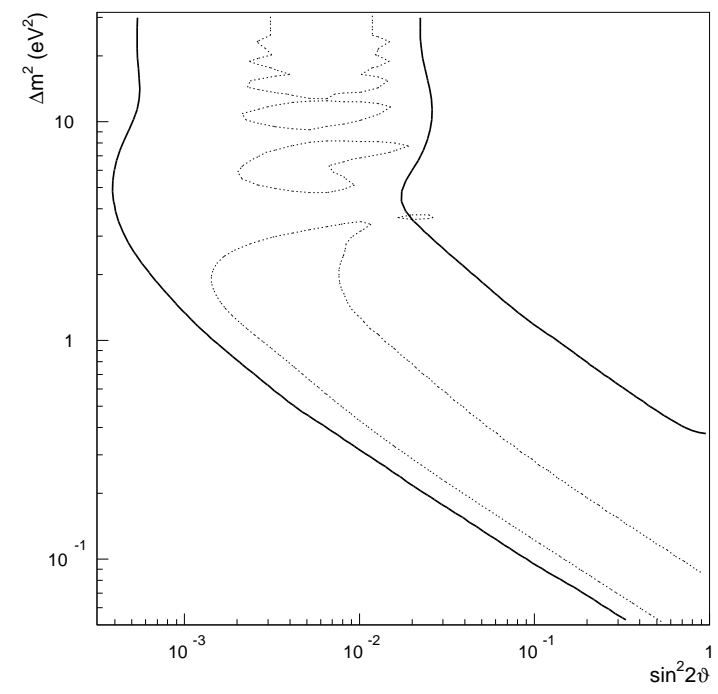

Figure 5.10. The region between the two solid lines is allowed at 95\% CL by the $\pi^{+}$decay in flight analysis of the LSND data [54, 369]. The regions enclosed by dashed lines are the 99\% CL LSND favoured regions obtained with the 1996 muon decay at rest analysis [53, 367].

1997. The value for the transition probability agrees with the previous one, except for a reduced statistical error of $0.9 \times 10^{-3}$ instead of $1.2 \times 10^{-3}$. The plot of the favoured region in the $\sin ^{2} 2 \vartheta-\Delta m^{2}$ plane obtained by the LSND collaboration is shown in Fig. 5.9. In the positron energy range between 36 and $60 \mathrm{MeV}$ the background is reduced because in this energy range there are no electrons from $\nu_{e}+{ }^{12} C \rightarrow e^{-}+{ }^{12} N$ induced by electron neutrinos from $\mu^{+}$decay at rest [53]. LSND has observed 22 events with such positron energies compared to a background of only $4.6 \pm 0.6$ events [53].

$\pi^{+}$decay in flight [54,369 371]. The $\pi^{+} \rightarrow \mu^{+}+\nu_{\mu}$ decay in flight generates a $\nu_{\mu}$ beam with which $\nu_{\mu} \rightarrow \nu_{e}$ oscillations are studied. The electron neutrinos are detected by electrons from the inclusive reaction

$$
\nu_{e}+{ }^{12} C \rightarrow e^{-}+X
$$

where the electrons have an energy $E_{e}$ between 60 and $200 \mathrm{MeV}$. In this case the signal is a single electron. The electron neutrinos from $\mu^{+} \rightarrow e^{+}+\nu_{e}+\bar{\nu}_{\mu}$ decay in flight are suppressed by the longer muon lifetime and the kinematics of the three-body decay, whereas those from $\pi^{+} \rightarrow e^{+}+\nu_{e}$ by the small branching ratio of $1.23 \times 10^{-4}$. The lower bound of $60 \mathrm{MeV}$ on $E_{e}$ lies well above $m_{\mu} / 2=52.8 \mathrm{MeV}$, the endpoint of the electron 
spectrum from muon decay at rest. Such muons can be induced by beam-related $\stackrel{(-)}{\nu}$ events. Also $\nu_{e}$ from $\mu^{+}$decay at rest (5.45) generate electrons below this energy. LSND has seen 40 electron events compared to a background of $21.9 \pm 2.1$. This corresponds to a $\nu_{\mu} \rightarrow \nu_{e}$ transition probability of $(2.6 \pm 1.0 \pm 0.5) \times 10^{-3}$ [54], consistent with the one determined by muon decay at rest. The favoured region in the $\sin ^{2} 2 \vartheta-\Delta m^{2}$ plane obtained by the LSND collaboration is shown in Fig. 5.10 as the area between the two solid lines [54. The regions in Fig. 5.10 enclosed by dashed lines are the LSND favoured regions obtained with the 1996 muon decay at rest analysis [53, 367]. One can see that the two results are perfectly compatible.

Recently, the LSND collaboration has reported [376] new evidence in favour of $\nu_{\mu} \rightarrow \nu_{e}$ oscillations using the $\nu_{\mu}$ beam produced by $\pi^{+}$decay in flight and detecting the $\nu_{e}$ 's through the observation of the reaction

$$
\nu_{e}+{ }^{12} \mathrm{C} \rightarrow{ }^{12} \mathrm{~N}_{\text {g.s. }}+e^{-}
$$

where ${ }^{12} \mathrm{~N}_{\text {g.s. }}$. is the ground state of ${ }^{12} \mathrm{~N}$, which decays with a lifetime of $15 \mathrm{~ms}$ into ${ }^{12} \mathrm{C}+e^{+}+\nu_{e}$. A temporal $(\lesssim 15 \mathrm{~ms})$ and spatial $(\lesssim 30 \mathrm{~cm})$ correlation between the observations of the $e^{-}$and $e^{+}$allows an almost background-free measurement. The LSND collaboration reported 376] the observation of 5 events, with an expected background of 0.5 events. These numbers are in agreement with the results of the other two analyses of the LSND data summarized above.

The region of neutrino mixing parameters indicated by the results of the LSND experiments is presently explored also by the KARMEN experiment 375, 377 382. The KARMEN experiment is located at the ISIS spallation neutron facility of the Rutherford Laboratories (UK). At ISIS a pulsed proton beam is used: two $100 \mathrm{~ns}$ wide proton pulses separated by $330 \mathrm{~ns}$ are produced every $20 \mathrm{~ms}$. The proton pulses are directed on a target where they produce positive pions (the negative pions are absorbed in the source before they decay) which decay at rest according to the chain (5.45) producing an equal number of $\nu_{\mu}, \nu_{e}$ and $\bar{\nu}_{\mu}$. The KARMEN Collaboration searches for $\bar{\nu}_{e}$ at a distance of about 18 $\mathrm{m}$. The time structure of the neutrino beam is important for the identification of the neutrino induced reactions in the KARMEN detector and for the effective suppression of the cosmic ray background. The KARMEN experiment started in 1990 and ran until 1995 as KARMEN 1 378. In 1996 the experiment was upgraded to KARMEN 2, eliminating the main cosmic ray induced background component in the search for $\bar{\nu}_{\mu} \rightarrow \bar{\nu}_{e}$ oscillations [379]. So far, the KARMEN 2 experiment measured no events 375, 380 382], also no background events, of which $2.88 \pm 0.13$ events were expected. The resulting exclusion curve obtained with a Bayesian analysis 375] is shown in Fig. 5.9. One can see that the null result of the KARMEN 2 experiment excludes part of LSND-allowed region in favour of $\bar{\nu}_{\mu} \rightarrow \bar{\nu}_{e}$ oscillations, but at present the sensitivity of the KARMEN experiment is not enough to check all the LSND-allowed region. ${ }^{24}$

${ }^{24}$ The KARMEN 2 result has also been analysed using the Unified Approach [383, which is a frequentist method with proper coverage (see Ref. 384). The resulting exclusion curve seems to forbid almost all of the LSND-allowed region [380, 382], but this result depends on the non-observation of the expected background events. The analysis of the KARMEN 2 null result with an alternative frequentist method 
The KARMEN experiment is expected to reach a sensitivity that will allow to cover the LSND-allowed region in one or two years [379]. If the KARMEN experiment will find an oscillation signal compatible with the one observed in the LSND experiment, the LSND evidence in favour of $\stackrel{(-)}{\nu_{\mu}} \rightarrow \stackrel{(-)}{\nu}$ oscillations will be confirmed. On the other hand, if the KARMEN experiment will not find any neutrino oscillation signal, then the KARMEN exclusion curve will lie on the left of the LSND allowed region in the $\sin ^{2} 2 \vartheta-\Delta m^{2}$ plane, but not far away from it [379]. Hence, it is important to test the LSND result with a new experiment with much higher sensitivity. Four such experiments have been proposed and are under study: BooNE [387] at Fermilab, I-216 [388] at CERN, ORLaND [389] at Oak Ridge and NESS at the European Spallation Source [390]. Among these proposals BooNE is approved and will start in 2001.

We would like to finish this section by emphasizing the importance of the results obtained in the LSND experiment for neutrino physics. As we will discuss in the next section, the LSND evidence in favour of SBL $\stackrel{(-)}{\nu} \rightarrow \stackrel{(-)}{\nu}$ oscillations taken together with the indications in favour of solar and atmospheric neutrino oscillations require the existence of at least one sterile neutrino, which represents a manifestation of new physics beyond the Standard Model. Hence it is important that the LSND indication is checked as soon as possible by another experiment with high sensitivity.

\section{Analysis of neutrino oscillation data}

As we have discussed in the previous section, at present there are three indications in favour of non-zero neutrino masses and mixing. The analysis of the experimental data (based on the simplest assumption of two neutrino mixing) indicate that there must be at least three different $\Delta m^{2}$, each one relevant for the oscillations of neutrinos in one type of experiments: $\Delta m^{2} \sim 10^{-5} \mathrm{eV}^{2}$ (MSW) or $\Delta m^{2} \sim 10^{-10} \mathrm{eV}^{2}$ (Vac. Osc.) is relevant for oscillations of solar neutrinos, $\Delta m^{2} \sim 10^{-3} \mathrm{eV}^{2}$ is relevant for oscillations of atmospheric neutrinos, and $\Delta m^{2} \sim 1 \mathrm{eV}^{2}$ is relevant for SBL neutrino oscillations according to the LSND experiment. Three different $\Delta m^{2}$ require the mixing of at least four massive neutrinos. Here we consider two possible scenarios:

1. Only the indications in favour of neutrino mixing obtained in atmospheric and solar neutrino experiments are confirmed by future experiments. In this case the mixing of three massive neutrinos can accommodate all data.

2. All the existing indications in favour of neutrino mixing, including that obtained in the LSND experiment, are confirmed by future experiments. In this case, the minimal mixing scheme that allows to accommodate all data is one with mixing of four neutrinos.

with proper coverage 385 leads to an exclusion curve compatible with the Bayesian exclusion curve shown in Fig. 5.9 [385, 386.

${ }^{25}$ From time to time special schemes with three neutrinos have been proposed in order to accommodate all neutrino oscillation data 391 397. However, none of these schemes can fit all data in a satisfactory way 


\subsection{Mixing of three massive neutrinos}

Let us consider the mixing of three massive neutrinos, i.e., $n=3$ in Eqs.(3.3) and (3.9),

$$
\left|\nu_{\alpha}\right\rangle=\sum_{k=1}^{3} U_{\alpha k}^{*}\left|\nu_{k}\right\rangle, \quad\left|\bar{\nu}_{\alpha}\right\rangle=\sum_{k=1}^{3} U_{\alpha k}\left|\bar{\nu}_{k}\right\rangle \quad(\alpha=e, \mu, \tau),
$$

and assume the neutrino mass hierarchy

$$
m_{1} \ll m_{2} \ll m_{3}
$$

that is indicated by the see-saw mechanism (see Section 2.5) and by the models beyond the Standard Model (see Section 2.6). In this case, the only available possibility for the explanation of the solar and atmospheric neutrino anomalies through neutrino oscillations is that $\Delta m_{21}^{2} \equiv m_{2}^{2}-m_{1}^{2}$ is relevant for the transitions of solar neutrinos and $\Delta m_{31}^{2} \equiv$ $m_{3}^{2}-m_{1}^{2}$ is relevant for the transitions of atmospheric neutrinos:

$$
\begin{aligned}
& \Delta m_{21}^{2} \sim 10^{-5} \mathrm{eV}^{2}(\mathrm{MSW}) \quad \text { or } \quad \Delta m_{21}^{2} \sim 10^{-10} \mathrm{eV}^{2} \text { (Vac. Osc.) } \\
& \Delta m_{31}^{2} \sim 10^{-3} \mathrm{eV}^{2} .
\end{aligned}
$$

Let us consider the oscillations of atmospheric and LBL neutrinos and assume that

$$
\frac{\Delta m_{21}^{2} L}{E} \ll 1
$$

The distance $L$ travelled by atmospheric neutrinos ranges from $\sim 10-20 \mathrm{~km}$ for downward-going neutrinos to $\sim 1.3 \times 10^{4} \mathrm{~km}$ for upward-going neutrinos and the energy $E$ relevant for the oscillations of atmospheric neutrino experiments ranges from $\sim 100 \mathrm{MeV}$ to $\sim 100 \mathrm{GeV}$. Therefore, if the solar neutrino problem is due to MSW transitions, the condition (6.5) is not satisfied by atmospheric neutrinos with energies below $\sim 1 \mathrm{GeV}$. In this case, the following discussion applies only to high-energy atmospheric neutrinos. On the other hand, the inequality (6.5) is valid in any case for reactor LBL experiments with a source - detector distance $L \lesssim 1 \mathrm{~km}$ and for accelerator LBL experiments with a source - detector distance $L \lesssim 10^{3} \mathrm{~km}$ and neutrino energy $E \gtrsim 1 \mathrm{GeV}$.

If the inequality (6.5) is satisfied, from Eq.(3.12), we have for the probability of $\nu_{\alpha} \rightarrow \nu_{\beta}$ transitions

$$
P_{\nu_{\alpha} \rightarrow \nu_{\beta}}^{(\operatorname{atm}, \mathrm{LBL})}=\left|\delta_{\alpha \beta}+U_{\alpha 3}^{*} U_{\beta 3}\left[\exp \left(-i \frac{\Delta m_{31}^{2} L}{2 E}\right)-1\right]\right|^{2} .
$$

[398, 399]. Schemes with more than four neutrinos have been considered in the papers in Refs. 130, 400 (see the review Ref. 401).

${ }^{26}$ Actually, the phenomenology of neutrino oscillations discussed in this section depends only on the hierarchy $\Delta m_{21}^{2} \ll \Delta m_{32}^{2}$ of mass-squared differences. Hence, the discussion of neutrino oscillations presented in this section applies, for example, also to the case of three almost degenerate neutrinos with masses in the $\mathrm{eV}$ range that give a contribution to the dark matter in the universe. Furthermore, with a cyclic permutation of the indices $1,2,3$, the discussion of neutrino oscillations presented in this section can be applied to all cases in which $\Delta m_{32}^{2} \ll \Delta m_{21}^{2}$, as, for example, the case of an inverted mass hierarchy $m_{1} \ll m_{2} \simeq m_{3}$. 
Therefore, a mass hierarchy of three neutrinos implies that the transition probabilities in atmospheric and LBL experiments depend only on the largest mass squared difference $\Delta m_{31}^{2}$ and on the elements $U_{\alpha 3}$ that connect flavour neutrinos with the heaviest neutrino $\nu_{3}$. From Eq.(6.6) we obtain [192, 402, 405]

$$
\begin{aligned}
& P_{\nu_{\alpha} \rightarrow \nu_{\beta}}^{(\operatorname{atm}, \mathrm{LBL})}=\frac{1}{2} A_{\alpha ; \beta}\left(1-\cos \frac{\Delta m_{31}^{2} L}{2 E}\right) \quad(\alpha \neq \beta), \\
& P_{\nu_{\alpha} \rightarrow \nu_{\alpha}}^{(\operatorname{atm}, \mathrm{LBL})}=1-\frac{1}{2} B_{\alpha ; \alpha}\left(1-\cos \frac{\Delta m_{31}^{2} L}{2 E}\right),
\end{aligned}
$$

with the oscillation amplitudes

$$
\begin{aligned}
& A_{\alpha ; \beta}=4\left|U_{\alpha 3}\right|^{2}\left|U_{\beta 3}\right|^{2}, \\
& B_{\alpha ; \alpha}=4\left|U_{\alpha 3}\right|^{2}\left(1-\left|U_{\alpha 3}\right|^{2}\right) .
\end{aligned}
$$

The expressions (6.7) and (6.8) have the same dependence on the quantity $\Delta m_{31}^{2} L / 2 E$ as the standard expressions (3.20) and (3.21) that describe neutrino oscillations in the case of two flavours, with $\Delta m^{2}$ replaced by $\Delta m_{31}^{2}$. This is due to the fact that only one masssquared difference is relevant for the oscillations of atmospheric and LBL neutrinos in the case of the mass hierarchy (6.2). The expressions (6.9) and (6.10) describe, however, all possible oscillations between three flavour neutrinos: $\nu_{\mu} \leftrightarrows \nu_{e}, \nu_{\mu} \leftrightarrows \nu_{\tau}$ and $\nu_{e} \leftrightarrows \nu_{\tau}$.

The unitarity of the mixing matrix implies that $\sum_{\alpha}\left|U_{\alpha 3}\right|^{2}=1$ and LBL and atmospheric neutrino oscillations in the scheme under consideration are described by only three parameters: $\Delta m_{31}^{2},\left|U_{e 3}\right|^{2}$ and $\left|U_{\mu 3}\right|^{2}$.

Let us also stress that the transition probabilities (6.7) are independent on the phases of the mixing matrix $U$. Thus, in the case of a hierarchy of neutrino masses the relation (3.18) is automatically satisfied and effects of CP violation in the lepton sector cannot be revealed in neutrino oscillation experiments.

As it is seen from Eq.(6.8), the $\bar{\nu}_{e}$ survival probability in reactor LBL experiments depends on the parameters $\Delta m_{31}^{2}$ and $\left|U_{e 3}\right|^{2}$. This fact allows to obtain information on the value of $\left|U_{e 3}\right|^{2}$ in LBL reactor experiments and, in particular, from the results of the first of them, CHOOZ [159], in which no indications in favour of neutrino oscillations were found. The exclusion curve in the plane of the two-neutrino mixing parameters $\sin ^{2} 2 \vartheta$ and $\Delta m^{2}$ that was obtained in the CHOOZ experiment is shown in Fig. 3.3 .

Comparing Eqs.(3.21) and (6.8), one can see that in the three-neutrino scheme under consideration in this section

$$
\sin ^{2} 2 \vartheta=B_{e ; e}=4\left|U_{e 3}\right|^{2}\left(1-\left|U_{e 3}\right|^{2}\right) .
$$

Therefore, we can obtain information on the parameter $\left|U_{e 3}\right|^{2}$ from the exclusion plot obtained in the CHOOZ experiment. From Eq.(6.11) we have

$$
\left|U_{e 3}\right|^{2}=\frac{1}{2}\left(1 \pm \sqrt{1-B_{e ; e}}\right),
$$

and the exclusion curves shown in Fig. 3.3 imply the constraint

$$
B_{e ; e} \leq B_{e ; e}^{0},
$$


with a value of $B_{e ; e}^{0}$ that depends on $\Delta m_{31}^{2}$. From Eqs. (6.12) and (6.13) it follows that $\left|U_{e 3}\right|^{2}$ must satisfy one of the two inequalities

$$
\left|U_{e 3}\right|^{2} \leq a_{e}^{0} \quad \text { or } \quad\left|U_{e 3}\right|^{2} \geq 1-a_{e}^{0}
$$

with

$$
a_{e}^{0}=\frac{1}{2}\left(1-\sqrt{1-B_{e ; e}^{0}}\right) .
$$

From the CHOOZ exclusion curve in Fig. 3.3 (90\% CL) we find $B_{e ; e} \leq 0.18$ for $\Delta m_{31}^{2} \gtrsim$ $2 \times 10^{-3} \mathrm{eV}^{2}$ and therefore

$$
\left|U_{e 3}\right|^{2} \leq 5 \times 10^{-2} \quad \text { or } \quad\left|U_{e 3}\right|^{2} \geq 0.95
$$

\subsubsection{Solar neutrinos}

Large values of $\left|U_{e 3}\right|^{2}$ as those implied by the second inequality in Eq.(6.16) are excluded by the data of solar neutrino experiments. In order to prove this statement, let us derive the expression for the averaged survival probability of solar electron neutrinos in the case of vacuum oscillations. From Eq.(3.12) with $n=3$, taking into account that for solar neutrinos $\Delta m_{31}^{2} L / E \gg 1$, the survival probability averaged over the unobservable fast oscillations due to $\Delta m_{31}^{2}$ is given by

$$
P_{\nu_{e} \rightarrow \nu_{e}}^{(\operatorname{sun})}=\left.|| U_{e 1}\right|^{2}+\left.\left|U_{e 2}\right|^{2} \exp \left(-i \frac{\Delta m_{21}^{2} L}{2 E}\right)\right|^{2}+\left|U_{e 3}\right|^{4}
$$

This expression can be rewritten in the form

$$
P_{\nu_{e} \rightarrow \nu_{e}}^{(\operatorname{sun})}=\left(1-\left|U_{e 3}\right|^{2}\right)^{2} P_{\nu_{e} \rightarrow \nu_{e}}^{(1,2)}+\left|U_{e 3}\right|^{4}
$$

where $P_{\nu_{e} \rightarrow \nu_{e}}^{(1,2)}$ is the two-generation survival probability

$$
P_{\nu_{e} \rightarrow \nu_{e}}^{(1,2)}=1-\frac{1}{2} \sin ^{2} 2 \vartheta_{\text {sun }}\left(1-\cos \frac{\Delta m_{\mathrm{sun}}^{2} L}{2 E}\right)
$$

(see Eq.(3.21)) with $\Delta m_{\text {sun }}^{2}=\Delta m_{21}^{2}$ and the mixing angle $\vartheta_{\text {sun }}$ determined by

$$
\cos ^{2} \vartheta_{\text {sun }}=\frac{\left|U_{e 1}\right|^{2}}{1-\left|U_{e 3}\right|^{2}}, \quad \sin ^{2} \vartheta_{\text {sun }}=\frac{\left|U_{e 2}\right|^{2}}{1-\left|U_{e 3}\right|^{2}} .
$$

The relation (6.18) is also valid if matter effects are taken into account (see Eq.(4.48) and Ref. 406). In this case, $P_{\nu_{e} \rightarrow \nu_{e}}^{(1,2)}$ is the two-neutrino survival probability in matter given in Eq.(4.41), with $\Delta m^{2}$ replaced by $\Delta m_{21}^{2}$ and $\vartheta$ by $\vartheta_{\text {sun }}$.

Considering now the constraints (6.16) on $\left|U_{e 3}\right|^{2}$, if $\left|U_{e 3}\right|^{2}$ is large $\left(\left|U_{e 3}\right|^{2} \geq 0.95\right)$, from Eq. 6.18) one can see that $P_{\nu_{e} \rightarrow \nu_{e}}^{(\operatorname{sun}} \geq 0.90$. Such a large value of $P_{\nu_{e} \rightarrow \nu_{e}}$ is incompatible with the deficit of solar $\nu_{e}$ 's observed in all solar neutrino experiments. Therefore, from 
the results of the CHOOZ experiment we reach the conclusion that the element $\left|U_{e 3}\right|^{2}$ is small:

$$
\left|U_{e 3}\right|^{2} \leq 5 \times 10^{-2} \quad \text { for } \quad \Delta m_{31}^{2} \gtrsim 2 \times 10^{-3} \mathrm{eV}^{2} .
$$

This limit implies that

$$
P_{\nu_{e} \rightarrow \nu_{e}}^{(\operatorname{sun})} \simeq P_{\nu_{e} \rightarrow \nu_{e}}^{(1,2)} \quad \text { and } \quad \sin ^{2} 2 \vartheta_{\text {sun }} \simeq 4\left|U_{e 2}\right|^{2}\left(1-\left|U_{e 2}\right|^{2}\right)
$$

Hence, the oscillations of solar neutrinos are practically independent of the value of $\left|U_{e 3}\right|^{2}$ and are decoupled from the oscillations of atmospheric and LBL neutrinos, which depend only on the elements $U_{\alpha 3}$ of the mixing matrix (see Eqs.66.9) and (6.10)). This means that the two-generation analyses of the solar neutrino data are appropriate also in the three-neutrino scheme and they give information on the values of $\Delta m_{21}^{2}=\Delta m_{\text {sun }}^{2}$, $\left|U_{e 1}\right| \simeq \cos \vartheta_{\text {sun }}$ and $\left|U_{e 2}\right| \simeq \sin \vartheta_{\text {sun }} 407$.

According to a recent analysis of the solar neutrino data [344], which include preliminary data from Super-Kamiokande 46, 408, the ranges of the mixing parameters allowed at 90\% CL for the small and large mixing angle MSW solutions and for the vacuum oscillation solution are, respectively,

$$
\begin{array}{rlrl}
4 \times 10^{-6} \mathrm{eV}^{2} & \lesssim \Delta m_{\text {sun }}^{2} \lesssim 1.2 \times 10^{-5} \mathrm{eV}^{2}, & & 3 \times 10^{-3} \lesssim \sin ^{2} 2 \vartheta_{\text {sun }} \lesssim 1.1 \times 10^{-2} \\
8 \times 10^{-6} \mathrm{eV}^{2} \lesssim \Delta m_{\text {sun }}^{2} \lesssim 3.0 \times 10^{-5} \mathrm{eV}^{2}, & & 0.42 \lesssim \sin ^{2} 2 \vartheta_{\text {sun }} \lesssim 0.74 \\
6 \times 10^{-11} \mathrm{eV}^{2} \lesssim \Delta m_{\text {sun }}^{2} \lesssim 1.1 \times 10^{-10} \mathrm{eV}^{2}, & & 0.70 \lesssim \sin ^{2} 2 \vartheta_{\text {sun }} \leq 1
\end{array}
$$

Therefore, taking into account that $\left|U_{e 3}\right|^{2}$ is small and assuming that $\left|U_{e 2}\right| \leq\left|U_{e 1}\right|$ (this choice is necessary only for the MSW solutions in order to fulfill the resonance condition (4.34)), we have

$$
\begin{aligned}
\left|U_{e 1}\right| \simeq 1, & \left|U_{e 2}\right| \simeq 0.03-0.05 & & \text { (small mixing MSW) } \\
\left|U_{e 1}\right| \simeq 0.87-0.94, & \left|U_{e 2}\right| \simeq 0.35-0.49 & & \text { (large mixing MSW) } \\
\left|U_{e 1}\right| \simeq 0.71-0.88, & \left|U_{e 2}\right| \simeq 0.48-0.71 & & \text { (vacuum oscillations) } .
\end{aligned}
$$

Notice that these ranges are statistically rather stable. For example, the range of $\sin ^{2} 2 \vartheta_{\text {sun }}$ allowed at $99 \% \mathrm{CL}$ in the case of the large mixing angle MSW solution is $0.36 \lesssim \sin ^{2} 2 \vartheta_{\text {sun }} \lesssim 0.85$ 344, which implies $\left|U_{e 1}\right| \simeq 0.83-0.95,\left|U_{e 2}\right| \simeq 0.32-0.55$ (compare with Eq.(6.27)).

\subsubsection{Atmospheric neutrinos}

The atmospheric neutrino data of the Super-Kamiokande experiment give an additional indication that $\left|U_{e 3}\right|^{2}$ is small. Indeed, the up-down asymmetry of electron events $A_{e}$ that could be generated by $\nu_{\mu} \rightarrow \nu_{e}$ or $\nu_{e} \rightarrow \nu_{\beta}$ (with $\beta \neq e$ ) transitions is compatible with zero (see Section 5.1.3) [2]:

$$
A_{e}=-0.036 \pm 0.067 \pm 0.02 \text {. }
$$


In the three-neutrino scheme under consideration, the Super-Kamiokande atmospheric neutrino data can be explained by $\nu_{\mu} \rightarrow \nu_{\tau}$ oscillations [2, 261, 409]. The analysis of the data under the assumption of two-generation mixing yields the following constraints:

$$
A_{\mu ; \tau} \equiv \sin ^{2} 2 \vartheta_{\text {atm }} \gtrsim 0.82, \quad 5 \times 10^{-4} \mathrm{eV}^{2} \lesssim \Delta m^{2} \lesssim 6 \times 10^{-3} \mathrm{eV}^{2},
$$

from which it follows with Eqs. 6.7) and (6.9) that $\left|U_{\mu 3}\right|^{2}$ and $\left|U_{\tau 3}\right|^{2}$ must be close to 0.5 and therefore $\left|U_{e 3}\right|^{2}$ small.

Coming back to Eq.(6.21) which implies that $\left|U_{e 3}\right| \leq 0.22$ we see that the CHOOZ upper bound for $\left|U_{e 3}\right|$ is not very small. It is suggestive, however, to assume that

$$
\left|U_{e 3}\right| \ll 1
$$

i.e. that the element $\left|U_{e 3}\right|$ is so small that it is unimportant for neutrino oscillations 407,410. Such an assumption has been recently adopted by several authors 411].

Let us infer some consequences of the assumption (6.31). First of all, it is easy to see that if the inequality (6.31) is satisfied, matter effects are not essential for $\nu_{\mu} \rightarrow$ $\nu_{\tau}$ transitions of atmospheric and LBL neutrinos. Indeed, the evolution equation for neutrinos in matter in the flavour representation is (see Section 4)

$$
i \frac{\mathrm{d}}{\mathrm{d} x} \psi=\frac{1}{2 E}\left(U \Delta M^{2} U^{\dagger}+\hat{A}\right) \psi
$$

with

$$
\psi \equiv\left(\begin{array}{l}
a_{e} \\
a_{\mu} \\
a_{\tau}
\end{array}\right), \quad \Delta M^{2} \equiv \operatorname{diag}\left(0, \Delta m_{21}^{2}, \Delta m_{31}^{2}\right), \quad \hat{A} \equiv \operatorname{diag}\left(A_{C C}, 0,0\right)
$$

and

$$
A_{C C} \equiv 2 E V_{C C}, \quad V_{C C}=\sqrt{2} G_{F} N_{e} .
$$

Remember that for anti-neutrinos the matter potential term $A_{C C}$ must be replaced by $\bar{A}_{C C}=-A_{C C}$.

The inequality (6.5) implies that the phase generated by $\Delta m_{21}^{2}$ can be neglected for atmospheric neutrinos and $\Delta M^{2}$ can be approximated by

$$
\Delta M^{2} \simeq \operatorname{diag}\left(0,0, \Delta m_{31}^{2}\right) .
$$

In this case (taking into account that the phases of the matrix elements $U_{\alpha 3}$ can be included in the charged lepton fields) we have

$$
\left(U \Delta M^{2} U^{\dagger}\right)_{\alpha \beta} \simeq \Delta m_{31}^{2}\left|U_{\alpha 3}\right|\left|U_{\beta 3}\right|
$$

If the condition (6.31) is satisfied, the elements of the matrix $U \Delta M^{2} U^{\dagger}$ with indices $\alpha=e$ or/and $\beta=e$ can be neglected. This means that the equation for the wave function $a_{e}$ is decoupled from those of $a_{\mu}, a_{\tau}$ :

$$
\begin{aligned}
i \frac{\mathrm{d} a_{e}}{\mathrm{~d} x} & =\frac{A_{C C}}{2 E} a_{e}, \\
i \frac{\mathrm{d} a_{\alpha}}{\mathrm{d} x} & =\frac{\Delta m_{31}^{2}}{2 E}\left|U_{\alpha 3}\right| \sum_{\beta=\mu, \tau}\left|U_{\beta 3}\right| a_{\beta} \quad(\alpha=\mu, \tau) .
\end{aligned}
$$


These equations imply that $P_{\nu_{e} \rightarrow \nu_{e}}^{(\text {atm, LBL })}=1$ and $P_{\nu_{\mu} \rightarrow \nu_{\tau}}^{(\text {atm,LBL })}$ is given by the two-generation expression for neutrino oscillations in vacuum,

$$
P_{\nu_{\mu} \rightarrow \nu_{\tau}}^{(\mathrm{atm}, \mathrm{LBL})}=\frac{1}{2} \sin ^{2} 2 \vartheta_{\mathrm{atm}}\left(1-\cos \frac{\Delta m_{\mathrm{atm}}^{2} L}{2 E}\right)
$$

(see Eq. $(3.20)$ ), with $\Delta m_{\text {atm }}^{2}=\Delta m_{31}^{2}$ and the mixing angle $\vartheta_{\text {atm }}$ determined by

$$
\sin ^{2} \vartheta_{\mathrm{atm}}=\left|U_{\mu 3}\right|^{2}, \quad \cos ^{2} \vartheta_{\mathrm{atm}}=\left|U_{\tau 3}\right|^{2} .
$$

Therefore, if the condition (6.31) is satisfied, there are no $\nu_{\mu} \leftrightarrows \nu_{e}$ oscillations in atmospheric and LBL neutrino experiments and matter effects are not important for $\nu_{\mu} \rightarrow \nu_{\tau}$ transitions. This means that the two-generation analyses of the atmospheric neutrino data in terms of $\nu_{\mu} \rightarrow \nu_{\tau}$ are appropriate in the three-neutrino scheme under consideration and yield information on the values of the parameters $\Delta m_{31}^{2}=\Delta m_{\mathrm{atm}}^{2}$ and $\left|U_{\mu 3}\right|=\sin \vartheta_{\text {atm }}$.

According to a recent analysis [409] of the atmospheric neutrino data, the ranges of $\Delta m_{\mathrm{atm}}^{2}$ and $\sin ^{2} 2 \vartheta_{\mathrm{atm}}$ for $\nu_{\mu} \rightarrow \nu_{\tau}$ oscillations allowed at $90 \%$ CL by the SuperKamiokande multi-GeV data and by the data of atmospheric neutrino oscillation experiments are, respectively,

$$
\begin{array}{lll}
4 \times 10^{-4} \mathrm{eV}^{2} \lesssim \Delta m_{\text {atm }}^{2} \lesssim 8 \times 10^{-3} \mathrm{eV}^{2}, & & 0.72 \lesssim \sin ^{2} 2 \vartheta_{\text {atm }} \leq 1, \\
4 \times 10^{-4} \mathrm{eV}^{2} \lesssim \Delta m_{\text {atm }}^{2} \lesssim 6 \times 10^{-3} \mathrm{eV}^{2}, & & 0.76 \lesssim \sin ^{2} 2 \vartheta_{\text {atm }} \leq 1 .
\end{array}
$$

Taking into account the comments after Eq.(6.5), the ranges (6.42) of the two-generation mixing parameters can be used in order to constrain the values of $\left|U_{\mu 3}\right|$ and $\left|U_{\tau 3}\right|$ only in the case of the vacuum oscillation solution of the solar neutrino problem. If the solar neutrino problem is due to MSW transitions, we must use the less restrictive ranges (6.41). Thus, assuming that $\left|U_{\mu 3}\right| \leq\left|U_{\tau 3}\right|$, we have

$$
\begin{array}{rlrl}
\left|U_{\mu 3}\right| & \simeq 0.49-0.71, \quad\left|U_{\tau 3}\right| \simeq 0.71-0.87 & & \text { (solar MSW) }, \\
\left|U_{\mu 3}\right| & \simeq 0.51-0.71, \quad\left|U_{\tau 3}\right| \simeq 0.71-0.86 & \text { (solar vacuum oscillations) } .
\end{array}
$$

As in the case of the ranges (6.26)-(6.28), also the ranges (6.43)-(6.44) are statistically rather stable. For example, the range of $\sin ^{2} 2 \vartheta_{\text {sun }}$ allowed at $99 \%$ CL by all atmospheric neutrino data is $0.66 \lesssim \sin ^{2} 2 \vartheta_{\text {sun }} \leq 1$ 409, which implies $\left|U_{\mu 3}\right| \simeq 0.46-0.71,\left|U_{\tau 3}\right| \simeq$ $0.71-0.89$ (compare with Eq.(6.44)).

\subsubsection{The mixing matrix in the case $\left|U_{e 3}\right| \ll 1$}

We have seen that, if we assume that $\left|U_{e 3}\right|$ is very small, the results of the two-generation analyses of the data of solar and atmospheric neutrino experiments allow to determine the moduli of the elements of the first line and of the third column of the mixing matrix $U$. The moduli of the other elements can be determined from the unitarity of $U$. The simplest way to do it is to start from the parameterization (2.20) of the $3 \times 3$ mixing matrix $U$. 
A very small $\left|U_{e 3}\right|$ implies that $\left|s_{13}\right| \ll 1$. Since in the parameterization $(2.20)$ the CP-violating phase $\delta_{13}$ is associated with $s_{13}$, it follows that CP violation is negligible in the lepton sector (see also footnote 5) and we have

$$
U \simeq\left(\begin{array}{ccc}
c_{12} & s_{12} & \ll 1 \\
-s_{12} c_{23} & c_{12} c_{23} & s_{23} \\
s_{12} s_{23} & -c_{12} s_{23} & c_{23}
\end{array}\right)
$$

Using the information on $\left|s_{12}\right| \simeq\left|U_{e 2}\right|$ and $\left|s_{23}\right| \simeq\left|U_{\mu 3}\right|$ given by the two-generation analyses of the results of solar and atmospheric neutrino experiments, we obtain for the moduli of the elements of the mixing matrix 407]:

$$
\begin{array}{ll}
\text { Small mixing MSW: } & \left(\begin{array}{ccc}
\simeq 1 & 0.03-0.05 & \ll 1 \\
0.02-0.05 & 0.71-0.87 & 0.49-0.71 \\
0.01-0.04 & 0.48-0.71 & 0.71-0.87
\end{array}\right), \\
\text { Large mixing MSW: } & \left(\begin{array}{ccc}
0.87-0.94 & 0.35-0.49 & \ll 1 \\
0.25-0.43 & 0.61-0.82 & 0.49-0.71 \\
0.17-0.35 & 0.42-0.66 & 0.71-0.87
\end{array}\right), \\
\text { Vacuum oscillations: } & \left(\begin{array}{ccc}
0.71-0.88 & 0.48-0.71 & \ll 1 \\
0.34-0.61 & 0.50-0.76 & 0.51-0.71 \\
0.24-0.50 & 0.36-0.62 & 0.71-0.86
\end{array}\right) .
\end{array}
$$

In the case of the small mixing angle MSW solution of the solar neutrino problem $\left|U_{e 3}\right| \ll$ 1 could be of the same order of magnitude as $\left|U_{e 2}\right|$.

The best fit of Super-Kamiokande data corresponds to maximal mixing, $\vartheta_{23}=\pi / 4$ and the vacuum oscillation solution of the solar neutrino problem includes the maximal mixing case $\vartheta_{12}=\pi / 4$. The combination of these two possibilities gives the bi-maximal mixing matrix

$$
U=\left(\begin{array}{rrc}
\frac{1}{\sqrt{2}} & \frac{1}{\sqrt{2}} & 0 \\
-\frac{1}{2} & \frac{1}{2} & \frac{1}{\sqrt{2}} \\
\frac{1}{2} & -\frac{1}{2} & \frac{1}{\sqrt{2}}
\end{array}\right)
$$

that has been assumed recently by several authors [411]. Notice that bi-maximal mixing is not compatible with the large mixing angle MSW solution of the solar neutrino problem 412 .

It is interesting to note that, because of the large mixing of $\nu_{\mu}$ and $\nu_{\tau}$ with $\nu_{2}$, the transitions of solar $\nu_{e}$ 's in $\nu_{\mu}$ 's and $\nu_{\tau}$ 's are of comparable magnitude. However, this phenomenon and the values of the entries in the $\left(\nu_{\mu}, \nu_{\tau}\right)-\left(\nu_{1}, \nu_{2}\right)$ sector of the mixing matrix cannot be checked with solar neutrino experiments because the low-energy $\nu_{\mu}$ 's and $\nu_{\tau}$ 's coming from the sun can be detected only with neutral-current interactions, which are flavour-blind. Moreover, it will be very difficult to check the values of $\left|U_{\mu 1}\right|$, $\left|U_{\mu 2}\right|,\left|U_{\tau 1}\right|$ and $\left|U_{\tau 2}\right|$ in laboratory experiments because of the smallness of $\Delta m_{21}^{2}$.

In the derivation of Eqs.(6.46) -(6.48) we have assumed that $\left|U_{e 2}\right| \leq\left|U_{e 1}\right|$ and $\left|U_{\mu 3}\right| \leq$ $\left|U_{\tau 3}\right|$. The other possibilities, $\left|U_{e 2}\right| \geq\left|U_{e 1}\right|$ and $\left|U_{\mu 3}\right| \geq\left|U_{\tau 3}\right|$, are equivalent, respectively, 
to an exchange of the first and second columns and to an exchange of the second and third rows in the matrices (6.46)-(6.48). Unfortunately, these alternatives are hard to distinguish experimentally because of the above mentioned difficulty to measure directly the values of $\left|U_{\mu 1}\right|,\left|U_{\mu 2}\right|,\left|U_{\tau 1}\right|$ and $\left|U_{\tau 2}\right|$. Only the choice $\left|U_{e 2}\right| \leq\left|U_{e 1}\right|$, which is necessary for the MSW solutions of the solar neutrino problem, could be confirmed by the results of the new generation of solar neutrino experiments (Super-Kamiokande, SNO, ICARUS, Borexino, GNO and others [353]) if they will allow to exclude the vacuum oscillation solution.

\subsubsection{Accelerator long-baseline experiments}

Future results from reactor long-baseline neutrino oscillation experiments (CHOOZ 413], Palo Verde [288], Kam-Land [290]) could allow to improve the upper bound (6.21) on $\left|U_{e 3}\right|^{2}$. In this section we discuss how an improvement of this upper bound could be obtained by future accelerator long-baseline neutrino oscillation experiments that are sensitive to $\nu_{\mu} \rightarrow \nu_{e}$ transitions (K2K [271], MINOS [293], ICARUS 294] and others 295 298]; for a review see Ref. [414]).

If matter effects are not important, in the scheme under consideration the parameter $\sin ^{2} 2 \vartheta_{\mu e}$ measured in $\nu_{\mu} \rightarrow \nu_{e}$ long-baseline experiments is given by (see Eq.(6.9))

$$
\sin ^{2} 2 \vartheta_{\mu e}=4\left|U_{e 3}\right|^{2}\left|U_{\mu 3}\right|^{2}
$$

If accelerator long-baseline neutrino oscillation experiments will not observe $\nu_{\mu} \rightarrow \nu_{e}$ transitions and will place an upper bound $\sin ^{2} 2 \vartheta_{\mu e} \leq \sin ^{2} 2 \vartheta_{\mu e}^{(\max )}$, it will be possible to obtain the limit

$$
\left|U_{e 3}\right|^{2} \leq \frac{\sin ^{2} 2 \vartheta_{\mu e}^{(\max )}}{4\left|U_{\mu 3}\right|_{(\min )}^{2}}
$$

where $\left|U_{\mu 3}\right|_{\text {(min) }}^{2}$ is the minimum value of $\left|U_{\mu 3}\right|^{2}$ allowed by the solution of the atmospheric neutrino anomaly and by the observation of $\nu_{\mu} \rightarrow \nu_{\tau}$ long-baseline transitions. For example, taking $\left|U_{\mu 3}\right|_{(\min )}^{2}=0.25$ (see Eq.(6.44)) we have $\left|U_{e 3}\right|^{2} \leq \sin ^{2} 2 \vartheta_{\mu e}^{(\max )}$. If a value of $\sin ^{2} 2 \vartheta_{\mu e}^{(\max )} \simeq 10^{-3}$, corresponding to the sensitivity of the ICARUS experiment for one year of running [294, will be reached, it will be possible to put the upper bound $\left|U_{e 3}\right| \lesssim 3 \times 10^{-2}$

The observation of $\nu_{\mu} \rightarrow \nu_{\tau}$ transitions in long-baseline experiments will allow to establish a lower bound for $\left|U_{\mu 3}\right|^{2}$ because the parameter $\sin ^{2} 2 \vartheta_{\mu \tau}$ is given in the scheme under consideration by (see Eq. (6.9))

$$
\sin ^{2} 2 \vartheta_{\mu \tau}=4\left|U_{\mu 3}\right|^{2}\left|U_{\tau 3}\right|^{2} .
$$

From the unitarity relation $\left|U_{e 3}\right|^{2}+\left|U_{\mu 3}\right|^{2}+\left|U_{\tau 3}\right|^{2}=1$ it follows that an experimental lower bound $\sin ^{2} 2 \vartheta_{\mu \tau} \geq \sin ^{2} 2 \vartheta_{\mu \tau}^{(\min )}$ allows to constrain $\left|U_{\mu 3}\right|^{2}$ in the range

$$
\frac{1}{2}\left(1-\sqrt{1-\sin ^{2} 2 \vartheta_{\mu \tau}^{(\min )}}\right) \leq\left|U_{\mu 3}\right|^{2} \leq \frac{1}{2}\left(1+\sqrt{1-\sin ^{2} 2 \vartheta_{\mu \tau}^{(\min )}}\right)
$$


If $\sin ^{2} 2 \vartheta_{\mu \tau}^{(\min )}$ is found to be close to one, as suggested by the solution of the atmospheric neutrino problem (see Eqs.6.41) and (6.42)), the lower bound $\left|U_{\mu 3}\right|_{(\min )}^{2}=$ $\frac{1}{2}\left(1-\sqrt{1-\sin ^{2} 2 \vartheta_{\mu \tau}^{(\min )}}\right)$ is close to $1 / 2$.

If matter effects are important, the extraction of an upper bound for $\left|U_{e 3}\right|^{2}$ from the data of $\nu_{\mu} \rightarrow \nu_{e}$ accelerator long-baseline experiments is more complicated. In this case the probability of $\nu_{\mu} \rightarrow \nu_{e}$ oscillations is given by 192

$$
P_{\nu_{\mu} \rightarrow \nu_{e}}=\frac{4\left|U_{e 3}\right|^{2}\left|U_{\mu 3}\right|^{2}}{\left(1-\frac{A_{C C}}{\Delta m_{31}^{2}}\right)^{2}+4\left|U_{e 3}\right|^{2} \frac{A_{C C}}{\Delta m_{31}^{2}}} \sin ^{2}\left(\frac{\Delta m_{31}^{2} L}{4 E} \sqrt{\left(1-\frac{A_{C C}}{\Delta m_{31}^{2}}\right)^{2}+4\left|U_{e 3}\right|^{2} \frac{A_{C C}}{\Delta m_{31}^{2}}}\right)
$$

where $E$ is the neutrino energy and $L$ is the distance of propagation. This probability depends on the neutrino energy not only through the explicit $E$ in the denominator of the phase, but also through the energy dependence of $A_{C C} \equiv 2 E V_{C C}$. For longbaseline neutrino beams propagating in the lithosphere of the earth the charged-current effective potential $V_{C C}=\sqrt{2} G_{F} N_{e}$ is practically constant: $N_{e} \simeq 1.5 N_{\text {avo }} \mathrm{cm}^{-3}$ and $V_{C C} \simeq 1.1 \times 10^{-13} \mathrm{eV}$ (see Eq.(4.37)).

If long-baseline experiments will not observe $\nu_{\mu} \rightarrow \nu_{e}$ transitions (or will find that they have an extremely small probability) for neutrino energies such that $A_{C C} \lesssim \Delta m_{31}^{2}$, it will mean that $\left|U_{e 3}\right|^{2}$ is small and a fit of the experimental data with the formula (6.54) will yield a stringent upper limit for $\left|U_{e 3}\right|^{2}$ (taking into account the lower limit $\left|U_{\mu 3}\right|^{2} \geq\left|U_{\mu 3}\right|_{(\min )}^{2}$ obtained from the solution of the atmospheric neutrino anomaly and from the observation of $\nu_{\mu} \rightarrow \nu_{\tau}$ long-baseline transitions). On the other hand, the nonobservation of $\nu_{\mu} \rightarrow \nu_{e}$ transitions for neutrino energies such that $A_{C C} \gg \Delta m_{31}^{2}$ does not provide any information on $\left|U_{e 3}\right|^{2}$ because in this case the transition probability (6.54) is suppressed by the matter effect. Hence, in order to check the hypothesis $\left|U_{e 3}\right| \ll 1$, as well as to have some possibility to observe $\nu_{\mu} \rightarrow \nu_{e}$ transitions if this hypothesis is wrong, it is necessary that a substantial part of the energy spectrum of the neutrino beam lies below

$$
\frac{\Delta m_{31}^{2}}{2 V_{C C}} \simeq 30 \mathrm{GeV}\left(\frac{\Delta m_{31}^{2}}{10^{-2} \mathrm{eV}^{2}}\right)
$$

This requirement will be satisfied in the accelerator long-baseline experiments under preparation (K2K [271], MINOS [293], ICARUS [294] and others [295]298]; see [414]) if $\Delta m_{31}^{2}$ is not much smaller than $10^{-2} \mathrm{eV}^{2}$.

\subsection{Mixing of four massive neutrinos}

Let us consider now the case of mixing of four neutrinos 415 417, i.e., $n=4$ in Eqs.(3.3) and (3.9),

$$
\left|\nu_{\alpha}\right\rangle=\sum_{k=1}^{4} U_{\alpha k}^{*}\left|\nu_{k}\right\rangle, \quad\left|\bar{\nu}_{\alpha}\right\rangle=\sum_{k=1}^{4} U_{\alpha k}\left|\bar{\nu}_{k}\right\rangle \quad(\alpha=e, \mu, \tau, s)
$$




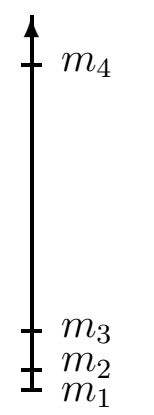

(I)

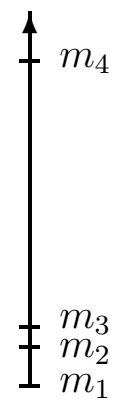

(II)

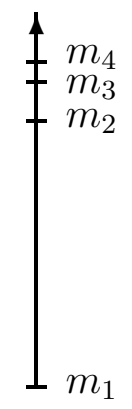

(III)

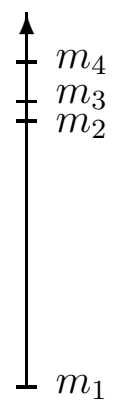

(IV)

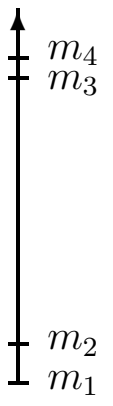

(A)

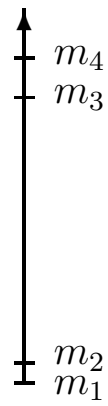

(B)

Figure 6.1. The six types of neutrino mass spectra that can accommodate the solar, atmospheric and LSND scales of $\Delta m^{2}$.

which allows to accommodate all the three existing indications in favour of neutrino mixing. Here $\left|\nu_{s}\right\rangle$ is a sterile neutrino state (see Section 2.2).

We do not make any assumptions about the mass spectrum of four neutrinos and will consider all possibilities. The six possible four-neutrino mass spectra with three different scales of neutrino mass-squared differences (atmospheric, solar and LSND) are shown in Fig. 6.1. In all these mass spectra there are two groups of close masses separated by the "LSND gap" of the order of $1 \mathrm{eV}$. The small mass-squared differences correspond to $\Delta m_{\text {sun }}^{2}$ (the smallest one, $\Delta m_{21}^{2}$ in schemes I and $\mathrm{B}, \Delta m_{32}^{2}$ in schemes II and IV, $\Delta m_{43}^{2}$ in schemes III and A) and $\Delta m_{\text {atm }}^{2}\left(\Delta m_{31}^{2}\right.$ in schemes I and II, $\Delta m_{42}^{2}$ in schemes III and IV, $\Delta m_{21}^{2}$ in scheme A, $\Delta m_{43}^{2}$ in scheme B) and the largest mass squared difference $\Delta m_{41}^{2}=\Delta m_{\mathrm{LSND}}^{2}$ is relevant for the oscillations observed in the LSND experiment.

Let us consider SBL oscillations in the case of neutrino mass spectra presented in Fig. 6.1. Taking into account that

$$
\frac{\Delta m_{\mathrm{sun}}^{2} L}{E} \ll 1 \quad \text { and } \quad \frac{\Delta m_{\mathrm{atm}}^{2} L}{E} \ll 1,
$$

from the general formula (3.12) we obtain that the probabilities of $\nu_{\alpha} \rightarrow \nu_{\beta}$ transitions and of $\nu_{\alpha}$ survival in SBL experiments are given by

$$
\begin{aligned}
& P_{\nu_{\alpha} \rightarrow \nu_{\beta}}^{(\mathrm{SBL})}=\frac{1}{2} A_{\alpha ; \beta}\left(1-\cos \frac{\Delta m_{41}^{2} L}{2 E}\right) \quad(\alpha \neq \beta), \\
& P_{\nu_{\alpha} \rightarrow \nu_{\alpha}}^{(\mathrm{SBL})}=1-\frac{1}{2} B_{\alpha ; \alpha}\left(1-\cos \frac{\Delta m_{41}^{2} L}{2 E}\right) .
\end{aligned}
$$

The oscillation amplitudes $A_{\alpha ; \beta}$ and $B_{\alpha ; \alpha}$ depend on the elements of the mixing matrix and on the type of neutrino mass spectrum:

$$
\begin{aligned}
& A_{\alpha ; \beta}=4\left|\sum_{k} U_{\alpha k}^{*} U_{\beta k}\right|^{2}, \\
& B_{\alpha ; \alpha}=4\left(\sum_{k}\left|U_{\alpha k}\right|^{2}\right)\left(1-\sum_{k}\left|U_{\alpha k}\right|^{2}\right),
\end{aligned}
$$


where the index $k$ runs over the indices of the first or (because of the unitarity of the mixing matrix) of the second group of neutrino masses (see Fig. 6.1). The expressions (6.58) and (6.59) have the same form as the corresponding two-neutrino formulas (3.20) and (3.21) and are a direct generalization of the expressions (6.7) and (6.8) presented in Section 6.1 for the case of three massive neutrinos and a neutrino mass hierarchy. From Eq. (6.60) it is obvious that the amplitude of $\bar{\nu}_{\alpha} \rightarrow \bar{\nu}_{\beta}$ oscillations is equal to $A_{\alpha ; \beta}$ and

$$
P_{\bar{\nu}_{\alpha} \rightarrow \bar{\nu}_{\beta}}^{(\mathrm{SBL})}=P_{\nu_{\alpha} \rightarrow \nu_{\beta}}^{(\mathrm{SBL})} \text {. }
$$

Thus, CP violation in the lepton sector cannot be revealed in SBL neutrino oscillation experiments if only one mass-squared difference is relevant for SBL oscillations.

The expression (6.61) allows to obtain information on the quantities $\sum_{k}\left|U_{e k}\right|^{2}$ and $\sum_{k}\left|U_{\mu k}\right|^{2}$ from the results of SBL $\bar{\nu}_{e}$ and $\nu_{\mu}$ disappearance experiments, whose exclusion curves imply the constraint

$$
B_{\alpha ; \alpha} \leq B_{\alpha ; \alpha}^{0} \quad(\alpha=e, \mu) .
$$

The upper bound $B_{\alpha ; \alpha}^{0}$ depends on the value of $\Delta m_{41}^{2}$. Equations (6.59) and (6.63) imply that each $\sum_{k}\left|U_{\alpha k}\right|^{2}$ for $\alpha=e, \mu$ must satisfy one of the two inequalities

$$
\sum_{k}\left|U_{\alpha k}\right|^{2} \leq a_{\alpha}^{0} \quad \text { or } \quad \sum_{k}\left|U_{\alpha k}\right|^{2} \geq 1-a_{\alpha}^{0}
$$

with

$$
a_{\alpha}^{0}=\frac{1}{2}\left(1-\sqrt{1-B_{\alpha ; \alpha}^{0}}\right) .
$$

The most stringent limits on $B_{e ; e}$ and $B_{\mu ; \mu}$ are given by the exclusion curves of the Bugey [162 reactor $\bar{\nu}_{e} \rightarrow \bar{\nu}_{e}$ experiment and of the CDHS |153 and CCFR |418 accelerator $\nu_{\mu} \rightarrow \nu_{\mu}$ experiments. The values of $a_{e}^{0}$ and $a_{\mu}^{0}$ corresponding to the $90 \% \mathrm{CL}$ exclusion curves of these experiments are shown in Fig. 6.2. One can see that

$$
\begin{array}{lll}
a_{e}^{0} \leq 4 \times 10^{-2} & \text { for } & \Delta m_{41}^{2} \gtrsim 0.1 \mathrm{eV}^{2}, \\
a_{\mu}^{0} \leq 2 \times 10^{-1} & \text { for } & \Delta m_{41}^{2} \gtrsim 0.3 \mathrm{eV}^{2} .
\end{array}
$$

Hence, from Eq.(6.64) it follows that the quantities $\sum_{k}\left|U_{e k}\right|^{2}$ and $\sum_{k}\left|U_{\mu k}\right|^{2}$ are either small or large (close to one). We will show now that from the four possibilities for the two quantities $\sum_{k}\left|U_{e k}\right|^{2}$ and $\sum_{k}\left|U_{\mu k}\right|^{2}$ (small-small, small-large, large-small, large-large) for each neutrino mass spectrum in Fig. 6.1 only one possibility is compatible with the results of solar and atmospheric neutrino experiments [416, 419, 420].

Let us consider, for example, the hierarchical mass spectrum

$$
m_{1} \ll m_{2} \ll m_{3} \ll m_{4},
$$

which corresponds to type I in Fig. 6.1. In this case, the inequalities (6.64) become

$$
\left|U_{\alpha 4}\right|^{2} \leq a_{\alpha}^{0} \quad \text { or } \quad\left|U_{\alpha 4}\right|^{2} \geq 1-a_{\alpha}^{0} \quad(\alpha=e, \mu)
$$




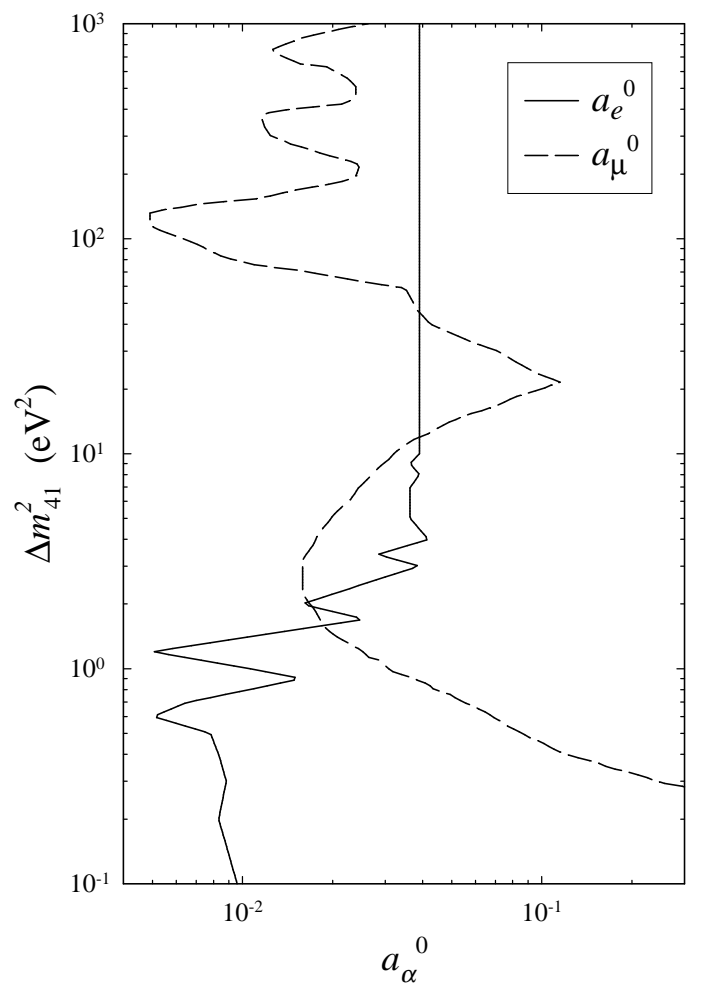

Figure 6.2. Values of $a_{e}^{0}$ and $a_{\mu}^{0}$ (see Eq.(6.65) as functions of $\Delta m_{41}^{2}$ obtained from the exclusion curves of the Bugey 162, CDHS 153] and CCFR [118] SBL disappearance experiments.

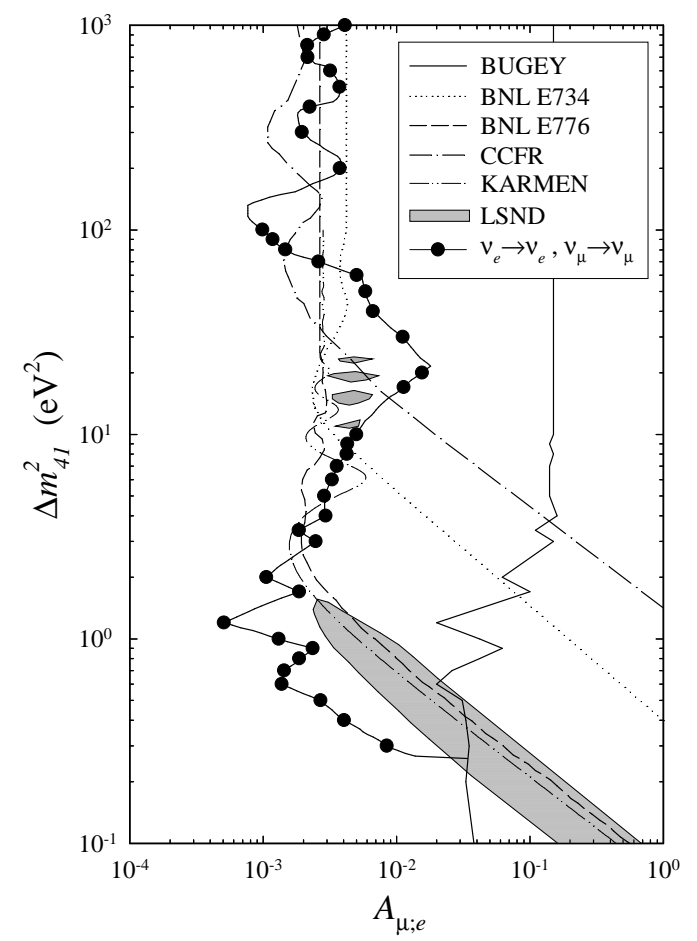

Figure 6.3. The region on the right of the solid line passing through the black circles is excluded at $90 \%$ CL by the results of SBL disappearance experiments for small $\left|U_{e 4}\right|^{2}$ and $\left|U_{\mu 4}\right|^{2}$ in the model with mixing of four neutrinos and a mass hierarchy. The shadowed regions are allowed at 90\% CL by the results of the LSND experiment. Also shown are the exclusion curves of the Bugey [162], BNL E734 [372], BNL E776 373], CCFR [374] and KARMEN (Bayesian analysis) 375] experiments.

The survival probability of solar $\nu_{e}$ 's averaged over the fast, unobservable oscillations due to $\Delta m_{41}^{2}$ and $\Delta m_{31}^{2}$ is given by 421

$$
P_{\nu_{e} \rightarrow \nu_{e}}^{(\operatorname{sun})}=\left(1-\sum_{k=3,4}\left|U_{e k}\right|^{2}\right)^{2} P_{\nu_{e} \rightarrow \nu_{e}}^{(1,2)}+\sum_{k=3,4}\left|U_{e k}\right|^{4}
$$

where $P_{\nu_{e} \rightarrow \nu_{e}}^{(1,2)}$ is the $\nu_{e}$ survival probability due to the mixing of $\nu_{e}$ with $\nu_{1}$ and $\nu_{2}$. This probability has the two-generation form (3.21) and depends on the neutrino energy, $\Delta m^{2}=\Delta m_{\text {sun }}^{2}=\Delta m_{21}^{2}$ and

$$
\sin ^{2} 2 \vartheta=\sin ^{2} 2 \vartheta_{\text {sun }}=\frac{4\left|U_{e 1}\right|^{2}\left|U_{e 2}\right|^{2}}{\left(\left|U_{e 1}\right|^{2}+\left|U_{e 2}\right|^{2}\right)^{2}} .
$$

Though Eqs.(6.70) and (6.71) were derived here for vacuum oscillations, the considerations in Section 4.4 show that they are also valid including matter effects. If $\left|U_{e 4}\right|^{2} \geq 1-a_{e}^{0}$, 
from Eqs.(6.66) and (6.70) one can see that $P_{\nu_{e} \rightarrow \nu_{e}}^{(\operatorname{sun})}$ practically does not depend on the neutrino energy and $P_{\nu_{e} \rightarrow \nu_{e}}^{(\mathrm{sun})} \gtrsim 0.92$ for all solar neutrino energies. This is not compatible with the solar neutrino data $329,349,422$. Therefore, we reach the conclusion that from the two possibilities (6.69) for $\left|U_{e 4}\right|^{2}$ only

$$
\left|U_{e 4}\right|^{2} \leq a_{e}^{0}
$$

is compatible with the results of solar neutrino experiments.

Let us now consider the survival probability of atmospheric $\nu_{\mu}$ 's. From the general formula (3.12) we obtain

$$
P_{\nu_{\mu} \rightarrow \nu_{\mu}}^{(\operatorname{atm})}=\left.\left|\sum_{k=1,2}\right| U_{\mu k}\right|^{2}+\left|U_{\mu 3}\right|^{2} \exp \left(-i \frac{\Delta m_{31}^{2} L}{2 E}\right)+\left.\left|U_{\mu 4}\right|^{2} \exp \left(-i \frac{\Delta m_{41}^{2} L}{2 E}\right)\right|^{2},
$$

where we have taken into account that for atmospheric neutrinos ${ }^{27} \Delta m_{21}^{2} L / E \ll 1$. The probability averaged over the fast unobservable oscillations due to $\Delta m_{41}^{2}$ is given by

$$
P_{\nu_{\mu} \rightarrow \nu_{\mu}}^{(\operatorname{atm})}=\left(\sum_{k=1,2}\left|U_{\mu k}\right|^{2}\right)^{2}+\left|U_{\mu 3}\right|^{4}+2\left(\sum_{k=1,2}\left|U_{\mu k}\right|^{2}\right)\left|U_{\mu 3}\right|^{2} \cos \left(\frac{\Delta m_{31}^{2} L}{2 E}\right)+\left|U_{\mu 4}\right|^{4} .
$$

Using the unitarity relation

$$
\sum_{k=1}^{3}\left|U_{\mu k}\right|^{2}=1-\left|U_{\mu 4}\right|^{2}
$$

the survival probability (6.74) can be written as 416, 420] (see also Section 4.4)

$$
P_{\nu_{\mu} \rightarrow \nu_{\mu}}^{(\operatorname{atm})}=\left(1-\left|U_{\mu 4}\right|^{2}\right)^{2} P_{\nu_{\mu} \rightarrow \nu_{\mu}}^{(1,2 ; 3)}+\left|U_{\mu 4}\right|^{4}
$$

where $P_{\nu_{\mu} \rightarrow \nu_{\mu}}^{(1,2 ; 3)}$ is the survival probability of atmospheric $\nu_{\mu}$ 's due to the mixing of $\nu_{\mu}$ with $\nu_{3}$ and $\nu_{2}, \nu_{1}$. This probability has the two-generation form

$$
P_{\nu_{\mu} \rightarrow \nu_{\mu}}^{(1,2 ; 3)}=1-\frac{1}{2} \sin ^{2} 2 \vartheta_{\mathrm{atm}}\left(1-\cos \frac{\Delta m_{31}^{2} L}{2 E}\right)
$$

(see Eq.(3.21)), with

$$
\sin ^{2} 2 \vartheta_{\mathrm{atm}}=\frac{4\left|U_{\mu 3}\right|^{2}\left(\left|U_{\mu 1}\right|^{2}+\left|U_{\mu 2}\right|^{2}\right)}{\left(\left|U_{\mu 1}\right|^{2}+\left|U_{\mu 2}\right|^{2}+\left|U_{\mu 3}\right|^{2}\right)^{2}}
$$

The zenith angle dependence of $\mu$-like events in atmospheric neutrino experiments is due to $P_{\nu_{\mu} \rightarrow \nu_{\mu}}^{(1,2 ; 3)}$, which is multiplied by $\left(1-\left|U_{\mu 4}\right|^{2}\right)^{2}$ in the expression (6.76) for $P_{\nu_{\mu} \rightarrow \nu_{\mu}}^{(\text {atm })}$. If

\footnotetext{
${ }^{27}$ As explained after Eq.(6.5), in the case of the MSW solutions of the solar neutrino problem this inequality and the following equations are valid only for multi-GeV neutrinos.
} 
$\left|U_{\mu 4}\right|^{2}$ is large, as given by the second inequality in Eq. (6.69), $\left|U_{\mu 4}\right|^{2} \geq 1-a_{\mu}^{0}$, we have $\left(1-\left|U_{\mu 4}\right|^{2}\right)^{2} \leq 4 \times 10^{-2}$ for $\Delta m_{41}^{2} \gtrsim 0.3 \mathrm{eV}^{2}$ (see Eq.(6.67)). A zenith-angle variation of $\mu$-like events smaller than $4 \times 10^{-2}$ is incompatible with the up-down asymmetry of multi-GeV $\mu$-like events observed by the Super-Kamiokande experiment (see Section 5.1). Therefore, from the two inequalities (6.69) for $\left|U_{\mu 4}\right|^{2}$ only the first one is compatible with the results of the atmospheric neutrino experiments:

$$
\left|U_{\mu 4}\right|^{2} \leq a_{\mu}^{0}
$$

The same conclusion can be reached for all the neutrino mass spectra in Fig. 6.1 in which one mass is separated by the other three by the LSND gap, i.e., for the spectra I, II, III, IV. Hence, in the framework of these spectra the results of SBL disappearance experiments and of solar and atmospheric neutrino experiments imply that

$$
\left|U_{e j}\right|^{2} \leq a_{e}^{0}, \quad\left|U_{\mu j}\right|^{2} \leq a_{\mu}^{0},
$$

with $j=4$ in the schemes I and II and $j=1$ in the schemes III and IV. These inequalities imply that $\stackrel{(-)}{\nu} \rightarrow \stackrel{(-)}{\nu}$ transitions in SBL experiments are strongly suppressed. Indeed, from Eqs. (6.60) and (6.80) we have

$$
A_{\mu ; e}=4\left|U_{e j}\right|^{2}\left|U_{\mu j}\right|^{2} \leq 4 a_{e}^{0} a_{\mu}^{0} .
$$

Thus, the amplitude of SBL $\stackrel{(-)}{\nu} \rightarrow \stackrel{(-)}{\nu}$ oscillations is quadratic in the small quantities $\left|U_{e j}\right|^{2}$ and $\left|U_{\mu j}\right|^{2}$. The upper bound (6.81) obtained from the values of $a_{e}^{0}$ and $a_{\mu}^{0}$ presented in Fig. 6.2 is shown in Fig. 6.3 by the solid line passing through the black circles. The solid, dotted, dashed, dash-dotted and dash-dot-dotted curves in Fig. 6.3 are the $90 \%$ CL exclusion curves obtained in the Bugey [162], BNL E734 [372], BNL E776 [373], CCFR [374] and KARMEN (Bayesian analysis) [375] experiments. The shadowed regions are allowed by the results of the LSND [370,371] experiments at 90\% CL. From this figure one can see that the result of the LSND experiment, taken together with the results of SBL disappearance experiments and solar and atmospheric neutrino experiments, disfavours the schemes I, II, III and IV of Fig. 6.1.28

Let us consider now the spectra A and B in Fig. 6.1. In this case the inequalities (6.64) apply with the index $k$ running over 1,2 or 3,4 (the two choices are equivalent because of the unitarity of the mixing matrix). We consider explicitly only the spectrum A, but all the following results are valid also for scheme B and can be obtained with the index exchange $1,2 \leftrightarrows 3,4$

In the case of spectrum A, the survival probability of solar $\nu_{e}$ 's is given by 421]

$$
P_{\nu_{e} \rightarrow \nu_{e}}^{(\operatorname{sun})}=\sum_{k=1,2}\left|U_{e k}\right|^{4}+\left(1-\sum_{k=1,2}\left|U_{e k}\right|^{2}\right)^{2} P_{\nu_{e} \rightarrow \nu_{e}}^{(3,4)}
$$

${ }^{28}$ The presence of a small allowed region in Fig. 6.3 for $0.2 \mathrm{eV}^{2} \lesssim \Delta m_{41}^{2} \lesssim 0.3 \mathrm{eV}^{2}$ is due to the fact that for $\Delta m_{41}^{2} \lesssim 0.3 \mathrm{eV}^{2}$ there are no constraints on $B_{\mu ; \mu}^{0}$ from the results of $\nu_{\mu}$ disappearance experiments. 
where $P_{\nu_{e} \rightarrow \nu_{e}}^{(3,4)}$ is the survival probability of solar $\nu_{e}$ 's due to the mixing of $\nu_{e}$ with $\nu_{3}$ and $\nu_{4}$. This probability has the two-generation form (3.21) and depends on the neutrino energy, $\Delta m^{2}=\Delta m_{\text {sun }}^{2}=\Delta m_{43}^{2}$ and

$$
\sin ^{2} 2 \vartheta=\sin ^{2} 2 \vartheta_{\text {sun }}=\frac{4\left|U_{e 3}\right|^{2}\left|U_{e 4}\right|^{2}}{\left(\left|U_{e 3}\right|^{2}+\left|U_{e 4}\right|^{2}\right)^{2}}
$$

If $\sum_{k=1,2}\left|U_{e k}\right|^{2} \geq 1-a_{e}^{0}$, i.e., the second inequality (6.64) for $\alpha=e$ and $k=1,2$ is satisfied, from Eq. (6.66) we have $\left(1-\sum_{k=1,2}\left|U_{e k}\right|^{2}\right)^{2} \leq 1.6 \times 10^{-3}$, which implies that $P_{\nu_{e} \rightarrow \nu_{e}}^{(\text {sun }} \simeq \sum_{k=1,2}\left|U_{e k}\right|^{4}$ is constant and $P_{\nu_{e} \rightarrow \nu_{e}}^{(\mathrm{sun})} \gtrsim 0.5$. Since these constraints are incompatible with the solar neutrino data, we reach the conclusion that only the first inequality (6.64) for $\alpha=e$ and $k=1,2$ is compatible with the results of solar neutrino experiments, i.e., $\sum_{k=1,2}\left|U_{e k}\right|^{2} \leq a_{e}^{0}$.

Now we consider the survival probability of atmospheric $\nu_{\mu}$ 's. In scheme A it is given by 421

$$
P_{\nu_{\mu} \rightarrow \nu_{\mu}}^{(\mathrm{atm})}=\left(1-\sum_{k=3,4}\left|U_{\mu k}\right|^{2}\right)^{2} P_{\nu_{\mu} \rightarrow \nu_{\mu}}^{(1,2)}+\left(\sum_{k=3,4}\left|U_{\mu k}\right|^{2}\right)^{2},
$$

where the probability $P_{\nu_{\mu} \rightarrow \nu_{\mu}}^{(1,2)}$ has the two-generation form (3.21) with $\Delta m^{2}=\Delta m_{\mathrm{atm}}^{2}=$ $\Delta m_{21}^{2}$ and

$$
\sin ^{2} 2 \vartheta=\sin ^{2} 2 \vartheta_{\mathrm{atm}}=\frac{4\left|U_{\mu 1}\right|^{2}\left|U_{\mu 2}\right|^{2}}{\left(\left|U_{\mu 1}\right|^{2}+\left|U_{\mu 2}\right|^{2}\right)^{2}} .
$$

If $\sum_{k=3,4}\left|U_{\mu k}\right|^{2} \geq 1-a_{\mu}^{0}$, which corresponds to the second of the two inequalities (6.64) for $\alpha=\mu$ and $k=3,4$, the upper bound (6.67) for $a_{\mu}^{0}$ implies that $P_{\nu_{\mu} \rightarrow \nu_{\mu}}^{(\mathrm{atm})}$ is practically constant and incompatible with the zenith-angle dependence of multi-GeV $\mu$-like events observed in the Super-Kamiokande experiment [2]. Hence, only the first inequality (6.64) for $\alpha=\mu$ and $k=3,4$ is compatible with the results of the Super-Kamiokande atmospheric neutrino experiments and we have $\sum_{k=3,4}\left|U_{\mu k}\right|^{2} \leq a_{\mu}^{0}$.

Summarizing, in the framework of scheme A the results of SBL disappearance experiments and of solar and atmospheric neutrino experiments imply that

$$
\sum_{k=1,2}\left|U_{e k}\right|^{2} \leq a_{e}^{0} \quad \text { and } \quad \sum_{k=3,4}\left|U_{\mu k}\right|^{2} \leq a_{\mu}^{0} \quad(\mathrm{~A})
$$

The corresponding bounds in scheme B are obtained with the exchange $1,2 \leftrightarrows 3,4$ :

$$
\sum_{k=3,4}\left|U_{e k}\right|^{2} \leq a_{e}^{0} \quad \text { and } \quad \sum_{k=1,2}\left|U_{\mu k}\right|^{2} \leq a_{\mu}^{0} \quad \text { (B) . }
$$

The constraints (6.86) and (6.87) imply the following upper bound for the amplitude of $\nu_{\mu} \rightarrow \nu_{e}$ transitions in SBL experiments:

$$
A_{\mu ; e}=4\left|\sum_{k} U_{e k} U_{\mu k}^{*}\right|^{2} \leq 4\left(\sum_{k}\left|U_{e k}\right|^{2}\right)\left(\sum_{k}\left|U_{\mu k}\right|^{2}\right) \leq 4 \operatorname{Min}\left(a_{e}^{0}, a_{\mu}^{0}\right)
$$


(here the index $k$ runs over 1,2 or over 3,4 ). This bound is linear in the small quantities $a_{e}^{0}$ and $a_{\mu}^{0}$ and turns out to be compatible with the results of the LSND experiment. Actually, this bound is worse than the unitarity bound

$$
A_{\mu ; e} \leq \operatorname{Min}\left(B_{e ; e}^{0}, B_{\mu ; \mu}^{0}\right),
$$

which causes the exclusion of the large $\sin ^{2} 2 \vartheta$ part of the LSND allowed region (see Fig. 6.3, where $B_{e ; e}^{0}$ is represented by the Bugey curve).

We want to note that the bound $a_{\mu}^{0}$ ceases to exist shortly below $\Delta m_{41}^{2}=0.3 \mathrm{eV}^{2}$ (see Fig. 6.2). On the other hand, it follows from the LSND and Bugey experiments that $\Delta m_{41}^{2} \gtrsim 0.2 \mathrm{eV}^{2}$ (see Fig. 6.3). Thus, for the small interval $0.2 \mathrm{eV}^{2} \lesssim \Delta m_{41}^{2} \lesssim 0.3 \mathrm{eV}^{2}$ our arguments leading to schemes $\mathrm{A}$ and $\mathrm{B}$ have to be considered with caution and it would be desirable to have experimental information on $B_{\mu ; \mu}$ in this interval.

Let us now discuss some consequences of the schemes $\mathrm{A}$ and $\mathrm{B}$, taking into account the constraints (6.86) and (6.87). First of all, we will show that these bounds lead to constraints on the neutrino mass measured in tritium $\beta$-decay experiments and on the effective Majorana mass that determines the probability of neutrinoless double- $\beta$ decay.

The electron spectrum in the decay ${ }^{3} \mathrm{H} \rightarrow{ }^{3} \mathrm{He}+e^{-}+\bar{\nu}_{e}$ is given by

$$
\frac{\mathrm{d} N}{\mathrm{~d} E}=C p_{e} E_{e}(Q-T) F\left(E_{e}\right) \sum_{i}\left|U_{e i}\right|^{2} \sqrt{(Q-T)^{2}-m_{i}^{2}},
$$

where $Q$ is the energy release, $p_{e}$ and $E_{e}$ are the electron momentum and energy, $T=$ $E_{e}-m_{e}, F\left(E_{e}\right)$ is the Fermi function and $C$ is a constant. Taking into account that $m_{1} \simeq m_{2} \ll m_{3} \simeq m_{4}$, the first inequality in Eq.(6.86) implies that in scheme A the effective neutrino mass determined from the measurement of the high-energy part of the $\beta$-spectrum of ${ }^{3} \mathrm{H}$ is practically equal to the "LSND mass" $m_{4}$ :

$$
m\left({ }^{3} \mathrm{H}\right) \simeq \sum_{k=3,4}\left|U_{e k}\right|^{2} m_{4}=\left(1-\sum_{k=1,2}\left|U_{e k}\right|^{2}\right) m_{4} \simeq m_{4} .
$$

If massive neutrinos are Majorana particles, the matrix element of neutrinoless double$\beta$ decay is proportional to the effective Majorana mass [95, 106] (see Appendix A)

$$
\langle m\rangle=\sum_{k=1}^{4} U_{e k}^{2} m_{k} .
$$

In the case of scheme A, the first inequality in Eq.(6.86) implies that

$$
|\langle m\rangle| \simeq\left|\sum_{k=3,4} U_{e k}^{2}\right| m_{4} \simeq m_{4} \sqrt{1-4\left|U_{e 4}\right|^{2}\left(1-\left|U_{e 4}\right|^{2}\right) \sin ^{2} \phi},
$$

where $\phi$ is the difference of the phases of $U_{e 3}$ and $U_{e 4}$. Since $\phi$ is unknown, from Eq.(6.93) we obtain the constraints

$$
\left.|1-2| U_{e 4}\right|^{2}\left|m_{4} \lesssim\right|\langle m\rangle \mid \lesssim m_{4}
$$


where the upper and lower bounds correspond to $\phi=0$ or $\pi$ and $\phi= \pm \pi / 2$, respectively.29 Taking into account Eqs. (6.83) and (6.86), we have

$$
\left|U_{e 3}\right| \simeq \cos \vartheta_{\text {sun }} \quad \text { and } \quad\left|U_{e 4}\right| \simeq \sin \vartheta_{\text {sun }}
$$

and Eq.(6.94) can be written as

$$
m_{4} \sqrt{1-\sin ^{2} 2 \vartheta_{\text {sun }}} \lesssim|\langle m\rangle| \lesssim m_{4}
$$

If $\sin ^{2} 2 \vartheta_{\text {sun }} \ll 1$, which corresponds to the MSW solution with a small mixing angle, we have $|\langle m\rangle| \simeq m_{4}$, independent of the value of $\phi$ and of the conservation of CP. In the case of a large value of the parameter $\sin ^{2} 2 \vartheta_{\text {sun }}$, which correspond to the MSW solution with a large mixing angle or to the vacuum oscillation solution, in the future it will be possible to obtain information about the violation of $\mathrm{CP}$ in the lepton sector if both the ${ }^{3} \mathrm{H} \beta$-decay and neutrinoless double- $\beta$ decay experiments will obtain positive results. Indeed, from the measurements of $m\left({ }^{4} \mathrm{H}\right) \simeq m_{4},|\langle m\rangle|$ and $\sin ^{2} 2 \vartheta_{\text {sun }}$ with the help of Eq.(6.96) it will be possible to determine the value of $\sin ^{2} \phi$ (if $\sin ^{2} 2 \vartheta_{\text {sun }}$ is large). Let us emphasize that in the case of $\mathrm{CP}$ conservation the relative $\mathrm{CP}$ parities of $\nu_{3}$ and $\nu_{4}$ can be determined: the CP parities are equal if $\sin ^{2} \phi=0$ and opposite if $\sin ^{2} \phi=1$.

Considering now scheme B, the first inequality in Eq. (6.87) implies that both $m\left({ }^{3} \mathrm{H}\right)$ and $|\langle m\rangle|$ are strongly suppressed:

$$
\begin{aligned}
& m\left({ }^{3} \mathrm{H}\right) \simeq\left(\sum_{k=3,4}\left|U_{e k}\right|^{2}\right) m_{4} \leq a_{e}^{0} m_{4} \ll m_{4} . \\
& |\langle m\rangle| \simeq\left|\sum_{k=3,4} U_{e k}^{2}\right| m_{4} \leq\left(\sum_{k=3,4}\left|U_{e k}\right|^{2}\right) m_{4} \leq a_{e}^{0} m_{4} \ll m_{4} .
\end{aligned}
$$

Therefore, only if scheme $\mathrm{A}$ is realized in nature, there is some possibility to measure the effect of the LSND mass in future tritium decay experiments and neutrinoless double- $\beta$ decay experiments.

We discuss now the implications of the schemes A and B for LBL neutrino oscillation experiments 423. The smallness of $\sum_{k=1,2}\left|U_{e k}\right|^{2}$ in scheme A and of $\sum_{k=3,4}\left|U_{e k}\right|^{2}$ in scheme B implies that the electron neutrino has a small mixing with the neutrinos whose mass-squared difference is responsible for the oscillations of atmospheric neutrinos (i.e., $\nu_{1}, \nu_{2}$ in scheme A and $\nu_{3}, \nu_{4}$ in scheme B). Hence, as shown in the following part of this section, the transition probability of electron neutrinos and antineutrinos into other states in atmospheric and LBL experiments is suppressed.

Taking into account that in scheme $\mathrm{A}$ we have $\Delta m_{43}^{2} L / E \ll 1$ and $\Delta m_{41}^{2} L / E \gg 1$ in LBL experiments, we obtain from the general formula (3.12) the following expression for

\footnotetext{
${ }^{29}$ If CP is conserved, we have $\phi=0$ or $\pi$ if the CP parities of $\nu_{3}$ and $\nu_{4}$ are equal and $\phi= \pm \pi / 2$ if they are opposite. Indeed, if CP is conserved from Eq. (2.50) we get $U_{e k}^{*}=U_{e k} \rho_{k}$ where $\rho_{k}= \pm 1$ and the CP parity of $\nu_{k}$ is $\eta_{k}^{\mathrm{CP}}=i \rho_{k}$ (see Eq.(2.53) 96, 97]. Writing $U_{e k}=\left|U_{e k}\right| e^{i \phi_{k}}$, we obtain $\phi_{k}=0$ or $\pi$ if $\rho_{k}=+1$ and $\phi_{k}= \pm \pi / 2$ if $\rho_{k}=-1$. Hence, we have $\phi_{3}-\phi_{4}=0$ or $\pi$ if $\rho_{3}=\rho_{4}$ and $\phi_{3}-\phi_{4}= \pm \pi / 2$ if $\rho_{3}=-\rho_{4}$.
} 
the measurable probability of $\nu_{\alpha} \rightarrow \nu_{\beta}$ transitions averaged over the fast oscillations due to $\Delta m_{41}^{2}$ :

$$
P_{\nu_{\alpha} \rightarrow \nu_{\beta}}^{(\mathrm{LBL} ; \mathrm{A})}=\left|U_{\beta 1} U_{\alpha 1}^{*}+U_{\beta 2} U_{\alpha 2}^{*} \exp \left(-i \frac{\Delta m_{21}^{2} L}{2 E}\right)\right|^{2}+\left|\sum_{k=3,4} U_{\beta k} U_{\alpha k}^{*}\right|^{2} .
$$

The corresponding probability in scheme $\mathrm{B}$ is given by the exchange $1,2 \leftrightarrows 3,4$ and the probability of $\bar{\nu}_{\alpha} \rightarrow \bar{\nu}_{\beta}$ transitions is obtained changing $U_{\beta k} U_{\alpha k}^{*} \rightarrow U_{\beta k}^{*} U_{\alpha k}$.

Let us consider reactor LBL experiments which measure the survival probability $P_{\bar{\nu}_{e} \rightarrow \bar{\nu}_{e}}^{(\mathrm{LBL})}$. We will show that the negative results of SBL reactor experiments and the results of solar neutrino experiments imply strong constraints on the transition probability of LBL reactor $\bar{\nu}_{e}$ 's into other states. Indeed, from Eq. (6.99) and the corresponding one in scheme B, for the survival probability of LBL reactor $\bar{\nu}_{e}$ 's we have the lower bounds

$$
\begin{aligned}
& P_{\bar{\nu}_{e} \rightarrow \bar{\nu}_{e}}^{(\mathrm{LBL} ; \mathrm{A})} \geq\left(\sum_{k=3,4}\left|U_{e k}\right|^{2}\right)^{2}=\left(1-\sum_{k=1,2}\left|U_{e k}\right|^{2}\right)^{2}, \\
& P_{\bar{\nu}_{e} \rightarrow \bar{\nu}_{e}}^{(\mathrm{LBL} ; \mathrm{B})} \geq\left(\sum_{k=1,2}\left|U_{e k}\right|^{2}\right)^{2}=\left(1-\sum_{k=3,4}\left|U_{e k}\right|^{2}\right)^{2},
\end{aligned}
$$

in schemes A and B, respectively. From the inequalities (6.86) and (6.87) it follows that in both schemes

$$
P_{\bar{\nu}_{e} \rightarrow \bar{\nu}_{e}}^{(\mathrm{LBL})} \geq\left(1-a_{e}^{0}\right)^{2} .
$$

Since $a_{e}^{0}$ is small (see Fig. 6.2 and Eq.(6.66)), the lower bound (6.102) implies that the survival probability $P_{\bar{\nu}_{e} \rightarrow \bar{\nu}_{e}}^{(\mathrm{LBL})}$ is close to one. For the transition probability of $\nu_{e}$ 's into any other state in LBL experiments,

$$
1-P_{\bar{\nu}_{e} \rightarrow \bar{\nu}_{e}}^{(\mathrm{LBL})}=\sum_{\beta \neq e} P_{\bar{\nu}_{e} \rightarrow \bar{\nu}_{\beta}}^{(\mathrm{LBL})}
$$

we have the upper bound

$$
1-P_{\bar{\nu}_{e} \rightarrow \bar{\nu}_{e}}^{(\mathrm{LBL})} \leq a_{e}^{0}\left(2-a_{e}^{0}\right) .
$$

The value of this bound, which depends on $\Delta m_{41}^{2}$ through the dependence of $a_{e}^{0}$ on $\Delta m_{41}^{2}$ (see Fig. 6.2), is shown in Fig 6.4 (the solid line), where it is compared with the upper bound for $1-P_{\bar{\nu}_{e} \rightarrow \bar{\nu}_{e}}^{(\mathrm{LBL})}$ obtained in the CHOOZ experiment 159 (dash-dotted line) and with the final sensitivity of the CHOOZ experiment (dash-dot-dotted line). The shadowed region in Fig 6.4 corresponds to the range of $\Delta m_{41}^{2}$ allowed at $90 \%$ CL by the results of the LSND and other experiments. One can see from Fig 6.4 that if $\Delta m_{41}^{2}$ lies in the LSND range, the upper bound for $1-P_{\bar{\nu}_{e} \rightarrow \bar{\nu}_{e}}^{(\mathrm{LBL})}$ is much less than the sensitivity of the CHOOZ experiment and it will be very difficult to measure the disappearance of reactor $\bar{\nu}_{e}$ in future LBL experiments.

Let us consider now $\nu_{\mu} \rightarrow \nu_{e}$ transitions, which will be investigated in the near future by the K2K 271, MINOS [293, ICARUS [294] and/or other 295 298 experiments 


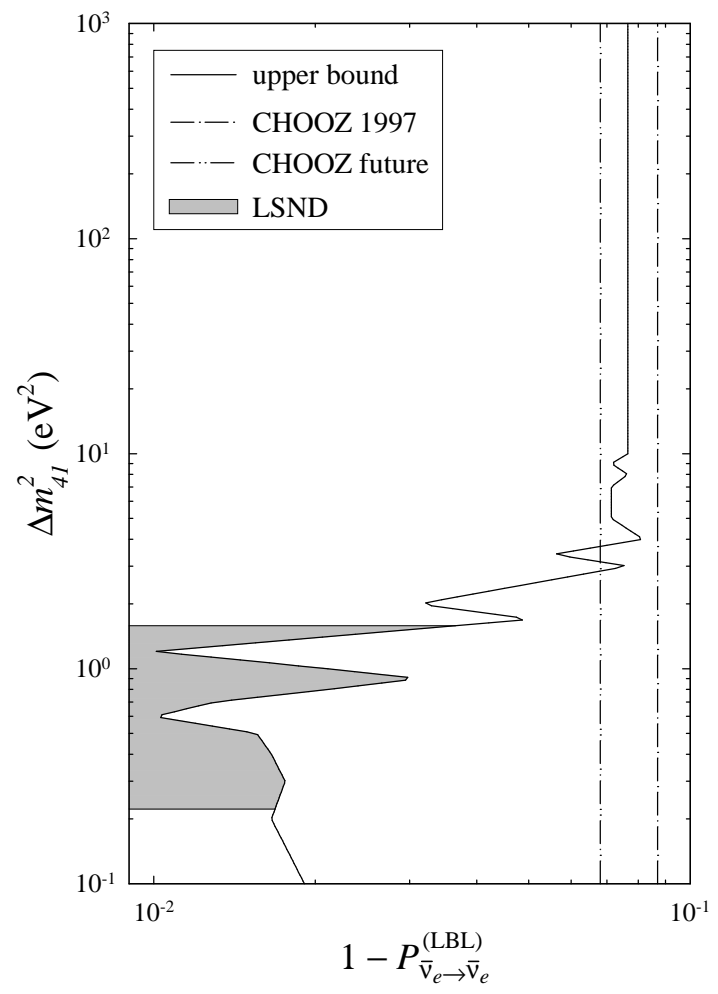

Figure 6.4. The value of the bound (6.104) on the transition probability of $\bar{\nu}_{e}$ into all other neutrino types in LBL experiments as a function of $\Delta m_{41}^{2}$ (solid line). The dash-dotted and dashdot-dotted lines represent the upper bound obtained in the CHOOZ experiment [159] and the final sensitivity planned in this experiment, respectively. The shadowed region corresponds to the range of $\Delta m_{41}^{2}$ allowed at $90 \%$ CL by the results of LSND and other experiments.

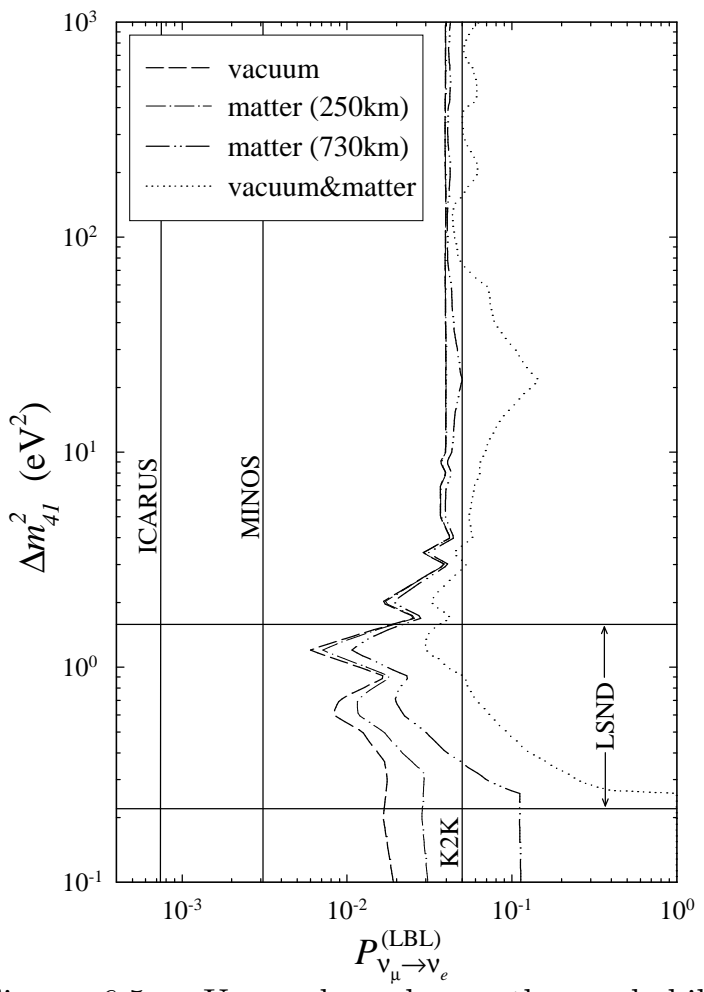

Figure 6.5. Upper bounds on the probability of $\nu_{\mu} \rightarrow \nu_{e}$ transitions in LBL experiments as functions of $\Delta m_{41}^{2}$. The dashed line represents the bound (6.109), whereas the dash-dotted and dash-dot-dotted curves include matter corrections to this bound for the $\mathrm{K} 2 \mathrm{~K}(L=250 \mathrm{~km})$ and MINOS and ICARUS ( $L=730 \mathrm{~km})$ experiments, respectively. The vertical lines show the planned sensitivities of these experiments. The dotted curve depicts the bound (6.111). The range of $\Delta m_{41}^{2}$ allowed by LSND and other experiments is also indicated.

(see [414]). With the help of the Cauchy-Schwarz inequality, from Eq. 6.99) and Eq.(6.60) we obtain

$$
P_{\nu_{\mu} \rightarrow \nu_{e}}^{(\mathrm{LBL})} \leq\left(\sum_{k}\left|U_{e k}\right|^{2}\right)\left(\sum_{k}\left|U_{\mu k}\right|^{2}\right)+\frac{1}{4} A_{\mu ; e}
$$

where the index $k$ runs over 1,2 in the scheme A and over 3,4 in the scheme B. From the constraints (6.86) and (6.87), in both schemes $\mathrm{A}$ and $\mathrm{B}$ we have

$$
P_{\nu_{\mu} \rightarrow \nu_{e}}^{(\mathrm{LBL})} \leq a_{e}^{0}+\frac{1}{4} A_{\mu ; e}^{0}
$$

where $A_{\mu ; e}^{0}$ is the upper bound for the oscillation amplitude $A_{\mu ; e}$ measured in SBL experiments.

Since the CPT relation (3.15) and the conservation of probability, relation (6.103), 
imply that

$$
\sum_{\alpha \neq e} P_{\nu_{\alpha} \rightarrow \nu_{e}}^{(\mathrm{LBL})}=\sum_{\alpha \neq e} P_{\bar{\nu}_{e} \rightarrow \bar{\nu}_{\alpha}}^{(\mathrm{LBL})}=1-P_{\bar{\nu}_{e} \rightarrow \bar{\nu}_{e}}^{(\mathrm{LBL})}
$$

another upper limit for $P_{\nu_{\mu} \rightarrow \nu_{e}}^{(\mathrm{LBL})}$ can be obtained from the inequality (6.104):

$$
P_{\nu_{\mu} \rightarrow \nu_{e}}^{(\mathrm{LBL})} \leq a_{e}^{0}\left(2-a_{e}^{0}\right) .
$$

Combining the upper bounds (6.106) and (6.108) and taking into account that they are valid also for antineutrinos [423], we have

$$
\underset{\substack{(-) \\ \nu_{\mu} \rightarrow \nu_{e}}}{(\mathrm{LBL})} \leq \operatorname{Min}\left(a_{e}^{0}+\frac{1}{4} A_{\mu ; e}^{0}, a_{e}^{0}\left(2-a_{e}^{0}\right)\right) .
$$

The curve corresponding to this limit obtained from the $90 \%$ CL exclusion plots of the Bugey [162] experiment for $a_{e}^{0}$ and of the BNL E734 [372], BNL E776 [373], CCFR [374] and KARMEN (Bayesian analysis) [375] experiments for $A_{\mu ; e}^{0}$ is represented by the dashed line in Fig. 6.5. For comparison, the expected sensitivities of the LBL accelerator neutrino experiments K2K [271], MINOS [293] and ICARUS [294] are also indicated (the three straight vertical lines). The dash-dotted and dash-dot-dotted lines in Fig. 6.5 represent, respectively, the upper bound for $P_{\nu_{\mu} \rightarrow \nu_{e}}^{(\mathrm{LBL})}$ in the K2K and MINOS, ICARUS experiments corrected for the matter effects [123] due to the propagation of the neutrino beam in the crust of the earth. These effects are different for the K2K and MINOS, ICARUS experiments because of the different value of $L$ in this experiments: $L \simeq 250 \mathrm{~km}$ in K2K and $L \simeq 730 \mathrm{~km}$ in MINOS and ICARUS. (It is obvious that the bound for other LBL experiments with a neutrino beam from CERN to Gran Sasso is the same as that for the ICARUS experiment.)

The region between the two horizontal solid lines in Fig. 6.5 corresponds to the range of $\Delta m_{41}^{2}$ allowed at $90 \%$ CL by the results of the LSND and other experiments. One can see that if $\Delta m_{41}^{2}$ is in the LSND region, the sensitivity of the K2K experiment is not sufficient for the observation of $\nu_{\mu} \rightarrow \nu_{e}$ transitions, whereas experiments with higher sensitivities like MINOS and ICARUS could reveal these transitions.

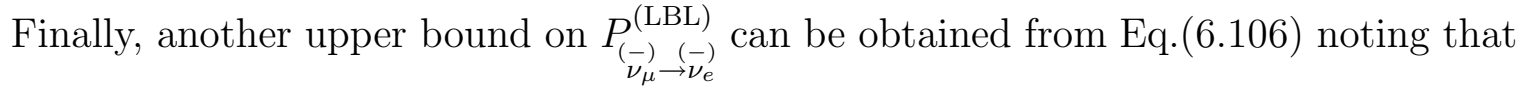

$$
A_{\mu ; e} \leq 4\left(1-\sum_{k}\left|U_{\mu k}\right|^{2}\right)\left(1-\sum_{k}\left|U_{e k}\right|^{2}\right),
$$

with $k=1,2$ in scheme $\mathrm{A}$ and $k=3,4$ in scheme $\mathrm{B}$. Taking into account the inequalities (6.86) and (6.87), we obtain

$$
P_{(-),(-)}^{(\mathrm{LBL})} \leq a_{e}^{0}+a_{\mu}^{0}-2 a_{e}^{0} a_{\mu}^{0}
$$

This bound is stable against matter effects [423] and its value is represented by the dotted curve in Fig. 6.5. For $a_{\mu}^{0} \ll a_{e}^{0} \ll 1$ this bound is about half of that given by Eq. (6.108). 


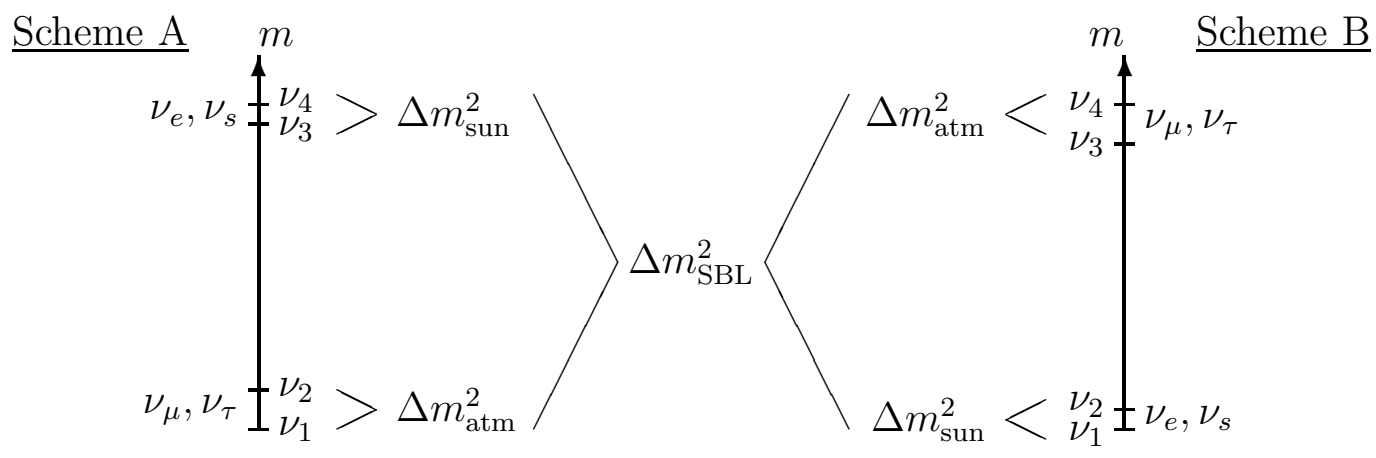

Figure 6.6. The two types of neutrino mass spectra that can accommodate the solar, atmospheric and LSND scales of $\Delta m^{2}$ and the mixing schemes that emerge if the Big-Bang Nucleosynthesis constraint on the number of light neutrinos is less than 4 .

However, since $a_{\mu}^{0}$ is only small in the same range of $\Delta m^{2}$ where $A_{\mu ; e}^{0}$ is small, numerically the bound (6.111) turns out to be worse than the bound (6.109) (dashed line in Fig. 6.5) and the corresponding matter-corrected bound (dash-dotted and dash-dot-dotted lines in Fig. 6.5).

Summarizing the results presented in this section, we have shown that only the two four-neutrino schemes $\mathrm{A}$ and $\mathrm{B}$ are compatible with the results of all neutrino oscillation experiments. If scheme $\mathrm{A}$ is realized in nature, the effect of non-zero neutrino masses could be observed in the near future in tritium $\beta$-decay experiments and neutrinoless double- $\beta$ decay experiments, whereas these effects are strongly suppressed in scheme B. Furthermore, we have shown that the results of SBL $\bar{\nu}_{e}$ disappearance experiments and solar neutrino experiments imply that in both schemes A and B the electron neutrino has a small mixing with the neutrinos whose mass-squared difference is responsible for the oscillations of atmospheric neutrinos (i.e., $\nu_{1}, \nu_{2}$ in scheme A and $\nu_{3}, \nu_{4}$ in scheme B). As a consequence, the transition probability of electron neutrinos and antineutrinos into other states in atmospheric and LBL experiments is strongly suppressed.

Furthermore, it has been shown in Refs. 417, 424 that in the schemes A and B, if the standard Big-Bang Nucleosynthesis constraint on the number of light neutrinos (see 425] and references therein) is less than 4 , then one has a stringent limit on the mixing of the sterile neutrino with the two massive neutrinos that are responsible for the oscillations of atmospheric neutrinos. In this case, $\nu_{s}$ is mainly mixed with the two massive neutrinos that contribute to solar neutrino oscillations $\left(\nu_{3}\right.$ and $\nu_{4}$ in scheme $\mathrm{A}$ and $\nu_{1}$ and $\nu_{2}$ in scheme B) and the unitarity of the mixing matrix implies that $\nu_{\tau}$ is mainly mixed with the two massive neutrinos that contribute to the oscillations of atmospheric neutrinos. Hence, the two schemes have the form shown in Fig. 6.6 and have the following testable implications for solar, atmospheric, long-baseline and short-baseline neutrino oscillation experiments:

- The solar neutrino problem is due to $\nu_{e} \rightarrow \nu_{s}$ oscillations. This prediction will be checked by future solar neutrino experiments that can measure the ratio of neutralcurrent and charged-current events [356]. 
- The atmospheric neutrino anomaly is due to $\nu_{\mu} \rightarrow \nu_{\tau}$ oscillations. This prediction will be investigated by LBL experiments. Furthermore, the absence of $\nu_{\mu} \rightarrow \nu_{s}$ atmospheric neutrino oscillations may be checked in the future in the Super-Kamiokande atmospheric neutrino experiment [271, 272, 275, 276].

- $\nu_{\mu} \rightarrow \nu_{\tau}$ and $\nu_{e} \rightarrow \nu_{s}$ oscillations are strongly suppressed in SBL experiments [424].

If these prediction will be falsified by future experiments it could mean that some of the indications given by the results of neutrino oscillations experiments are wrong and neither of the two four neutrino schemes A and B is realized in nature, or that Big-Bang Nucleosynthesis occurs with a non-standard mechanism [426]. On the other hand, if the standard Big-Bang Nucleosynthesis constraint on the number of light neutrinos is less than 4 and one of the two four neutrino schemes depicted in Fig. 6.6 is realized in nature, at the leading order the $4 \times 4$ neutrino mixing matrix has an extremely simple structure in which the $\nu_{e}, \nu_{s}$ and $\nu_{\mu}, \nu_{\tau}$ sectors are decoupled: in scheme A

$$
U \simeq\left(\begin{array}{cccc}
0 & 0 & \cos \vartheta_{\text {sun }} & \sin \vartheta_{\text {sun }} \\
\cos \vartheta_{\text {atm }} & \sin \vartheta_{\text {atm }} & 0 & 0 \\
-\sin \vartheta_{\text {atm }} & \cos \vartheta_{\text {atm }} & 0 & 0 \\
0 & 0 & -\sin \vartheta_{\text {sun }} & \cos \vartheta_{\text {sun }}
\end{array}\right)
$$

and in scheme B

$$
U \simeq\left(\begin{array}{cccc}
\cos \vartheta_{\text {sun }} & \sin \vartheta_{\text {sun }} & 0 & 0 \\
0 & 0 & \cos \vartheta_{\text {atm }} & \sin \vartheta_{\text {atm }} \\
0 & 0 & -\sin \vartheta_{\text {atm }} & \cos \vartheta_{\text {atm }} \\
-\sin \vartheta_{\text {sun }} & \cos \vartheta_{\text {sun }} & 0 & 0
\end{array}\right)
$$

where $\vartheta_{\text {sun }}$ and $\vartheta_{\text {atm }}$ are, respectively, the two-generation mixing angles relevant in solar and atmospheric neutrino oscillations. Therefore, the oscillations of solar and atmospheric neutrinos are independent and the two-generation analyses of solar and atmospheric neutrino oscillations yield correct information on the mixing of four neutrinos. The $\stackrel{(-)}{\nu_{\mu}} \rightarrow \stackrel{(-)}{\nu}$ transitions observed in the LSND experiment represent only a slight distortion of this simple picture.

\section{Conclusions}

In this review, we have discussed the phenomenological aspects of neutrino oscillations. The phenomenon of neutrino oscillations is possible if neutrinos are massive and mixed particles. The problem of neutrino masses and mixing is the central problem of today's neutrino physics and astrophysics. More than 40 different experiments all over the world are dedicated to the investigation of this problem and several new experiments are in preparation.

The investigation of the properties of neutrinos is considered as one of the most important direction for the search of new physics (see Ref. [31]). Massive neutrinos are 
among the plausible candidates for dark matter particles and the number of massive light neutrinos plays crucial role in Big-Bang Nucleosynthesis 425.

The Super-Kamiokande collaboration obtained recently a rather convincing evidence in favour of transitions of atmospheric $\nu_{\mu}$ into other neutrino states $\left(\nu_{\tau}\right.$ or $\left.\nu_{\text {sterile }}\right)$. Other indications in favour of neutrino mixing were obtained in all solar neutrino experiments and in the accelerator LSND experiment.

If all these indications will be confirmed by future experiments, it will mean that the number of massive light neutrinos is larger than the number of flavour neutrinos, i.e. that sterile neutrinos exist. This would be an evidence that neutrino masses and mixing are phenomena due to physics beyond the Standard Model with right-handed neutrino singlets.

If the indications in favour of a "large" $\Delta m^{2} \sim 1 \mathrm{eV}^{2}$ obtained in the LSND experiment will not be confirmed by future experiments, the mixing of three massive neutrinos with a mass hierarchy is a possible and plausible scenario. In this case, the Dirac or Majorana nature of massive neutrinos can be determined only increasing the sensitivity of the experiments on the search for neutrinoless double- $\beta$ decay by almost two orders of magnitude. It has been shown by an analysis of the existing data [427, 428] that the effective Majorana mass in the case of three massive Majorana neutrinos and a mass hierarchy is smaller than $10^{-2} \mathrm{eV}$, whereas the present upper bound is about $0.5 \mathrm{eV}$ [429, 430] and the sensitivity of the next generation of experiments will be of the order of $10^{-1} \mathrm{eV}$ 431].

Future neutrino oscillation experiments will make possible to investigate in detail the regions of $\Delta m^{2}$ and of the elements of the neutrino mixing matrix allowed by the existing results of neutrino oscillation experiments. Future solar neutrino experiments (SNO, ICARUS, Borexino, GNO and others [353]) will provide model-independent information on the transition of high energy ${ }^{8} \mathrm{~B}$ neutrinos into other states and investigate in detail the fluxes of medium energy ${ }^{7} \mathrm{Be}$, pep and $\mathrm{CNO}$ neutrinos. Future accelerator LBL experiments (K2K 271, MINOS 293], ICARUS 294 and others 295 298, 414]) will explore the region of $\Delta m^{2}$ indicated by the atmospheric neutrino anomaly. Besides $\nu_{\mu} \rightarrow \nu_{\mu}$ survival, also $\nu_{\mu} \rightarrow \nu_{\tau}$ and $\nu_{\mu} \rightarrow \nu_{e}$ transitions will be studied. A very important task for the neutrino oscillation experiments of the next generation is the check of the indication in favour of neutrino oscillations obtained in the LSND experiment. The KARMEN experiment will reach the LSND sensitivity in one or two years [379]. The proposed $\nu_{\mu} \rightarrow \nu_{e}$ SBL experiments BooNE [387 at Fermilab, I-216 388] at CERN, ORLaND [389] at Oak Ridge and NESS at the European Spallation Source [390] plan to achieve a sensitivity about two orders of magnitude better than the one of the LSND experiment.

It is now widely believed that the recent measurement of an up-down asymmetry of atmospheric $\mu$-like events in the Super-Kamiokande detector represents a strong evidence in favour of neutrino oscillations generated by new physics beyond the Standard Model (see, for example, Ref. 432]). We think that in order to understand the origin of neutrino masses and mixing it is necessary to investigate in much more detail all the phenomena generated by these properties: neutrino oscillations, neutrinoless double- $\beta$ decay, distortion of the high-energy part of the electron spectrum of tritium $\beta$ decay, 
neutrino magnetic moments, etc. There is no doubt that the next generation of neutrino experiments will lead to a great progress in the understanding the properties of neutrinos, the most puzzling among the known particles.

\section{Note added}

After this review was finished and presented to the editor (December 15, 1998), new Super-Kamiokande data were reported at WIN99 (Capetown, January 24 - 30, 1999) by Y. Suzuki and at the VIIIth International Workshop on Neutrino Telescopes (Venice, February 23 - 26, 1999) by K. Scholberg and K. Inoue. Also Soudan-2 published an updated result. Below we will shortly present the new development.

\section{Atmospheric neutrinos}

For the double ratio $R(5.8)$ the new values based on 736 days for FC events and 685 days for PC events are

$$
R= \begin{cases}0.67 \pm 0.02 \pm 0.05 & \text { sub-GeV } \\ 0.66 \pm 0.04 \pm 0.08 & \text { multi-GeV }\end{cases}
$$

For the up-down asymmetry (5.10) of $\mu$-like events in the multi-GeV region the new value

$$
A_{\mu}=-0.311 \pm 0.043 \pm 0.01
$$

is more than 7 standard deviations away from zero. Thus, the new data confirm the SuperKamiokande evidence in favour of oscillations of atmospheric neutrinos. The updated dependence of the up-down asymmetry $A$ as a function of the lepton momentum for $e$-like and $\mu$-like events is given in Fig. A. The new allowed region in the plane of the oscillation parameters under the assumption of $\nu_{\mu} \rightarrow \nu_{\tau}$ oscillations is shown in Fig. B. The region allowed by Kamiokande and the allowed region found from the analysis of previous Super-Kamiokande data (see Fig. 5.1) are also depicted. As it is seen from Fig. B, the new Super-Kamiokande data favour higher values of $\Delta m^{2}$ than the previous ones. The best-fit values of the parameters are

$$
\sin ^{2} 2 \vartheta=1, \quad \Delta m^{2}=3.5 \times 10^{-3} \mathrm{eV}^{2} .
$$

This fit corresponds to $\chi_{\min }^{2}=62.1$ for 67 DOF (the previous best-fit value of $\Delta m^{2}$ was $\left.2.2 \times 10^{-3} \mathrm{eV}^{2}\right)$. The new allowed range for $\Delta m^{2}$ at $90 \%$ CL is $10^{-3} \mathrm{eV}^{2} \lesssim \Delta m^{2} \lesssim$ $8 \times 10^{-3} \mathrm{eV}^{2}$.

The Super-Kamiokande Collaboration also measured the zenith angle dependence of the number of up-going muon events (for a discussion see Section 5.1.2). Also these data are compatible with the hypothesis of $\nu_{\mu} \rightarrow \nu_{\tau}$ oscillations. The best-fit values of the oscillation parameters obtained from the data on the angular dependence of throughgoing muon events are $\sin ^{2} 2 \vartheta=1$ and $\Delta m^{2}=3.2 \times 10^{-3} \mathrm{eV}^{2}$ (corresponding to $\chi_{\min }^{2}=7$ for $8 \mathrm{DOF})$. 


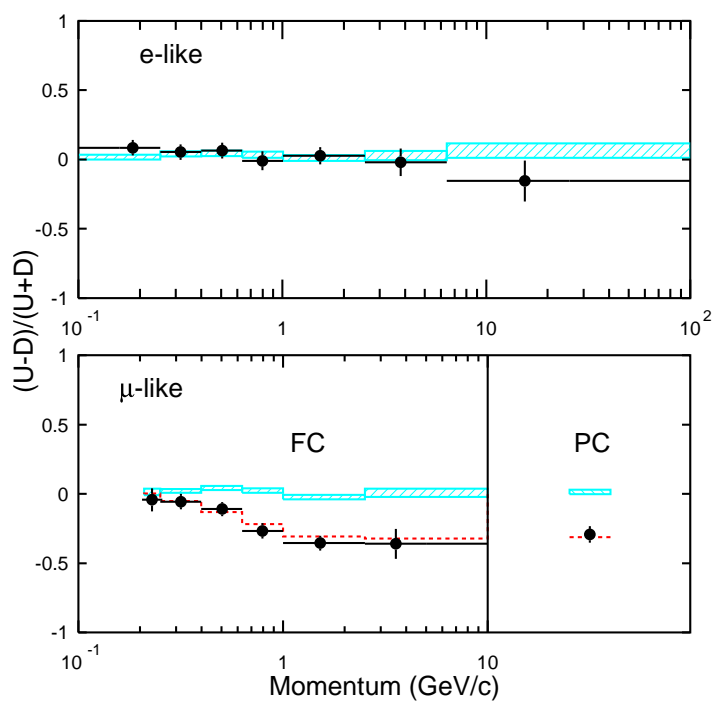

Figure A. The up-down asymmetry $A$ of $e$-like and $\mu$-like events as a function of the lepton momentum. The hatched region shows the theoretical expectation without neutrino oscillations. The dashed line for $\mu$-like events represents the fit of the data in the case of two-generation $\nu_{\mu} \rightarrow \nu_{\tau}$ oscillations with $\Delta m^{2}=3.5 \times 10^{-3} \mathrm{eV}^{2}$ and $\sin ^{2} 2 \vartheta=1.0$.

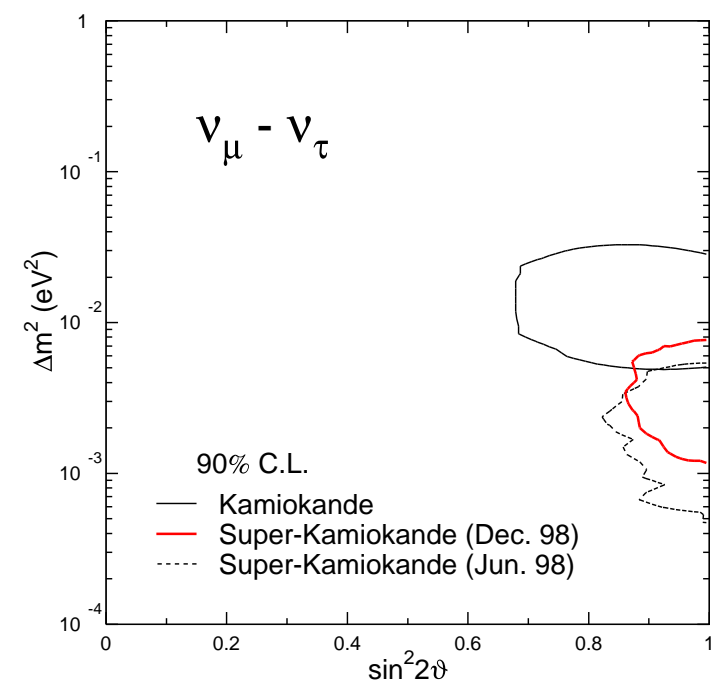

Figure B. The region allowed at 90\% CL in the $\sin ^{2} 2 \vartheta-\Delta m^{2}$ plane for $\nu_{\mu} \rightarrow \nu_{\tau}$ oscillations obtained by Super-Kamiokande (December 98). For comparison, the allowed regions found by the Kamiokande Collaboration and by the SuperKamiokande Collaboration (June 98) are also shown.

The atmospheric neutrino FC and PC data can also be described if one assumes that $\nu_{\mu} \rightarrow \nu_{s}$ transitions take place. In this case the best-fit values of the oscillation parameters are given by $\sin ^{2} 2 \vartheta=1$ and $\Delta m^{2}=4.5 \times 10^{-3} \mathrm{eV}^{2}$ with $\chi_{\min }^{2}=64.3$ for 67 DOF.

The Super-Kamiokande Collaboration reported also the observation of $231.8 \mathrm{NC} \pi^{0}$ events (see Eq.(5.11)), after subtraction of background, with two $e$-like tracks with an invariant mass in the range from 90 to $180 \mathrm{MeV}$. As a preliminary result it was found that

$$
\left(\frac{\pi^{0}}{e}\right)_{\mathrm{data}} /\left(\frac{\pi^{0}}{e}\right)_{\mathrm{MC}}=1.11 \pm 0.06 \text { (data stat) } \pm 0.02 \text { (MC stat) } \pm 0.26 \text { (syst) }
$$

In the case of $\nu_{\mu} \rightarrow \nu_{\tau}$ transitions this ratio should be equal to one, whereas in the case of $\nu_{\mu} \rightarrow \nu_{s}$ transitions this ratio should be about 0.75 . The large systematic error in the present data does not allow to reach a definite conclusion on the transition channel.

The updated value for the double ratio $R$ of the Soudan-2 experiment is $R=0.64 \pm$ $0.11 \pm 0.06$ (W.W.M. Allison et al., hep-ex/9901024).

\section{Solar neutrinos}

In the region of recoil electron energies between 6.5 and $20 \mathrm{MeV}$, during 708 days, the Super-Kamiokande Collaboration has observed $9531_{-155}^{+167}$ events (about 13.5 events per day). For the value of the ${ }^{8} \mathrm{~B}$ neutrino flux it was found $\Phi_{{ }_{8}}^{\mathrm{SK}}=(2.42 \pm 0.04 \pm 0.07) \times 10^{6}$ 
$\mathrm{cm}^{-2} \mathrm{~s}^{-1}$. The ratio of this flux to the flux predicted by the SSM 304 is equal to $0.470 \pm$ $0.008 \pm 0.013$.

Now Super-Kamiokande has also data with an energy of the recoil electrons in the range from 5.5 to $6.5 \mathrm{MeV}$. This lowering of the electron energy threshold is the most important difference of the new data with respect to the previous ones. The best fit to the recoil energy spectrum is given by vacuum oscillations with the best-fit parameters $\sin ^{2} 2 \vartheta=0.80$ and $\Delta m^{2}=4.3 \times 10^{-10} \mathrm{eV}^{2}$. This is mainly because of the observed excess of events with respect to the SSM prediction in the high energy region of the recoil electron spectrum.

The contribution to the recoil spectrum in the region $E_{e} \gtrsim 14 \mathrm{MeV}$ comes from hep neutrinos, which according to the SSM is small (see Table 5.2). If the flux of hep neutrinos is considered as a free parameter, then from a fit of the Super-Kamiokande data under the assumption of absence of neutrino oscillations it was found $\Phi_{h e p}^{\mathrm{fit}}=\left(14.0_{-6.4}^{+7.6}\right) \Phi_{h e p}^{\mathrm{SSM}}$. The contribution of hep neutrinos in the Super-Kamiokande recoil energy spectrum was analysed in detail in the papers of J. Bahcall and P. Krastev, Phys. Lett. B 436, 243 (1998) and G. Fiorentini et al., Phys. Lett. B 444, 387 (1998).

Super-Kamiokande also presented a new measurement of the day-night asymmetry with the result $N / D-1=0.060 \pm 0.036_{-0.027}^{+0.028}$.

\section{Acknowledgements}

S.M.B. acknowledges the support of the "Sonderforschungsbereich 375-95 für AstroTeilchenphysik der Deutschen Forschungsgemeinschaft". C.G. would like to express his gratitude to the Korea Institute for Advanced Study (KIAS) for the kind hospitality during part of this work. Furthermore, we thank V.A. Naumov for reading a preliminary version of the manuscript and providing several useful comments. We are particularly grateful to K. Scholberg for providing us with the latest Super-Kamiokande figures.

\section{A Properties of Majorana neutrinos and fields}

In this Appendix we summarize the main properties of Majorana particles associated with Majorana fields. These particles have spin $1 / 2$ and all charges equal to zero. A free Majorana field $\nu(x)$ satisfies the Dirac equation

$$
\left(i \gamma^{\mu} \partial_{\mu}-m\right) \nu(x)=0
$$

together with the Majorana condition

$$
\nu(x)=\nu^{c}(x) \equiv \mathcal{C} \bar{\nu}^{T}(x)
$$

where $\nu^{c}$ is the charge-conjugate field and $\mathcal{C}$ is the charge-conjugation matrix which fulfills the relations

$$
\mathcal{C} \gamma_{\mu}^{T} \mathcal{C}^{-1}=-\gamma_{\mu}, \quad \mathcal{C}^{\dagger}=\mathcal{C}^{-1}, \quad \mathcal{C}^{T}=-\mathcal{C}
$$


From Eqs.A.1) and (A.2) it follows that the Fourier expansion of the Majorana field is

$$
\nu(x)=\int \frac{\mathrm{d}^{3} p}{\sqrt{(2 \pi)^{3} 2 p_{0}}} \sum_{r= \pm 1}\left[a_{r}(p) u^{r}(p) e^{-i p x}+a_{r}^{\dagger}(p) v^{r}(p) e^{i p x}\right],
$$

where the spinors $u^{r}(p)$ and $v^{r}(p)$ are related by

$$
v^{r}(p)=\mathcal{C}\left(\bar{u}^{r}(p)\right)^{T}
$$

The operators $a_{r}(p)$ and $a_{r}^{\dagger}(p)$ annihilate and create Majorana particles with momentum $p$ and helicity $r$ (particle $\equiv$ antiparticle for a Majorana field), respectively.

Let us consider the quantities

$$
\overline{\nu_{a}} \mathcal{O}_{j} \nu_{b}
$$

where $\nu_{a}$ and $\nu_{b}$ are two Majorana fields and

$$
\mathcal{O}_{j}=1, \gamma_{\mu}, \sigma_{\mu \nu}, \gamma_{\mu} \gamma_{5}, \gamma_{5}, \quad \text { for } \quad j=S, V, T, A, P \text {. }
$$

Taking into account the minus sign that appears under interchange of two fermion operators and the Majorana condition (A.2), we obtain

$$
\overline{\nu_{a}} \mathcal{O}_{j} \nu_{b}=\overline{\nu_{b}} \mathcal{C} \mathcal{O}_{j}^{T} \mathcal{C}^{-1} \nu_{a}
$$

From Eq.(A.3) we derive

$$
\mathcal{C} \sigma_{\mu \nu}^{T} \mathcal{C}^{-1}=-\sigma_{\mu \nu} \quad \text { and } \quad \mathcal{C} \gamma_{5}^{T} \mathcal{C}^{-1}=\gamma_{5}
$$

With the help of this equation and Eq.(A.3) it follows that

$$
\overline{\nu_{a}} \mathcal{O}_{j} \nu_{b}=\eta_{j} \overline{\nu_{b}} \mathcal{O}_{j} \nu_{a}
$$

with

$$
\eta_{j}=-1 \quad \text { for } \quad j=V, T \quad \text { and } \quad \eta_{j}=1 \quad \text { for } \quad j=S, A, P .
$$

Thus, for a single Majorana field $\nu=\nu_{a}=\nu_{b}$ we obtain

$$
\bar{\nu} \gamma_{\mu} \nu=0 \quad \forall \mu \quad \text { and } \quad \bar{\nu} \sigma_{\lambda \rho} \nu=\bar{\nu} \sigma_{\lambda \rho} \gamma_{5} \nu=0 \quad \forall \lambda, \rho
$$

This means that a Majorana particle has neither electric charge nor magnetic moments or electric dipole moments.

For any fermion field there is a relation between its left-handed component and the right-handed component of its charge-conjugate field (see Section 2.2):

$$
\psi_{R}=\left(\left(\psi^{c}\right)_{L}\right)^{c}
$$

In the case of a Dirac field, $\psi^{c}$ is different from $\psi$ and, therefore, the left and right-handed components are independent, whereas from Eq.(A.13) it follows for a Majorana field $\nu$ that

$$
\nu_{R}=\left(\nu_{L}\right)^{c}
$$


and thus the left and right-handed components of a Majorana field are not independent (in other words, a Majorana field is a two-component field).

Though Dirac and Majorana neutrinos differ in the number of degrees of freedom, it is nevertheless difficult to distinguish between the two in practice. The reason is that in the standard weak interaction Lagrangians (2.1) and (2.2) only the left-handed components $\nu_{\ell L}(\ell=e, \mu, \tau)$ of the flavour neutrino fields are present. These are connected with the left-handed components $\nu_{k L}$ of the neutrino mass eigenfields by the relation $\nu_{\ell L}=\sum_{k} U_{\ell k} \nu_{k L}$ (see Section 2). The mass eigenfields could be of Dirac or Majorana type. In the first case we have $\nu_{k}^{D}=\nu_{k L}+\nu_{k R}$, where the $\nu_{k R}$ are independent degrees of freedom, whereas in the second case we have $\nu_{k}^{M}=\nu_{k L}+\left(\nu_{k L}\right)^{c}$. The only difference between the Dirac and Majorana case with respect to the standard weak interactions resides in the mass term. In the limit of vanishing neutrino masses the right-handed Dirac fields decouple from the standard weak interaction Lagrangians and the distinction between Dirac and Majorana gets lost. Then the physical fields are simply given by the $\nu_{\ell L}$. In summary, we have arrived at the following conclusion 433: With the standard weak interactions one cannot distinguish between the Dirac and Majorana nature of the neutrinos in the limit of vanishing neutrino masses. In other words, for the standard weak interactions, Dirac neutrinos with negative helicity and Dirac antineutrinos with positive helicity are the same as Majorana neutrinos with positive and negative helicities, respectively, in this limit.

In neutrinoless double- $\beta$ decay, which can occur only with Majorana neutrinos (see Section 6), one needs to calculate the propagator

$$
\left\langle 0\left|T\left(\nu_{e L}\left(x_{1}\right) \nu_{e L}^{T}\left(x_{2}\right)\right)\right| 0\right\rangle .
$$

With the Majorana mass eigenfields $\nu_{k}$ satisfying the relation $\nu_{k}^{T}=-\bar{\nu}_{k} \mathcal{C}$ this propagator is obtained by the following steps:

$$
\begin{aligned}
\langle 0| T & \left(\nu_{e L}\left(x_{1}\right) \nu_{e L}^{T}\left(x_{2}\right)\right)|0\rangle \\
& =\frac{1-\gamma_{5}}{2} \sum_{k} U_{e k}^{2}\left\langle 0\left|T\left(\nu_{k}\left(x_{1}\right) \nu_{k}^{T}\left(x_{2}\right)\right)\right| 0\right\rangle \frac{1-\gamma_{5}^{T}}{2} \\
& =-\frac{1-\gamma_{5}}{2} \sum_{k} U_{e k}^{2}\left\langle 0\left|T\left(\nu_{k}\left(x_{1}\right) \bar{\nu}_{k}\left(x_{2}\right)\right)\right| 0\right\rangle \frac{1-\gamma_{5}}{2} \mathcal{C} \\
& =-\sum_{k} U_{e k}^{2} m_{k} i \int \frac{d^{4} p}{(2 \pi)^{4}} \frac{e^{-i p\left(x_{1}-x_{2}\right)}}{p^{2}-m_{k}^{2}} \frac{1-\gamma_{5}}{2} \mathcal{C} .
\end{aligned}
$$

Finally, we want to prove that the $\mathrm{CP}$ parity of a Majorana field is $\pm i$. The $\mathrm{CP}$ transformation of a Majorana field $\nu(x)$ is given by

$$
U_{\mathrm{CP}} \nu(x) U_{\mathrm{CP}}^{-1}=\eta_{\mathrm{CP}} \gamma_{0} \nu\left(x_{\mathrm{P}}\right),
$$

where the phase factor $\eta_{\mathrm{CP}}$ is the $\mathrm{CP}$ parity of the field $\nu(x), U_{C P}$ is the unitary operator of CP conjugation, $x \equiv\left(x^{0}, \vec{x}\right)$ and $x_{\mathrm{P}} \equiv\left(x^{0},-\vec{x}\right)$. The relation (A.17) can be written as

$$
U_{\mathrm{CP}} \mathcal{C} \bar{\nu}^{T}(x) U_{\mathrm{CP}}^{-1}=-\eta_{\mathrm{CP}}^{*} \gamma_{0} \mathcal{C} \bar{\nu}^{T}\left(x_{\mathrm{P}}\right) \text {. }
$$


Using the Majorana condition (A.2) one obtains

$$
U_{\mathrm{CP}} \nu(x) U_{\mathrm{CP}}^{-1}=-\eta_{\mathrm{CP}}^{*} \gamma_{0} \nu\left(x_{\mathrm{P}}\right) .
$$

From the comparison of the expressions (A.17) and (A.19) one finds that $\eta_{\mathrm{CP}}=-\eta_{\mathrm{CP}}^{*}$, which implies that

$$
\eta_{\mathrm{CP}}^{2}=-1
$$

and $\eta_{\mathrm{CP}}= \pm i$. As it can be seen from Eq.(A.18) written as

$$
U_{\mathrm{CP}} \nu^{c}(x) U_{\mathrm{CP}}^{-1}=-\eta_{\mathrm{CP}}^{*} \gamma_{0} \nu^{c}\left(x_{\mathrm{P}}\right) \text {, }
$$

in general the product of the CP parities of particles and antiparticles is equal to -1 . The relation (A.20) results from the fact that in the Majorana case there is no difference between particles and antiparticles.

\section{References}

[1] T. Kajita, Super-Kamiokande Coll., Talk presented at Neutrino '98 434], 1998.

[2] Y. Fukuda et al., Super-Kamiokande Coll., Phys. Rev. Lett. 81, 1562 (1998).

[3] W. Pauli, in Neutrino Physics, p. 1, edited by K. Winter, Cambridge University Press, 1991.

[4] E. Fermi, Ricerca Scient. 2, 12 (1933).

[5] E. Fermi, Z. Physik. 88, 161 (1934).

[6] F. Perrin, Comptes Rendues 197, 1625 (1933).

[7] O. Kofoed-Hansen, Phys. Rev. 71, 451 (1947); G.C. Hanna and B. Pontecorvo, Phys. Rev. 75, 451 (1949).

[8] L.L. Langer and R.J.D. Moffat, Phys. Rev. 88, 689 (1952).

[9] L. Landau, Nucl. Phys. 3, 127 (1957).

[10] T.D. Lee and C.N. Yang, Phys. Rev. 105, 1671 (1957).

[11] A. Salam, Il Nuovo Cim. 5, 299 (1957).

[12] M. Goldhaber, L. Grodzins and A.W. Sunyar, Phys. Rev. 109, 1015 (1958).

[13] V.L. Telegdi, History of Original Ideas and Basic Discoveries in Particle Physics, edited by H.B. Newman and T. Ypsilantis, p. 421, Plenum Press, New York, published in cooperation with NATO Scientific Affairs Division, 1996.

[14] L. Grénacs et al., Am. J. Phys. 50, 931 (1982).

[15] R.P. Feynman and M. Gell-Mann, Phys. Rev. 109, 193 (1958).

[16] E.C.G. Sudarshan and R. Marshak, Phys. Rev. 109, 1860 (1958).

[17] L.D. Landau, unpublished (1957), private communication of B. Pontecorvo to S.M. Bilenky.

[18] B. Pontecorvo, J. Exptl. Theoret. Phys. 33, 549 (1957) [Sov. Phys. JETP 6, 429 (1958)].

[19] B. Pontecorvo, J. Exptl. Theoret. Phys. 34, 247 (1958) [Sov. Phys. JETP 7, 172 (1958)].

[20] E. Majorana, Il Nuovo Cim. 14, 170 (1937). 
[21] Z. Maki, M. Nakagawa and S. Sakata, Prog. Theor. Phys. 28, 870 (1962).

[22] B. Pontecorvo, Zh. Eksp. Teor. Fiz. 53, 1717 (1967) [Sov. Phys. JETP 26, 984 (1968)].

[23] V. Gribov and B. Pontecorvo, Phys. Lett. B 28, 493 (1969).

[24] S.M. Bilenky and B. Pontecorvo, Phys. Lett. B 61, 248 (1976).

[25] H. Fritzsch and P. Minkowski, Phys. Lett. B 62, 72 (1976).

[26] S.M. Bilenky and B. Pontecorvo, Lett. Nuovo Cim. 17, 569 (1976).

[27] V. Barger, P. Langacker, J.P. Leveille and S. Pakvasa, Phys. Rev. Lett. 45, 692 (1980).

[28] S.M. Bilenky, J. Hošek and S.T. Petcov, Phys. Lett. B 94, 495 (1980).

[29] I.Yu. Kobzarev et al., Yad. Fiz. 32, 1590 (1980) [Sov. J. Nucl. Phys. 32, 823 (1980)].

[30] J. Schechter and J.W.F. Valle, Phys. Rev. D 22, 2227 (1980).

[31] R.N. Mohapatra and P.B. Pal, Massive Neutrinos in Physics and Astrophysics, World Scientific Lecture Notes in Physics, Vol. 41, World Scientific, Singapore, 1991.

[32] M. Gell-Mann, P. Ramond and R. Slansky, in Supergravity, p. 315, edited by F. van Nieuwenhuizen and D. Freedman, North Holland, Amsterdam, 1979.

[33] T. Yanagida, Proc. of the Workshop on Unified Theory and the Baryon Number of the Universe, KEK, Japan, 1979.

[34] R.N. Mohapatra and G. Senjanović, Phys. Rev. Lett. 44, 912 (1980).

[35] C. Caso et al., Particle Data Group, Eur. Phys. J. C 3, 1 (1998).

[36] R. Davis, Harmer and K.C. Hoffman, Phys. Rev. Lett. 21, 1205 (1968).

[37] R. Davis, Prog. Part. Nucl. Phys. 32, 13 (1994).

[38] B.T. Cleveland et al., Astrophys. J. 496, 505 (1998).

[39] K.S. Hirata et al., Kamiokande Coll., Phys. Rev. D 38, 448 (1988).

[40] K.S. Hirata et al., Kamiokande Coll., Phys. Rev. D 44, 2241 (1991).

[41] K.S. Hirata et al., Kamiokande Coll., Phys. Rev. Lett. 77, 1683 (1996).

[42] P. Anselmann et al., GALLEX Coll., Phys. Lett. B 285, 376 (1992).

[43] W. Hampel et al., GALLEX Coll., Phys. Lett. B 388, 384 (1996).

[44] A.I. Abazov et al., SAGE Coll., Phys. Rev. Lett. 67, 3332 (1991).

[45] D.N. Abdurashitov et al., SAGE Coll., Phys. Rev. Lett. 77, 4708 (1996).

[46] K. Inoue, Super-Kamiokande Coll., Talk presented at the $5^{\text {th }}$ International Workshop on Topics in Astroparticle and Underground Physics, Gran Sasso, Italy, September, 1997 (http://www-sk. icrr.u-tokyo.ac.jp/doc/sk/pub/pub_sk.html).

[47] Y. Fukuda et al., Super-Kamiokande Coll., Phys. Rev. Lett. 81, 1158 (1998).

[48] Y. Suzuki, Super-Kamiokande Coll., Talk presented at Neutrino '98 [434], 1998.

[49] Y. Fukuda et al., Kamiokande Coll., Phys. Lett. B 335, 237 (1994).

[50] R. Becker-Szendy et al., IMB Coll., Nucl. Phys. B (Proc. Suppl.) 38, 331 (1995).

[51] W.W.M. Allison et al., Soudan-2 Coll., Phys. Lett. B 391, 491 (1997).

[52] M. Ambrosio et al., MACRO Coll., Phys. Lett. B 434, 451 (1998). 
[53] C. Athanassopoulos et al., LSND Coll., Phys. Rev. Lett. 77, 3082 (1996).

[54] C. Athanassopoulos et al., LSND Coll., Phys. Rev. Lett. 81, 1774 (1998).

[55] V. Lobashov, Talk presented at Neutrino '98 434.

[56] C. Weinheimer, Talk presented at Neutrino '98 [434], 1998.

[57] E.W. Kolb and M.S. Turner, The Early Universe, Addison-Wesley, 1990.

[58] F. Boehm and P. Vogel, Physics of Massive Neutrinos, Cambridge University Press, Cambridge, 1987.

[59] B. Kayser, F. Gibrat-Debu and F. Perrier, The Physics of Massive Neutrinos, World Scientific Lecture Notes in Physics, Vol.25, World Scientific, Singapore, 1989.

[60] J.N. Bahcall, Neutrino Physics and Astrophysics, Cambridge University Press, 1989.

[61] C.W. Kim and A. Pevsner, Neutrinos in Physics and Astrophysics, Contemporary Concepts in Physics, Vol. 8, Harwood Academic Press, Chur, Switzerland, 1993.

[62] S.M. Bilenky and B. Pontecorvo, Phys. Rep. 41, 225 (1978).

[63] P.H. Frampton and P. Vogel, Phys. Rep. 82, 339 (1982).

[64] J.D. Vergados, Phys. Rep. 133, 1 (1986).

[65] S.M. Bilenky and S.T. Petcov, Rev. Mod. Phys. 59, 671 (1987).

[66] J.W.F. Valle, Prog. Part. Nucl. Phys. 26, 91 (1991).

[67] L. Oberauer and F. von Feilitzsch, Rept. Prog. Phys. 55, 1093 (1992).

[68] G. Gelmini and E. Roulet, Rept. Prog. Phys. 58, 1207 (1995).

[69] J. Brunner, Fortsch. Phys. 45, 343 (1997).

[70] K. Zuber, Phys. Rep. 305, 295 (1998).

[71] J.M. Conrad, Talk presented at the $29^{\text {th }}$ International Conference on High-Energy Physics (ICHEP 98), Vancouver, Canada, 23-29 July, 1998 (hep-ex/9811009).

[72] J.W.F. Valle, hep-ph/9809234 (1998).

[73] G. Raffelt, hep-ph/9902271 (1999).

[74] S.L. Glashow, Nucl. Phys. 22, 597 (1961).

[75] S. Weinberg, Phys. Rev. Lett. 19, 1264 (1967).

[76] A. Salam, Proc. of the $8^{\text {th }}$ Nobel Symposium on Elementary Particle Theory, Relativistic Groups and Analyticity, edited by N. Svartholm, 1969.

[77] R. Bertlmann and H. Pietschmann, Phys. Rev. D 15, 683 (1977); J. Ellis et. al., Ann. Rev. Nucl. Part. Sci. 32, 443 (1982).

[78] N. Cabibbo, Phys. Rev. Lett. 10, 531 (1963).

[79] M. Kobayashi and T. Maskawa, Prog. Theor. Phys. 49, 652 (1973).

[80] J. Schechter and J.W.F. Valle, Phys. Rev. D 24, 1883 (1981) [Erratum: D 25, 283 (1982)].

[81] S.T. Petcov, Yad. Fiz. 25, 641 (1977) [Sov. J. Nucl. Phys. 25, 340, 698(E) (1977)].

[82] T.P. Cheng and L.F. Li, Phys. Rev. D 16, 1425 (1977).

[83] B.W. Lee and R.E. Shrock, Phys. Rev. D 16, 1444 (1977).

[84] L.L. Chau and W.Y. Keung, Phys. Rev. Lett. 53, 1802 (1984). 
[85] C. Jarlskog, Phys. Rev. Lett. 55, 1039 (1985); Z. Phys. C 29, 491 (1985); C. Jarlskog and R. Stora, Phys. Lett. B 208, 268 (1988); C. Jarlskog, Proc. of CP Violation, edited by C. Jarlskog, Advanced Series in High Energy Physics Vol. 3, p. 3, World Scientific, Singapore, 1989.

[86] D.D. Wu, Phys. Rev. D 33, 860 (1986); I. Dunietz, O.W. Greenberg and D.D. Wu, Phys. Rev. Lett. 55, 2935 (1985).

[87] J.D. Bjorken and I. Dunietz, Phys. Rev. D 36, 2109 (1987); I. Dunietz, Ann. Phys. 184, 350 (1988).

[88] J. Schechter and J.W.F. Valle, Phys. Rev. D 21, 309 (1980).

[89] I. Schur, Am. J. Math. 67, 472 (1945).

[90] B. Zumino, J. Math. Phys. 3, 1055 (1962).

[91] G. Gelmini and M. Roncadelli, Phys. Lett. B 99, 411 (1981).

[92] H. Georgi et al., Nucl. Phys. B 193, 297 (1983).

[93] J.E. Kim, Phys. Rep. 150, 1 (1987).

[94] W. Grimus and H. Neufeld, Nucl. Phys. B 325, 18 (1989).

[95] L. Wolfenstein, Phys. Lett. B 107, 77 (1981).

[96] S.M. Bilenky, N.P. Nedelcheva and S.T. Petcov, Nucl. Phys. B 247, 61 (1984).

[97] B. Kayser, Phys. Rev. D 30, 1023 (1984).

[98] S.M. Bilenky and S.T. Petcov, Rev. Mod. Phys. 59, 671 (1987).

[99] W. Konetschny and W. Kummer, Phys. Lett. B 70, 433 (1977).

[100] A. Zee, Phys. Lett. B 93, 387 (1980).

[101] A. Zee, Phys. Lett. B 161, 141 (1985).

[102] A. Zee, Nucl. Phys. B 264, 99 (1986).

[103] M.B. Voloshin, Sov. J. Nucl. Phys. 48, 512 (1988).

[104] K.S. Babu and R.N. Mohapatra, Phys. Rev. D 43, 2278 (1991).

[105] K.S. Babu, Phys. Lett. B 203, 132 (1988).

[106] M. Doi et al., Phys. Lett. B 102, 323 (1981).

[107] P. Langacker et al., Nucl. Phys. B 282, 589 (1987).

[108] J.C. Pati and A. Salam, Phys. Rev. D 10, 275 (1975); R.N. Mohapatra and J.C. Pati, Phys. Rev. D 11, 566 and 2558 (1975).

[109] R.N. Mohapatra and G. Senjanović, Phys. Rev. D 23, 165 (1981).

[110] G. Ecker, W. Grimus and M. Gronau, Nucl. Phys. B 279, 429 (1987).

[111] G.C. Branco, W. Grimus and L. Lavoura, Nucl. Phys. B 312, 492 (1989).

[112] K. Kanaya, Prog. Theor. Phys. 64, 2278 (1980).

[113] J. Schechter and J.W.F. Valle, Phys. Rev. D 25, 774 (1982).

[114] D. Chang, R.N. Mohapatra and M.K. Parida, Phys. Rev. Lett. 52, 1072 (1984); D. Chang and R.N Mohapatra, Phys. Rev. D 32, 1248 (1985); D.G. Lee and R.N. Mohapatra, Phys. Lett. B 329, 463 (1994).

[115] S.A. Bludman, D.C. Kennedy and P.G. Langacker, Phys. Rev. D 45, 1810 (1992). 
[116] S.A. Bludman, D.C. Kennedy and P.G. Langacker, Nucl. Phys. B 374, 373 (1992).

[117] E. Witten, Phys. Lett. B 91, 81 (1980).

[118] J.C. Pati and A. Salam, Phys. Rev. Lett. 31, 661 (1973).

[119] H. Georgi and S.L. Glashow, Phys. Rev. Lett. 32, 438 (1974).

[120] P. Langacker, Phys. Rep. 72, 185 (1981).

[121] F. Wilczek and A. Zee, Phys. Rev. Lett. 43, 1571 (1979).

[122] S. Weinberg, Phys. Rev. Lett. 43, 1566 (1979).

[123] S. Weinberg, Phys. Rev. D 22, 1694 (1980).

[124] H.A. Weldon and A. Zee, Nucl. Phys. B 173, 269 (1980).

[125] S. Weinberg, Int. J. Mod. Phys. A 2, 301 (1987).

[126] E.Kh. Akhmedov, Z.G. Berezhiani and G. Senjanović, Phys. Rev. Lett. 69, 3013 (1992).

[127] E.Kh. Akhmedov, Z.G. Berezhiani, G. Senjanović and Z. Tao, Phys. Rev. D 47, 3245 (1993).

[128] L. Wolfenstein, Nucl. Phys. B 186, 147 (1981).

[129] M. Kobayashi, C.S. Lim and M.M. Nojiri, Phys. Rev. Lett. 67, 1685 (1991).

[130] C. Giunti, C.W. Kim and U.W. Lee, Phys. Rev. D 46, 3034 (1992).

[131] S. Nussinov, Phys. Lett. B 63, 201 (1976).

[132] B. Kayser, Phys. Rev. D 24, 110 (1981).

[133] C. Giunti, C.W. Kim and U.W. Lee, Phys. Rev. D 44, 3635 (1991).

[134] C. Giunti, C.W. Kim and U.W. Lee, Phys. Rev. D 45, 2414 (1992).

[135] C. Giunti, C.W. Kim and U.W. Lee, Phys. Lett. B 274, 87 (1992).

[136] C. Giunti, C.W. Kim, J.A. Lee and U.W. Lee, Phys. Rev. D 48, 4310 (1993).

[137] J. Rich, Phys. Rev. D 48, 4318 (1993).

[138] K. Kiers, S. Nussinov and N. Weiss, Phys. Rev. D 53, 537 (1996).

[139] W. Grimus and P. Stockinger, Phys. Rev. D 54, 3414 (1996).

[140] J.E. Campagne, Phys. Lett. B 400, 135 (1997).

[141] Y. Grossman and H.J. Lipkin, Phys. Rev. D 55, 2760 (1997).

[142] K. Kiers and N. Weiss, Phys. Rev. D 57, 3091 (1998).

[143] C. Giunti, C.W. Kim and U.W. Lee, Phys. Lett. B 421, 237 (1997).

[144] C. Giunti and C.W. Kim, Phys. Rev. D 58, 017301 (1998).

[145] W. Grimus, P. Stockinger and S. Mohanty, Phys. Rev. D 59, 013011 (1998).

[146] L. Stodolsky, Phys. Rev. D 58, 036006 (1998).

[147] E.J. Chun, C.W. Kim and U.W. Lee, Phys. Rev. D 58, 093003 (1998).

[148] Y. Koide, Phys. Rev. D 57, 5836 (1998).

[149] Y. Koide and H. Fusaoka, Phys. Rev. D 59, 053004 (1998).

[150] S.M. Bilenky and C. Giunti, Phys. Lett. B 300, 137 (1993).

[151] N. Cabibbo, Phys. Lett. B 72, 333 (1978). 
[152] D. Orestano, NOMAD Coll., Talk presented at the CAPP 98 Workshop, CERN, June, 1998 435].

[153] F. Dydak et al., CDHS Coll., Phys. Lett. B 134, 281 (1984).

[154] N. Ushida et al., Phys. Rev. Lett. 57, 2897 (1986).

[155] P. Vilain et al., CHARM II Coll., Z. Phys. C 64, 539 (1994).

[156] K.S. McFarland et al., CCFR Coll., Phys. Rev. Lett. 75, 3993 (1995).

[157] CHORUS Coll., Talk presented at the $29^{\text {th }}$ International Conference on High-Energy Physics (ICHEP 98), Vancouver, Canada, 23-29 July 1998 (preprint hep-ex/9807024).

[158] J. Altegoer et al., NOMAD Coll., Phys. Lett. B 431, 219 (1998).

[159] M. Apollonio et al., CHOOZ Coll., Phys. Lett. B 420, 397 (1998).

[160] G. Zacek et al., Phys. Rev. D 34, 2621 (1986).

[161] G.S. Vidyakin et al., JETP Lett. 59, 390 (1994).

[162] B. Achkar et al., Bugey Coll., Nucl. Phys. B 434, 503 (1995).

[163] L. Wolfenstein, Phys. Rev. D 17, 2369 (1978); ibid. 20, 2634 (1979).

[164] S.P. Mikheyev and A.Yu. Smirnov, Yad. Fiz. 42, 1441 (1985) [Sov. J. Nucl. Phys. 42, 913 (1985)]; Il Nuovo Cim. C 9, 17 (1986); Zh. Eksp. Teor. Fiz. 91, 7 (1986) [Sov. Phys. JETP 64, 4 (1986)].

[165] V. Barger, K. Whisnant, S. Pakvasa and R.J.N. Phillips, Phys. Rev. D 22, 2718 (1980).

[166] S.P. Rosen and J.M. Gelb, Phys. Rev. D 34, 969 (1986).

[167] H.A. Bethe, Phys. Rev. Lett. 56, 1305 (1986).

[168] A. Halprin, Phys. Rev. D 34, 3462 (1986); P.D. Mannheim, Phys. Rev. D 37, 1935 (1988).

[169] W. Grimus and T. Scharnagl, Mod. Phys. Lett. A 8, 1943 (1993).

[170] P.B. Pal and T.N. Pham, Phys. Rev. D 40, 259 (1989).

[171] J. Liu, Phys. Rev. D 45, 1428 (1992).

[172] P.B. Pal, Int. J. Mod. Phys. A 22, 5387 (1992).

[173] C. Giunti, C.W. Kim and W.P. Lam, Phys. Rev. D 43, 164 (1991).

[174] J.N. Bahcall and R. Ulrich, Rev. Mod. Phys. 60, 297 (1988).

[175] J.N. Bahcall and M.H. Pinsonneault, Rev. Mod. Phys. 67, 781 (1995).

[176] L.L. Foldy and S.A. Wouthuysen, Phys. Rev. 78, 29 (1950).

[177] P.G. Langacker et al., Nucl. Phys. B 282, 589 (1987).

[178] S.P. Mikheyev and A.Yu. Smirnov, Prog. Part. Nucl. Phys. 23, 41 (1989).

[179] L. Stodolsky, Phys. Rev. D 36, 2273 (1987).

[180] H. Nunokawa, A. Rossi, V.B. Semikoz and J.W.F. Valle, Nucl. Phys. B 472, 495 (1996); A.B. Balantekin, J.M. Fetter and F.N. Loreti, Phys. Rev. D 54, 3941 (1996); C.P. Burgess and D. Michaud, Ann. Phys. 256, 1 (1997).

[181] P. Bamert, C.P. Burgess and D. Michaud, Nucl. Phys. B 513, 319 (1998).

[182] A.J. Baltz and J. Weneser, Phys. Rev. D 37, 3364 (1988).

[183] T.K. Kuo and J. Pantaleone, Rev. Mod. Phys. 61, 937 (1989).

[184] W.C. Haxton, Ann. Rev. Astron. Astrophys. 33, 459 (1995). 
[185] L.I. Schiff, Quantum Mechanics, McGaw-Hill, Singapore, 1987.

[186] V. A. Naumov, Zh. Eksp. Teor. Fiz. 101, 3 (1992) [Sov. Phys. JETP. 74, 1 (1992)]; Int. J. Mod. Phys. D 1, 379 (1992); Phys. Lett. B323, 351 (1994).

[187] S.J. Parke, Phys. Rev. Lett. 57, 1275 (1986).

[188] W.C. Haxton, Phys. Rev. Lett. 57, 1271 (1986); Phys. Rev. D 35, 2352 (1987).

[189] T.K. Kuo and J. Pantaleone, Phys. Rev. D 39, 1930 (1989).

[190] M. Born and V. Fock, Z. Phys. 51, 165 (1928).

[191] E. Lisi and D. Montanino, Phys. Rev. D 56, 1792 (1997).

[192] C. Giunti, C.W. Kim and M. Monteno, Nucl. Phys. B 521, 3 (1998).

[193] S.T. Petcov, Phys. Lett. B 200, 373 (1988); Phys. Lett. B 214, 139 (1988).

[194] S.T. Petcov and J. Rich, Phys. Lett. B 224, 426 (1989).

[195] S.T. Petcov, Phys. Lett. B 191, 299 (1987).

[196] C. Zener, Proc. Roy. Soc. Lon. A 137, 696 (1932).

[197] L. Landau, Phys. Z. Sov. 2, 46 (1932).

[198] A. Joye and Ch.-E. Pfister, preprint math-ph/9807031 (1998).

[199] P. Pizzochero, Phys. Rev. D 36, 2293 (1987).

[200] A.B. Balantekin and J.F. Beacom, Phys. Rev. D 54, 6323 (1996); A.B. Balantekin, J.F. Beacom and J.B. Fetter, Phys. Lett. B 427, 317 (1998).

[201] T. Kaneko, Prog. Theor. Phys. 78, 532 (1987); S. Toshev, Phys. Lett. B 196, 170 (1987); M. Ito, T. Kaneko and M. Nakagawa, Prog. Theor. Phys. 79, 13 (1988).

[202] D. Nötzold, Phys. Rev. D 36, 1625 (1987).

[203] P.I. Krastev and S.T. Petcov, Phys. Lett. B 207, 64 (1988) [Erratum: B 214, 661 (1988)].

[204] V.F. Hess, Phys. Zeitschr. 13, 1084 (1913).

[205] A. Pais, Inward bound, Oxford University Press, Oxford, 1994.

[206] L.V. Volkova, Jad. Fiz. 31, 784 (1980) [Sov. J. Nucl. Phys. 31, 1510 (1980)].

[207] K. Mitsui, Y. Minorikawa and H. Komori, Il Nuovo Cim. C 9, 995 (1986).

[208] E.V. Bugaev and V.A. Naumov, Izv. Akad. Nauk SSSR, Ser. Fiz. 50, 2239 (1986) [Bull. Acad. of Sci. of the USSR, Phys. Ser. 50, 156 (1986)].

[209] E.V. Bugaev and V.A. Naumov, Yad. Fiz. 45, 1380 (1987) [Sov. J. Nucl. Phys. 45, 857 (1987)].

[210] A.V. Butkevich, L.G. Dedenko and I.M. Zeleznykh, Yad. Fiz. 50, 142 (1989) [Sov. J. Nucl. Phys. 50, 90 (1989)].

[211] G. Barr, T.K. Gaisser and T. Stanev, Phys. Rev. D 39, 3532 (1989).

[212] H. Lee and Y.S. Koh, Il Nuovo Cim. 105 B, 883 (1989).

[213] E.V. Bugaev and V.A. Naumov, Phys. Lett. B 232, 391 (1989).

[214] M. Honda, K. Kasahara, K. Hidaka and S. Midorikawa, Phys. Lett. B 248, 193 (1990).

[215] M. Honda, T. Kajita, K. Kasahara and S. Midorikawa, Phys. Rev. D 52, 4985 (1995).

[216] V. Agrawal, T.K. Gaisser, P. Lipari and T. Stanev, Phys. Rev. D 53, 1314 (1996). 
[217] T.K. Gaisser, M. Honda, K. Kasahara, S. Midorikawa, V. Naumov and T. Stanev, Phys. Rev. D 54, 5578 (1996).

[218] P. Lipari, T. Stanev and T.K. Gaisser, Phys. Rev. D 58, 073003 (1998).

[219] T.K. Gaisser, Cosmic Rays and Particle Physics, Cambridge University Press, Cambridge, 1990.

[220] V.A. Dogiel, V.S. Berezinsky, S.V. Bulanov and V.S. Ptuskin, Astrophysics of Cosmic Rays, edited by V.L. Ginzburg, North-Holland, Amsterdam, 1990.

[221] P. Lipari, Astropart. Phys. 1, 195 (1993).

[222] A. Misaki, V.A. Naumov, T.S. Sinegovskaya and S.I. Sinegovsky, Proc. of the 25th International Cosmic Ray Conference, Durban, South Africa, edited by M. S. Potgieter et al., p. 129, 1997.

[223] V.A. Naumov, T.S. Sinegovskaya and S.I. Sinegovsky, Il Nuovo Cim. A 111, 129 (1998).

[224] J.A. Simpson, Ann. Rev. Nucl. Part. Sci. 33, 323 (1983).

[225] W.R. Webber and J.A. Lezniak, Astrophys. Space Sci. 30, 361 (1974).

[226] E.-S. Seo et al., Proc. of the 22nd International Cosmic Ray Conference, Dublin, Ireland, edited by M. Cawley et. al., Vol. 2, p. 627, Reprint Ltd., Dublin, Ireland, 1991.

[227] E.V. Bugaev and V.A. Naumov, Proc. of the Second International Symposium Underground Physics'87, Baksan Valley, August 1987, edited by G. V. Domogatsky et al., Nauka, Moscow, p. 255, 1988.

[228] P. Lipari and T. Stanev, Proc. of the 24th International Cosmic Ray Conference, Rome, Vol. 1, p. 694, 1995.

[229] H. Lee and S.A. Bludman, Phys. Rev. D 37, 122 (1988).

[230] J.L. Osborne, S.S. Said and A.E. Wolfendale, Proc. Phys. Soc. 86, 93 (1965).

[231] T.K. Gaisser, Proc. of Neutrino '96, Helsinki, June 1996, edited by K. Enqvist et al., p. 211, World Scientific, Singapore, 1997.

[232] T.K. Gaisser and T. Stanev, Phys. Rev. D 57, 1977 (1998).

[233] T.K. Gaisser, Phil. Trans. Roy. Soc. Lon. A 346, 75 (1994).

[234] M.A. Markov, Proc. of 1960 Annual International Conference on High Energy Physics, Rochester, edited by E.C.G. Sudarshan et al., 1960.

[235] K. Greisen, Ann. Rev. Nucl. Sci. 10, 63 (1960).

[236] C.V. Achar et al., Phys. Lett. 18, 196 and 19, 78 (1965).

[237] F. Reines et al., Phys. Rev. Lett. 15, 429 (1965).

[238] M.A. Markov and I.M. Zhelenykh, Nucl. Phys. 27, 385 (1961).

[239] G.T. Zatsepin and V.A. Kuzmin, Sov. J. Nucl. Phys. 14, 1294 (1962).

[240] R. Cowsik, Y. Pal and S.N. Tandon, Proc. Ind. Acad. Sci. 63, 217 (1966).

[241] E.W. Beier and E.D. Frank, Phil. Trans. Roy. Soc. Lon. A 346, 63 (1994).

[242] K.S. Hirata et al., Kamiokande Coll., Phys. Lett. B 205, 416 (1988).

[243] K.S. Hirata et al., Kamiokande Coll., Phys. Lett. B 280, 146 (1992).

[244] R. Becker-Szendy et al., IMB Coll., Phys. Rev. Lett. 69, 1010 (1992).

[245] R. Clark et al., IMB Coll., Phys. Rev. Lett. 79, 345 (1997).

[246] K. Daum et al., Fréjus Coll., Z. Phys. C 66, 417 (1995). 
[247] M. Aglietta et al., NUSEX Coll., Europhys. Lett. 8, 611 (1989).

[248] T.K. Gaisser, F. Halzen and T. Stanev, Phys. Rep. 258, 174 (1995).

[249] W. Frati et al., Phys. Rev. D 48, 1140 (1993).

[250] M.M. Boliev et al., Proc. of the 24th International Cosmic Ray Conference, ICRC '95, Rome, Vol. 1, p. 686, Arti Grafiche Editoriali, Urbino, Italy, 1995; ibidem, p. 722; S.P. Mikheyev, Proc. of the $8^{\text {th }}$ Rencontres de Blois on Neutrinos, Dark Matter, and the Universe, Blois, France, 1996; M.M. Boliev et al., Proc. of the International Workshop on Aspects of Dark Matter in Astrophysics and Particle Physics, DARK '96, Heidelberg, Germany, 1996.

[251] Y. Totsuka et al., Kamiokande Coll., Nucl. Phys. B 31 (Proc. Suppl.), 428 (1993).

[252] S. Hatakeyama et al., Kamiokande Coll., Phys. Rev. Lett. 81, 2016 (1998).

[253] P. Lipari, M. Lusignoli and F. Sartogo, Phys. Rev. Lett. 74, 4384 (1995).

[254] T.J. Haines et al., IMB Coll., Phys. Rev. Lett. 57, 1986 (1986).

[255] E. Kearns, Nucl. Phys. B (Proc. Suppl.) 70, 315 (1999) (hep-ex/9803007).

[256] T. Kafka, Nucl. Phys. B (Proc. Suppl.) 70, 340 (1999) (hep-ex/9712281).

[257] M.C. Gonzalez-Garcia et al., Phys. Rev. D 58, 033004 (1998).

[258] M.C. Gonzalez-Garcia et al., preprint FTUV/98-56, IFIC/98-57, hep-ph/9807305 (1998).

[259] R. Foot, R.R. Volkas and O. Yasuda, Phys. Rev. D 58, 013006 (1998).

[260] O. Yasuda, Phys. Rev. D 58, 091301 (1998).

[261] G.L. Fogli, E. Lisi, A. Marrone and G. Scioscia, Phys. Rev. D 59, 033001 (1999).

[262] G.L. Fogli, E. Lisi and D. Montanino, Phys. Rev. D 49, 3626 (1994).

[263] G.L. Fogli, E. Lisi and D. Montanino, Astropart. Phys. 4177 (1995).

[264] G.L. Fogli and E. Lisi, Phys. Rev. D 52, 2775 (1995).

[265] G.L. Fogli, E. Lisi, D. Montanino and G. Scioscia, Phys. Rev. D 55, 4385 (1997).

[266] G.L. Fogli, E. Lisi, A. Marrone and D. Montanino, Phys. Lett. B 425, 341 (1998).

[267] J.N. Flanagan, J.G. Learned and S. Pakvasa, Phys. Rev. D 57, R2649 (1998).

[268] J. Bunn, R. Foot and R. Volkas, Phys. Lett. B 413, 109 (1997).

[269] R. Foot, R. Volkas and O. Yasuda, Phys. Rev. D 58, 013006 (1998).

[270] R. Foot, C.N. Leung and O. Yasuda, Phys. Lett. B 443, 185 (1998).

[271] Y. Suzuki, Proc. of Neutrino 96, Helsinki, June 1996, edited by K. Enqvist et. al., p. 237, World Scientific, Singapore, 1997.

[272] F. Vissani and A.Yu. Smirnov, Phys. Lett. B 432, 376 (1998).

[273] A. McDonald, SNO Coll., Talk presented at Neutrino '98 [434, 1998.

[274] Y. Fukuda et al., Kamiokande Coll., Phys. Lett. B 388, 397 (1996).

[275] K.R.S. Balaji, G. Rajasekaran and S. Uma Sankar, preprint hep-ph/9806256 (1998).

[276] F. Vissani, Talk presented at the Ringberg Euroconference New Trends in Neutrino Physics, Tegernsee, Germany, May 1998, preprint DESY-98-135, 1998.

[277] J.G. Learned, S. Pakvasa and J.L. Stone, Phys. Lett. B 435, 131 (1998).

[278] E. Akhmedov, P. Lipari and M. Lusignoli, Phys. Lett. B 300, 128 (1993). 
[279] G.L. Fogli, E. Lisi and A. Marrone, Phys. Rev. D 57, 5893 (1998).

[280] Q.Y. Liu and A.Yu. Smirnov, Nucl. Phys. B 524, 505 (1998).

[281] V.K. Ermilova, V.A. Tsarev and V.A. Chechin, Kr. Soob., Fiz. [Short Notices of the Lebedev Institute] 5, 26 (1986); E.Kh. Akhmedov, Yad. Fiz. 47, 475 (1988) [Sov. J. Nucl. Phys. 47, 301 (1988)].

[282] Q.Y. Liu, S.P. Mikheyev and A.Yu. Smirnov, hep-ph/9803415(1998).

[283] S.T. Petcov, Phys. Lett. B 434, 321 (1998).

[284] E.Kh. Akhmedov, Nucl. Phys. B 538, 25 (1999).

[285] M. Chizhov, M. Maris and S.T. Petcov, hep-ph/9810501 (1998).

[286] E.Kh. Akhmedov, A. Dighe, P. Lipari and A.Yu. Smirnov, hep-ph9808270 (1998).

[287] P. Lipari and M. Lusignoli, Phys. Rev. D 57, 3842 (1998).

[288] F. Boehm et al., The Palo Verde experiment, 1996 (http://www.cco.caltech.edu/ ${ }^{\sim}$ songhoon/PaloVerde/Palo-Verde.html).

[289] F. Boehm et al., Palo Verde Coll., Nucl. Phys. B (Proc. Suppl.) 70, 191 (1999).

[290] F. Suekane, preprint TOHOKU-HEP-97-02 (1997).

[291] A. Suzuki, Talk presented at NOW'98 [376], 1998.

[292] S. Schönert et al., Nucl. Phys. B (Proc. Suppl.) 70, 195 (1999).

[293] D. Ayres et al., MINOS Coll., report NUMI-L-63 (1995).

[294] P. Cennini et al., ICARUS Coll., report LNGS-94/99-I (1994).

[295] H. Shibuya et al., OPERA Coll., report CERN-SPSC-97-24 (1997).

[296] M. Ambrosio et al., NOE Coll., Nucl. Instr. Meth. A 363, 604 (1995); G.C. Barbarino et al., NOE Coll., report INFN/AE-96/11 (1996).

[297] T. Ypsilantis et al., AQUA-RICH Coll., report CERN-LAA/96-13 (1996).

[298] G. Baldini et al., NICE Coll., report LNGS-LOI 98/13 (1998).

[299] P. Picchi and F. Pietropaolo, Talk presented at Neutrino '98 [434 (preprint hep-ph/9812222 (1998).

[300] M. Aglietta et al., preprint CERN-SPSC-98-28 (1998).

[301] G. Battistoni and P. Lipari, to appear in the Proc. of the 1998 Vulcano Workshop on Frontier Objects in Astrophysics and Particle Physics, 1998 (hep-ph/9807475).

[302] A. Geiser, Contribution to NOW'98 [376], 1998 (hep-ph/9810493).

[303] J.N. Bahcall and M.H. Pinsonneault, Rev. Mod. Phys. 64, 885 (1992).

[304] J.N. Bahcall, S. Basu and M.H. Pinsonneault, Phys. Lett. B 433, 1 (1998).

[305] S. Turck-Chièze, S. Cahen, M. Cassé and C. Doom, Astrophys. J. 335, 415 (1988).

[306] S. Turck-Chièze and I. Lopes, Astrophys. J. 408, 347 (1993).

[307] S. Turck-Chièze et al., Phys. Rep. 230, 57 (1993).

[308] V. Castellani, S. Degl'Innocenti and G. Fiorentini, Astron. Astrophys. 271, 601 (1993).

[309] V. Castellani et al., Phys. Lett. B 324, 425 (1994).

[310] F. Ciacio, S. Degl'Innocenti and B. Ricci et al., Astron. Astrophys. Suppl. Ser. 123, 449 (1997). 
[311] M. Koshiba, Phys. Rep. 220, 229 (1992).

[312] V. Castellani et al., Phys. Rep. 281, 309 (1997).

[313] V.S. Berezinsky, Invited lecture at the $25^{\text {th }}$ International Cosmic Ray Conference, Durban, 28 July - 8 August, 1997 astro-ph/9710126).

[314] J.N. Bahcall, Lectures presented at the XXV SLAC Summer Institute on Particle Physics, Physics of Leptons, August 4-15, 1997 (hep-ph/9711358).

[315] J.N. Bahcall, Talk presented at Neutrino '98 [434, 1998 (hep-ph/9808162).

[316] A.Yu. Smirnov, Talk presented at Neutrino '98 [434, 1998 (hep-ph/9809481).

[317] J.N. Bahcall et al., Phys. Rev. C 54, 411 (1996).

[318] J.N. Bahcall, Phys. Rev. C 56, 3391 (1997).

[319] J.N. Bahcall, Phys. Rev. D 49, 3923 (1994).

[320] J.N. Bahcall, Phys. Rev. D 44, 1644 (1991).

[321] J.N. Bahcall and P.I. Krastev, Phys. Rev. D 53, 4211 (1996).

[322] T. Kirsten, GNO Coll., Talk presented at Neutrino '98 [434], 1998.

[323] V.N. Gavrin, SAGE Coll., Talk presented at Neutrino '98 [434, 1998.

[324] B. Pontecorvo, Chalk River Report PD-205 (1946).

[325] L.W. Alvarez, University of California Radiation Laboratory Report UCRL-328 (1949).

[326] W. Hampel et al., GALLEX Coll., Phys. Lett. B 420, 114 (1998).

[327] W. Hampel et al., GALLEX Coll., preprint DAPNIA-SPP-98-07 (1998).

[328] M. Cribier et al., GALLEX Coll., Nucl. Phys. B (Proc. Suppl.) 70, 284 (1999).

[329] J.N. Bahcall, P.I. Krastev and A.Yu. Smirnov, Phys. Rev. D 58, 096016 (1998).

[330] J.N. Bahcall, M.H. Pinsonneault, S.Basu and J. Christensen-Dalsgaard, Phys. Rev. Lett. 78, 171 (1997).

[331] B. Ricci et al., Phys. Lett. B 407, 155 (1997).

[332] D. Gough, Talk presented at Neutrino '98 [434], 1998.

[333] A.S. Brun, S. Turck-Chièze and P Morel, preprint astro-ph/9806272 (1998).

[334] M. Spiro and D. Vignaud, Phys. Lett. B 242, 279 (1990).

[335] V. Castellani, S. Degl'Innocenti and G. Fiorentini, Phys. Lett. B 303, 68 (1993).

[336] N. Hata, S.A. Bludman and P. Langacker et al., Phys. Rev. D 49, 3622 (1994).

[337] V.S. Berezinsky, Comm. Nucl. Part. Phys. 21, 249 (1994).

[338] J.N. Bahcall, Phys. Lett. B 338, 276 (1994).

[339] S. Degl'Innocenti, G. Fiorentini and M. Lissia, Nucl. Phys. B (Proc. Suppl.) 43, 66 (1995).

[340] S. Parke, Phys. Rev. Lett. 74, 839 (1995).

[341] K.H. Heeger and R.G.H. Robertson, Phys. Rev. Lett. 77, 3270 (1996).

[342] N. Hata and P. Langacker, Phys. Rev. D 56, 6107 (1997).

[343] H. Minakata and H. Nunokawa, Phys. Rev. D 59, 073004 (1999).

[344] G.L. Fogli, E. Lisi and D. Montanino, Astropart. Phys. 9, 119 (1998). 
[345] M. Narayan, M.V.N. Murthy, G. Rajasekaran and S. Uma Sankar, Phys. Rev. D 53, 2809 (1996).

[346] S. Goswami, K. Kar and A. Raychaudhuri, Int. J. Mod. Phys. A 12, 781 (1997).

[347] G.L. Fogli, E. Lisi and D. Montanino, Phys. Rev. D 54, 2048 (1996).

[348] P. Osland and G. Vigdel, Phys. Lett. B 438, 129 (1998).

[349] P.I. Krastev and S.T. Petcov, Phys. Rev. D 53, 1665 (1996).

[350] P.I. Krastev, Q.Y. Liu and S.T. Petcov, Phys. Rev. D 54, 7057 (1996).

[351] M. Maris and S.T. Petcov, Phys. Rev. D 56, 7444 (1997); Phys. Rev. D 58, 113008 (1998).

[352] L. Oberauer, Borexino Coll., Talk presented at Neutrino '98 [434, 1998.

[353] Y. Suzuki (SuperKamiokande), Proc. of the Fourth International Solar Neutrino Conference, Heidelberg, Germany, 8-11 April 1997, edited by W. Hampel, p. 163, Max-Planck-Institut für Kernphysik, 1997; R. Meijer Drees (SNO), ibid., p. 210; F. von Feilitzsch (Borexino), ibid., p. 192; E. Bellotti (GNO), ibid., p. 173; K. Lande (Homestake Iodine), ibid., p. 228; C. Tao (HELLAZ), ibid., p. 238; A.V. Kopylov (Lithium), ibid., p. 263; Yu.G. Zdesenko (Xenon), ibid., p. 283; P. Cennini et al. (ICARUS), LNGS-94/99-I, May 1994; T.J. Bowels (GaAs), Proc. of Neutrino 96, Helsinki, Finland, 13-19 June 1996, edited by K. Enqvist et al., p. 83, World Scientific, Singapore, 1997.

[354] R.E. Lanou, Talk presented at Neutrino '98 [434], 1998 (hep-ex/9808033).

[355] S.M. Bilenky and C. Giunti, Phys. Lett. B 311, 179 (1993).

[356] S.M. Bilenky and C. Giunti, Phys. Lett. B 320, 323 (1994); Astrop. Phys. 2, 353 (1994); Z. Phys. C 68, 495 (1995).

[357] W.K. Kwong and S.P. Rosen, Phys. Rev. D 51, 6159 (1995).

[358] W.K. Kwong and S.P. Rosen, Phys. Rev. D 54, 2043 (1996).

[359] F.L. Villante, G. Fiorentini and E. Lisi, Phys. Rev. D 59, 013006 (1999).

[360] J.N. Bahcall and P.I. Krastev, Phys. Rev. C 55, 929 (1997).

[361] J.N. Bahcall and P.I. Krastev, Phys. Rev. C 56, 2839 (1997).

[362] G.L. Fogli, E. Lisi and D. Montanino, Phys. Lett. B 434, 333 (1998).

[363] B. Faid, G.L. Fogli and E. Lisi D. Montanino, Astropart. Phys. 10, 93 (1999).

[364] SNO WWW page: http://www.sno.queensu.ca.

[365] Borexino WWW pages: http://almime.mi.infn.it// http://pupgg.princeton.edu/ ${ }^{\sim}$ borexino/welcome.html.

[366] C. Athanassopoulos et al., LSND Coll., Phys. Rev. Lett. 75, 2650 (1995).

[367] C. Athanassopoulos et al., LSND Coll., Phys. Rev. C 54, 2685 (1996).

[368] C. Athanassopoulos et al., LSND Coll., Nucl. Instrum. Methods Phys. Res. A 388, 149 (1997).

[369] C. Athanassopoulos et al., LSND Coll., preprint nucl-ex/9706006 (1997).

[370] D.H. White, Talk presented at Neutrino '98 434, 1998.

[371] LSND WWW page: http://www.neutrino.lanl.gov/LSND/.

[372] L.A. Ahrens et al., BNL E734 Coll., Phys. Rev. D 36, 702 (1987).

[373] L. Borodovsky et al., BNL E776 Coll., Phys. Rev. Lett. 68, 274 (1992).

[374] A. Romosan et al., CCFR Coll., Phys. Rev. Lett. 78, 2912 (1997). 
[375] B. Zeitnitz, KARMEN Coll., Talk presented at Neutrino '98 434, 1998.

[376] Neutrino Oscillation Workshop NOW'98, Amsterdam, 7-9 September 1998; WWW page: http:// www.nikhef.nl/pub/conferences/now98/.

[377] G. Drexlin et al., KARMEN Coll., Nucl. Instrum. Methods Phys. Res. A 289, 490 (1990).

[378] G. Drexlin et al., KARMEN Coll., Prog. Part. Nucl. Phys. 32, 375 (1994).

[379] B. Armbruster, KARMEN Coll., Talk presented at the XXXIII ${ }^{\text {nd }}$ Rencontres de Moriond: Electroweak Interactions and Unified Theories, Les Arcs 1800 (France), March 14-21, 1998 [382].

[380] K. Eitel and B. Zeitnitz, KARMEN Coll., Contribution to Neutrino '98 [434], 1998 hepex/9809007).

[381] B. Armbruster, KARMEN Coll., Contribution to NOW'98 [376], 1998 (see also 382]).

[382] KARMEN WWW page: http://www-ik1.fzk.de/www/karmen/karmen_e.htm].

[383] G.J. Feldman and R.D. Cousins, Phys. Rev. D 57, 3873 (1998).

[384] R.D. Cousins, Am. J. Phys. 63, 398 (1995).

[385] C. Giunti, Phys. Rev. D 59, 053001 (1998).

[386] C. Giunti, in the Summary of the Phenomenology Working Group at NOW'98 376], 1998.

[387] Booster Neutrino Experiment (BooNE), http://nu1.lampf.lanl.gov/BooNE.

[388] I-216 $\nu_{\mu} \rightarrow \nu_{e}$ proposal at CERN, http://chorus01.cern.ch/ pzucchel/loi/.

[389] Oak Ridge Large Neutrino Detector, http://www.phys.subr.edu/orland/.

[390] NESS: Neutrinos at the European Spallation Source, http://www.isis.rl.ac.uk/ess/neut\%5Fess.htm.

[391] C.Y. Cardall and G.M. Fuller, Phys. Rev. D 53, 4421 (1996).

[392] K. Kang, J.E. Kim and P. Ko, Z. Phys. C 72, 671 (1996).

[393] A. Acker and S. Pakvasa, Phys. Lett. B 397, 209 (1997).

[394] T. Teshima, T. Sakai and O. Inagaki, preprint hep-ph/9801276 (1998).

[395] R.P. Thun and S. McKee, Phys. Lett. B 439, 123 (1998).

[396] G. Barenboim and F. Scheck, Phys. Lett. B 440, 332 (1998).

[397] G. Conforto, M. Barone and C. Grimani, Phys. Lett. B 447, 122 (1998).

[398] G.L. Fogli, E. Lisi, D. Montanino and G. Scioscia, Phys. Rev. D 56, 4365 (1997).

[399] G.L. Fogli, in the Summary of the Phenomenology Working Group at NOW'98 [376], 1998.

[400] R. Foot, Mod. Phys. Lett. A 9, 169 (1994); R. Foot and R.R. Volkas, Phys. Rev. D 52, 6595 (1995); Z. Berezhiani and R.N. Mohapatra, ibid 52, 6607 (1995); J. Bunn, R. Foot and R.R. Volkas, Phys. Lett. B 413, 109 (1997); B. Brahmachari and R.N. Mohapatra, Phys. Lett. B 437, 100 (1998); J.P. Bowes and R.R. Volkas, J. Phys. G 24, 1249 (1998); M. Collie and R. Foot, Phys. Lett. B 432, 134 (1998); R.R. Volkas and Y.Y.Y. Wong, Phys. Rev. D 58, 113001 (1998); D. Suematsu, Prog. Theor. Phys. 99, 483 (1998); hep-ph/9805305; W. Krolikowski, Acta Phys. Polon. B 29, 629 (1998); ibid. 29, 755 (1998); hep-ph/9803323; hep-ph/9808207; hep-ph/9808307; Y. Koide and H. Fusaoka, Phys. Rev. D 59, 053004 (1999); A. Geiser, Phys. Lett. B 444, 358 (1999).

[401] A. Geiser, in the Summary of the Phenomenology Working Group at NOW'98 [376], 1998.

[402] A. De Rujula et al., Nucl. Phys. B 168, 54 (1980). 
[403] V. Barger and K. Whisnant, Phys. Lett. B 209, 365 (1988).

[404] S.M. Bilenky, M. Fabbrichesi and S.T. Petcov, Phys. Lett. B 276, 223 (1992).

[405] S.M. Bilenky, C. Giunti and C.W. Kim, Astrop. Phys. 4, 241 (1996).

[406] X. Shi and D.N. Schramm, Phys. Lett. B 283, 305 (1992).

[407] S.M. Bilenky and C. Giunti, Phys. Lett. B 444, 379 (1998).

[408] R. Svoboda, Super-Kamiokande Coll., Talk presented at the Conference on Solar Neutrinos: News About SNUs, Santa Barbara, California, 2-6 December, 1997 (http://www.itp.ucsb.edu/online/$\mathrm{snu}$ ).

[409] M.C. Gonzalez-Garcia et al., Phys. Rev. D 58, 033004 (1998).

[410] F. Vissani, preprint hep-ph/9708483 (1997).

[411] V. Barger, S. Pakvasa, T.J. Weiler and K. Whisnant, Phys. Lett. B 437, 107 (1998); A.J. Baltz, A.S. Goldhaber and M. Goldhaber, Phys. Rev. Lett. 81, 5730 (1998); Y. Nomura and T. Yanagida, Phys. Rev. D 59, 017303 (1999); G. Altarelli and F. Feruglio, Phys. Lett. B 439, 112 (1998); M. Tanimoto, Phys. Rev. D 59, 017304 (1999); E. Ma, Phys. Lett. B 442, 238 (1998); N. Haba, Phys. Rev. D 59, 035011 (1999); H. Fritzsch and Z.Z. Xing, Phys. Lett. B 440, 313 (1998); M. Jeżabek and Y. Sumino, Phys. Lett. B 440, 327 (1998); H. Georgi and S.L. Glashow, hep-ph/9808293; S. Davidson and S.F. King, Phys. Lett. B 445, 191 (1998); R.N. Mohapatra and S. Nussinov, Phys. Lett. B 441, 299 (1998); hep-ph/9809415; G. Altarelli and F. Feruglio, JHEP 9811, 021 (1998); C. Jarlskog, M. Matsuda, S. Skadhauge and M. Tanimoto, hep-ph/9812282.

[412] C. Giunti, Phys. Rev. D 59, 077301 (1999).

[413] M. Apollonio et al., CHOOZ Coll., Phys. Lett. B 420, 397; C. Bemporad, Talk presented at the Ringberg Euroconference New Trends in Neutrino Physics, Ringberg Castle, Tegernsee, Germany, May, 1998.

[414] K. Zuber, Invited talk at the International Workshop on Simulations and Analysis Methods for Large Neutrino Detectors, Zeuthen, Germany, 6-9 Jul, 1998 (hep-ex/9810022).

[415] J.T. Peltoniemi, D. Tommasini and J.W.F. Valle, Phys. Lett. B 298, 383 (1993); J.T. Peltoniemi and J.W.F. Valle, Nucl. Phys. B 406, 409 (1993); D.O. Caldwell and R.N. Mohapatra, Phys. Rev. D 48, 3259 (1993); E. Ma and P. Roy, ibid 52, R4780 (1995); S. Goswami, ibid 55, 2931 (1997); A.Yu. Smirnov and M. Tanimoto, ibid 55, 1665 (1997); N. Gaur et al., ibid 58, 071301 (1998); E.J. Chun, C.W. Kim and U.W. Lee, ibid 58, 093003 (1998); V. Barger et al., ibid 58, 093016 (1998); K. Benakli and A.Yu. Smirnov, Phys. Rev. Lett. 79, 4314 (1997); E.J. Chun et al., Phys. Lett. B 357, 608 (1995); V. Barger, T.J. Weiler and K. Whisnant, ibid 427, 97 (1998); S.C. Gibbons et al., ibid 430, 296 (1998); J.J. Gomez-Cadenas and M.C. Gonzalez-Garcia, Z. Phys. C 71, 443 (1996); Q.Y. Liu and A.Yu. Smirnov, Nucl. Phys. 524, 505 (1998); S. Mohanty, D.P. Roy and U. Sarkar, Phys. Lett. B 445, 185 (1998).

[416] S.M. Bilenky, C. Giunti and W. Grimus, Eur. Phys. J. C 1, 247 (1998).

[417] N. Okada and O. Yasuda, Int. J. Mod. Phys. A 12, 3669 (1997).

[418] I.E. Stockdale et al., CCFR Coll., Phys. Rev. Lett. 52, 1384 (1984).

[419] S.M. Bilenky, C. Giunti and W. Grimus, Proc. of Neutrino '96, Helsinki, June 1996, edited by K. Enqvist et al., p. 174, World Scientific, Singapore, 1997 (hep-ph/9609343).

[420] S.M. Bilenky, C. Giunti and W. Grimus, Proc. of the $5^{\text {th }}$ Workshop on High Energy Physics Phenomenology, IUCAA, Pune, India, January, 12-26, 1998 (hep-ph/9805368).

[421] S.M. Bilenky, C. Giunti, C.W. Kim and S.T. Petcov, Phys. Rev. D 54, 4432 (1996). 
[422] G. Conforto, A. Marchionni, F. Martelli and F. Vetrano, Phys. Lett. B 427, 314 (1998).

[423] S.M. Bilenky, C. Giunti and W. Grimus, Phys. Rev. D 57, 1920 (1998).

[424] S.M. Bilenky, C. Giunti, W. Grimus and T. Schwetz, preprint hep-ph/9804421 (1998) [to be published in Astropart. Phys.].

[425] S. Sarkar, Rept. Prog. Phys. 59, 1493 (1996); D.N. Schramm and M.S. Turner, Rev. Mod. Phys. 70, 303 (1998); K.A. Olive, preprint astro-ph/9707212; Nucl. Phys. (Proc. Suppl.) 70, 521 (1999); A.D. Dolgov, preprint astro-ph/9807134.

[426] R. Foot, M.J. Thomson and R.R. Volkas, Phys. Rev. D 53, 5349 (1996); X. Shi, ibid. 54, 2753 (1996); R. Foot and R.R. Volkas, ibid. 55, 5147 (1997); R. Foot, preprint hep-ph/9809315.

[427] S.M. Bilenky, C. Giunti, C.W. Kim and M. Monteno, Phys. Rev. D 57, 6981 (1998).

[428] S.M. Bilenky, C. Giunti and W. Grimus, Proc. of Neutrino '98, Takayama, Japan, June, 1998 hep-ph/9809368).

[429] M. Günther et al., Phys. Rev. D 55, 54 (1997); Phys. Lett. B 407, 219 (1997).

[430] G. Pantis, F. Simkovic, J.D. Vergados and A. Faessler, Phys. Rev. C 53, 695 (1996); F. Simkovic, J. Schwieger, G. Pantis and A. Faessler, Found. Phys. 27, 1275 (1997).

[431] Talks presented by A. Morales, H. Ejiri, F. Piquemal, H.V. Klapdor-Kleingrothaus and O. Cremonesi at Neutrino '98 434].

[432] F. Wilczek, Talk presented at Neutrino '98 [434, 1998 (hep-ph/9809509).

[433] C. Ryan and S. Okubo, Il Nuovo Cim. Suppl. 2, 234 (1964).

[434] International Conference on Neutrino Physics and Astrophysics Neutrino '98, Takayama, Japan, June 1998; WWW page: http://www-sk.icrr.u-tokyo.ac.jp/nu98.

[435] NOMAD WWW page: http://nomadinfo.cern.ch/. 